\title{
Fundamentals of collisionless shocks for astrophysical application, 1. Non-relativistic shocks
}

\author{
R. A. Treumann
}

Received: 22 December 2008 / Published online: 16 September 2009

(C) Springer-Verlag 2009

\begin{abstract}
A comprehensive review is given of the theory and properties of nonrelativistic shocks in hot collisionless plasmas-in view of their possible application in astrophysics. Understanding non-relativistic collisionless shocks is an indispensable step towards a general account of collisionless astrophysical shocks of high Mach number and of their effects in dissipating flow-energy, in heating matter, in accelerating particles to high-presumably cosmic-ray-energies, and in generating detectable radiation from radio to X-rays. Non-relativistic shocks have Alfvénic Mach numbers $\mathscr{M}_{A} \ll \sqrt{m_{i} / m_{e}}\left(\omega_{p e} / \omega_{c e}\right)$, where $m_{i} / m_{e}$ is the ion-to-electron mass ratio, and $\omega_{p e}, \omega_{c e}$ are the electron plasma and cyclotron frequencies, respectively. Though high, the temperatures of such shocks are limited (in energy units) to $T<m_{e} c^{2}$. This means that particle creation is inhibited, classical theory is applicable, and reaction of radiation on the dynamics of the shock can be neglected. The majority of such shocks are supercritical, meaning that non-relativistic shocks are unable to self-consistently produce sufficient dissipation and, thus, to sustain a stationary shock transition. As a consequence, supercritical shocks act as efficient particle reflectors. All these shocks are microscopically thin, with shock-transition width of the order of the ion inertial
\end{abstract}

R. A. Treumann: Visiting the International Space Science Institute, Bern, Switzerland.

\section{R. A. Treumann $(\varangle)$}

Department of Geophysics and Environmental Sciences, Geophysics Section, Ludwig-Maximilians-University Munich, Theresienstr. 37-41, 80333 Munich, Germany e-mail: rudolf.treumann@geophysik.uni-muenchen.de

\section{R. A. Treumann}

Department of Physics and Astronomy, Dartmouth College, Hanover, NH 03755, USA

Present Address:

R. A. Treumann

International Space Science Institute, Hallerstrasse 6, 3012 Bern, Switzerland 
length $\lambda_{i}=c / \omega_{p i}$ (with $\omega_{p i}$ the ion plasma frequency). The full theory of such shocks is developed, and the different possible types of shocks are defined. Since all collisionless shocks are magnetised, the most important distinction is between quasiperpendicular and quasi-parallel shocks. The former propagate about perpendicularly, the latter roughly parallel to the upstream magnetic field. Their manifestly different behaviours are described in detail. In particular, although both types of shocks are non-stationary, they have completely different reformation cycles. From numerical full-particle simulations it becomes evident that, on ion-inertial scales close to the shock transition, all quasi-parallel collisionless supercritical shocks are locally quasiperpendicular. This property is of vital importance for the particle dynamics near the quasi-parallel shock front. Considerable interest focusses on particle acceleration and the generation of radiation. Radiation from non-relativistic shocks results mainly in wave-wave interactions among various plasma waves. Non-thermal charged particles can be further accelerated to high energies by a Fermi-like mechanism. The important question is whether the shock can pre-accelerate shock-reflected particles to sufficiently high energies in order to create the seed-population of the non-thermal particles required by the Fermi mechanism. Based on preliminary full-particle numerical simulations, this question is answered affirmatively. Such simulations provide ample evidence that collisionless shocks with high-Mach numbers-even when non-relativistic — could probably by themselves produce the energetic seed-particle population for the Fermi-process.

Keywords Collisionless shocks $\cdot$ Supercritical shocks $\cdot$ Shock kinetics · Shock reformation $\cdot$ Shock acceleration

\section{Introduction}

Shocks are abundant in the Universe. They are generated when cosmic obstacles are exposed to fast (supermagnetosonic) flows or, vice versa, when objects such as comets, planets, galaxies and so on pass across their gaseous environments at high speeds. They also form when fast flows interact in head-on collisions, overturn each other or are stopped by their resting environments. From an observational point of view, however, astrophysics depends solely on the detection of emitted radiation (from radio waves to cosmic rays) and does not permit measurements to be performed in situ on any of those objects with-possibly-the exclusion of shocks in the solar system and the heliosphere. Because of this restriction, only those shocks can be observed and identified from Earth — or with the support of spacecraft from Earth's orbit—which radiate in one of the observable bands of the entire electromagnetic spectrum.

In order to radiate, shocks must be ionised, ${ }^{1}$ and in most cases they also involve magnetic fields. Astrophysical shocks emerge, indeed, from highly if not fully ionised

\footnotetext{
1 Neutral atoms do also radiate when excited by either binary collisions or absorption of radiation. In treating collisionless shocks, the first is excluded from consideration by the collisionless property (i.e. the required high neutral densities for collisions to become important), the second requires the presence of external illumination. In strength, the electromagnetic interaction by far exceeds the interaction with any neutral-atom population that may be present in collisionless shocks. Even when illuminated and radiating,
} 
magnetised plasmas. These observable shocks form a subclass of all shocks that can evolve, the subclass of strong shocks, in the sense that the shock generates radiation of intensity sufficiently strong that it can be detected and from which information can be inferred about the state of matter in its sources. Quite naturally, the astrophysical interest in shocks is determined by this capability of shocks to either radiate or accelerate particles to energies sufficiently high that their various effects can either be seen in radiation or can reach the solar system as part of the measured energetic cosmic ray flux.

The subclass of strong shocks further divides into nonrelativistic and relativistic shocks. In a follow-up review we will be dealing with relativistic shocks which are weak enough to not generate elementary particles, in other words shocks which do not force us to refer to elementary particle physics and field theory. In contrast, the present review treats the group of nonrelativistic shocks which evolve in flows of velocity $V \ll c$ much less than the velocity of light. Their physics is fundamental for the understanding of shocks in general while still being relatively simple. A weaker condition, which coincides with the exclusion of relativistic shocks, is that we consider only shocks which do not radiate so strongly that the reaction of the radiation must be taken into account in shock dynamics. This excludes shocks generated in the vicinity of massive objects such as neutron stars and black holes, jets ejected from massive objects, and the highly relativistic outflows that surround them. In terms of the Alfvénic ${ }^{2}$ Mach number $\mathscr{M}_{A}=V / V_{A}$ the non-relativistic assumption holds for

$$
\mathscr{M}_{A} \ll\left(\frac{m_{i}}{m_{e}}\right)^{\frac{1}{2}} \frac{\omega_{p e}}{\omega_{c e}} \approx 1.4 \times 10^{4} \frac{\sqrt{N_{e,\left[\mathrm{~cm}^{-3}\right]}}}{B_{[\mathrm{nT}]}}, \quad T \ll m_{e} c^{2} \approx 0.511 \mathrm{MeV}
$$

with kinetic temperature $T$ in energy units, ${ }^{3}$ electron density $N_{e}$ in $\mathrm{cm}^{-3}$, and magnetic field $B$ in nT, $m_{i}, m_{e}$ are the respective ion and electron masses. $\omega_{p e}=e \sqrt{N_{e} / \epsilon_{0} m_{e}}$ is the electron plasma frequency in a plasma of density $N_{e}$, and $\omega_{c e}=e B / m_{e}$ is the electron cyclotron frequency in the magnetic field $B$. Finally, $V_{A}=B / \sqrt{\mu_{0} N_{e} m_{i}}$ is the Alfvén velocity. The condition on the Mach number $\mathscr{M}_{A}$ is supplemented by the

\footnotetext{
Footnote 1 continued

the radiation from the neutral atoms has no effect on collisionless shock dynamics. This does not preclude the optical emission of the neutral component from being of interest in the identification of shock properties (temperature, density etc.) remotely.

2 The Mach number is usually defined with respect to the magnetosonic velocity as $\mathscr{M}=V / c_{m s}$ with $c_{m s}^{2}=V_{A}^{2}+c_{s}^{2}$ (see the text farther below), where $c_{s}$ is the sonic speed. In the overwhelming number of cases, collisionless plasmas are magnetised with the magnetic field dominating the dynamics. Therefore the important velocity is the Alfvén speed $V_{A} \gtrsim c_{S}$, such that the Mach number practically reduces to the Alfvénic Mach number, with the sound speed causing a small correction only. When the sound speed dominates, the shock becomes either purely electrostatic or even ordinary gas-dynamic. The latter case implies the presence of collisions and is not considered here.

3 Throughout this paper we use SI units. Then, conventionally, $T(\mathrm{~K})$ would be measured in degrees $\mathrm{K}$. In plasma and shock theory it is, however, more practical to measure it in energy units $T=k_{B} T(\mathrm{~K})$, with $k_{B}$ the Boltzmann constant, for instance in electron Volts eV $\left(1 \mathrm{eV}=1.602 \times 10^{-19} \mathrm{~J}\right.$ corresponds to a temperature of $T(\mathrm{~K})=1.16 \times 10^{4} \mathrm{~K}$ ). Number densities $N$ will generally be given in $\mathrm{m}^{-3}$ (except when explicitly indicated as here, where they are in $\mathrm{cm}^{-3}$ ). Moreover, in spite of their exceptional importance in collisionless shocks, magnetic fields $B$ are weak; in all nonrelativistic shock applications it is most practical to measure them in Nanoteslas $\left(\operatorname{lnT}=10^{-9} \mathrm{~T}=10^{-5} \mathrm{G}\right)$.
} 
condition on the temperature $T$ in order to prevent particle creation. It allows for high Mach numbers. In strong magnetic fields and very dilute plasmas the nonrelativistic approximation breaks down. Restricting to collisionless shocks further implies that we exclude objects which are so dense that shock formation proceeds on time scales longer than the binary collisional time scale $\tau_{\text {coll }} \simeq \lambda$ coll $/ v_{e}$, where $T_{e}=m v_{e}^{2} / 2$ is the (nonrelativistic) electron temperature (in energy units), and $v_{e}$ is the thermal velocity of the electrons. Expressed differently, we exclude all shocks whose width $\Delta_{\text {sh }}$ is comparable to or larger than the collisional mean free path $\lambda_{\text {coll }}=\left(\sigma_{\text {coll }} N\right)^{-1}$, with density $N$, and $\sigma_{\text {coll }} \simeq 5 \times 10^{-19} \mathrm{~m}^{-2}$ the binary collisional cross section. The vast majority of nonrelativistic astrophysical shocks evolve under conditions of highly dilute matter with $N \lesssim 10^{6} \mathrm{~m}^{-3}$, such that $\lambda$ coll $\gg \Delta_{\text {sh }}$ grossly exceeds the shock width. For them, the first of the above conditions holds as long as $B<10^{3} \mathrm{nT}$. Such shocks are collisionless, and the question of how they are generated poses a nontrivial problem.

The paradigm of nonrelativistic collisionless shocks are the shocks in the heliosphere. These are solar coronal shocks, which are generated in the interaction of solar outbursts on all scales, interplanetary shocks which accompany coronal mass ejections (CMEs) and corotating interaction regions in the solar wind, the various planetary and cometary bow shocks, and the solar wind termination shock that is generated at the outer boundary of the heliosphere where the solar wind is stopped by the interaction with the interstellar medium. These shocks are the only ones in the entire Universe which can be accessed in situ. For illustration we will frequently refer to one or the other of them in the following.

Most of those shocks do, in fact, emit radio radiation at the local plasma frequency $\omega \sim \omega_{p e}=2 \pi f_{p e}$, with $f_{p e} \approx 8.979 \sqrt{N_{e}} \mathrm{kHz}$ (with electron density $N_{e}$ in $\mathrm{cm}^{-3}$ ), and its harmonic $\omega \sim 2 \omega_{p e}$. Energetically this radiation is negligible. Moreover, with the heliosphere being a dilute cavity of (quasi-neutral) density $N_{e}=N_{i}=N \ll$ $N_{\text {ISM }}$, blown into the surrounding interstellar medium of density $N_{\text {ISM }}$ by the supermagnetosonic solar wind, the frequency of the radio emission from the various types of shocks in the heliosphere (except those near the sun) is below the plasma frequency cut-off $f_{p e}^{\mathrm{ISM}}$ in the interstellar medium and is thus confined to the heliosphere. ${ }^{4}$ In other words, looked at from the outside, the heliosphere is apparently 'shockfree'. From here we conclude that a large fraction of the collisionless nonrelativistic shocks in the Universe are dark. Since the heliosphere is full of standing and travelling shocks, which contribute substantially to particle acceleration, plasma heating, generation of turbulence and energy dissipation, we also conclude that such 'dark shocks' in the Universe are abundant, playing an important role in the dynamics of the collisionless matter in many but the most compact astrophysical objects (neutron stars, magnetars, black holes and AGNs). This fact alone justifies the separate consideration of the formation, properties and effects of this class of invisible subjects.

Those shocks that are not confined to stellar wind bubbles like the heliosphere become visible in some part of the electromagnetic spectrum. Their visibility depends

\footnotetext{
4 These shocks are non-relativistic, so the emitted radiation is neither boosted nor beamed. Even the radiation from the shocks in the inner heliosphere close to the Sun with frequency high enough for escape, decays in intensity about as the inverse square of the distance and is thus barely detectable remotely.
} 
on the radiation mechanism and the emission measure of the shock, i.e. the plasma density $N$ and the width $\Delta_{\text {sh }}$ of the shock ramp in order to generate sufficiently intense radiation. Generation of radiation requires the acceleration of electrons to high, possibly relativistic energies even in nonrelativistic shocks. Shocks of this kind are known from supernova remnants (SNRs), and from stellar and galactic bow shocks. Moreover, the termination shocks from stellar winds and the termination shocks generated when plasma jets ejected from massive objects interact with the interstellar or galactic environments to finally form giant lobes and resolve into filaments which fill the lobes providing it with a texture of mutually interacting shocks. These shocks are known as the 'external' shocks, some of them being relativistic.

In the present article we review the theory and numerical simulations of the three different types of nonrelativistic collisionless shocks, subcritical shocks, supercritical quasi-perpendicular shocks and supercritical quasi-parallel shocks, and compare them with in situ shock observations in the heliosphere as well as with available remote observations of some of the known astrophysical shocks. Presumably, subcritical shocks are of lesser interest in application to astrophysical objects. They barely radiate and are thus manifestly black, forming a class that can in a wider sense be attributed to turbulence. Historically, these were the shocks that were thought of as the genuine shocks, and it took about two decades until it was recognised that the majority of shocks which are of interest in space and astrophysics are supercritical.

Nonrelativistic collisionless shocks in highly dilute ordinary matter of the kind we encounter in interplanetary space, in the heliosphere, stellar environments, galaxies and clusters of galaxies are mediated by medium and long range interactions between charged particles introduced by the electromagnetic fields $(\mathbf{E}, \mathbf{B})$ that act on the free charges via the Lorentz force

$$
\mathbf{F}_{L}=q_{ \pm}(\mathbf{E}+\mathbf{v} \times \mathbf{B})
$$

In order to produce the free charges $q_{ \pm}= \pm e$ (with $e$ the elementary charge) the matter must be hot, of temperature $T>\mathscr{E}$ ion larger than the ionisation energy which is of the order of $\mathscr{E}_{\text {ion }} \sim$ several $10 \mathrm{eV}$ (or $>10^{5} \mathrm{~K}$ ). Collisionless shocks are plasma shocks. They are subject to the different correlated dynamics of ions and electrons. The difference of three orders of magnitude in the masses $m_{ \pm}$of these two constituents brings with it a severe complication and an enormous richness of effects, the current knowledge of which we will describe in greater detail. However, there is no way of treating collisionless shocks analytically. Most knowledge is based on numerical simulations in one and two dimensions. In general, shocks are three-dimensional objects. The Crab nebula, for instance, shows this impressively. Three-dimensional simulations which account for the full dynamics of both electrons and ions are still out of reach, however.

This is a substantial hurdle in the completion of collisionless shock theory, in particular that which concerns simulations of large three-dimensional global shocks, for realistic ion-to-electron mass ratios and with large particle numbers. It is unknown how representative the available simulations are for constructing a final collisionless shock theory. Experience shows that every five to ten years a further step ahead is made that forces one to abandon earlier conclusions and results. Such was the case in the 
early seventies when magnetohydrodynamic shock theory turned out to be too simple an approximation which, however, was not abandoned for another decade. Then in the eighties hybrid theories with few particles came into fashion providing interesting results about shock reformation as the last word. This ultimately had to be given up when, in the nineties, full Particle-In-Cell (PIC) simulations took over and showed that the entire reformation theory worked completely differently for quasi-perpendicular and quasi-parallel shocks. The recent rise of full particle simulations using real electron-to-ion mass ratios promises further fundamental changes in shock theory as the interaction between electrons and ions and the different instabilities in which both particles are involved will presumably cause another revolution-in particular in the highly nonlinear, non-homogeneous shock state. In application to astrophysical shocks it must be kept in mind that all high-resolution simulations are restricted to small systems. They just provide local information on the physics of shocks. Anticipating a complete theory requires embedding the small scale simulations into large-scale systems. Such approaches are still completely illusionary from a practical point of view, because the fluid and the microscopic approaches have technically little in common.

Another key question concerns the interaction between the shock and the ambient particle population. This question is fundamental in astrophysical applications. We will briefly treat it in a separate section below. The problem of particle acceleration by shocks has been with us for more than half a century already, being resolved only partially. Here, we will not treat the full problem of acceleration. There are plenty of reviews available in which shock acceleration is the focus. Rather we will, at least to some extent, illuminate the role of collisionless shocks in providing the necessary seed particle population for injection into the general Fermi-like acceleration process.

\subsection{Observational background}

The only safe observations of collisionless shocks in space come from the multitude of spacecraft crossings of planetary and interplanetary shocks in the Earth's heliospheric environment, i.e. in the only stellar wind that is accessible to observation in situ: the solar wind. This review will, just for this reason, mainly refer to these well established and indisputable observational facts. For the intentional centre of this review, shocks in astrophysics, an enormous amount of observational material is available on radiation from astrophysical systems that show filamentary signatures. Yet no unambiguous information about the nature and properties of the presumable shocks that are suspected to relate to these structures is available. The interpretation of this radiation uses the well founded assumption that shocks are involved. However, there is a large amount of speculation around the nature and physical properties of many of these shocks. While being well founded in themselves, such speculations have little physical support from the known physics of collisionless shocks, of which the only tested data come from space physics. Experience with the accessible interplanetary medium has shown that the availability of a single measurement in situ can completely changed our views of the interplanetary space and matter. Probably one single in situ measurement in the astrophysical environment would change our views in a similar way. So far, astrophysics has extensively borrowed from space physics in many respects, and it is 
probably the safest way to stay as close as possible to reality by carefully extrapolating from interplanetary to more remote astrophysical systems.

The objects where shocks are expected to exist in astrophysics spread from stellar environments to the extragalactic matter in clusters of galaxies. Clearly this range covers an enormous variety of different parameters, plasma densities, temperatures, Mach numbers and sometimes compositions. Stellar environments include the stellar coronae and different kinds of stellar winds (cf. Kudritzki and Puls 2000). As is known from the observation of the solar corona, the continuous outflow of the solar wind as well as the ejection of solar material during violent solar events, such as solar flares and CMEs, cause blast shock waves. These propagate radially outward on the background of the expanding magnetised solar wind while corotating with the sun and interacting with the ecliptic current sheet and solar/interplanetary magnetic sector structure. In this interaction they may form both forward and reverse shocks, interact with the heavenly bodies and their bow shocks, and ultimately interact with the interstellar gas at the outskirts of the heliosphere. Similar kinds of shocks are expected to exist in all hot stellar environments. Observation of flares from other magnetised stars allows us to extend these arguments to other stars, in particular to active stars with much stronger stellar winds than the sun: the hot massive stars, flare stars, Wolf-Rayet stars, etc. Like the solar wind, their stellar winds will blow holes into the interstellar gas, creating 'asterospheres'. These will give rise to huge and possibly strong shocks where they interact with the interstellar matter, again in similarity to the heliospheric termination shock. It is widely believed that these extended and strong stellar termination shocks are one of the main actors in accelerating galactic cosmic rays (see, e.g. Blandford and Eichler 1987), even though the very recent observations of the heliospheric termination shock are ambiguous in proving this assertion. Since the energy stored in these shocks and possibly transferred to cosmic rays comes from the magnetised stellar wind flow, a simple rule-of-thumb estimate of the available energy can be based on the electric potential drop across the 'asterosphere'. In the heliosphere, with the termination shock located at a distance of roughly $100 \mathrm{AU}$ and a convection electric field of $\sim 5 \mathrm{mV} / \mathrm{m}$, the available total energy is of the order of $\mathscr{E} \sim 2 \times 10^{12} \mathrm{eV}$. Much of it is dissipated in the interaction with interstellar gas. However, the remaining fraction is high enough for accelerating particles to cosmic ray energies of several $100 \mathrm{MeV} /$ nucleon.

The astrophysical paradigm of strong, mildly relativistic shocks are the shocks in the outer shells of SNRs such as the Crab nebula. Excluding its massive inner engine, which is known to be a rotating neutron star which radiates in X-rays and drives a highly relativistic wind, we are left with the shell which expands at velocity of 1,500 $\mathrm{km} / \mathrm{s}$, interacts with the interstellar gas and has not yet reached dynamic equilibrium. Its highly filamentary structure suggests that various external shocks are generated in this interaction on various scales with complicated surface structures and directions of propagation. In addition, internal shocks produced in the supernova outflow when it collides with the retarded, mass loaded or reflected flow, are also present. SNR shocks, such as the web of Crab shocks, are strong candidates for generating highenergy cosmic rays. Galaxies contain many of these SNRs, which may be responsible for part of the galactic cosmic-ray spectrum. Other galactic shocks are related to the galactic spirals and to internal galactic interactions. In addition, galaxies themselves emit galactic winds that interact with the intergalactic medium, producing galactic 
termination shocks which are seen in radio emissions. Their typical speed is $\sim 500$ $\mathrm{km} / \mathrm{s}$ comparable to the speed of the solar wind. They may reach out from the galaxy to distances of $\sim 100 \mathrm{kpc}$ or more.

In clusters of galaxies, the medium is denser than around field galaxies. Mass densities and temperatures are in the intervals $10^{-29}<N_{m}<10^{-27} \mathrm{~kg} / \mathrm{m}^{3}$ and $0.1<T<30$ $\mathrm{keV}$, respectively. The shocks may thus reach temperatures of the order of several $\mathrm{keV}$, or even higher than $10 \mathrm{keV}$, which can be observed in thermal x-ray bremsstrahlung and synchrotron radio emission since the matter is weakly magnetised. In rich clusters in approximate virial equilibrium containing hundreds to thousands of galaxies, the galaxies themselves move at relative velocities of several $1,000 \mathrm{~km} / \mathrm{s}$ across the intergalactic medium. This intracluster matter is hot and magnetised. Fast enough galaxies, when moving against it, may generate bow shocks and have extended wakes. In rich clusters one may thus expect that some of the turbulence inside the intracluster matter is due to the presence of these galactic bow shocks and galactic wakes. Such galactic bow shocks are huge: they can have diameters of the order of several 10-100 kpc. These shocks are magnetic and strong and have kinetic properties. As long as they are non-relativistic they should be subject to application of the theory which will be reviewed in this paper. Shocks are also generated when galaxies collide with each other. The probability of this happening is highest in rich clusters. In this case the shocks might become very strong, indeed.

\subsection{Rankine-Hugoniot conditions}

Shocks propagate on the plasma background with group velocity of the wave out of which they emerge. Shocks can be classified from various points of view. The simplest classification is with respect to their electrodynamic properties.

\subsubsection{When are shocks collisionless?}

Collisionless high-temperature dilute and fully ionised plasmas take advantage of nonlocal, non-binary, 'anomalous collisions' between the particles and the existing self-consistently generated electromagnetic fields. These 'anomalous collisions' are in fact long-range collective interactions between groups of particles and fields leading to correlations that, in turn, cause irreversible dissipation processes resulting in heating and generation of entropy. These collective processes allow the plasma to return to a thermodynamic state.

Collisionless shock waves form when a large obstacle is put into a plasma flow that in the frame of the obstacle is super-Alfvénic (or super-magnetosonic). Super-Alfvénic flows have Mach numbers $\mathscr{M}_{A}=V / V_{A}>1$, where $\mathbf{V}_{A}=\mathbf{B} / \sqrt{\mu_{0} m_{i} N}$ is the Alfvén velocity in the magnetized plasma of density $N$ and magnetic field $\mathbf{B}$, and $m_{i}$ is the ion mass. ${ }^{5}$ Super-magnetosonic flows have Mach numbers $\mathscr{M} \equiv \mathscr{M}_{m s}=V / c_{m s}>1$, where $c_{m s}^{2}=V_{A}^{2}+c_{s}^{2}$ is the square of the magnetosonic speed, and $c_{s}^{2}=\partial \mathrm{P} / \partial \rho$ is the

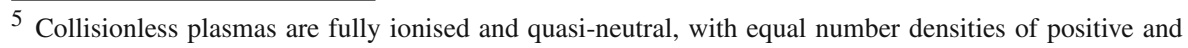
negative charges $N_{e}=N_{i}=N$.
} 
square of the sound speed, with $\mathrm{P}$ the isotropic pressure, and $\rho=m_{i}\left(1+m_{e} / m_{i}\right) N \approx$ $m_{i} N$ the mass density. In a plasma with temperatures $T_{e}, T_{i}$ (in energy units) and adiabatic indices $\gamma_{e, i}$ for electrons and ions, respectively, we have $c_{s}^{2}=\left(\gamma_{e} T_{e}+\gamma_{i} T_{i}\right) / m_{i}$. The magnetosonic speed depends on the wave propagation angle $\theta$ with respect to the magnetic field:

$$
c_{m s}^{2}(\theta)=c_{m s}^{2} \pm\left[\left(V_{A}^{2}-c_{s}^{2}\right)^{2}+4 V_{A}^{2} c_{s}^{2} \sin ^{2} \theta\right]^{\frac{1}{2}}
$$

where $c_{m s}$ is the above (angle-independent) expression. It splits into two branches, $c_{m s}^{+}$and $c_{m s}^{-}$, of which only the fast mode propagates perpendicular to the magnetic field. In all other directions all three plasma modes, the two magnetosonic and the Alfvén wave, can exist. Each can evolve into a shock if the flow speed exceeds its group velocity. Hence, in a plasma three types of shock waves can, in principle, exist: the fast, slow, and Alfvénic (or intermediate) shocks, respectively.

The requirement on the obstacle for a shock to evolve is that its diameter $D$ in the two directions perpendicular to the flow must be large compared to the intrinsic scales of the flow while at the same time being much less than $\lambda_{\text {coll }}$. The largest intrinsic scale is the ion gyro- radius $r_{c i}=V_{i \perp} / \omega_{c i}$, with $V_{i \perp}$ the ion velocity perpendicular to $\mathbf{B}$, and $\omega_{c i}=e B / m_{i}$ the ion cyclotron frequency. With the Coulomb mean free path $\lambda_{\mathrm{C}}=\left(N \sigma_{\mathrm{C}}\right)^{-1}$, where $\sigma_{\mathrm{C}}=\left(16 \pi N^{2} \lambda_{D}^{4}\right)^{-1}$ is the Coulomb cross section, one thus has

$$
r_{c i} \ll D \ll \lambda_{\mathrm{C}}
$$

( $\lambda_{D}=v_{e} / \omega_{p e}$ is the Debye length, and $v_{e}$ is the electron thermal velocity). The shock forms an extended surface $F_{S}(\mathbf{r})=r$ that is bent around the obstacle, where $r$ is the radius vector (for instance measured from the centre of the obstacle) to one point on the shock surface. The radius of curvature $R_{c} \gg \Delta$ of the shock perpendicular to the flow will always be much larger than the width $\Delta \sim r_{c i}$ of the shock in the direction of flow, the latter being of the order of a few ion gyro- radii only. Collisionless shocks can thus, in good approximation, be considered as thin, locally flat surfaces of width $\Delta$ and outer shock-normal unit vector

$$
\mathbf{n}(\mathbf{r})=-\frac{\nabla F_{S}(\mathbf{r})}{\left|\nabla F_{S}(\mathbf{r})\right|}, \quad \nabla \simeq-\mathbf{n} \frac{\partial}{\partial n} \sim-\mathbf{n} \frac{[\cdots]}{\Delta}
$$

Locally, of all spatial derivatives $\partial / \partial x \sim \partial / \partial y \ll \partial / \partial n$ only the derivative across the shock front counts. The gradient operator $\nabla$ reduces to the derivative in the direction opposite to $\mathbf{n}$ with coordinate $n$, where the brackets $[\cdots]=(\cdots)_{2}-(\cdots)_{1}$ stand for the difference between the values of the quantity under consideration downstream in Region 2 behind the shock and the value upstream in Region 1 in front of the shock. ${ }^{6}$

\footnotetext{
6 In plasma particle simulations (in particular in more than one dimension), where the shock-to a certain degree trivially - becomes nonstationary, the local shape and width of the shock vary in time. Then the shock normal is defined instantaneously and locally and is no longer a constant unit vector.
} 


\subsubsection{Basic equations}

Collisionless shock waves represent the final result of collective interactions in a plasma that is streaming at high velocity relative to some large-scale obstacle. In this interaction very many charged particles and, in addition, the full electromagnetic fields are involved. It is thus reasonable that they cannot be described by test particle theory which considers the motion of non-interacting particles. Test particle theory can, however, be applied if one is not interested in the formation of shocks but instead in their effect on small numbers of particles.

The basic equations on which shock physics is founded are the kinetic equations of a plasma (cf. e.g., Tidman and Krall 1971, and others) or, at best, some of its simplifications, in addition to the full set of the equations of electrodynamics. These equations reduce to the (non-relativistic) Vlasov-Maxwell set of equations

$$
\frac{\partial F^{ \pm}}{\partial t}+\mathbf{v} \cdot \nabla F^{ \pm}+\frac{e_{ \pm}}{m_{ \pm}}(\mathbf{E}+\mathbf{v} \times \mathbf{B}) \cdot \nabla_{\mathbf{v}} F^{ \pm}=0
$$

where $F^{ \pm}(\mathbf{v}, \mathbf{x}, t)$ are the electron and ion phase space distributions, distinguished by the respective + and - signs, which depend on the six-dimensional phase space velocity, $\mathbf{v}$, and real space, $\mathbf{x}$, coordinates. $m_{+} \equiv m_{i}$ and $m_{-} \equiv m_{e}$ are the ion and electron masses, respectively; $e_{+}=e$ is the ion charge, $e_{-}=-e$ electron charge, $e$ the elementary charge, and $\mathbf{E}(\mathbf{x}, t), \mathbf{B}(\mathbf{x}, t)$ are the electromagnetic fields which are independent of velocity while being functions of space and time. Finally, $\nabla_{\mathbf{v}} \equiv \partial / \partial \mathbf{v}$ is the velocity gradient operator acting on the phase space distributions. The two Vlasov equations (6) are mutually coupled via the self-consistent electromagnetic fields through Maxwell's equations

$$
\left.\begin{array}{ll}
\nabla \times \mathbf{B}=\mu_{0} \epsilon_{0}(\partial \mathbf{E} / \partial t)+\mu_{0} \sum_{ \pm} e_{ \pm} \int \mathrm{d} v^{3} F^{ \pm} \mathbf{v}, & \nabla \cdot \mathbf{B}=0 \\
\nabla \times \mathbf{E}=-(\partial \mathbf{B} / \partial t), & \nabla \cdot \mathbf{E}=\epsilon_{0}^{-1} \sum_{ \pm} e_{ \pm} \int \mathrm{d} v^{3} F^{ \pm}
\end{array}\right\}
$$

The coupling between fields and particles is provided by the electric current and particle densities, i.e. by the first and zero-order moments of the phase space distributions $F^{ \pm}$that appear on the right-hand sides of Ampère's and Poisson's laws in Eq. 7.

Shocks evolve from infinitesimal wave disturbances. One hence considers two different states of the plasma: the final slowly evolving state of the fully developed shock, and the time-dependent evolution of an infinitesimal disturbance. The latter starts from the thermal fluctuation level in the plasma and grows until it forms the shock. In the first state the shock possesses a distinct shock profile while in the second state one deals with initially infinitesimal fluctuations. When the fluctuation amplitude approaches the shock strength the two different ways of looking at the shock should ideally lead to the same result. According to this distinction one divides all field and plasma quantities, 
$A$, into their slowly varying averages, $\langle A\rangle$, and fast fluctuations, $\delta A$, superimposed on the averages according to the prescription ${ }^{7}$

$$
A=\langle A\rangle+\delta A, \quad\langle\delta A\rangle=0
$$

\subsubsection{Averages and fluctuations}

Let us indicate the averages $\langle\cdots\rangle$ of the distribution functions and fields by the subscript 0 , and the fluctuations by small letters $f, \mathbf{e}, \mathbf{b}$. Then

$$
\frac{\partial F_{0}^{ \pm}}{\partial t}+\mathbf{v} \cdot \nabla F_{0}^{ \pm}+\frac{e_{ \pm}}{m_{ \pm}}\left(\mathbf{E}_{0}+\mathbf{v} \times \mathbf{B}_{0}\right) \cdot \nabla_{\mathbf{v}} F_{0}^{ \pm}=-\frac{e_{ \pm}}{m_{ \pm}}\left\langle(\mathbf{e}+\mathbf{v} \times \mathbf{b}) \cdot \nabla_{\mathbf{v}} f^{ \pm}\right\rangle
$$

is the kinetic equation for $F_{0}^{ \pm}(\mathbf{v}, \mathbf{x}, t)$ with $F_{0}$ varying on much longer spatial and temporal scales than the fluctuations such that $\langle f, \mathbf{e}, \mathbf{b}\rangle=0$. The pseudo-collision term on the right accounts for the correlations between fluctuations and particles. In contrast to the Vlasov equation, the Maxwell equations (7) retain their form with the sole difference that the full distribution functions $F^{ \pm}$appearing in the electric current density in Ampère's law and the space charge term in Poisson's equation are to be replaced by their average counterparts $F_{0}^{ \pm}$, yielding

$$
\left.\begin{array}{rlrl}
\nabla \times \mathbf{B}_{0}=\mu_{0} \epsilon_{0}\left(\partial \mathbf{E}_{0} / \partial t\right)+\mu_{0} \sum_{ \pm} e_{ \pm} \int \mathrm{d} v^{3} F_{0}^{ \pm} \mathbf{v}, \nabla \cdot \mathbf{B}_{0} & =0 \\
\nabla \times \mathbf{E}_{0}=-\left(\partial \mathbf{B}_{0} / \partial t\right), & \nabla \cdot \mathbf{E}_{0}=\epsilon_{0}^{-1} \sum_{ \pm} e_{ \pm} \int \mathrm{d} v^{3} F_{0}^{ \pm}
\end{array}\right\}
$$

Subtracting the averaged equations, the evolution of the fluctuating quantities $f=$ $F-F_{0}, \mathbf{e}=\mathbf{E}-\mathbf{E}_{0}, \mathbf{b}=\mathbf{B}-\mathbf{B}_{\mathbf{0}}$ is described by

$$
\begin{aligned}
\frac{\partial f^{ \pm}}{\partial t} & +\mathbf{v} \cdot \nabla f^{ \pm}+\frac{e_{ \pm}}{m_{ \pm}}\left(\mathbf{E}_{0}+\mathbf{v} \times \mathbf{B}_{0}\right) \cdot \nabla_{\mathbf{v}} f^{ \pm}=-\frac{e_{ \pm}}{m_{ \pm}}(\mathbf{e}+\mathbf{v} \times \mathbf{b}) \cdot \nabla_{\mathbf{v}} F_{0}^{ \pm} \\
& -\frac{e_{ \pm}}{m_{ \pm}}(\mathbf{e}+\mathbf{v} \times \mathbf{b}) \cdot \nabla_{\mathbf{v}} f^{ \pm}+\frac{e_{ \pm}}{m_{ \pm}}\left\langle(\mathbf{e}+\mathbf{v} \times \mathbf{b}) \cdot \nabla_{\mathbf{v}} f^{ \pm}\right\rangle
\end{aligned}
$$

which is the equation for $F_{0}$. It must be complemented by the Maxwell-equations for e, b which are of the usual form (7) with $F^{ \pm}$replaced by $f^{ \pm}$.

Clearly, in order for the shock to evolve from fluctuations, the width $\Delta_{\text {sh }}$ of the shock transition is larger than the wavelengths of the fluctuations. The three "collision terms" on the right are responsible for anomalous dissipation and are thus the most interesting terms in any theory that deals with the evolution of shock waves. They

\footnotetext{
7 The average $\langle\cdots\rangle$ is understood as an ensemble average, which can be reduced to averages over spatial and temporal scales (becoming sums or integrals). The scales are arbitrary, but are assumed to be larger than the scales of the fluctuations.
} 
prevent large amplitude waves from indefinite steeping and provide the required dissipation of kinetic energy and entropy generation that may lead to shock formation and stabilisation. In its general version the above equations should contain the whole physics of the shock including all collective processes which occur in the collisionless state before binary particle collisions come into play.

\subsubsection{Conservation laws}

The global plasma and field quantities, density $\langle N\rangle=N_{0}$, flow velocity $\langle\mathbf{V}\rangle=\mathbf{V}_{0}$ respectively momentum density $\langle N \mathbf{V}\rangle=N_{0} \mathbf{V}_{0}$, pressure $\langle\mathrm{P}\rangle=\mathrm{P}_{0}$, magnetic field $\langle\mathbf{B}\rangle=\mathbf{B}_{0}$, electric field $\langle\mathbf{E}\rangle=\mathbf{E}_{0}$, current density $\langle\mathbf{j}\rangle=\mathbf{j}_{0}$, entropy $\mathscr{S}$ and so on are defined as average moments of the global distribution function $\langle F\rangle=F_{0}$ according to the prescription

$$
M^{i}=\int \mathrm{d} v^{3} \mathbf{v}^{i} F
$$

for taking the moment of order $i$. Here $\mathbf{v}^{i}=\mathbf{v} \ldots \mathbf{v}$ is understood as the $i$-fold dyadic product. The first three moments are

$$
N=\int \mathrm{d} v^{3} F, \quad N \mathbf{V}=\int \mathrm{d} v^{3} \mathbf{v} F, \quad \mathbf{P}=m \int \mathrm{d} v^{3}(\mathbf{v}-\mathbf{V})(\mathbf{v}-\mathbf{V}) F
$$

The diagonal of the pressure tensor $\mathrm{P}$ gives the average energy density; it defines the local temperatures $T_{\|}, T_{\perp}$ parallel and perpendicular to the average magnetic field. Operating with these definitions on the average Vlasov equation (9) produces the well-known full-i.e. infinite — set of magnetogasdynamic equations for the infinite chain of moments of $F^{ \pm}$for each particle species $\pm=e, i$. The first two of them are

$$
\begin{gathered}
\frac{\partial N^{ \pm}}{\partial t}+\nabla \cdot(N \mathbf{V})^{ \pm}=0 \\
\frac{\partial(N \mathbf{V})^{ \pm}}{\partial t}+\nabla \cdot(N \mathbf{V V})^{ \pm}+\frac{1}{m_{ \pm}} \nabla \cdot \mathbf{P}^{ \pm}
\end{gathered}
$$

where $\mathscr{C}$ is the pseudo-collision term. This term conserves particle number (or mass). Wave particle interactions neither change particle number nor mass density. In the first order moment equation a wave friction term appears that has the explicit form

$$
-\frac{1}{m_{ \pm}}\left\{\frac{1}{\mu_{0}} \frac{\partial}{\partial t}\langle\mathbf{e} \times \mathbf{b}\rangle+\nabla \cdot\left[\left(\frac{\epsilon_{0}}{2}\left\langle\mathbf{e}^{2}\right\rangle+\frac{1}{2 \mu_{0}}\left\langle\mathbf{b}^{2}\right\rangle\right) \mathrm{I}-\left(\epsilon_{0}\langle\mathbf{e e}\rangle+\frac{1}{\mu_{0}}\langle\mathbf{b b}\rangle\right)\right]\right\}
$$

which is the ponderomotive force-density contribution; the first term results from the wave Poynting moment, the second is the gradient of a pure isotropic wave pressure, 
the third is related to wave pressure anisotropy. The inverse proportionality of this expression to the mass shows that the main contribution is due to electrons. Ions can be neglected as they are (in the non-relativistic case considered here) insensitive to ponderomotive effects. This term is responsible for anomalous collisions, anomalous resistivity and viscosity.

The above equations do not form a complete system of conservation laws unless the pressure is given. The pressure follows from the third-order moment equation, i.e. the energy conservation law. This is replaced by equations of state, $\mathrm{P}\left(N, \gamma, T_{\|}, T_{\perp}\right)$, which express the pressure tensor components through density, temperature, and adiabatic coefficient $\gamma$. This procedure truncates the infinite series of conservation laws. Equations of state in non-equilibrium are merely approximations which hold under isothermality - which does not apply to shocks as they are not in thermal equilibrium —or adiabaticity. The latter condition is quite reasonable in dealing with the fast processes in the shock environment when the flow passes across the shock front in a time so short that isothermality cannot be attained.

Hence, shock can at the best behave adiabatically. But numerical simulations show that even adiabaticity is violated in most cases.

\subsubsection{Jump conditions}

In the centre-of-mass frame, introducing the rescaled variables

$$
\begin{gathered}
m=\sum_{ \pm} m_{ \pm}=m_{i}\left(1+\frac{m_{e}}{m_{i}}\right), \quad N=\left(\sum_{ \pm} m_{ \pm} N^{ \pm}\right) / \sum_{ \pm} m_{ \pm} \\
\mathbf{V}=\left[\sum_{ \pm} m_{ \pm}(N \mathbf{V})^{ \pm}\right] / \sum_{ \pm} m_{ \pm} N^{ \pm}
\end{gathered}
$$

the fluid equations can be simplified, leading to the magnetogasdynamic (or MHD) equations for a single-fluid plasma. The equation of continuity remains unchanged from Eq. 14. It thus suffices to simply write down the momentum conservation equation

$$
\frac{\partial(m N \mathbf{V})}{\partial t}+\nabla \cdot(m N \mathbf{V V})=-\nabla \cdot \mathbf{P}+\rho \mathbf{E}+\mathbf{j} \times \mathbf{B}
$$

where $\mathrm{P}=\mathrm{P}_{e}+\mathrm{P}_{i}$ is the total pressure tensor, and $\rho$ is the electric charge density $\rho=e\left(N_{i}-N_{e}\right)$ which in quasi-neutral plasmas outside the shock is assumed to be zero such that the second term on the right containing the average electric field vanishes outside the shock ramp. The last term in this equation is the Lorentz force written in terms of the average current density from Ampère's law $\mu_{0} \mathbf{j}=\nabla \times \mathbf{B}$. These equations hold for very slow variations with frequency much less than the ion cyclotron frequency $\omega \ll \omega_{c i}=e B / m_{i}$, scales much larger than the ion gyro-radius $L \gg r_{c i}=V_{i \perp} / \omega_{c i}$, and wave speeds much less than the speed of light. Note that this equation is completely collisionless. If we would have retained the pseudo-collision term on the right in the electron equation this would simply have added an electron ponderomotive force term on the right. Completion of these equations with an equation 
of state and a relation between the current and the average electric field, i.e. with Ohm's law, is necessary. The latter is found by subtracting the electron and ion momentum conservation equations and turns out to be quite complicated (cf. e.g., Baumjohann and Treumann 1996). In slightly simplified form the collisionless Ohm's law reads

$$
e N(\mathbf{E}+\mathbf{V} \times \mathbf{B})=\mathbf{j} \times \mathbf{B}-\nabla \cdot \mathrm{P}_{e}+\left(m_{e} / e\right)(\partial \mathbf{j} / \partial t)
$$

The right hand side contains only electron terms. Also, an electron ponderomotive term-responsible for anomalous transport effects-would appear if we would retain the pseudo-collision term. However, even in this form the non-collisional Ohm's law is intractable for treating the conservation laws across the shock transition.

For flat shocks the terms on the right can be neglected, yielding the ideal Ohms law $\mathbf{E}=-\mathbf{V} \times \mathbf{B}$. The assumption of a flat shock implies that one resides far away from the shock transition at a location where the shock excited turbulence has decayed. Here the global conservation laws can be applied. The sole variation is then along the shock normal (Fig. 1), and the equations can be integrated along $\mathbf{n}$ across the shock (with regular boundary conditions at $x= \pm \infty$ ). Applying the prescription for the $\nabla$-operator in Eq. 5 these equations are transformed into a nonlinear algebraic set for the jumps $[\ldots]$ in the field quantities

$$
\begin{array}{r}
\mathbf{n} \cdot[N \mathbf{V}]=0, \quad[\mathbf{n} \times \mathbf{V} \times \mathbf{B}]=0, \quad \mathbf{n} \times[\mathbf{B}]=0 \\
\mathbf{n} \cdot[m N \mathbf{V V}]+\mathbf{n}\left[P+\frac{B^{2}}{2 \mu_{0}}\right]-\frac{1}{\mu_{0}} \mathbf{n} \cdot[\mathbf{B B}]=0 \\
{\left[m N \mathbf{n} \cdot \mathbf{V}\left\{\frac{V^{2}}{2}+w+\frac{1}{m N}\left(P+\frac{B^{2}}{\mu_{0}}\right)\right\}-\frac{1}{\mu_{0}}(\mathbf{V} \cdot \mathbf{B}) \mathbf{n} \cdot \mathbf{B}\right]=0}
\end{array}
$$

The last expression accounts for the conservation of energy. $w=c_{v} P / k_{B} N$ is the ideal gas enthalpy density, $c_{v}$ the specific heat, and $k_{B}$ Boltzmann's constant. This set of equations is the implicit form of the Rankine-Hugoniot conservation laws in ideal magnetogasdynamics (ideal MHD). It contains all MHD discontinuities of which shock waves are a subclass, the class of solutions with finite flow across the discontinuity, compressions (in density and magnetic field), and increases in temperature $T$, pressure $P$, and entropy $\mathscr{S}$ across the discontinuity when transiting from upstream to downstream.

Genuine shock jump solutions (see, e.g. Landau and Lifshitz 1959) of Eqs. 20 require finite mass flux $\mathscr{F}=N V_{n}$ in normal direction across the shock. The first Rankine-Hugoniot relation (20) tells that the jump $[\mathscr{F}]=0$. Hence $\mathscr{F}=$ const, and we must sort for solutions with $\mathscr{F} \neq 0$, or $N_{1} V_{n 1}=N_{2} V_{n 2}$, in order to deal with a shock. Introducing the specific volume $\mathscr{V}=(m N)^{-1}$, the whole system of jump conditions can be factorized (Baumjohann and Treumann 1996) and can be given the form

$$
\mathscr{F}\left(\mathscr{F}^{2}-\frac{B_{n}^{2}}{\mu_{0}\langle\mathscr{V}\rangle}\right)\left\{\mathscr{F}^{4}+\mathscr{F}^{2}\left(\frac{[P]}{[\mathscr{V}]}-\frac{\langle\mathbf{B}\rangle^{2}}{\mu_{0}\langle\mathscr{V}\rangle}\right)-\frac{B_{n}^{2}}{\mu_{0}\langle\mathscr{V}\rangle} \frac{[P]}{[\mathscr{V}]}\right\}=0
$$




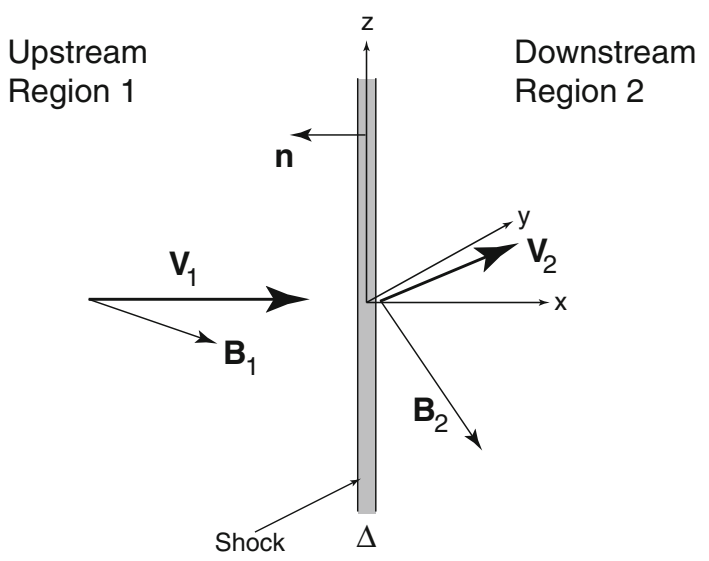

Fig. 1 A sketch of planar thin (width $\Delta$ ) shock geometry with $\mathbf{n}$ shock normal, upstream and downstream regions, bulk flow velocities and magnetic field vectors. Shocks are compressive. The downstream magnetic field is increased, while the normal velocity decreases. In addition, velocity and magnetic field are deflected at the shock. Deflection of the magnetic field is due to the conservation of the normal component $B_{n}$, while deflection of velocity is due to the presence of the obstacle and continuity of the tangential electric field (in an ideal upstream and downstream plasma) only

Magnetogasdynamic shock waves with $\mathscr{F} \neq 0$ are contained in the expression in curly braces which still depends on the prescribed jumps in pressure $[P]$ and specific volume $[\mathscr{V}]$ and thus on the equation of state. We will not discuss this equation further, as in the following more insight can be gained from explicit consideration of a few particular cases.

\subsection{Types and properties of collisionless shocks}

The physical property of the shock lies in its capability of slowing down the upstream flow from $\mathscr{M}>1$ to $\mathscr{M} \leq 1$, on the short distance $\Delta_{\text {sh }}$. A substantial amount of upstream flow energy must thereby be converted into compression and heating of the plasma, and increasing the pressure within $\Delta_{\text {sh }}$. The shock creates a downstream region of high thermal but low ram pressure and separates it from the low-thermalhigh-ram pressure upstream regime. Being a permeable boundary between two regions of different pressures, the shock is not in thermal equilibrium. Therefore, it cannot be stationary; in order to be maintained over long periods it must continuously reform at the expense of energy and momentum density inflow from the shock-upstream plasma.

\subsubsection{Electrostatic shocks}

Plasmas consist of electrically charged particles which under normal conditions have same number densities, $N_{e}=N_{i}=N$ in order to maintain overall charge neutrality. When the different charges, in the average, do not possess different bulk velocities, the plasma is free of electric currents $\mathbf{j}=N e\left(\mathbf{V}_{i}-\mathbf{V}_{e}\right)=0$ and, in the absence of an external magnetic field $\mathbf{B}_{0}$, the plasma is thus free of magnetic fields. In this case 
a shock wave which occurs in the flow is called an electrostatic shock. Electrostatic shocks are steep and small-scale local density perturbations. Their width is of the order of the Debye length $\lambda_{D}$, and their extension in tangential direction is of the order of the ion inertial length

$$
\lambda_{i}=c / \omega_{p i}=\sqrt{m_{i} / m_{e}}\left(c / v_{e}\right) \lambda_{D} \gg \lambda_{D}
$$

Even though electrostatic shocks are important for turbulent dissipation and may even contribute to radiation, they cannot be resolved remotely by any means. In astrophysics, electrostatic shocks can be attributed to the internal microscopic state of the matter.

\subsubsection{Magnetized shocks}

The vast majority of collisionless shocks are magnetized shocks simply, because most plasmas contain magnetic fields and allow for electric currents to flow across and along the magnetic field. Magnetized shocks behave quite differently from electrostatic shocks. One distinguishes between shocks in which the upstream magnetic field is tangential to the shock surface and shocks where the magnetic field is perpendicular to the shock surface. In the former case the upstream flow velocity, $\mathbf{V}_{1} \perp \mathbf{B}_{1}$, is perpendicular to the upstream magnetic field while, in the second case, it is parallel to the magnetic field, $\mathbf{V}_{1} \| \mathbf{B}_{1}$. In the perpendicular case the magnetic term $\mathbf{V}_{1} \times \mathbf{B}_{1} \neq 0$ in the Lorentz force is finite. The magnetic field lines are convected with the flow and pile up at the shock ramp. In the parallel case the magnetic term in the Lorentz force vanishes identically. From a naive point of view such shocks become unmagnetized and should behave like gasdynamic shocks. This is, however, not the case, as will become clear later. Defining the shock normal angle $\theta_{B n}$ through $^{8}$

$$
\tan \theta_{B n}=\mathbf{n} \cdot \mathbf{B}_{1} /\left|\mathbf{B}_{1}\right|
$$

perpendicular shocks have $\theta_{B n}=\frac{1}{2} \pi$, and parallel shocks have $\theta_{B n}=0$. These two extreme cases are realised at large-scale bent shocks only over small portions of the shock surface. One therefore distinguishes quasi-perpendicular and quasi-parallel shocks defined by

$$
\frac{1}{4} \pi<\theta_{B n} \leq \frac{1}{2} \pi \quad \text { and } \quad 0 \leq \theta_{B n}<\frac{1}{4} \pi
$$

respectively. In the overlap region $\frac{1}{6} \pi<\theta_{B n} \leq \frac{1}{3} \pi$ one speaks of oblique shocks, keeping in mind that, in contrast to the distinction between parallel and perpendicular shocks, the term oblique is conventional only and is not required by physics. Oblique shocks simply have mixed parallel/perpendicular shock properties.

\footnotetext{
${ }^{8}$ In the spirit of our earlier remarks, $\theta_{B n}$ is defined locally and depends on the existence of a well-defined shock normal $\mathbf{n}$, implying the assumption of a (sufficiently sharp) narrow and well-defined shock transition layer.
} 


\subsubsection{MHD shocks}

The simplest (and historically the first) model approach to collisionless shocks is in magnetohydrodynamic fluid theory. The equation of state between the plasma pressure P, density $N$ and temperature $T$ (in energy units) that completes the system is the ideal ${ }^{9}$ gas law $P=N T$ in its adiabatic version, assuming that shock formation proceeds on a short time scale such that the temperature cannot adjust. This is, in fact, not an unreasonable assumption as a shock cannot be in thermal equilibrium, as has been argued above on different reasons. In strong magnetic fields one takes into account the anisotropy of the pressure tensor $\mathrm{P}=P_{\|} \mathrm{I}+\left(P_{\perp}-P_{\|}\right) \mathbf{B B} / B^{2}$.

The three possible MHD-classes of magnetised shocks that follow from the solution of the shock part in the Rankine-Hugoniot conditions Eq. 21 are fast, slow and intermediate shocks. They are related to the three MHD wave modes. Their occurrence depends on which of the wave phase speeds is exceeded by the flow velocity, and for the slower shocks, of course, under the additional condition that the faster waves are inhibited in the medium. If the faster waves were to be excited in the interaction of flow and obstacle, they would propagate faster upstream than the (slow or intermediate) shock itself and inform the flow about the presence of the obstacle. No shock would be formed in this case from the slower Alfvénic or slow modes. Since this is the more realistic case, the most frequently observed shocks are fast shocks. (Occasionally, also intermediate and slow shocks have been claimed to have been detected in interplanetary space, however.) The four possible shock transitions in terms of the relations between flow and MHD wave mode speeds (De Hoffman and Teller 1950; Balogh and Riley 2005) are:

$$
\begin{array}{ll}
\operatorname{trans} 1: V>c_{m s}^{+}, & \operatorname{trans} 2: c_{m s}^{+}>V>c_{i n t} \\
\operatorname{trans} 3: c_{i n t}>V>c_{m s}^{-}, & \operatorname{trans} 4: c_{m s}^{-}>V
\end{array}
$$

where the velocities $c_{m s}^{ \pm}$have been defined in Eq. 3, and $c_{i n t}=V_{A} \cos \theta$ is the angle-dependent Alfvén velocity of the intermediate wave. Not all these transitions can, however, be realized. Entropy considerations allow only for the transitions $1 \rightarrow$ $2,1 \rightarrow 3,1 \rightarrow 4,2 \rightarrow 3,2 \rightarrow 4$, and $3 \rightarrow 4$ (Wu and Kennel 1992), as only in these directions are compression and heating possible, which would be the requirement for the increase in entropy. Of these, the first is a fast mode shock, while the transition $3 \rightarrow 4$ is a slow mode shock, and the remainder are intermediate shocks which, together with the slow mode shock, might sometimes exist under the above mentioned restrictions. Usually an obstacle will excite all three waves together, and then only the fast mode will cause a shock. The observation of slow or intermediate shocks thus requires very special conditions to be realised in the plasma. In addition,

\footnotetext{
9 In all fluid plasma theory the assumption is made that one is dealing with an ideal gas. This assumption has turned out to be useful, but has not been justified. In particular, in (single fluid) MHD the smallest perpendicular scale on which the equations hold is the (thermal) ion gyroradius $r_{c i}=v_{i} / \omega_{c i}$. This, formally, should imply that transverse pressures in MHD encounter a rigid magnetic flux tube at this scale. One may expect that one is forced to replace the perpendicular ideal gas equation by a Van der Waals equation. This is of course not the case in this rigour as the MHD approximation breaks down here and one has to include finite gyro- radius effects or use kinetic theory.
} 
any MHD shocks will have to respect the evolutionary conditions which we discuss briefly below.

In the MHD frame one can define simple relations between the parameters of the streaming gas upstream and downstream of the shock, the so-called Rankine-Hugoniot relations. The restriction on them is that the processes which determine the generation of entropy that cause the irreversibility of the shock must all be strictly confined to the shock transition. This is not as easy to achieve as it might seem at first glance. The shock transition might be much broader and more extended than the proper shock ramp suggests. Hence the parameter values entering the Rankine-Hugoniot relations are correctly taken only from far away to both sides of the shock ramp in order to assure that dissipative processes are not mixed-in. The problem is then that the shock surface itself must be extended enough compared with the distance from the shock where the parameter values are taken. In addition, its curvature should still be negligible in order to not destroy the assumption of shock planarity.

Under the special condition that the flow in the upstream Region 1 is along $x$ (antiparallel to $\mathbf{n})$ and the upstream magnetic field $\mathbf{B}_{1}=\left(B_{1 x}, 0, B_{1 z}\right)$ is in the $x z$-plane, the shock jump condition Eqs. 21 simplifies substantially. In normalised downstream variables $N_{2} / N_{1} \rightarrow N_{2}, \mathbf{V}_{2} / V_{1} \rightarrow \mathbf{V}_{2}, T_{1,2} /\left(m V_{1}^{2} / 2\right) \rightarrow T_{1,2}, \mathbf{B}_{1,2} / \sqrt{\mu_{0} m N_{1} V_{1}^{2}} \rightarrow$ $\mathbf{B}_{1,2}$ it becomes a cubic equation

$$
a_{3} V_{n 2}^{3}+a_{2} V_{n 2}^{2}+a_{1} V_{n 2}+a_{0}=0
$$

where $a_{0}=-B_{n}^{2}\left[B_{z 1}^{2}+B_{n}^{2}\left(1+5 v_{1}^{2}\right)\right], a_{1}=2 B_{n}^{2}\left(1+2 \mathbf{B}_{1}^{2}+5 v_{1}^{2}\right)-\frac{1}{2} B_{z 1}^{2},-a_{2}=$ $1+5 v_{1}^{2}+8 B_{n}^{2}+\frac{5}{2} B_{z 1}^{2}, a_{3}=4$. The simplest case is a strictly perpendicular shock with $\theta_{B n}=\pi / 2$ and $B_{n}=0, B_{z 1}=B_{1}, a_{0}=0, a_{1}=-\frac{1}{2} B_{1}^{2},-a_{2}=1+5 v_{1}^{2}+\frac{5}{2} B_{z 1}^{2}, a_{3}=$ 4 , and the jump condition (25) simplifies further, becoming a quadratic equation. It has the solution

$$
8 V_{n 2}=1+\left(1+2.5 \beta_{1}\right) B_{1}^{2}+\left\{\left[1+\left(1+2.5 \beta_{1}\right) B_{1}^{2}\right]^{2}+2 B_{1}^{2}\right\}^{\frac{1}{2}}=8 N_{2}^{-1}=8 B_{2}^{-1}
$$

The condition for a shock to exist is that the normal velocity $V_{n 2}<1$ in Region 2 , and we immediately conclude that in a perpendicular shock the density and tangential magnetic field components in Region 2 increase by the same fraction as the normal velocity drops. This fraction is determined by the plasma- $\beta$ ratio $\beta_{1}=2 \mu_{0} N_{1} T_{1} / B_{1}^{2}$ in Region 1, where $T_{1}=T_{e 1}+T_{i 1}$ is the total temperature. The condition on $V_{n 2}$ implies that the Mach number and temperature take the form (now in physical units)

$$
1<\mathscr{M}=\frac{1}{1+5 \beta_{1} / 6} \mathscr{M}_{A}, \quad \frac{T_{2}}{T_{1}}=1+\frac{2}{5 T_{1}}\left[1-N_{2}^{-2}+2 \mathscr{M}_{A}^{-2}\left(1-N_{2}\right)\right]>1
$$

Here $\mathscr{M}_{A}=V_{1} / V_{A 1}$ is the Alfvén-Mach number which is the flow to Alfvén velocity ratio. In cold plasmas (or plasmas containing strong magnetic fields) one has $\beta \ll 1$, and the Mach number is simply the Alfvén-Mach number. Conversely, in hot plasmas 
the Mach number becomes about the ordinary gasdynamic Mach number. The temperature ratio is always larger than one. Perpendicular shocks cause plasma heating in the shock transition time. The entropy increases by $\Delta \mathscr{S} \propto \ln \left[\left(T_{2} / T_{1}\right)^{1 /(\gamma-1)} N_{2}^{-1}\right]$. This holds under the ideal gas assumption.

\subsubsection{Evolutionarity}

Not all of the six possible MHD-shock solutions can be realized. The actual reason for this lies in the evolutionarity conditions of shock waves, which are based on the hyperbolic nature of the conservation laws. These allow wave propagation only if it is in accord with causality (Lax 1957).

For MHD waves with dissipation these conditions have been discussed by Jeffrey and Taniuti (1964), Kantrowitz and Petschek (1966), Liberman and Velikhovich (1986) and others. They hold also in the collisionless regime. Causality in this case means that the drop in speed across a shock (in the wave mode that causes the shock) must be large enough for the normal component of the downstream flow to drop below the corresponding downstream mode velocity. For a fast shock this implies the following ordering of the normal flow and magnetosonic velocities to both sides of the shock:

$$
V_{1 n}>c_{1 m s}^{+}, \quad \text { while } \quad V_{2 n}<c_{2 m s}^{+}
$$

As before, the numbers 1, 2 refer to upstream and downstream of the fast shock wave. The first condition is necessary for the shock to exist; it is the second condition which accounts for evolutionarity. ${ }^{10}$ Otherwise the small fast mode disturbances excited downstream and moving upwards towards the shock would move faster than the flow, they would overcome the shock and steepen it without limit. Since this should not happen, the downstream normal speed must be less than the downstream fast magnetosonic speed. Similar conditions hold for any shock as, also, for large amplitude shocks. Furthermore, for fast shocks the flow velocity must be greater than the intermediate speed on both sides of the shock, while for slow shocks it must be less than the intermediate speed on both sides, otherwise the corresponding waves would catch up with the shock front, modify and destroy it and no shock could form.

\subsubsection{Coplanarity}

Another property of MHD shocks relates to the directions of the magnetic field and flows to both sides of the shock front. In MHD these directions are not arbitrary. It can be shown from the Rankine-Hugoniot relations that the flow and magnetic field directions in front and behind the shock front in MHD lie in the same plane, i.e. they are coplanar. This property had been realized already by Marshall (1955) and was discussed in depth by Kantrowitz and Petschek (1966) and others (e.g. Burgess 1995). For a stationary ideal MHD shock wave the electric field in the shock rest frame is strictly perpendicular to the magnetic field. Faraday's law $\nabla \times \mathbf{E}=0$ and the shock normal $\mathbf{n}$ yield that the scalar product between $\mathbf{n}$ and the difference in the tangential

$\overline{10}$ We have, in fact, already made use of this condition in Eq. 27 for a strictly perpendicular MHD shock. 
components of the magnetic field to both sides $\left(V_{n 2}-V_{n 1}\right)\left\{\mathbf{B}_{\mathrm{t} 2} \times \mathbf{B}_{\mathrm{t} 1}\right\}=0$ vanishes. The difference in the normal components across the shock is finite, such that $V_{n 2} \neq V_{n 1}$. Hence

$$
\mathbf{n} \cdot\left\{\mathbf{B}_{2}-\mathbf{B}_{1}\right\}=0
$$

which implies not only that $\mathbf{n}$ is normal to the tangential components of the magnetic field on both sides of the infinitesimally thin discontinuity, which would be a trivial conclusion, but also that the two tangential components to both sides are strictly parallel. They may — and should—have different lengths but will have the same direction across the shock.

Coplanarity does not hold strictly, however. When the shock is nonstationary (e.g. when its width changes with time, which is excluded by MHD as all such processes are contained in the internal structure of the dissipation region of width $\Delta$, and this is determined by processes not covered in MHD), the right-hand side in Faraday's law does not vanish, and coplanarity may become violated. Also, any upstream low frequency electromagnetic plasma wave that propagates along the upstream magnetic field possesses a magnetic wave field that is perpendicular to the upstream field. When it encounters the shock, its component that is tangential to the shock will be transformed and amplified across the shock. This naturally introduces an out-of-plane magnetic field component, thereby violating the co-planarity condition.

\subsubsection{Criticality}

The most important property of collisionless shocks is their criticality. ${ }^{11}$ It introduces a further-physically motivated - classification into subcritical and supercritical shocks according to their Mach-numbers $\mathscr{M}<\mathscr{M}_{c}$ being smaller, or $\mathscr{M}>\mathscr{M}_{c}$ being larger than some critical Mach-number $\mathscr{M}_{c}$. For a resistive shock the numerically determined (Marshall 1955) largest critical Mach number is

$$
\mathscr{M}_{c} \approx 2.76
$$

The dependence of the critical Mach number on plasma $\beta$ and $\theta_{B n}$ is shown in Fig. 2. Critical Mach numbers are small and decrease with increasing shock normal angle $\theta_{B n}$. Since the accessible nonrelativistic (or weakly relativistic) astrophysical shocks have Mach numbers of the order of $\sim 10^{3}$ or larger and, in addition, occur in high- $\beta$

\footnotetext{
11 Criticality refers to the capability of a shock wave to maintain its shock character solely by resistive (Joule) dissipation. Since collisionless shocks are free of binary interaction between the particles, any resistance must be anomalous. Consequently, criticality is defined with respect to anomalous Joule dissipation. It depends on the mechanism of generation of anomalous effects. In this sense it is defined implicitly only, because the generation of anomalous dissipation is a property of the shock itself. Strictly speaking, a collisionless shock that does not generate any anomalous dissipation is supercritical for all Mach numbers $\mathscr{M}>1$. Usually, a collisionless shock generates some weak dissipation, however. Therefore, it makes sense to define a largest critical Mach number $\mathscr{M}_{c}$ by assuming a resistivity. It turns out that the maximum critical Mach number $\mathscr{M}_{c}$ is independent of the value of the resistivity and is determined by the incapability of the shock for $\mathscr{M}>\mathscr{M}_{c}$ to generate the entropy increase that is required to maintain the shock.
} 

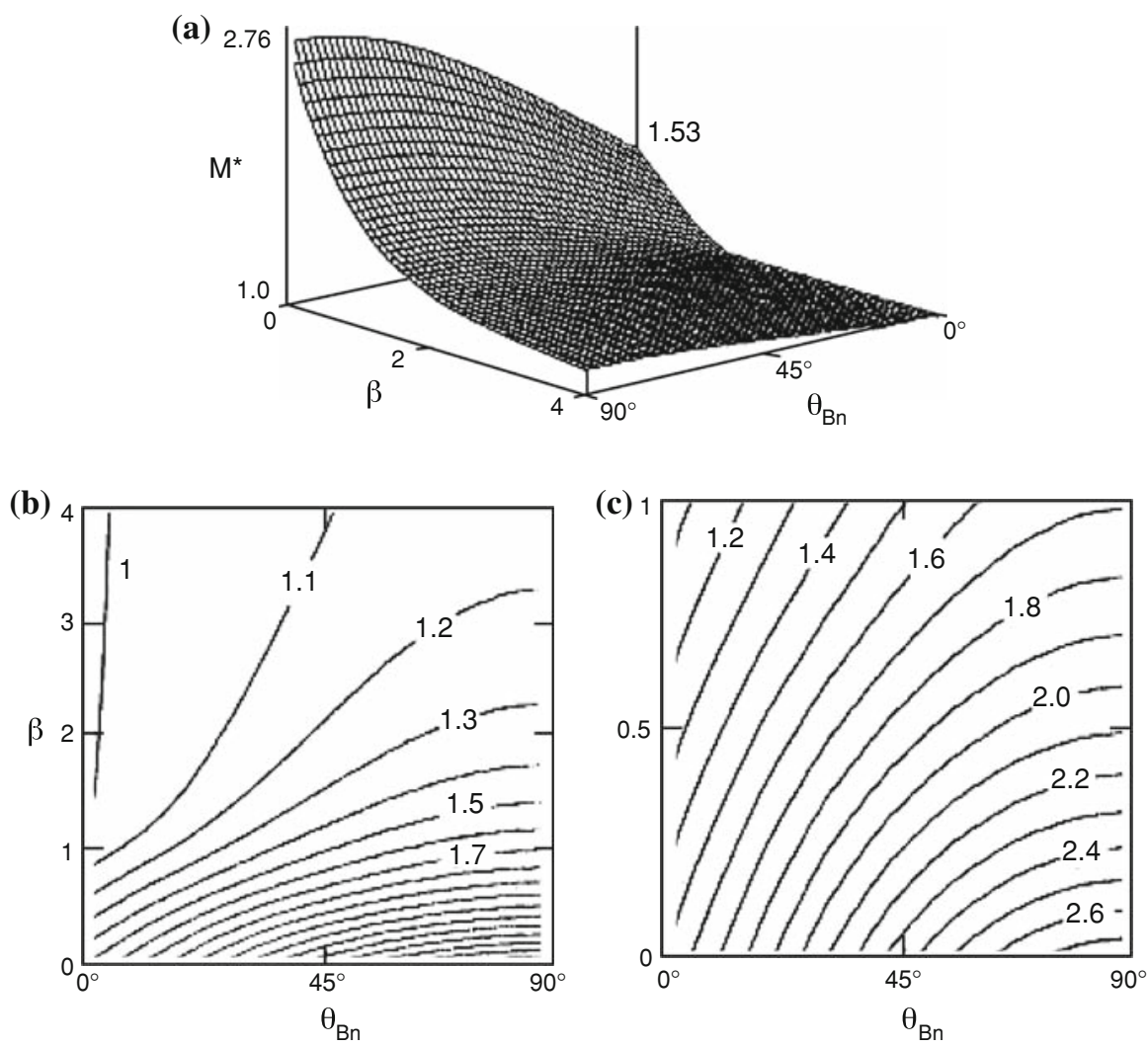

Fig. 2 Parametric dependence of the critical Mach number $\mathscr{M}_{c}$ for a fast shock on the upstream plasma- $\beta_{1}$ (top) and shock angle $\theta_{B n}$ for the special case of adiabatic $\gamma=\frac{5}{3}$. The lower panels show two $\beta_{1}$ ranges of critical Mach number contours (from Edmiston and Kennel 1984). For large $\beta_{1}>1$ the critical Mach number is close to $\mathscr{M}_{c} \sim 1$, while for smaller $\beta_{1}$ it is a strong function of $\theta_{B n}$ having its lowest value $\mathscr{M}_{c}=1.53$ for parallel and $\mathscr{M}_{c}=2.76$ for perpendicular shocks. The latter value is the same as that originally inferred by Marshall (1955)

environments, the overwhelming majority of astrophysical shocks is highly supercritical.

Subcritical shocks are capable of generating sufficient dissipation to account for the required retardation, thermalization and entropy increase. This dissipation is confined to the shock transition and is generated during the crossing time of a fluid element from upstream to downstream. The relevant processes are based on wave-particle interaction between the shocked plasma population and the shock-excited turbulent wave fields. Supercritical shocks must evoke mechanisms different from simple waveparticle interaction for getting rid of the excess energy in the bulk flow that cannot be dissipated by any anomalous dissipation. Above the critical Mach number $\mathscr{M}_{c}$ the simplest efficient way of energy dissipation of the excess energy is on reflecting a substantial part of the inflowing plasma back upstream.

The critical Mach number can be inferred from consideration of the maximum possible dissipation. For dissipative shocks, the shock width $\Delta_{\text {sh }}$ must exceed the mag- 


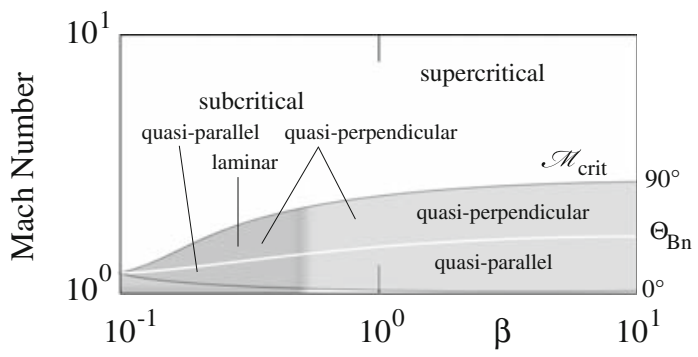

Fig. 3 The range of supercritical and subcritical shocks in the $\left(\mathscr{M}_{m s}, \beta\right)$-plane. The critical Mach number range is shown in shading varying between perpendicular and parallel shocks. The white line is the demarcation line between quasi-perpendicular and quasi-parallel shocks. The lower dark line belongs to strictly parallel shocks. At low $\beta$ the parameter range of laminar shocks is shown in fuzzy shading. The transition to higher beta is blurred as it is not sharply defined. For a given shock normal angle $\theta_{B n}$ the region below the curve is subcritical. Quasi-parallel shocks can be subcritical only below the white line, depending on their shock normal angle

netic Reynolds length. The observed finite magnetic field compression-ratio therefore imposes an upper limit to the possible rate of resistive dissipation in an MHD shock (Kantrowitz and Petschek 1966; Coroniti 1970). When the wavelength of the fast wave is equal to the resistive length, the magnetic field decouples by resistive dissipation, and the wave speed becomes the sound speed $c_{2 s}$ downstream of the shock ramp. The condition for the critical Mach number is then given by $V_{2 n}=c_{2 s}$. Since these quantities depend on wave angle they have to be solved numerically (Edmiston and Kennel 1984). The 'first' critical Mach number varies between 1 and 2.76, depending on the upstream plasma parameters and flow angle to the magnetic field. There is theoretical evidence in simulations for a 'second' critical Mach number which comes into play when whistlers accumulate at the shock front and periodically cause its reformation. Then the dominant dispersion is the dispersion of whistler waves. An approximate expression for this second or whistler critical Mach number is

$$
\mathscr{M}_{2 c} \propto \sqrt{m_{i} / m_{e}} \cos \theta_{B n}
$$

The constant of proportionality depends on whether one defines the Mach number with respect to the whistler phase or group velocities. For the former it is $\frac{1}{2}$, and for the latter slightly less, $\sqrt{27 / 64}$ (e.g. Oka et al. 2006).

The smallest critical Mach number determines the behaviour of the shock. In simple words: $\mathscr{M}>1$ is responsible for the existence of the shock under the condition that an obstacle stands in the flow. When, in addition, the flow exceeds the next lowest critical Mach number for a given $\theta_{B n}$, the shock becomes supercritical at this angle and starts reflecting particles back upstream. Exceeding the second critical Mach number should cause quasi-periodic whistler-driven shock reformation. 


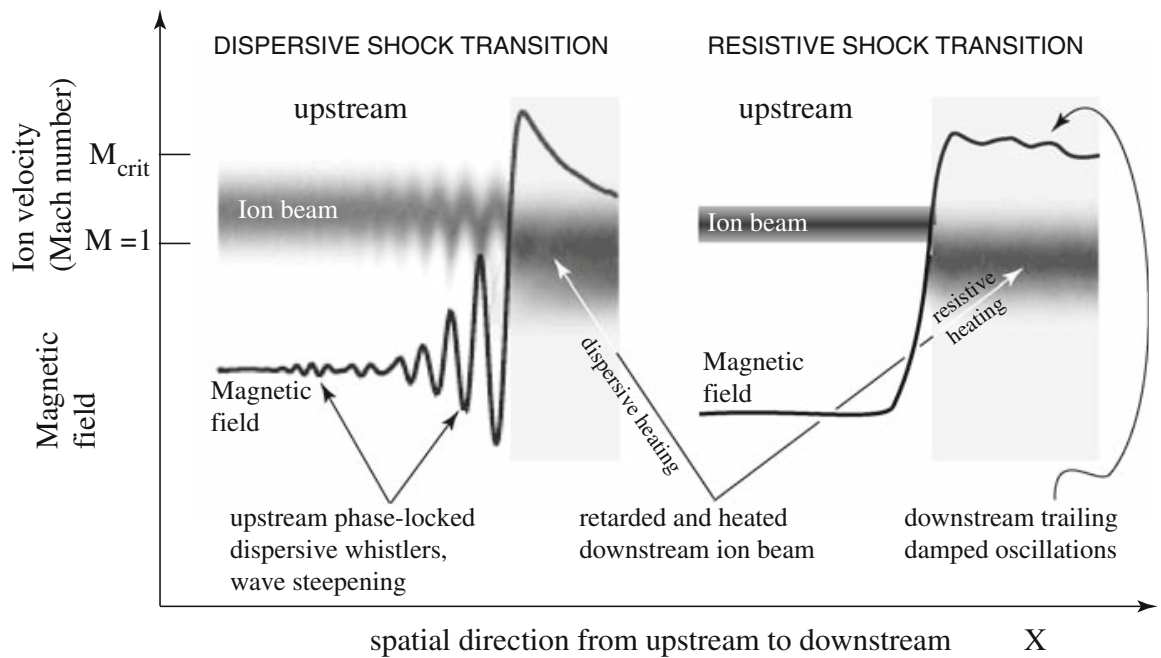

Fig. 4 Schematic of the two types of subcritical shocks in ion phase space and magnetic field profile. In the dispersive shock transition the upstream whistler steepens nonlinearly while being phase-locked to the shock and causing coherent oscillations in the upstream ion beam. The upstream ion beam is subcritical. Heating and retarding in the shock is caused by dispersive effects. In resistive shock transition the shock forms a steep ramp lacking upstream oscillations. Heating and retarding is due to resistive Joule heating. Downstream the magnetic field may evolve trailing oscillations. Other shock transitions based on the Korteweg-deVries and related equations are also possible

\section{Collisionless subcritical shocks}

Below the critical Mach number $\mathscr{M}<\mathscr{M}_{c}$ shocks are subcritical. Since $\mathscr{M}_{c}$ is a function of $\beta$ and $\theta_{B n}$, depending on their Mach numbers, shocks with curved shock surfaces are subcritical only over a narrow section of their surface. Figure 3 shows how narrow the range is of collisionless subcritical shocks in the Mach number-plasma- $\beta$ space. For parallel shocks a noticeable subcritical range exists only at low upstream beta $\beta \lesssim 0.5$. Between perpendicular and parallel shocks there is a broader transition region where quasi-perpendicular shocks are still subcritical while quasi-parallel shocks are already supercritical. Laminar quasi-perpendicular shocks can presumably be found only at small $\beta$. At larger $\beta$ they become more and more fluctuating. The restriction to these low Mach numbers substantially limits the application of subcritical shocks to astrophysical conditions. Some remarks on this will be found in the concluding Sect. 2.4.

In contrast to supercritical shocks, ${ }^{12}$ subcritical collisionless shocks require the presence of some kind of anomalous dissipation inside the shock transition. Thus, the main problem in subcritical shocks consists in the identification of the relevant anomalous dissipation process. In subcritical shocks the cooperation of dissipation inside the shock front and dispersion of waves is sufficient for providing the necessary

\footnotetext{
12 See Sects. 3 and 4, where the main dissipative process is particle reflection, such that, in principle, supercritical shocks could exist in the complete absence of any conventional dissipation.
} 
dissipation/dispersion of sustaining the shock transition from upstream to the downstream flow. For illustration, Fig. 4 shows two schematic subcritical shock profiles, one of them a dispersion dominated shock transition resulting from nonlinear steeping of waves (in this case whistlers), the other the extreme case of a purely resistive shock transition. Dispersion alone cannot create a shock transition. It produces some kind of localised waves like the various kinds of solitary waves and BGK modes. These are all structures that are connected with electric potential wells. Depending on the polarity of these potentials they reflect one sort of particle out of the upstream low energy component while they trap and accelerate particles from the other component. Hence, in the complete absence of any kind of dissipation, a subcritical 'shock' can exist only when it reflects and traps some particles. Then, however, it is a marginal case of shock, a non-dissipative structure which by definition is reversible. Such structures belong to the family of solitary waves. If this is not the case, the subcritical shock must be capable of generating anomalous collisionless dissipation even when it is dominated by dispersion, like in the first case shown. Processes that are capable of doing this have been proposed for long time (Karpman and Sagdeev 1964; Karpman 1964; Sagdeev 1966).

\subsection{Theory}

Early summaries of the theory of subcritical shocks can be found in the classical text of Zeldovich and Raizer (1966), the monograph by Tidman and Krall (1971), and the reviews by Sagdeev (1966) and Biskamp (1973). According to this theory the evolution of subcritical shocks is due to the competition between the nonlinear steeping of a large amplitude low frequency plasma wave and the dispersive properties of the plasma. Dispersion can lead to the formation of localised waves of soliton-type. In a medium that contains a small amount of dissipation the waves become weakly damped, and these localised structures evolve into a ramp which mimics a shock. It is important to note that the dissipation is in many cases quite unimportant as long as the amplitude and the steepness of the wave packet remain small. However when both increase, the gradient scale enters the scale of local dissipative interactions, and dissipation starts becoming important. Before constructing a model of the subcritical shock we therefore discuss the shock potential in a subcritical shock and the relevant scale of dissipation.

\subsubsection{Subcritical shock potential}

Subcritical shocks do not reflect ions. This means that all upstream ions pass the shock. However, the shock ramp, being of the order of the ion inertial length $\Delta_{\mathrm{sh}} \sim \lambda_{i}$, allows for the ions to be non-magnetised while the electrons are tied to the magnetic field. As a consequence, the shock ramp contains a finite electric potential $U$ which retards the ions, however is not large enough to stop their motion and to reflect them. Neglecting any upstream thermal spread of the ions, which move at upstream bulk velocity $V_{1}$, the ion speed $v_{x}$ across the shock is therefore given by 


$$
v_{x}(x)=\left[V_{1}^{2}-2 e U(x) / m_{i}\right]^{\frac{1}{2}}
$$

independent of how complicated the real motion of the ions would be in crossing the ramp. For a cold upstream flow with $\beta \ll 1$ this is also the bulk flow velocity inside the ramp. Flux conservation then yields that the local density becomes

$$
N(x)=N_{1}\left[V_{1} / v_{x}(x)\right]=N_{1}\left[1-2 e U(x) / m_{i} V_{1}^{2}\right]^{-\frac{1}{2}}
$$

The transmitted ion density is trivially related to the shock potential which, thus, can be determined from a single measurement of the transmitted ion density. On the other hand, scalar pressure balance requires that $m_{i} N v_{x}^{2}+P+B^{2} / 2 \mu_{0}=$ constant, $\left(P \equiv P_{i}+P_{e}\right)$. The (scalar) pressure increases when the particles in the ramp are heated. Substituting for $v_{x}$, the above relation can be used to express the shock ramp density and ion pressure (Gedalin 1997)

$$
\frac{N(x)}{N_{1}}=\frac{1}{[1-\bar{U}(x)]^{\frac{1}{2}}}\left\{1+\frac{3}{4} \frac{\beta_{i}}{\mathscr{M}_{A}^{2}} \frac{\bar{U}(x)}{[1-\bar{U}(x)]^{2}}\right\}, \quad \frac{P_{i}(x)}{P_{1 \mathrm{ram}}}=\frac{\beta_{i} / \mathscr{M}_{A}^{2}}{[1-\bar{U}(x)]^{\frac{3}{2}}}
$$

Here, the normalised potential $\bar{U} \equiv 2 e U / m_{i} V_{1}^{2}$ and upstream flow ram pressure $P_{1 \text { ram }}=\frac{1}{2} m_{i} N_{1} V_{1}^{2}$ have been introduced. This is an implicit expression for the shock potential $\bar{U}(x)$ at position $x$ in the shock ramp as a function of the local plasma density $N(x)$ and Mach number $\mathscr{M}_{A}$. At the top of the ramp the ions have velocity $V_{\mathrm{R}}\left(x_{\mathrm{R}}\right) / V_{1}=\left(1-\bar{U}_{\text {tot }}\right)^{\frac{1}{2}} \cdot \bar{U}_{\text {tot }}$ is the total normalised ramp potential drop. The corresponding velocity is less than the upstream speed but does not coincide with the downstream velocity $V_{2}$ determined from the Rankine-Hugoniot relations. The difference $V_{\mathrm{R}}-V_{2}$ gives the downstream gyration speed and the downstream ion temperature $T_{2} \sim m_{i}\left(V_{\mathrm{R}}-V_{2}\right)^{2} / 2$. The potential drop obeys the condition $\bar{U}<1-V_{2}^{2} / V_{1}^{2}$. Ion deceleration in the ramp is solely due to the shock potential, but the flow deceleration is related to the heating of the ions across the shock.

The shock ramp sees the ions unmagnetised. The electrons, on the other hand, are magnetised. They gyrate and experience the shock potential as a transverse electric field. Thus, inside the ramp the electrons undergo an $\mathbf{E} \times \mathbf{B}$-drift along the shock which generates an electron drift current $\mathbf{j}_{e d}(x)=e N(x) \nabla_{x} U(x) \times \mathbf{B}(x) / B^{2}(x)$ in the direction perpendicular to the shock ramp electric and magnetic fields. The magnitude of the tangential velocity $v_{e d} \equiv v_{e y}$ of this electron drift current (with $\xi=x / \lambda_{i}$ the normalised coordinate in shock normal direction) expressed in terms of the local shock potential $\bar{U}(\xi)$ is

$$
\frac{v_{e y}}{V_{A 1}}=\frac{\mathscr{M}_{A}^{2}}{2} \frac{B_{1}}{B(\xi)}\left|\frac{\partial \bar{U}(\xi)}{\partial \xi}\right|
$$

The direction of electron flow is along the shock. The magnetic field of this current causes a magnetic overshoot in the subcritical shock. In addition, if the electron current 
becomes strong enough it serves as source of instability and generates the required dissipation.

\subsubsection{Dissipation length}

For a subcritical shock the shock transition must be capable of generating sufficient dissipation to come up for the entropy increase in the flow when it crosses the shock. From the induction equation

$$
\partial \mathbf{B} / \partial t=\nabla \times \mathbf{V} \times \mathbf{B}-\left(\eta_{\mathrm{an}} / \mu_{0}\right) \nabla^{2} \mathbf{B}
$$

follows that dissipation dominates the flow if the resistive term is larger than the convection term. The shock has thickness $\Delta_{\text {sh }}$, and the flow has velocity $V_{1}$. Dissipation is active only during the convection time $\tau_{\mathrm{sh}}=\Delta_{\mathrm{sh}} / V_{1}$ needed for crossing the shock layer, i.e. the dissipation time $\tau_{d}=L_{d}^{2} \mu_{0} \eta_{\text {an }}^{-1}<\tau_{\text {sh }}$ must be shorter than the crossing time. Alternatively, the 'dissipation scale' $L_{d}<\Delta_{\text {sh }}$ is shorter than the shock width. ${ }^{13}$ Dimensionally, we obtain $L_{d}^{2} \lesssim\left(\eta_{\mathrm{an}} / \mu_{0}\right)\left(\Delta_{\mathrm{sh}} / V_{1}\right)$ for the dissipation scale. Expressing the anomalous resistivity in terms of the anomalous collision frequency $\nu_{\text {an }}$ and the electron inertial length $\lambda_{e}=c / \omega_{p e}$ yields, for the resistive length scale, $L_{d} \lesssim\left[v_{\mathrm{an}} /\left(V_{1} / \Delta_{\mathrm{sh}}\right)\right]^{\frac{1}{2}} \lambda_{e}$.

We now require that the dissipation scale be at most of the order of the shock width, $L_{d}=\sqrt{\alpha} \Delta_{\mathrm{sh}}$. Then it becomes a condition on the anomalous collision frequency

$$
\nu_{\mathrm{an}} \gtrsim \alpha\left(\frac{V_{1}}{\lambda_{e}}\right)\left(\frac{\Delta_{\mathrm{sh}}}{\lambda_{e}}\right) \quad \text { or equivalently } \quad \nu_{\mathrm{an}} \tau_{\mathrm{sh}} \gtrsim \alpha\left(\frac{\Delta_{\mathrm{sh}}}{\lambda_{e}}\right)^{2}
$$

where $\alpha \lesssim 1$ is a numerical factor of proportionality. For anomalous dissipation to be sufficiently large to sustain the shock, the second version of this condition suggests that the ratio of transition-time to collision-time must thus be larger than a fraction $\alpha$ of the square of the shock width measured in electron inertial lengths.

As an example, let us assume that the shock width is $\Delta_{\mathrm{sh}}=1,000 \mathrm{~km}$. Then, for a plasma density of $N \sim 5 \times 10^{6} \mathrm{~m}^{-3}$ and a subcritical flow velocity of $V_{1}=100 \mathrm{~km} / \mathrm{s}$, the anomalous collision frequency should be larger than $v_{\mathrm{an}}>10^{4} \alpha \mathrm{Hz}$, which is of an order similar to the electron plasma frequency $f_{p e} \sim 20 \mathrm{kHz}$. Since such high anomalous collision frequencies are unrealistic, one must require $\alpha \sim 0.1$, corresponding to a substantially narrower dissipation scale $L_{d} \sim 0.3 \Delta_{\text {sh }}$ or, correspondingly, narrower current sheets inside the shock transition.

If the subcritical shock evolves out of a magnetosonic solitary wave, the requirement on the anomalous collision frequency can also be expressed in terms of the upstream shock Mach number. For magnetosonic shocks there is a distinct relation between the

\footnotetext{
13 The dissipation scale is not the same as the mean free path of the particle which is $\lambda_{\operatorname{mfp}}=1 /\left(\sigma_{\text {coll }} N\right)$. In collisionless plasma $\lambda_{\mathrm{mfp}} \rightarrow \infty$. In contrast, the dissipation scale is the scale of the microscopic dissipation processes that are 'internal' to the shock transition and are responsible for the generation of the anomalous resistance $\eta_{\mathrm{an}}$. If dissipation is based on Joule heating, these processes are believed to be current-driven instabilities driven by the electric currents flowing in the shock transition layer.
} 
shock width and the Mach number (see, e.g., Sagdeev 1966) given approximately by $\Delta_{\text {sh }} \simeq \lambda_{e} / \sqrt{\mathscr{M}_{A}-1}$. Inserting into the first of the above conditions and noting that $\lambda_{e}^{2} / V_{A}^{2}=\left(m_{e} / m_{i}\right) \omega_{c i}^{-2}$, one obtains

$$
\frac{\nu_{\mathrm{an}}}{\omega_{c i}} \gtrsim \alpha\left(\frac{m_{i}}{m_{e}}\right)^{\frac{1}{2}} \frac{\mathscr{M}_{A}^{2}}{\sqrt{\mathscr{M}_{A}-1}}
$$

Similar relations also hold for other types of solitons. Assuming a subcritical Mach number of $\mathscr{M}_{A}=1.5$ this expression yields $\nu_{\text {an }}>140 \alpha \omega_{c i} \sim 3 \alpha \omega_{l h}$, a value still quite high for this kind of soliton, because anomalous collision frequencies will barely exceed the lower-hybrid frequency $\omega_{l h}=\sqrt{m_{e} / m_{i}} \omega_{c e}$. Subcritical current sheets in the shock transition layer will be narrower than the shock transition, mostly of the order of the electron skin depth $\lambda_{e}=c / \omega_{p e}$ implying that the currents are due to magnetised electrons flowing inside the shock transition. In view of our introductory remarks it is the steepness of the localised wave packets which is responsible for the generation of the dissipation.

\subsection{Origin of dissipation}

\subsubsection{Dispersion and shock wave steeping}

There is no problem with the understanding of dispersion in plasma. Shock waves grow out of waves that are generated in the impact of the super-magnetosonic flow onto the obstacle. The obstacle reflects ions back upstream, thus creating an ion-ion beam situation which is unstable with respect to low-frequency magnetosonic waves propagating upstream with velocity $V$ away from the obstacle. Under collisionless conditions their velocity evolves with time according to

$$
\mathrm{d} V / \mathrm{d} t=(\partial V / \partial t)+V \partial V / \partial x=0
$$

During propagation, the main effect on the shape of a sinusoidal disturbance $V \sim$ $\sin k\left(x-c_{m s} t\right)$ comes from the action of the nonlinear term. This term can be written as $V k \cos k\left(x-c_{m s} t\right)$. Inserting for $V$ this becomes $\sim \frac{1}{2} \sin 2 k\left(x-c_{m c} t\right)$. Hence, harmonic sidebands of half the wavelength and half the amplitude are generated which, by the same mechanism, also generate sidebands on their own, now at quarter original wavelength and amplitude, and so on, with increasingly shorter wavelengths. The total amplitude is the superposition of all these sideband harmonics which propagate at the same magnetosonic velocity $c_{m s}$. They superimpose locally and add to the wave amplitude, causing the wave to steepen until the gradient lengths become so short that dissipation takes over. If this does not happen, the wave will turn over and break, as illustrated in Fig. 5 in the co-moving frame of a sinusoidal wave. 

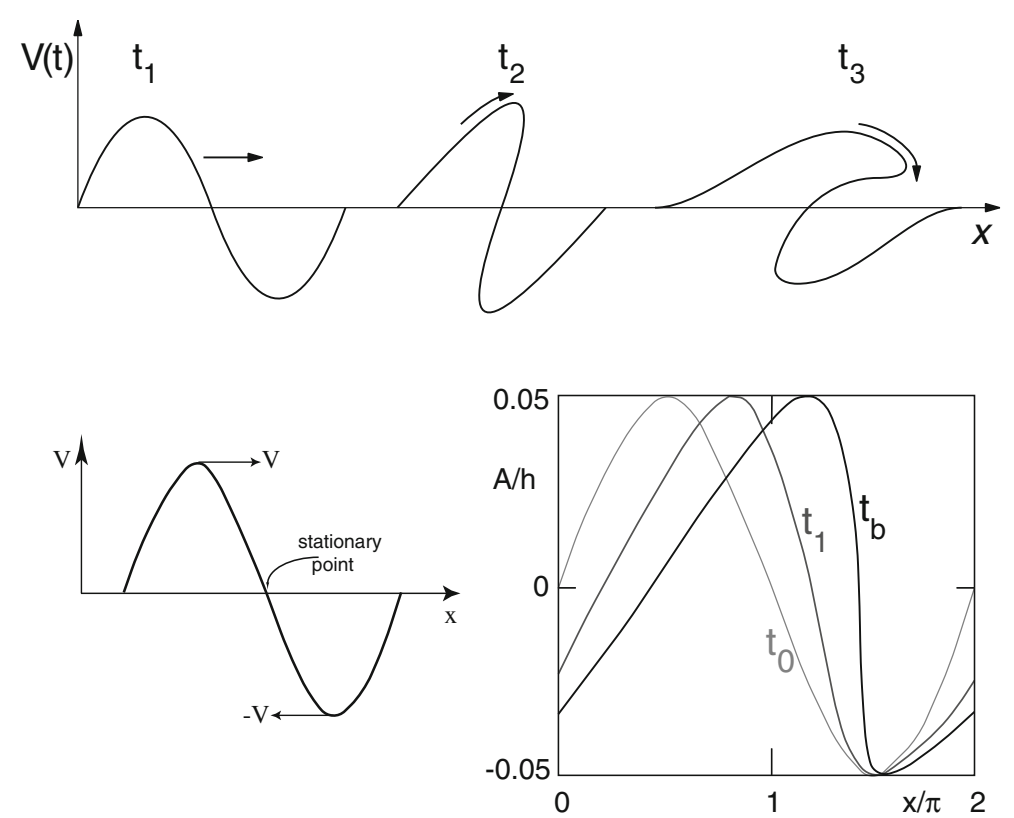

Fig. 5 Top: Schematic of the steeping and wave breaking phenomenon, illustrated for three successive times $t_{1}, t_{2}, t_{3}$. At $t_{2}$ the wave has steepened to maximum, and in $t_{3}$ it collapses in the absence of any retarding effects. Bottom: steeping and final breaking of an initial sinusoidal (simple) wave. Bottom left: Initial wave form in wave frame showing the nonlinear action of the wave on its own shape. Bottom right: Calculation of wave form steeping in a shallow fluid of depth $h$ (after Zahibo et al. 2007). The wave profile is shown at the initial time $t_{0}$, intermediate time $t_{1}$, and breaking time $t_{b}$ when the wave starts turning over. Steeping of the profile is well expressed

\subsubsection{Anomalous dissipation}

Dissipation sets on when the scale of steepness of the wave compares to the dissipation scale $L_{d}$. Then, in the evolution equation of the velocity the next higher order derivatives with respect to $x$ can no longer be neglected, yielding

$$
\mathrm{d} V / \mathrm{d} t=(\partial V / \partial t)+V \nabla_{x} V=\nabla_{x} D \nabla_{x} V-\beta \nabla_{x}^{3} V+\cdots
$$

now with a non-vanishing right-hand side. The first of the new terms is second-order in the gradient $\nabla_{x}$ and corresponds to anomalous diffusion. The second (third-order in $\nabla_{x}$ ) term describes the higher-order dispersion. If diffusion dominates, entropy is produced and steeping ceases, a ramp evolves, and the wave turns into a shock. If dispersion dominates, the shorter wavelength waves run away and a stationary wave packet of finite size is produced which is not a shock, but can, under certain circumstances, also evolve into a shock if the wavelength of the packet shrinks further until it reaches the dissipation scale $L_{d}$. In subcritical shocks this effect ultimately takes place. Hence the question arises, how then is the required anomalous dissipation generated?

The fundamental idea introduced by Sagdeev (1966) is that particles are scattered by the self-excited higher frequency waves and in this way experience anomalous 
collisions which have an effect similar to the real collisions and can cause an anomalous resistivity. Resistivity is defined via the Drude formula $\eta=v / \epsilon_{0} \omega_{p e}^{2}$, with $v=\sigma_{c} N v_{e}$ being the collision frequency. The latter, under collisionless conditions, is the anomalous collision frequency $v_{a}$ and is the quantity containing the interaction between electrons and the nonlinear wave fluctuations. This becomes obvious when realising that the Spitzer-Coulomb collision frequency $v_{\mathrm{C}} \sim \omega_{p e} / N \lambda_{D e}^{3}$ is proportional to the ratio of the plasma wave fluctuation level in thermal equilibrium $W_{t h}=\frac{1}{2} \epsilon_{0}\left\langle\mathbf{e}_{t h}^{2}\right\rangle$ to thermal energy, $v_{\mathrm{C}} \sim \omega_{p e} W_{t h} / N T_{e}$. Under saturated instability conditions it is then reasonable to assume that the actual fluctuations $\left\langle\mathbf{e}^{2}\right\rangle$ replace the thermal fluctuations in this expression which yields the famous Sagdeev formula

$$
v_{a} \simeq\left(W_{\mathrm{sat}} / N T_{e}\right) \omega_{p e}
$$

The problem thus reduces to the determination of the nonlinear saturation level of the unstable wave spectrum. Its determination requires knowledge of the electric current $\mathbf{j} \simeq-e\left\langle N \mathbf{V}_{e}\right\rangle$ as function of the electric wave fluctuation field $\mathbf{e}$.

The evolution of the electron current is - in principle - given by the electronic part of Eq. 14, or Ohm's law (19), if on the right-hand sides the average anomalous electronic friction terms (16) are added. These are the crucial terms containing the waveparticle interactions. The friction term is of the form $-v_{a} N V_{\|}=m_{e} \int \mathrm{d} v^{3} v_{\|} \mathscr{C}_{e}$, and one obtains for the anomalous collision frequency

$$
v_{a} \simeq \frac{1}{N V_{\|}} \nabla_{\|}\left\langle W_{\text {sat }}\right\rangle, \quad \nabla_{\|} W_{k} \simeq \frac{2 \gamma\left(\omega, \mathbf{k}, W_{k}\right)}{\left|V_{\|}-\partial \omega / \partial k_{\|}\right|} W_{k}
$$

where the average wave power is $\left\langle W_{\text {sat }}\right\rangle=\frac{1}{2} \epsilon_{0}\left\langle\left|\partial_{\omega}(\omega \epsilon) \mathbf{e}^{2}\right|\right\rangle$, and $W_{k}$ its Fouriertransformed (which is the wave spectral energy density). $\omega(\mathbf{k})$ is the frequency of the unstable wave, and the wave spectral density $W_{k}$ evolves according to the second equation in (41). Here $\epsilon(\omega, \mathbf{k})$ is the dielectric dispersion function which contains the dispersion of the wave, and $\gamma(\omega, \mathbf{k})$ is the growth rate of the instability. ${ }^{14}$ Usually the current-drift speed $\left|V_{\|}\right| \gg\left|\partial \omega / \partial k_{\|}\right|$is much larger than the wave group velocity, and the latter can be neglected. This yields the inverse square dependence of $v_{a} \propto\left|V_{\|}\right|^{-2}$ of the anomalous collision frequency on the current drift velocity.

The dissipation depends crucially on the particular wave spectrum $W_{k}$. There are a large number of possibilities of which kind of waves are excited by the electric current in the shock front. In subcritical shocks, identification of the waves is necessary, while in supercritical shocks it is not believed to be of primary importance for the shock dynamics. There, wave type may play a role in the generation of high energy particles and radiation. Candidates for the unstable waves are the two-stream instability which becomes unstable at current drifts $V_{\|}>v_{e}$, i.e. at very high current speeds, and the ion-acoustic instability which is unstable at $v_{e}>V_{\|}>c_{\mathrm{ia}}$, when the current drift speed exceeds the ion acoustic velocity $c_{\text {ia }}$, the latter causing an anomalous collision

\footnotetext{
14 For the determination of $\epsilon(\omega, \mathbf{k})$, wave frequency $\omega(\mathbf{k})$ and linear growth rate $\gamma(\omega, \mathbf{k})$ the reader is referred to any textbook on basic plasma physics (e.g. Baumjohann and Treumann 1996).
} 
frequency $v_{a} \simeq 0.01\left(V_{\|} / c_{\mathrm{ia}}\right)\left(T_{e} / T_{i}\right) \omega_{p i}$, being favoured by high electron temperatures $T_{e} \gg T_{i}$. Since the required velocities are high, it remains unclear whether these waves contribute to subcritical shocks.

Probably, in subcritical shocks the two most important wave modes and their corresponding instabilities are the lower-hybrid-drift instability and the modified-twostream instability. The former arises when the scale of width of the shock front is comparable to the ion inertial length $\Delta_{\mathrm{sh}} \sim \lambda_{i}$. The latter can be excited when the currents flow perpendicular to the magnetic field, as is the case for the electron current in the shock ramp. Both instabilities have much lower thresholds than the two-stream and ion-acoustic instabilities. They yield comparably large anomalous collision frequencies $v_{a} \sim \omega_{\mathrm{lh}}$ of the order of the lower-hybrid frequency $\omega_{\mathrm{lh}} \simeq \sqrt{m_{e} / m_{i}} \omega_{c e}$, being candidates for the required dissipation in subcritical shocks. The problem about the long favoured lower-hybrid instability is that it is readily damped in a high- $\beta$ plasma that is typical for a hot shock ramp. Thus the modified two-stream instability (MTSI) remains as the most promising candidate. It, however, requires the presence of a shock-normal electric field across the shock ramp transition on a scale $\lesssim \lambda_{i}$ in order to keep the ions non-magnetic. Fortunately, in contrast to generating a very steep density gradient, production of a cross-shock electric potential drop is quite natural in any magnetised shock formation. Even though this field is too weak to enable ion reflection, it serves to accelerate electrons across the shock in an ambipolar manner, thereby reducing the scale below the ion inertial length.

The magnetised electrons perform an $\mathbf{E} \times \mathbf{B}$-drift in the shock magnetic and crossshock electric fields. The direction of this drift is perpendicular to the magnetic field and tangential to the shock. It produces an electron-drift current

$$
\mathbf{j}_{d}=-\frac{e N}{B^{2}} \mathbf{E} \times \mathbf{B}
$$

that flows along the shock, most probably in the shock ramp, and whose magnetic field contributes to the steeping of the magnetic shock ramp and the first shock-magnetic overshoot. Its magnitude in terms of the upstream Mach number $\mathscr{M}_{A}$ and the cross-shock potential $\bar{U}(x)$ has been given in Eq. 34. This drift current stores free energy, which it provides to the excitation of the modified-two-stream instability at wavelengths $\lambda \gg \lambda_{\mathrm{D}}$. The growth rate of the modified-two-stream instability is of the order of roughly ten times the ion cyclotron frequency. The MTSI does not exist for strictly parallel and strictly perpendicular shocks, however, but this is not a severe restriction, for these two marginal cases will rarely be realised in practice. It is excited in the shock transition on transverse scales shorter than the ion-inertial length and on tangential scales of many wavelengths along the shock surface.

\subsection{Subcritical shock model}

\subsubsection{Cold plasma model}

Following Sagdeev (1966) we refer to a one-dimensional cold $\left(\beta_{1} \ll 1\right)$ two fluid damped oscillator model, where the shock is a magnetosonic wave that is described by 
the continuity and momentum conservation equations. The latter reduce to ion pressure balance and stationary electromagnetic conditions. Before transforming them, reducing them to one single equation for $b(x) \equiv B_{z}(x) / B_{1}$, the ratio of the magnetic field to the upstream magnetic field, these equations read

$$
\begin{gathered}
\nabla_{x} B_{z}=\mu_{0} e N v_{y}, \quad \nabla_{x} E_{y}=0, \quad \nabla_{x} N v_{x}=0 \\
\nabla_{x}\left(\frac{1}{2} m_{i} N v_{x}^{2}+\frac{B_{z}^{2}}{2 \mu_{0}}\right)=0, \quad m_{e} N v_{x} \nabla_{x} v_{y}=-e N E_{y}+e N v_{x} B_{z}-v_{\mathrm{an}} m_{e} N v_{y}
\end{gathered}
$$

We now define $\xi=x / \lambda_{e}$ and ${ }^{\prime} \equiv \mathrm{d} / \mathrm{d} \xi$. Then, after transformation to the shock velocity frame $V_{\text {sh }}$, one obtains

$$
b^{\prime \prime}=b-1+A b\left(1-b^{2}\right)-a b^{\prime}
$$

with $A \equiv B_{1}^{2} / 2 \mu_{0} N m_{i} V_{\text {sh }}^{2}$, and $a \equiv v \lambda_{e} / V_{\text {sh }}$. This is the equation of motion of a hypothetical particle with coordinate $b$ and time $\xi$, including frictional dissipation. In the absence of dissipation one defines the Sagdeev pseudo-potential $S(b)$

$$
2 S(b)=(b-1)^{2}\left[A(b+1)^{2}-2\right], \quad b<b_{\max }=\left(2 V_{\mathrm{sh}} / V_{A 1}\right)-1
$$

Negative values of $S(b)$ constitute a potential trough for the hypothetical particle if the field amplitudes $b<b_{\max }$ are smaller than a maximum value $b_{\max }$. For a given $S(b)$ the hypothetical particle performs a stationary oscillation in this potential trough with amplitude equal to the distance between the walls. The shape of the trough is shown as the heavy line marked $s=0$ on the right in Fig. 6 for the special case $\mathscr{M}_{A}=1.6$. The maximum possible amplitude is reached for $S(b)=0$. The ratio $V_{\mathrm{sh}} / V_{A 1}=\mathscr{M}_{\mathrm{sh}}$ is the shock-Mach number, i.e. the Mach number of the possible stationary solutions in this dissipationless case. Including the resistive damping, this amplitude decreases during the oscillation and the pseudo-particle will ultimately settle in the final state at the minimum of the pseudo-potential $S(b)$. The potential minimum is at the stationary downstream value of the normalised magnetic field

$$
2 b\left(S_{\min }\right) \equiv 2 b_{2}=\left(1+8 \mathscr{M}_{\mathrm{sh}}^{2}\right)^{\frac{1}{2}}-1
$$

This corresponds to the final stationary shock state of a subcritical fast magnetosonic shock. It is independent of the dissipation even though it has been reached only due to the action of the anomalous dissipation $a$. A shock of this kind is a weak shock since the plasma pressure contribution has been neglected and the plasma has been assumed to be cold, such that the heating of the plasma by the shock itself is also small and does not appear anywhere. The shock profile can be determined from the solution of the equation for $b$. It is found to be a spatial oscillation reaching maximum at the shock ramp and decreasing exponentially behind the shock with spatially damped amplitude $b(\xi) \sim \exp (-a \xi) \sin \left(\xi \sqrt{\mathscr{M}_{\mathrm{sh}}-1}\right)($ see, e.g., Tidman and Krall 1971). Because of the simplifying assumptions this solution holds only for small shock amplitudes. 

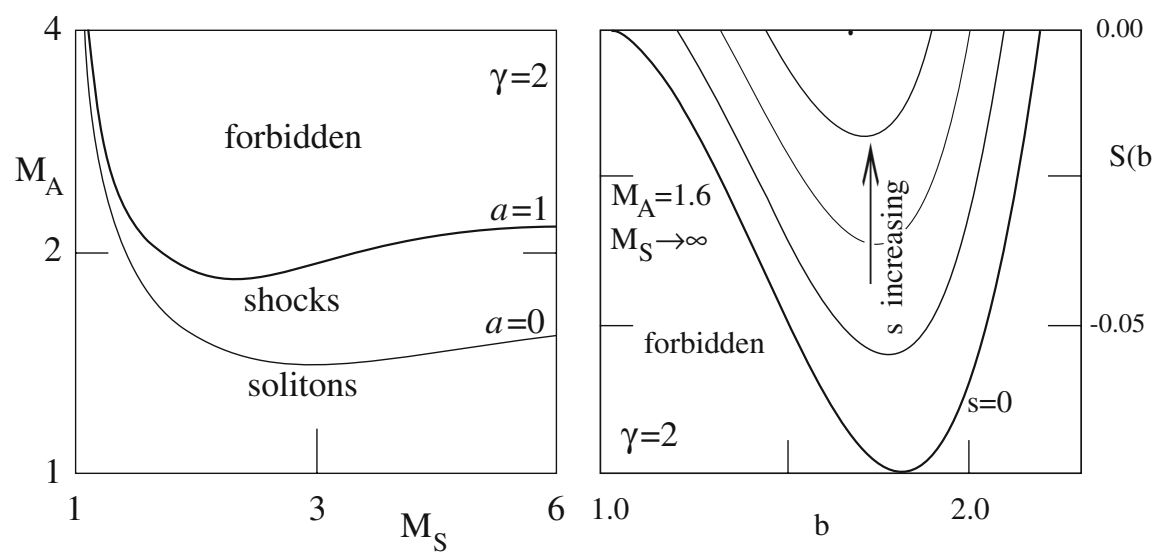

Fig. 6 Left: The allowed regions in $\left(\mathscr{M}_{A}, \mathscr{M}_{S}\right)$-space for solitons and shocks depending on the resistive dissipation coefficient $a$ for a given adiabatic index $\gamma=2$. Subcritical shocks exist only in the narrow domain between the two curves for $0<a<1$. Right: The shape of the Sagdeev pseudo-potential $S(b)$ for $\mathscr{M}_{A}=1.6, \mathscr{M}_{s} \rightarrow \infty$ and $\gamma=2$ as function of the entropy density $s$. For $s=0$, corresponding to $a=0$, the Sagdeev pseudo-potential has its largest excursion into the negative domain. For this case, solitons exist throughout the entire region inside the curve. With increasing $s$ the domain shrinks, and no soliton solutions exist anymore being replaced by shock solutions. A shock starts at one of those curves and wanders upward in the diagram until it reaches the maximum entropy point on the $S=0$ axis (black dot) (data taken from Ziegler and Schindler 1988)

\subsubsection{Extension to warm plasma}

The zero temperature, small amplitude case of subcritical perpendicular shocks can be analytically extended to subcritical shocks of arbitrary amplitudes in a warm two-component plasma consisting of electrons and ions (Ziegler and Schindler 1988). In the warm case we measure the length along $x$ in $\bar{\lambda}=\sqrt{\lambda_{e} \lambda_{i}}$ finding $b v_{x}-1-\frac{a}{v_{x}} b^{\prime}=b^{\prime \prime}$, an equation that still contains the flow velocity $v_{x}$. Not neglecting the thermal pressure, one must include the equation of energy conservation, with entropy density $s$. This leads to the desired equation for $v_{x}$, which is more complicated (Biskamp 1973; Ziegler and Schindler 1988). It includes both, the upstream Alfvén and the sound wave Mach numbers $\mathscr{M}_{A}, \mathscr{M}_{s}$. The latter is due to the finite thermal pressure. Again, the resistive Joule dissipation is contained in the coefficient $a=v_{\mathrm{an}} m N / \bar{\lambda}$, with $m=m_{i}+m_{e}$. Its normalised form has been given by Biskamp (1973). At high Mach numbers there are two solutions for the velocity indicating the absence of a continuous solution that is independent of resistivity. This is in agreement with the earlier claim that high Mach number shocks are supercritical and require additional dissipation. The small amplitude Sagdeev solution follows for $\mathscr{M}_{s} \rightarrow \infty$, negligible plasma pressure $\mathrm{P} / B_{1}^{2}\left(b^{2}-1\right) \ll 1$, and $v_{x} \rightarrow V_{\text {sh }}$, in which case $s(\bar{x})$ can be neglected.

In the more general case, real stationary solutions that connect the homogeneous states at $x= \pm \infty$ exist only in a limited Mach number range for flows that are supersonic with respect to the phase velocity of linear waves propagating perpendicular to the magnetic field $\mathbf{B}$. The regions of existence of these solutions are given on the left in Fig. 6. The curves in this figure separate the forbidden and allowed regimes in the 

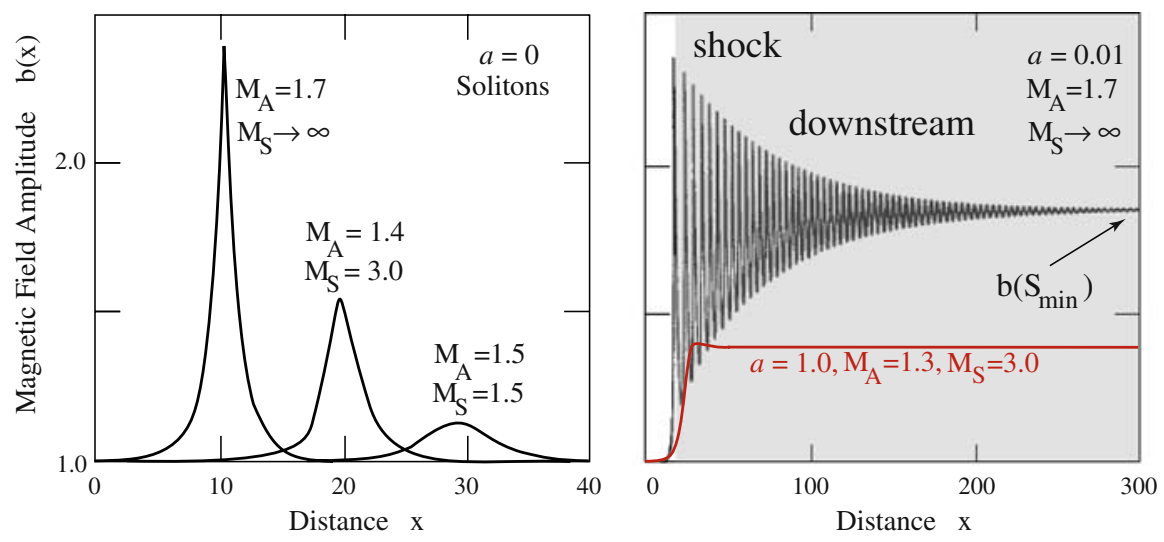

Fig. 7 Left: Numerical calculation of soliton forms for $a=0$ and different Mach number combinations. Right: The shape of the subcritical shock in the two-fluid model for finite dissipation $a=0.01$. The solution has a steep ramp and a short wavelength downstream oscillation which damps slowly out with normalised distance from the shock ramp until it reaches the final amplitude $b\left(S_{\min }\right)$ corresponding to the minimum of the Sagdeev pseudo-potential for the given combination of Mach numbers. The red line shows the asymptotic shock profile for maximum dissipation $a=1$. In this case the shock does not exhibit oscillations but is a smooth ramp (data taken from Ziegler and Schindler 1988)

$\left(\mathscr{M}_{A}, \mathscr{M}_{S}\right)$-plane. Solutions exist only below these curves depending on dissipation $a$. In the absence of dissipation, solitons exist below the curve $a=0$. With increasing dissipation, up to its maximum value $a=1$, no soliton solutions exist; this is the range of subcritical shock waves. They are confined between the curves $a=0$ and $a=1$. On the right-hand side of this figure the parts of the Sagdeev pseudo-potential curves $S(b)<0$ that correspond to real solutions are given as functions of entropy density $s$ that is generated by the anomalous dissipation. It is seen that the domain of shock solutions shrinks gradually with increasing dissipation.

Figure 7 shows the results obtained by numerically integrating the fully dissipative magnetosonic two-fluid warm-plasma equations. The left part of the figure shows the dissipationless case $a=0$ for different combinations of Mach numbers $\mathscr{M}_{A}, \mathscr{M}_{s}$. Magnetosonic solitons of different forms and amplitudes are obtained. The equations permit for multi-soliton solutions. Here only one soliton of each kind is shown.

The black curve on the right of the figure is a typical magnetosonic shock solution with weak dissipation $a=0.01$ and negligible thermal pressure. The solution in this special case is a steep shock front with large overshoot, corresponding to the steep leading edge of the first magnetosonic soliton on the left. The dissipation does not allow for the soliton amplitude to return to its initial upstream level because the steep gradient scale in the shock front approaches the dissipation length-scale such that dissipation becomes important and generates entropy. This causes the oscillation downstream of the shock to be spatially damped. The oscillation amplitude decreases exponentially with distance from the shock as predicted by simplified small amplitude theory (Sagdeev 1966) until the field amplitude settles at $b\left(S_{\min }\right)$ far behind the shock transition.

Also shown in this figure is the case of maximum dissipation $a=1$ when the shock transition scale is large. Under these maximum-dissipation conditions, the shock does 
not exhibit downstream oscillations but becomes a steep and smooth shock ramp with small magnetic overshoot and laminar downstream state corresponding to a completely dissipation-dominated Burgers shock transition. Again the amplitude of the shock corresponds to $b\left(S_{\min }\right)$ for the chosen combination of Mach numbers, yielding a smaller final downstream amplitude than in the oscillatory case. The downstream state is completely laminar with no oscillations evolving for these marginal conditions. Of course, the solutions are strictly independent of time such that no turbulence can evolve. Under real conditions allowing for time variations, the stationarity will become violated.

Kennel and Sagdeev (1967) developed a subcritical shock model that is a combination of the steeping of an ion-acoustic wave and the excitation of an Alfvén wave which dispersively evolves into a magnetic shock ramp. The idea is that in a warm plasma stream conditions are given under which the ion-acoustic mode can be excited along the magnetic field to large amplitude, thereby producing a pressure anisotropy which can excite the firehose instability. When working in tandem, both effects would generate a subcritical shock with a magnetic Alfvén ramp and anomalous resistivity provided by the ion-acoustic instability. Kennel and Sagdeev (1967) also proposed another variant of this model in which the coupling of the ion-sound wave and the Alfvén wave was not required as the entire process of steeping was attributed to a magnetoacoustic wave. The generation of anomalous resistivity was left to the action of ion acoustic waves excited in the shock. However, both models have later been replaced by the realisation that whistlers are more important in shock generation. Still, the problem of generation of anomalous dissipation has not yet been solved.

\subsection{Conclusions}

Based on the considerations in this section we may complete the scenario of evolution of a subcritical shock. The subcritical shock is the result of the nonlinear steeping of a magnetosonic (whistler) wave. Dispersive effects may or may not lead to the presence of upstream phase locked whistlers in front of it. This depends on the shape of the dispersion curve $\omega(\mathbf{k})$ in the $(\omega, \mathbf{k})$-plane. If, as is the case for electron whistlers, the dispersion curve is convex from below, then the short wavelength sidebands run out of the shock in the upstream direction but cannot escape from the shock because the supermagnetosonic inflow from upstream convects them back towards the shock. Thus, in equilibrium they build up a phase-locked standing whistler-precursor train in front of the shock with amplitude decreasing away from the shock in the upstream direction. For concave dispersion (as is the case for the magnetosonic mode) the short wavelength sidebands are slower than the shock and form an oscillating downstream wave trail that contributes to the downstream turbulence and whose amplitude decreases spatially with distance away from the shock transition.

During wave steeping a solitary wave forms with ramp steepness on the scale of (or larger than) the ion inertial length $\Delta_{\mathrm{sh}}<\lambda_{i}$. When the upstream particles hit the soliton ramp, the difference in the ion and electron gyroradii produces a cross-shock potential $\bar{U}$. This potential is not strong enough for reflecting the ions, but it causes a cross-field drift of the electrons which drives an electron current in the shock ramp 
along the shock. Even when this current is weak, it excites the MTSI. When the MTS waves quasilinearly saturate, anomalous collisions scatter the electrons and generate dissipation, Joule heating of electrons and ions, and the entropy required for the transformation of the soliton into a shock. The anomalous collisions cause the electrons to diffuse ambipolarly across the shock to shorten the length scale below the ion inertial length, thus keeping the ions non-magnetic and the electron drift scale short enough for the MTSI to be maintained.

As a side effect in this scenario, we note that the MTSI also evolves into a series of localised electric field structures along the magnetic field, so-called electron holes, the effect of which is to accelerate some electrons into beams along the magnetic field. These may cause some radiation at the local plasma frequency $\omega_{p e}$ that escapes from the shock into free space which, in principle, can be observed remotely. Since subcritical shocks have very low Mach numbers $\mathscr{M}_{c}<2.7$ one expects that they contribute to turbulence in media only where the velocity differences between interacting streams exceed the magnetosonic speed by a small amount, in which case subcritical shocks do not show up as genuine shocks.

Under astrophysical conditions one expects that subcritical shocks contribute to internal shocks in a turbulent flow, i.e., their main effect is to contribute to turbulence. On the other hand, heliospheric experience also suggests that they are generated when a very high-Mach number flow interacts with a dense surrounding medium, such as an unmagnetised stellar atmosphere or a very weakly magnetised interstellar or intergalactic plasma. In this case the high-Mach number flow becomes heavily mass loaded and slows down to speeds that exceed the magnetosonic speed by only a small amount, conditions typical for subcritical shock generation. This case is probably realised in the solar wind behind the termination shock where the interstellar medium serves as both an obstacle and a dense gas source which mass-loads the solar wind plasma. Presumably, the resulting heliospheric bow shock wave in the mass-loaded medium is such a mass-loaded subcritical shock. Whether or not such a claim holds has to await the passage of the Voyager spacecraft after several decades. Similar conditions might exist in the lobes of jets where they resolve into the surrounding medium.

In the following sections we describe the astrophysically more important class of shocks, the various types of supercritical shocks.

\section{Quasi-perpendicular supercritical shocks}

\subsection{Reflected particle dynamics}

We speak of quasi-perpendicular super-critical shocks when the shock normal angles $\theta_{B n}<45^{\circ}$, for good reasons. First, super-critical shocks cannot be maintained by dissipation alone. This has been clarified in Sects. 1 and 2.1. The inflow of matter into a supercritical shock is so fast that the time scales on which dissipation would take place are too long for dissipating the excess energy and lowering the inflow velocity below the downstream magnetosonic velocity. Hence, the condition for criticality, as we have shown in Sect. 1.3, is that the downstream flow velocity becomes equal to 
the downstream magnetosonic speed, which yielded the critical Mach number (29), $\mathscr{M}_{c} \lesssim 2.76$. We have also shown that $\mathscr{M}_{c}\left(\theta_{B n}\right)$ is a function of the shock normal angle and can become quite small, even though of course $\mathscr{M}\left(\theta_{B n}\right) \gtrsim 1$ for existence of a shock.

In order to help maintain a shock in the supercritical case, the shock must inhibit an increasing number of ions from passing across its ramp. This is achieved by reflecting the excess particles back upstream. Particle reflection is not a direct dissipation process, however. Rather, it is an emergency act of the shock, which throws a fraction of the incoming ions back upstream and thus reduces both the inflow momentum and energy density. Clearly, this reflection process slows the shock down by attributing a negative momentum to the shock itself. The shock slips back, and thus in the shock frame also reduces the difference velocity to the inflow, i.e. it reduces the Mach number. In addition, however, the reflected ions form an unexpected obstacle for the inflow and in this way reduce the Mach number by another amount.

These processes are very difficult to treat and understand. We will go into more detail here. However, we must first ask, what is the reason for the rigid limit in $\theta_{B n}$ that allows calling a shock a quasi-perpendicular supercritical shock. ${ }^{15}$ The answer is that a shock belongs to the class of quasi-perpendicular shocks as long as the reflected particles remain confined to the vicinity of the shock, being unable to escape from it upstream along the upstream magnetic field. After having performed half a gyro-circle back upstream, they will thus necessarily return to the shock ramp and ultimately traverse it. At this time, however, they have contributed to the slow-down of the upstream flow. They have also been accelerated sufficiently and increased their gyro radius until they drop out of the bulk plasma flow and traverse the shock ramp like independent test particles. In this way they have cheated the shock. After having passed the shock ramp they mix into the downstream flow and contribute to downstream wave excitation and heating.

In order to appreciate this we return to the orbit a particle performs in interaction with a supercritical shock when it is reflected from the shock. In the simplest possible model one assumes the shock to be a plane surface, and the reflection, being specular, turning the component $v_{n}$ of the instantaneous particle velocity $\mathbf{v}$ normal to the shock by $180^{\circ}$, i.e. simply inflecting it. Here we follow the explicit calculation for the idealized conditions as given by Schwartz et al. (1983) who treated the problem in a little more general way. One should, however, keep in mind that the assumption of ideal specular reflection is the extreme limit of what happens in reality. In fact, reflection may by no means be specular for many reasons. One reason is that the shock

\footnotetext{
15 The theory given in this section is purely kinematic. It represents only the marginal state of ideal specular reflection of a charged particle from an infinitely thin shock front. We have not justified the assumption of specular reflection. Reflection under real conditions will by no means be specular. The shock has a finite width, and any particle that arrives at the shock penetrates to a different depth into the shock. Once the particle is reflected, it will be reflected from some point inside the shock and may or may not return upstream. The mechanism of reflection depends on the interaction between the particles that arrive at the shock, the shock electric field, and the wave spectrum inside the shock. All these are only vaguely known and can be determined only from self-consistent considerations. Nevertheless, the assumption of specular reflection has turned out to be a surprisingly good approximation when classifying shocks with respect to their quasi-perpendicular and quasi-parallel properties.
} 
ramp is not a rigid wall; the particles penetrate into it over a distance of a fraction of their gyroradius, they encounter the compressed plasma and magnetic field, experience the electric field in the shock ramp (the turbulent wave spectrum), they interact with the waves and excite waves during this interaction and during their approach to the shock. Altogether, it must be stressed that the very mechanism of reflection is, indeed, poorly known - specular reflection is no more than a convenient assumption. Nevertheless, observations suggest that assuming specular reflection seems to be quite a good approximation to reality.

Figure 9 shows the coordinate frame used at the planar shock, with shock normal $\mathbf{n}$, magnetic and velocity unit vectors $\hat{b}, \hat{v}$, respectively. Shown are the angles $\theta_{B n}, \theta_{V n}, \theta_{B V}$. The velocity vector $\mathbf{V}_{\mathrm{HT}}$ is the de Hoffmann-Teller velocity which lies in the shock plane and is defined in such a way that in the coordinate system moving along the shock plane with velocity $\mathbf{V}_{\mathrm{HT}}$, the plasma flow is along the magnetic field (Fig. 8); $\mathbf{V}-\mathbf{V}_{\mathrm{HT}}=-v_{\|} \hat{b}$. The guiding centres of the particles in this frame move all along the magnetic field. For this reason it is convenient to consider the motion of particles in the de Hoffmann-Teller frame. Hence, the velocity vector has the two components

$$
\begin{aligned}
v_{\|} & =V\left(\cos \theta_{V n} / \cos \theta_{B n}\right), \\
\mathbf{V}_{\mathrm{HT}} & =V\left[-\hat{v}+\hat{b} \cos \theta_{V n} / \cos \theta_{B n}\right] \equiv(\mathbf{n} \times \mathbf{V} \times \mathbf{B}) / \mathbf{n} \cdot \mathbf{B}
\end{aligned}
$$

Because of the continuity of $B_{n}$ and the tangential electric field, the de HoffmannTeller velocity is the same on both sides of the shock ramp,. There is no induction electric field $\mathbf{E}=-\mathbf{n} \times \mathbf{V} \times \mathbf{B}$. The remaining problem is two-dimensional (see the coplanarity theorem which holds strictly in this purely kinematic case). The particle velocity is described by the motion along $\hat{b}$ plus the gyromotion in the plane perpendicular to $\hat{b}$ :

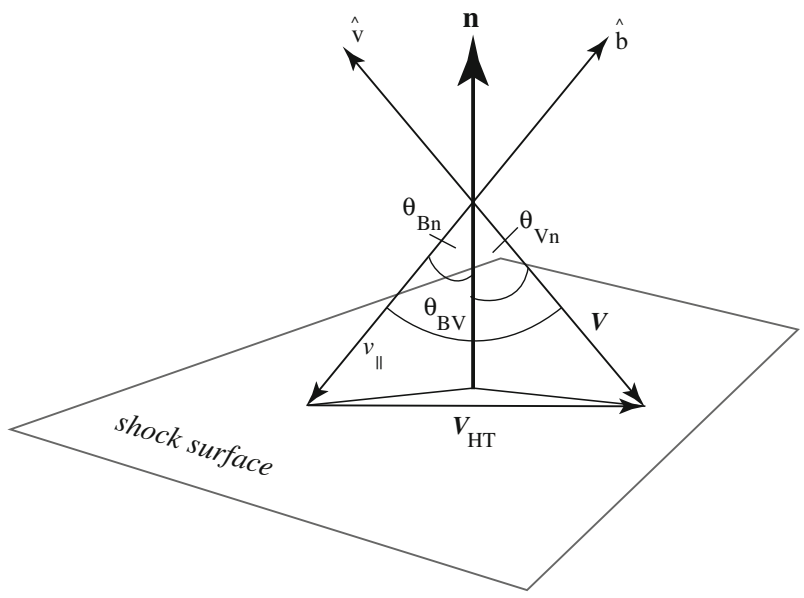

Fig. 8 The shock coordinate system showing the shock normal $\mathbf{n}$, velocity and magnetic field directions $\hat{v}, \hat{b}$, the three angles $\theta_{B n}, \theta_{V n}, \theta_{B V}$ between $\hat{b}$ and $\mathbf{n}$, velocity $\mathbf{V}$ and $\mathbf{n}$, and velocity $\mathbf{V}$ and $\hat{b}$, respectively. The velocity $\mathbf{V}_{\mathrm{HT}}$ in the shock plane is the de Hoffmann-Teller velocity 


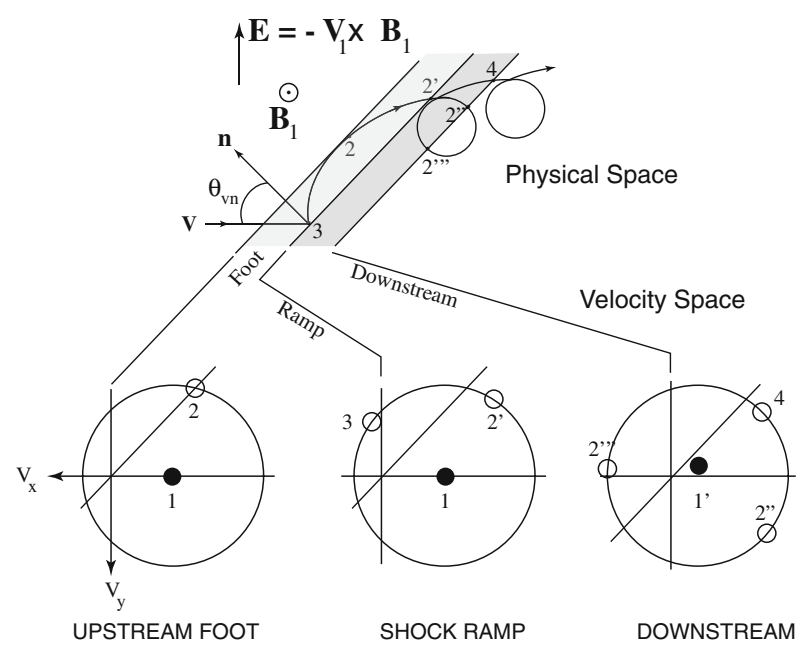

Fig. 9 Top: Reflected ion orbits in the foot of a quasi-perpendicular shock in real space. The ion impacts under an instantaneous angle $\theta_{v n}$, is reflected from the infinitely thin shock, performs a further partial gyration in the upstream field $\mathbf{B}_{1}$ where it is exposed to the upstream convection electric field $\mathbf{E}=-\mathbf{V}_{1} \times \mathbf{B}_{1}$ in which it is accelerated as is seen from the non-circular section of its orbit in the shock foot. It hits the shock ramp a second time now at energy high enough to overcome the shock potential, passing the ramp and arriving in the compressed downstream magnetic field behind the shock where it performs gyrations of reduced gyro-radius. Bottom: The ion distribution function mapped into velocity space $v_{x}, v_{y}$ for the indicated regions in real space, upstream in the foot, at the ramp, and downstream of the shock ramp. Upstream, the distribution consists of the incoming dense plasma flow (population 1, dark circle at $v_{y}=0$ ) and the reflected distribution 2 at large negative $v_{y}$. At the ramp in addition to the incoming flow 1 and the accelerated distribution 2' there is the newly reflected distribution 3. Behind the ramp in the downstream region the inflow is decelerated 1' and slightly deflected toward non-zero $v_{y}$, and the energized passing ions exhibit gyration motions in different instantaneous phases, two of them (2", 4) directed downstream, one of them (2"') directed upstream. (after Sckopke et al. 1983, courtesy American Geophysical Union)

$$
\mathbf{v}^{\prime}(t)=v_{\|}^{\prime} \hat{b}+v_{\perp}\left[\hat{x} \cos \left(\omega_{c i} t+\phi_{0}\right) \mp \hat{y} \sin \left(\omega_{c i} t+\phi_{0}\right)\right]
$$

The unit vectors $\hat{x}, \hat{y}$ point along the orthogonal coordinates in the gyration plane of the ion, the phase $\phi_{0}$ accounts for the initial gyro-phase of the ion, and \pm accounts for the direction of the upstream magnetic field being parallel (+) or antiparallel to $\hat{b}$. In specular reflection the velocity component along $\mathbf{n}$ is reversed, and (for cold ions) becomes $\mathbf{v}^{\prime}=-v_{\|} \hat{b}+2 v_{\|} \cos \theta_{B n} \hat{n}$, which (with $\phi_{0}=0$ ) yields

$$
\begin{aligned}
v_{\|}^{\prime} / V & =\left[\cos \theta_{V n} / \cos \theta_{B n}\right]\left(2 \cos ^{2} \theta_{B n}-1\right) \quad \text { and } \\
v_{\perp} / V & =2 \sin \theta_{B n} \cos \theta_{V n}
\end{aligned}
$$

A reflected particle returns to the shock when the upstream component of the velocity $v_{x}=0$, which for $\phi_{0}=0$ yields

$$
\mathbf{x}^{\prime}(t)=v_{\|}^{\prime} t \hat{b}+\left(v_{\perp} / \omega_{c i}\right)\left\{\left(\sin \omega_{c i} t\right) \hat{x} \pm\left(\cos \omega_{c i} t-1\right) \hat{y}\right\}
$$


After scalar multiplication of this expression with $\mathbf{n}$, the ion displacement normal to the shock in upstream direction becomes

$$
\mathbf{x}_{n}^{\prime}\left(t^{*}\right)=v_{\|}^{\prime} t^{*} \cos \theta_{B n}+\left(v_{\perp} / \omega_{c i}\right) \sin \theta_{B n} \sin \omega_{c i} t^{*}=0
$$

It vanishes at time $t^{*}$ when the ion re-encounters the shock with normal velocity $v_{n}\left(t^{*}\right)=v_{\|}^{\prime} \cos \theta_{B n}+v_{\perp} \sin \theta_{B n} \cos \omega_{c i} t^{*}$. Setting this to zero, one obtains for the maximum displacement time

$$
\omega_{c i} t_{m}=\cos ^{-1}\left[\left(1-2 \cos ^{2} \theta_{B n}\right) 2 \sin ^{2} \theta_{B n}\right]
$$

which must be inserted in $\mathbf{x}_{n}$ yielding for the distance a reflected ion with gyro-radius $r_{c i}=V / \omega_{c i}$ can reach in the upstream direction

$$
\Delta x_{n}=r_{c i} \cos \theta_{V n}\left[\omega_{c i} t_{m}\left(2 \cos ^{2} \theta_{B n}-1\right)+2 \sin ^{2} \theta_{B n} \sin \omega_{c i} t_{m}\right]
$$

This distance, for a perpendicular shock $\theta_{B n}=90^{\circ}$, is

$$
\Delta x_{n} \simeq 0.7 r_{c i} \cos \theta_{V n}
$$

which is less than an ion gyro radius. Note that this distance depends strongly on the angle the velocity makes with the normal of the shock, and on the shock normal angle.

The important conclusion is drawn from consideration of the argument of $\cos ^{-1}$ in the expression (52) for $\omega_{c i} t_{m}$ which exceeds unity for $\theta_{B n} \leq 45^{\circ}$. Hence, there are no solutions for such angles. Reflected ions return to the shock only when the magnetic field makes an angle with the shock normal larger $\theta_{B n}>45^{\circ}$. For less inclined shock normal angles, the reflected ions escape along the magnetic field upstream of the shock and do not return.

This sharp distinction between shock normal angles $\theta_{B n}<45^{\circ}$ and $\theta_{B n}>45^{\circ}$ thus provides the clear natural discrimination between quasi-perpendicular and quasi-parallel shocks we were looking for. Of course, this distinction holds only under the simplifying assumption, made earlier, of specular reflection.

\subsubsection{Foot formation and ion acceleration}

Shock reflected ions in a quasi-perpendicular shock cannot escape far upstream. Their penetration into the upstream plasma is severely restricted by formula (53). Within this distance the ions perform a gyrational orbit before returning to the shock.

Since the reflected ions are about at rest with respect to the inflowing plasma, they are sensitive to the inductive convection electric field $\mathbf{E}=-\mathbf{V}_{1} \times \mathbf{B}_{1}$ which is along the shock surface both perpendicular to the flow and to the magnetic field. The reflected ions behave very similar to pick-up ions. In the electric field they become accelerated 
in the direction of $\mathbf{E}$ and achieve higher energy (Schwartz et al. 1983). When returning to the shock their maximum (minimum) achievable energy is

$$
\mathscr{E}_{\max }=\frac{1}{2} m_{i}\left[\left(v_{\|}^{\prime}+V_{\mathrm{HT} \|}\right)^{2}+\left(V_{\mathrm{HT} \perp} \pm v_{\perp}\right)^{2}\right]
$$

The maximum energy is larger than their initial energy when they have initially met the shock ramp. Under favorable conditions, they might now overcome the shock ramp potential and escape downstream. Otherwise, when becoming reflected again, they gain energy in a second round until they have picked up sufficient energy for passing the shock.

In addition to this energization of reflected ions, which in the first encounter have not made it across the shock, the reflected ions, when gyrating upstream and being accelerated in the convection electric field, constitute a current layer just in front of the shock ramp of current density $j_{y} \sim e N_{i, \text { refl }} v_{y}$,refl. This gives rise to a foot magnetic field of magnitude $B_{z \text {, foot }} \sim \mu_{0} j_{y} \Delta x_{n}$, where $x_{n}$ is the width of the foot region (in normal direction). The foot current is essentially a line current. It increases the upstream magnetic field and flattens the upward part of the ramp magnetic field. These effects together produce a flat foot and a ramp that is steeper than it would be in the absence of the foot. In addition, the foot magnetic field forms a barrier for the incoming flow

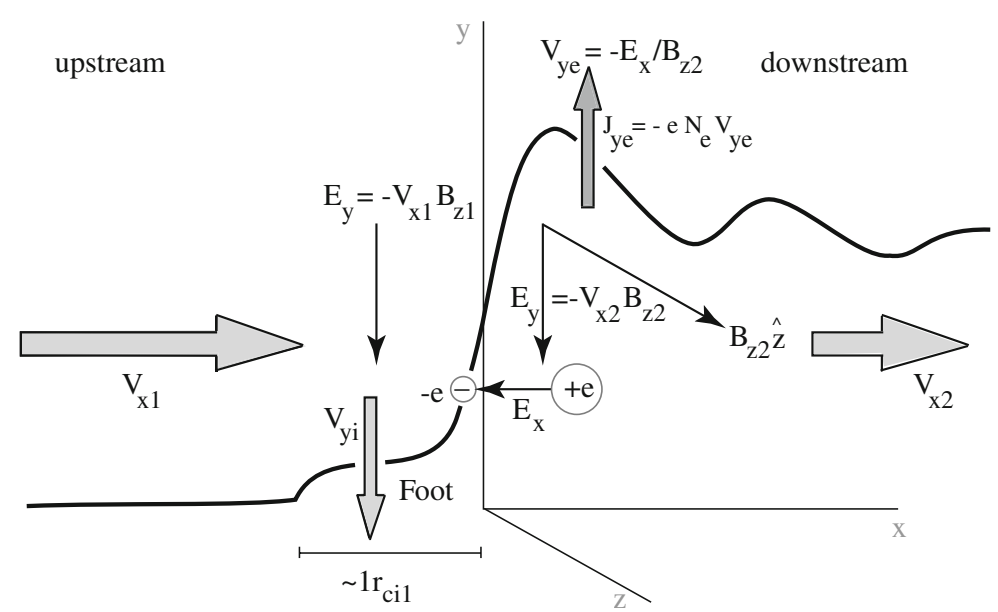

Fig. 10 Geometry of an ideally perpendicular supercritical shock showing the field structure and sources of free energy. The compressive profile of the shock stands for the compressed profile of the magnetic field $|\mathbf{B}|$, density $N$, temperature $T$, and pressure $N T$. The inflow of velocity $V_{1}$ and outflow of velocity $V_{2}$ is in $x$ direction, and the magnetic field is in $z$ direction. Charge separation over an ion gyroradius $r_{c i}$ in the shock ramp magnetic field generates a charge separation electric field $E_{x}$ along the shock normal which reflects the low-energy ions back upstream. These ions become accelerated in the inflow convection-electric field $E_{y}$ along the shock front. The magnetic field of the current carried by the accelerated ions causes the magnetic foot in front of the shock ramp. The shock electrons are accelerated antiparallel to $E_{x}$ perpendicular to the magnetic field. The shock electrons perform an electric field drift in the $y$-direction in the crossed $E_{x}$ and compressed $B_{z 2}$ fields which leads to an electron current $j_{y}$ along the shock. These currents are sources of free energy which drives various instabilities in different regions of the perpendicular shock 
which retards it before it reaches the ramp. It is also clear that the foot ion current, which is essentially a drift current in which only the reflected newly energized ion component participates, constitutes a source of free energy. ${ }^{16}$ In its proper frame, it violates the energetic minimum state of the inflowing plasma. Being a source of free energy, the ion-drift current serves as a source for excitation of waves. Via these waves it contributes to filling the gap of the lacking dissipation in the foot region. However, in a quasi-perpendicular shock there are other sources of free energy as well which are not restricted to the foot region.

Figure 10 shows a sketch of some of the different free-energy sources and processes across a quasi-perpendicular shock. In addition to the shock-foot current and the presence of the foot-ion beam, the shock ramp is of finite thickness. It contains a charge separation electric field $E_{x}$ which in the supercritical shock is strong enough to reflect the lower energy ions. In addition it accelerates electrons downstream, thereby, in addition, deforming the electron distribution function.

The presence of this field, which has a substantial component perpendicular to the magnetic field, implies that the magnetized electrons, with their gyro radii $r_{c e}<\Delta_{\mathrm{sh}}$ being shorter than the shock-ramp width, experience an electric drift $V_{y e}=-E_{x} / B_{z 2}$ along the shock in the ramp which causes a substantial electron drift current $j_{y e}=$ $-e N_{e, \text { ramp }} V_{y e}=e N_{e, \text { ramp }} E_{x} / B_{z 2}$ in the $y$-direction and, again, contributes to the magnetic field. At maximum current the field is roughly $B_{z} \sim \mu_{0} j_{y e} \Delta_{\text {sh. }}$. The electron current region may be narrower than the shock ramp width, of the order of the electron skin depth $c / \omega_{p e}$. However, as long as we do not know the number of magnetized electrons which are involved in this current nor the width of the electric field region (which must be less than an ion gyro-radius because of ambipolar effects) the above estimate is good enough. This magnetic field of the electron-drift current causes an overshoot in the magnetic field in the shock ramp. When this current becomes strong, it contributes to current-driven cross-field instabilities such as the MTSI or the lower-hybrid instability.

Finally, the mutual interactions of the different particle populations that are present in the shock at its ramp and behind provide other sources of free energy. A wealth of instabilities and waves is thus expected to be generated inside the shock. To these micro-instabilities add the longer wavelength instabilities which are caused by the plasma and field gradients in this region. These are usually believed to be less important, because the crossing time of the shock is shorter than their growth time. However, some of them may propagate along the shock and have therefore substantial time to grow and modify the shock profile. In the following we turn to the discussion of numerical investigations of some of these processes, reviewing their current state and provide a comparison with observations.

\footnotetext{
16 This is so because the accelerated foot-ions that drift tangentially along the shock ramp constitute an ion beam. This ion beam propagates in a direction perpendicular to the upstream flow. Such an ion beam implies that the plasma in the foot is not in thermal equilibrium. The excess energy stored in the beam must be dissipated by some instability mechanism in order to restore the thermal state of the upstream medium.
} 


\subsubsection{Shock potential drop}

One of the important shock parameters is the electric potential drop across the shock ramp-including a possible potential drop across the shock foot. This potential drop is not necessarily a constant but changes with location along the shock normal; we have already noted that it is due to the different dynamical responses of the inflowing ions and electrons over the scale of the foot and ramp regions. Its theoretical determination is difficult. However, when going to the de Hoffmann-Teller frame, the bulk motion of the particles is only along the magnetic field, in the stationary electron equation of motion the $\mathbf{V}_{e} \times \mathbf{B}$-term drops out and, to first approximation, the cross shock potential is given by the pressure gradient (neglecting any contributions from wave fields). The expression is then simply

$$
\Delta \Phi(x)=\int_{0}^{x}\left[e N_{e}(n)\right]^{-1}\left[\nabla \cdot \mathrm{P}_{e}(n)\right] \cdot \mathrm{d} \mathbf{n}
$$

Integration is over $n$ along the shock normal $\mathbf{n}$. Under gyrotropic conditions, valid at length scales longer than an electron gyroradius (and thus well satisfied in a shock), the electron pressure tensor is $\mathrm{P}_{e}=P_{e \perp} \mathrm{I}+\left(P_{e \|}-P_{e \perp}\right) \mathbf{B B} / B B$. Taking into account that $\mathbf{E} \cdot \mathbf{B}$ is invariant, the shock potential can be expressed in terms of the gradient in the electron magnetic moment $\mu_{e}=T_{e \perp} / B$ (Goodrich and Scudder 1984) as

$$
e \Delta \Phi(x) \simeq \Delta\left(T_{e \|}+T_{e \perp}\right)-\int_{0}^{x} \mathrm{~d} n B(n)\left[\mathrm{d} \mu_{e}(n) / \mathrm{d} n\right]
$$

When the electron magnetic moment is conserved (which is the case only in the absence of interaction of the electrons with waves), the last term disappears, yielding a simple relation for the potential drop $e \Delta \Phi(x) \simeq \Delta\left(T_{e \|}+T_{e \perp}\right)$ as the sum of the changes in electron temperature. The perpendicular temperature change can be expressed in terms of the compression ratio of the magnetic field as $\Delta T_{e \perp}=T_{e \perp, 1} \Delta B / B_{1}$. The parallel change in temperature provides more problems. One could express it in terms of the temperature anisotropy $A_{e}=T_{e \|} / T_{e \perp}$, and afterwards vary $A_{e}$. But this depends on the particular model. It is more important to note that this adiabatic estimate of the potential drop does not account for any dynamical processes which generate waves and substructures in the shock. Thus, it just gives a hint on the order of magnitude of the potential drop across the foot-ramp region in quasi-perpendicular shocks.

\subsubsection{Observational evidence: Earth's bow shock wave}

As an example of measurements in situ of a supercritical quasi-perpendicular shock, Fig. 11 shows observations from a satellite crossings of Earth's bow shock wave (magnetosonic Mach number $\mathscr{M}_{m s} \sim 4$.2) by the two spacecraft ISEE 1 (upper block of the figure) and ISEE2 (lower block of the figure) from upstream to downstream in a short sequence only minutes apart. In spite of some differences occurring on the 

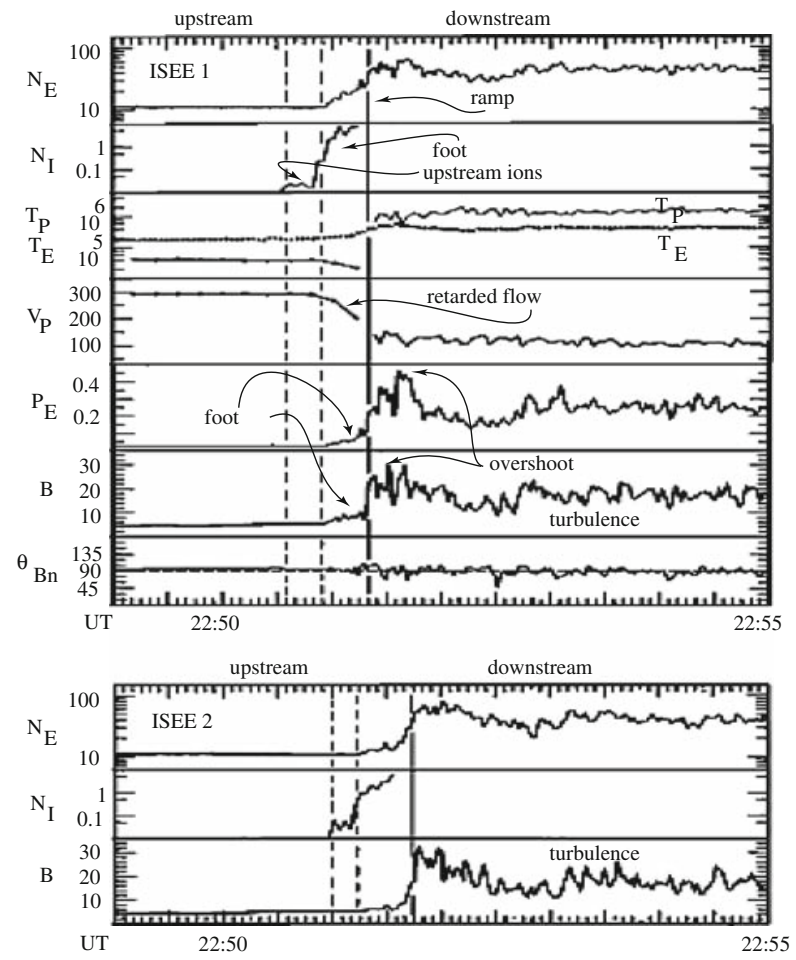

Fig. 11 Time profiles of plasma and magnetic field parameters across a real quasi-perpendicular shock, Earth's bow shock that had been crossed by the ISEE 1 and 2 spacecraft on November 7, 1977 (after Sckopke et al. 1983, courtesy American Geophysical Union). The measurement are typical for a quasi-perpendicular shock. $N_{E}$ is the electron density, $N_{I}$ the reflected ion density, both in $\mathrm{cm}^{-3}, T_{p}, T_{E}$ are proton and electron in K. $V_{P}$ is the proton (plasma) bulk velocity in $\mathrm{kms}-1, P_{E}$ electron pressure in $10^{-9} \mathrm{Nm}^{-2}, B$ the magnitude of the magnetic field in $\mathrm{nT}$, and $\theta_{B n}$. The vertical lines mark the first appearance of reflected ion, the outer edge of the foot in the magnetic profile, and the ramp in the field magnitude, respectively. The abscissa is the Universal Time UT referring to the measurements. The upper block are observations from ISEE 1, the lower block observations from ISEE 2

short time scale, the two shock crossings are about identical, identifying the main shock transition as a spatial and not as a temporal structure. Temporal variations are nevertheless visible on the scale of a fraction of a minute.

From top to bottom the figure shows the electron density $\left(N_{E}\right)$, energetic ion density $\left(N_{I}\right)$, proton and electron temperatures $\left(T_{P}, T_{E}\right)$, bulk flow velocity $\left(V_{P}\right)$, electron pressure $\left(P_{E}\right)$, magnetic field $(B)$, and $\theta_{B n}$. The latter is close to $90^{\circ}$ prior to shock crossing (in the average $\theta_{B n} \sim 85^{\circ}$ ), and fluctuates afterwards around $90^{\circ}$, identifying the shock as quasi-perpendicular. Accordingly, the shock develops a foot in front of the shock ramp as can be seen from the slightly enhanced magnetic field after 22:51 UT in ISEE 1, similarly in ISEE 2, and most interestingly also in the electron pressure. At the same time the bulk flow velocity starts decreasing already, as the result of interaction and retardation in the shock foot region. The foot is also visible in the electron density which increases throughout the foot region, indicating the presence 
of electrons which, as is suggested by the increase in pressure, must have been heated or accelerated.

The best indication of the presence of the foot is, however, the observation of energetic ions (second panel from top). These ions are first observed some distance away from the shock but increase drastically in intensity when entering the foot. These are the shock-reflected ions which have been accelerated in the convection electric field in front of the shock ramp. Their occurrence before entrance into the foot is understood when we realize that the shock is not perfectly perpendicular. Rather, it is quasi-perpendicular such that part of the reflected ions having sufficiently large parallel upstream velocities can escape along the magnetic field to a distance larger than the average upstream extension of the foot. For nearly perpendicular shocks, this percentage is small.

The shock ramp in Fig. 11 is a steep wall in $B$ and $P_{E}$, respectively. The electron temperature $T_{E}$ increases only moderately across the shock while the ion temperature $T_{P}$ jumps up by more than one magnitude, exceeding $T_{E}$ downstream behind the shock. This behaviour is due to the accelerated returning foot-ions which pass the shock. $P_{E}, B$, and $N_{E}$ exhibit overshoots behind the shock ramp proper. Farther away from the shock they merge into the highly fluctuating state of lesser density, pressure, and magnetic field that can be described as some kind of turbulence. Clearly, this region is strongly affected by the presence of the shock which forms one of its boundaries, the other boundary being the obstacle which is mainly responsible for the formation of the shock.

\subsubsection{Quasi-perpendicular shock scales}

For a quasi-perpendicular shock propagating and evolving in a high- $\beta$ plasma $^{17}$ there is a hierarchy of such scales available. These scales can be organized with respect to the different regions of the shock.

1. The macroscopic scale of the foot region, which determines the width of the foot, is the ion gyroradius based on the inflow velocity $r_{c i, 1}=V_{1} / \omega_{c i, 1}$. With the slight modification of replacing the upstream magnetic field with the (inhomogeneous) ramp magnetic field $B_{r}(x)$ this also becomes approximately the scale of the macroscopic electric potential drop in the ramp, $\Delta_{\phi, r} \sim r_{c i, r} \sim V_{1} / \omega_{c i, r}$.

Other scales are

2. the ion inertial length $c / \omega_{p i}$, which is also a function of space inside the ramp because of the steep density increase $N(x)$. This determines the dispersive prop-

$17 \beta=2 \mu_{0} n T / B^{2}$ refers to the normalised thermal energy of the flow, $\beta_{\text {kin } \perp}=2 \mu_{0} N m_{i} V_{n}^{2} / 2 B^{2} \equiv$ $\mathscr{M}^{2}>1$ implies that the kinetic energy in the flow exceeds the magnetic energy; the flow dominates the magnetic field, which is transported by the flow. In plasmas with $\beta_{\text {kin } \perp}=\mathscr{M}^{2}<1$, the magnetic field dominates the dynamics, and shock waves perpendicular to the magnetic field cannot evolve. Parallel shocks are basically electrostatic in the $\beta_{\text {kin } \perp} \ll 1$-case. They can evolve when the flow is sufficiently fast along the field, as is observed in the auroral magnetospheres of the magnetized planets in the heliosphere. On the other hand, at large Mach numbers and $\beta \gtrsim 1$ conditions, shocks do exist, as the examples of the solar wind and similar high speed stellar winds show. 
erties of the fast magnetosonic wave which is locally responsible for steeping and shock ramp formation;

3. the thermal ion gyrodradius $r_{c i}=v_{i} / \omega_{c i}$. This determines the transition from unmagnetized to magnetized ions and from nonadiabatic to adiabatic heating of the ions;

4. the density gradient scale $L_{P}=\left(\nabla_{x} \ln P\right)^{-1}$. This determines the importance of drift waves along the shock which, when excited, structure the shock in the third dimension perpendicular to the shock normal and the magnetic field;

5. the electron inertial length $c / \omega_{p e}$. This is the scale length of whistlers which are excited in front of the shock and are generally believed to play an essential role in shock dynamics;

6. the thermal electron gyroradius $r_{c e}=v_{e} / \omega_{c e}$. This determines whether electrons behave magnetized or nonmagnetized. In the shock they are usually magnetized under all conditions of interest. However, when nonadiabatic heating becomes important for electrons it takes place on scales comparable to $r_{c e}$;

7. the Debye length $\lambda_{D}$. This determines the dispersive properties of ion acoustic waves which are responsible for anomalous resistivity and for smaller scale density substructures in the shock, such as the phase space holes mentioned earlier which evolve on scales of several Debye lengths. It also determines the scales of the Buneman two-stream (BTS) and modified two-stream (MTS) instabilities which are the two most important instabilities in the shock foot.

The importance of these scales has been discussed by (Kennel et al. 1985) assuming that some anomalous resistance $\eta$ has been generated in the plasma. The dispersive fast magnetosonic wave velocity is $c_{m s}^{2}=c_{\mathrm{ia}}^{2}+V_{A}^{2} /\left(1+k^{2} R^{2}\right)$ with $R=R_{\eta}=$ $\left(\eta / \mu_{0}\right)(k / \omega)$ for $\eta \neq 0$, and $R=\lambda_{e}=c / \omega_{p e}$ for $\eta \rightarrow 0$. Here, the macroscopic scale of shock formation enters through $R$ which, in the collisionless case, becomes the electron skin depth $\lambda_{e}$. Starting from infinitely far away from the shock, one seeks for spatially growing solutions of the linear magnetic disturbance $b_{z} \sim \exp (\lambda x)$ in the stationary-point equation

$$
R_{e}^{2} b_{z}^{\prime \prime}+R_{\eta} b_{z}^{\prime}=D b_{z}, \quad D \equiv\left(1-\mathscr{M}^{-2}\right) /\left(1-c_{\mathrm{ia}}^{2} / V^{2}\right)
$$

where $^{\prime} \equiv \partial / \partial x$. With $b_{z} \rightarrow 0$ for $x \rightarrow-\infty$, this yields for the spatial growth rate $\lambda>\approx \sqrt{D} / \lambda_{e}$, for $R_{\eta} \ll \lambda_{e}$. This expression identifies the approximate shock transition scale as being proportional to the electron skin depth, $\Delta_{\mathrm{sh}} \simeq c / \omega_{p e} D^{\frac{1}{2}}$, just what one intuitively would believe to happen for freely moving electrons and ions. Since the upstream sound speed $c_{\text {ia }} \ll V$ is small compared with the fast flow $V$, we have $D \approx$ $1-\mathscr{M}^{-2}$, and the shock ramp width in terms of $\lambda_{e}=c / \omega_{p e}$ becomes slightly larger

$$
\Delta_{\mathrm{sh}} \simeq \mathscr{M}\left(\mathscr{M}^{2}-1\right)^{-\frac{1}{2}} \lambda_{e}
$$

For large Mach numbers, the shock ramp width approaches $\lambda_{e}$, yielding a very short shock transition and steep ramp. However, we have already seen that, at large Mach numbers, the competition between dispersion and dissipation does not hold anymore in this simple way. 
With increasing wave number $k$, the fast magnetosonic mode merges into the whistler branch with its convex dispersion curve. This implies that dispersive whistler waves will outrun the shock, becoming precursors of the shock, a problem we have discussed in Sect. 2. Whistlers propagate only outside their resonance cone. The limiting angle between $\mathbf{k}$ and the magnetic field $\mathbf{B}$ for which the whistler outruns the shock is given by

$$
\theta_{\mathrm{wh}, \lim } \lesssim \cos ^{-1}\left[\mathscr{M}_{A}\left(m_{e} / m_{i}\right)^{\frac{1}{2}}\right]
$$

artificially limiting the Alfvénic Mach number for whistler mediated shocks to

$$
\mathscr{M}_{A}=V / V_{A}<43
$$

In one-dimensional simulations, with all quantities changing only along the shock normal $\mathbf{n}$, and the $\mathbf{k}$-vectors of all waves pointing along $\mathbf{n}$, one chooses angles between $(\mathbf{k}, \mathbf{n})$ and $\mathbf{B}$ larger than $\theta_{\text {wh, lim }}$ in order not to be obscured by whistlers. However, the maximum phase speed of whistlers does not exceed the Alfvén speed by much. Hence, a standing whistler precursor will be attached to the shock, as long as the upstream velocity is less than the maximum whistler speed. When the upstream velocity exceeds this velocity, phase standing whistlers become impossible. This happens at the critical whistler-Mach number that has been given earlier. The shock structure becomes more complicated then by the formation of shock substructures (Galeev et al. 1988) on scales $c / \omega_{c e}$, and the shock becomes non-stationary.

\subsubsection{The shock transition scale}

Determination of the shock foot scale is relatively easy both from observation and numerical simulations. From observations, it was determined first by Sckopke et al. (1983), who found that the foot scale is slightly less but close $\left(\sim 0.7 r_{c i \text {, refl }}\right)$ to the reflected ion gyroradius in quasi-perpendicular shocks. The reasons for this number have been elucidated by Schwartz et al. (1983) and are related to the reflected ions that couple to the upstream convection electric field when becoming accelerated. This can also be checked in simulations. Of more interest is the determination of the width of the shock transition, i.e., the width of the shock ramp which from theory is not well determined since it depends on several factors which can hardly be taken into account at once.

Knowledge of the shock-transition width is particularly important in its relation to the width of the electrostatic potential drop across the shock. There are essentially three transition scales, the magnetic scale $\Delta_{B}$, the density scale $\Delta_{N}$, and the electric potential scale $\Delta_{E}$. Since the shock is not in pressure equilibrium, the first two scales need not necessarily be proportional to each other. However, the electric field and density gradient scales might be related, so one expects that $\Delta_{N} \sim \Delta_{E}$ even though this might not necessarily be so, in particular not when instabilities arise which cause small-scale electric field gradients. One can, in principle, distinguish three different cases (Lembège et al. 1999), each of which describes different physics: 
1. $\Delta_{E} \gg \Delta_{B}$. This case has been reported to have been observed in Bow Shock crossings by Scudder et al. (1986) and Scudder (1995). The magnetic ramp is much steeper in this case than the observed structure of the electric field. The latter is smeared out over the foot and ramp regions. In this case the electrons will behave completely adiabatically, while the ions may be only partially magnetized or even non-magnetized.

2. $\Delta_{E} \sim \Delta_{B}$. This case deviates significantly from adiabatic behaviour of the electrons in the shock transition. Electron heating and motion are non-adiabatic, and the electron distribution is significantly disturbed (cf., e.g. Balikhin et al. 1995). Observations of such cases have been reported by Formisano and Torbert (1982).

3. $\Delta_{E} \ll \Delta_{B}$. This case, also called 'isomagnetic' transition (Eselevich 1982; Kennel et al. 1985), corresponds to shock transitions with electrostatic substructuring, known as subshocks.

The most recent experimental determination of the density transition scale was provided by Bale et al. (2003) using data from 98 Bow Shock crossings by the Cluster spacecraft quartet. Fitting a tanh-dependence to the average magnetic field and density profiles, these authors found that the shock ramp transition depended on Mach number when the transition was scaled in ion inertial units, while there was no dependence when scaled in ion gyroradii. This different scaling suggested that the correct scaling would be with gyroradius, since $(V / c)\left(\omega_{p i} / \omega_{c i}\right) \sim \mathscr{M}_{A}$.

This result has been put in question on the basis of full-particle PIC simulations ${ }^{18}$ that have been performed in one dimension but with correct ion-to-electron mass ratio $m_{i} / m_{e}=1,838$. The simulation used the Alfvénic Mach number range $3.2 \leq \mathscr{M}_{A} \leq$ 14 and a shock normal angle $\theta_{B n}=87^{\circ}$ in order to have a component of $k_{\|}$parallel to $\mathbf{B}$, but with small ratio $\omega_{p e} / \omega_{c e}=4$ in order to compromise computing requirements (Scholer and Burgess 2006). They are reproduced in Fig. 12 and show that a tanh $x$-fit neglects the entire shock ramp and takes account only the foot region. Correcting the above described measurements, it is thus found that the ramp thickness is just of the order of $\sim 1 \lambda_{i}=c / \omega_{p i}$ or less and decreases slightly with increasing Mach number. However, from the form of the density profile it seems clear that the shock ramp is basically determined by the overshoot, and one must take the overshoot magnetic field value in calculating the gyroradius. The convected gyroradius based on the overshoot magnetic field $B_{\mathrm{ov}}$ and measured in $\lambda_{i}$ is about constant, very close to unity. Thus the shock ramp scale is given by the convective ion gyroradius based on the overshoot magnetic field. One should, however, note that the computing power in the simulations

\footnotetext{
18 PIC is the acronym for Particle-In-Cell that is used in numerical simulations of many-body systems. Here such systems are shocked many-particle plasmas. In PIC plasma-simulations the orbits of a finite number $j=1,2,3 \ldots, \ell$ of macro-particles per simulation-cell are numerically followed by solving Newton's law $d \mathbf{v}_{j} / d t=\mathbf{F}_{j} / m_{j}$ for each of them separately, when the particles interact via the local Lorentz force $\mathbf{F}_{j}$ with their self-consistently generated electromagnetic fields. During the evolution of the orbits the particles jump from one cell to the next. Usually, being macro-particles, each of them represents a very large number of real particles which are assumed to have clumped together, have about similar dynamics, and perform about similar orbits for the time of the simulation. There are two types of PIC simulations, those where both electrons and ions are treated as particles, called full PIC simulations, and those where only the ions are treated as particles while the electrons are treated as a charge neutralising fluid. These latter simulations are called hybrid simulations.
} 

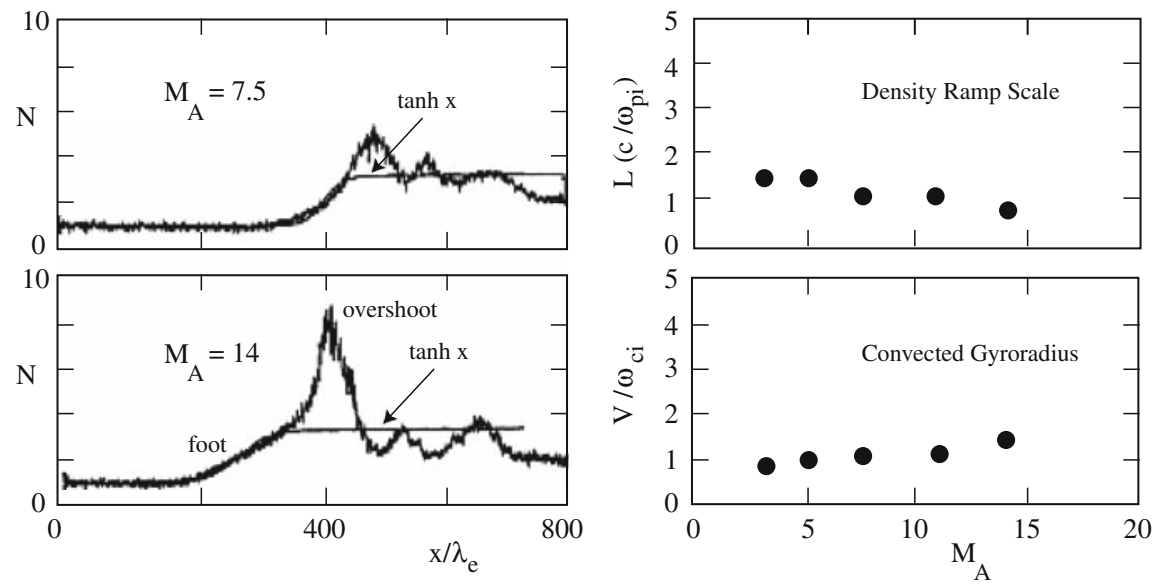

Fig. 12 Left: Density profile in two full PIC simulations of large Mach numbers. Indicated is the pronounced overshoot and the long extended foot. The straight lines are $\tanh x$-fits to the simulations showing the neglect of the overshoot and ramp during such fits which only account for the foot region. From fitting the ramp width the curves on the right are obtained. Right: Density ramp scales and convected ion gyroradii (in units of upstream inertial length) obtained in one-dimensional full particle PIC simulations of quasiperpendicular shocks (after Scholer and Burgess 2006) as function of Alfvénic Mach number. Use has been made of the full particle mass ratio $1,838, \theta_{B n}=87^{\circ}$, and $\omega_{p e} / \omega_{c e}=4$. The magnetic field used is that of the overshoot. One observes that the ratio of ion gyroradius to ion inertial length is constant. Also the scale of the ramp is about $\sim 1 c / \omega_{p i}$, supporting a narrow ramp. The simulations also show that the scale of the ramp sharpens with increasing Mach number

does not yet allow for larger ratios $\omega_{p e} / \omega_{c e}$ which may affect the result. Moreover, higher dimensional simulations would be required to confirm the general validity of those calculation and conclusions.

Hence, combining the observations of Bale et al. (2003) and the results of the simulation studies of Scholer and Burgess (2006) we may conclude that

- the scale of the shock foot is given by the upstream-convected ion gyroradius $r_{c i}=V / \omega_{c i, 1}$, based on the upstream field $B_{1}$,

- while the scale of the shock ramp is given by the ramp-convected ion gyroradius $r_{c i, \mathrm{ov}}=V / \omega_{c i, \mathrm{ov}}$, based on the value of the magnetic field overshoot $B_{\mathrm{ov}}$.

This is an important difference which can be taken as a golden rule for estimates of the structure of quasi-perpendicular shocks even though, of course, these values are dynamical values which change from position to position across the foot and ramp, and tangentially along the shock front. The scale differences are the reasons for the large upstream extension of the foot and the relative steepness of the shock ramp.

The observed constancy of the overshoot-magnetic field-based convective ion gyroradius $r_{c i} \propto V / B_{\text {ov }}$ with Mach number $\mathscr{M}_{A} \propto V$ can be understood when considering the about linear increase of the overshoot magnetic field $B_{\text {ov }} \propto \mathscr{M}$ with Mach number (or with upstream velocity $V$ ) which holds for supercritical Mach numbers $\mathscr{M}>\mathscr{M}_{\text {crit }}$ as long as $\mathscr{M}$ is not too large. At very large_-but still non-relativistic_-Mach numbers $\mathscr{M}<\mathscr{M}_{\max }$ the increasing steepness of the shock ramp and the increasing extension 
of the foot ultimately lead to the excitation of smaller scale structures in the ramp and the foot, which smear out any further increase in the overshoot.

The generation of these structures by a variety of instabilities might even turn the shock foot and ramp regions into regions where large anomalous collisions, and thus resistances, are generated as the result of wave-particle interactions. In this case, the shock returns to again becoming resistive. It prevents large numbers of reflected ions from passing across the steep shock ramp and the large shock potential. The kinetic energy of the reflected particle population is used for the generation of a broad wave spectrum which acts to scatter the particles around in the foot and ramp regions and, possibly, also up to some distance in the transition region behind the ramp. The particles do not transit across the shock for quite a long time. This kind of 'confinement of reflected particles' over long times can be sufficiently long to provide the heating and dissipation which is required for sustaining a resistive shock which, then, is the result of the combined action of ion viscosity and anomalous resistivity, i.e., anomalous collisions. In addition, the scattering of the trapped reflected particle population necessarily results in broadening of the distribution function, which is equivalent to plasma heating. In these interactions, some particles may become accelerated to high velocities. It then becomes possible that these particles provide the seed population for energetic particles which can be accelerated to high energies in the well-known shock-Fermi-one and shock-Fermi-two acceleration mechanisms.

So far the range of Mach numbers $\mathscr{M}_{\text {max }}<\mathscr{M}<\mathscr{M}_{\text {rel }}$ where this will happen is unknown, as it is hardly accessible to numerical simulations. However, the available simulations seem to point in this direction as long as the Mach numbers remain nonrelativistic. In relativistic shocks with $\mathscr{M}=\mathscr{M}_{\text {rel }}$, different effects arise which are not subject to our discussion at this time.

\subsection{Quasi-perpendicular shock reformation}

Supercritical shocks are not stationary. They do under certain conditions reform themselves quasi-periodically. In this process the shock is not destroyed but, on the contrary, it is kept intact in a periodically changing way.

\subsubsection{Mass-ratio dependence}

Figure 13, on its left, shows an example of the evolution of the magnetic field signature during the process of quasi-perpendicular shock reformation. The data have been obtained with a low ion-to-electron mass-ratio $\left(m_{i} / m_{e}<100\right)$ two-dimensional full-particle PIC simulation. On the right, the spatial structure of the magnetic shockramp field along the shock is shown at two different late reformation times. The left figure suggests that there is a distinct reformation cycle in this simulation. The right figure shows that, in addition, a distinct structure of the ramp/shock front evolves in a tangential direction which is far from being smooth. The shock not only reforms cyclically in time, it also develops ripples along its surface which travel like waves along the shock and modulate the shock amplitude. 

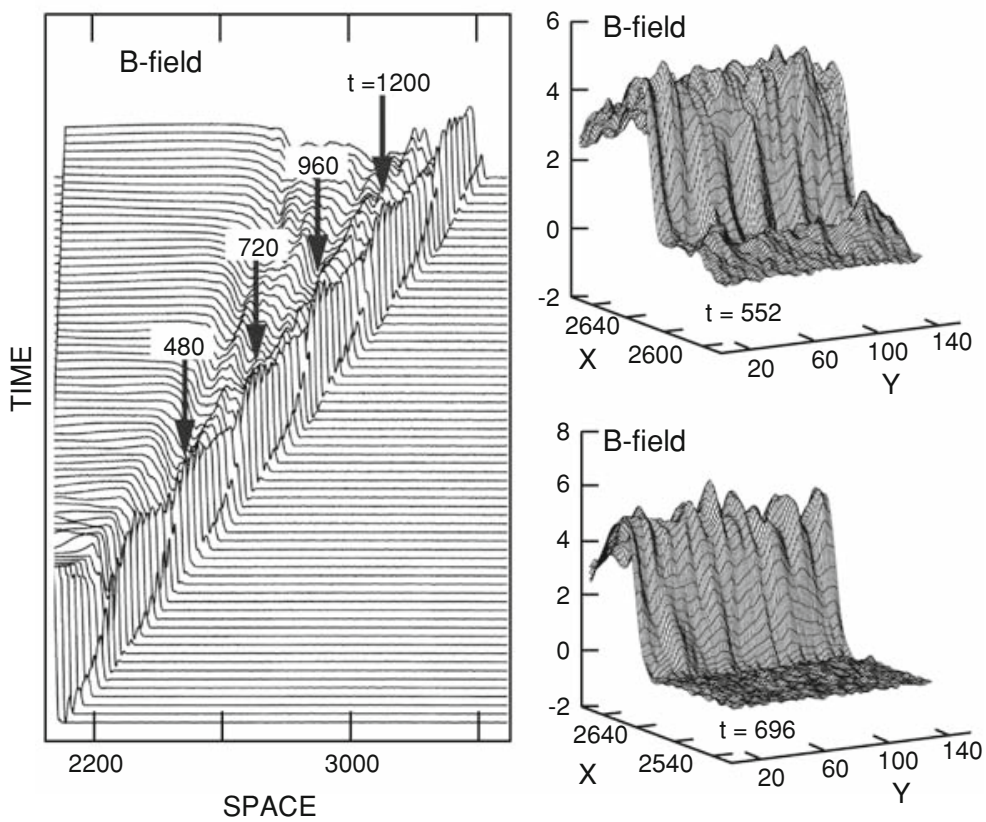

Fig. 13 Magnetic field from full particle PIC simulations of shock reformation (after Lembège and Savoini 2002, courtesy American Geophysical Union). Left: Reformation cycles of the magnetic field in the shock. Time is measured in inverse electron plasma frequencies $\omega_{p e}^{-1}$. The reformation times are indicated by the arrows in the plot with time given when the cycle is complete. Right: Tow snaphots in time of the view of the shock front in the magnetic field at reformation. The interesting finding is that the front in this two-dimensional view is not a smooth plane but is quite distinctly structured in space

Full particle electromagnetic PIC simulations with realistically large mass ratios have been performed only very recently (Matsukiyo and Scholer 2003, 2006a; Scholer et al. 2003; Scholer and Matsukiyo 2004) and only in one spatial dimension, showing that reformation occurs at small ion- $\beta_{i} \sim 0.2$. The shock is produced by injecting a uniform plasma from $-x$ and letting it reflect from a stationary wall at the right end of the simulation box. The plasma carries a uniform magnetic field in the $(x, z)$ plane, and plasma is continuously injected in the $+x$-direction. Since the right-hand reflecting boundary is stationary, the shock, which is generated via the ion-ion beam instability in the interaction of the incoming and reflected ion beams, moves to the left at a velocity that is given by the supercritical shock Mach number $\mathscr{M}_{A} \sim 4.5$. The upstream plasma has $\beta_{i}=\beta_{e}=0.05$, and the shock normal angle is $\theta_{B n}=87^{\circ}$.

Two runs of these simulations are shown in Fig. 14; one is for a mass ratio of 400, the other for a realistic mass ratio of 1,840. The left-hand side of the figure shows the time evolution of the nearly perpendicular magnetic field $B_{z}$, with time running in equidistant units upward on the ordinate and measured in ion cyclotron periods $\omega_{c i}^{-1}$, while space on the abscissa is in units of the electron inertial length $c / \omega_{p e}$. The shock is seen to move from right to left in this pseudo-three-dimensional representation. The magnetic profiles are strikingly similar for both mass ratios. In both cases a flat foot 

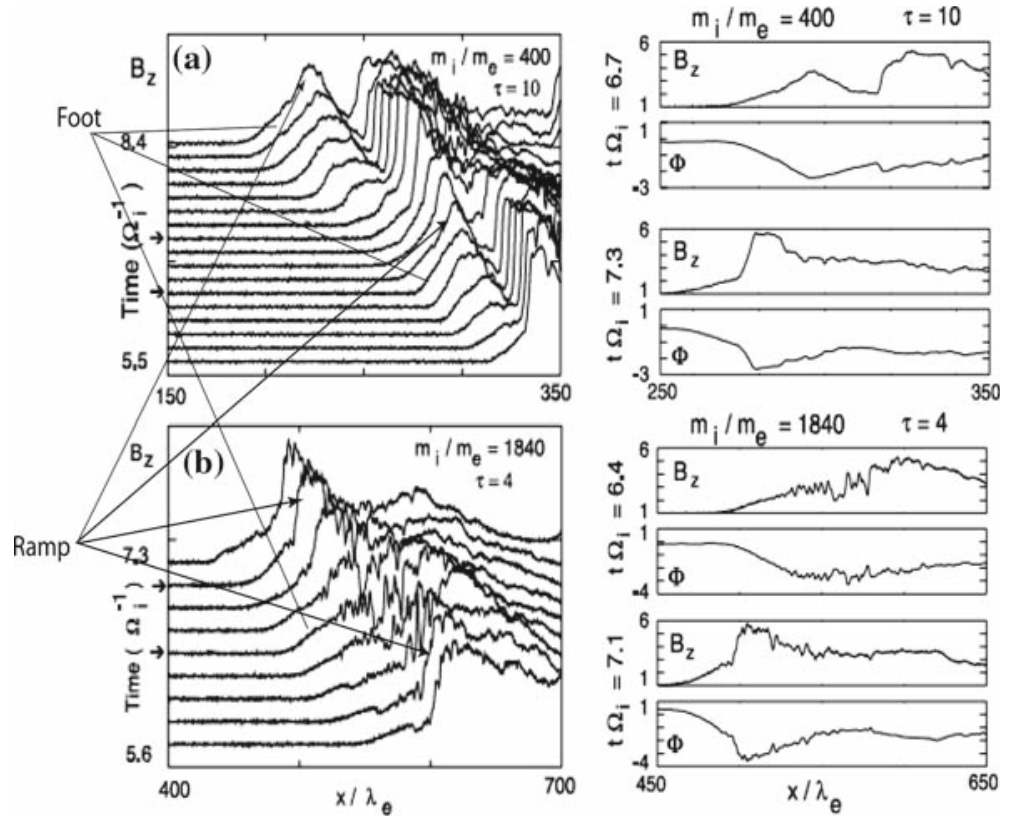

Fig. 14 Left: 1D-PIC simulations (after Scholer et al. 2003, courtesy American Geophysical Union) of quasi-perpendicular $\theta_{B n}=87^{\circ}$ shock reformation (mass ratios $m_{i} / m_{e}=400$ and 1,840). Time is in $\omega_{c i}^{-1}$, space in $\lambda_{e}$ and small $\tau=\omega_{p e^{2}} / \omega_{c e}^{2}$. Higher mass ratio show strong time evolution. Reformation is due to evolution of shock feet. Right: Spatial profiles at two time sections (see arrows on the left). The higher mass ratio shows structure in $B_{z}$ and shock potential $\Phi$. Potential drops appear in foot and ramp

develops in front of the steep shock ramp, caused by the shock-reflected ions. The magnetic field of the foot itself increases with time, with growth being strongest close to the upstream edge of the foot, until the foot field becomes so strong that it replaces the former shock ramp and itself becomes the new and displaced shock ramp. This is seen most clearly in the low mass-ratio panel. The foot takes over, steepens and replaces the shock. In addition the foot reflects ions and develops its own flat pre-foot region. This pre-foot readily evolves to become the next foot, while the old shock ramp merges into the downstream turbulence.

During reformation the shock progresses upstream. This progression is not a continuous motion at constant speed. Both the foot and the ramp jump forward in steps. One such step occurs at $t \omega_{c i}=7.6$ in the upper part on the left. Sitting in the shock frame one would experience some forward acceleration at this time, seeing the ramp running downstream like a magnetic wave the source of which apparently being the instantaneous shock ramp, while it is just the edge of the old shock foot. Hence the shock ramp and shock overshoot act as sources of a pulsating magnetic wave that is injected downstream from the shock with periodicity of roughly $\Delta t \sim 1.8 \omega_{c i}^{-1}$ for $m_{i} / m_{e}=400$, adding to the downstream turbulence.

Reformation also occurs, with some distinctions, for the realistic mass-ratio (lower left part). The magnetic profile is much more strongly disturbed, exhibiting stronger structuring. The foot region is considerably more extended in the upstream direction. 
The ramp is much steeper, and reformation is faster, happening on a time scale of $\Delta t \sim 1.3 \omega_{c i}^{-1}$, roughly $30 \%$ faster than in the low mass-ratio case. Reformation is, however, more irregular at realistic mass ratio with the property of reforming the shock ramp out of a long extended and relatively smooth shock foot which exhibits pronounced oscillations.

The right-hand side of the figure shows snapshots of two shock profiles at constant times for the two different mass-ratio simulations. The profile at $t \omega_{c i}=6.7$ has been taken when a well developed foot and ramp had been formed on the shock, the profile at $t \omega_{c i}=7.3$ is at the start of the new foot towards the end of the simulations. For low mass ratio, the foot profile is smooth, showing that the foot is produced by the accumulation of reflected ions near the upstream edge of the foot where the ions have the largest velocity in direction $y$ along the shock. This is where, during their upstream gyration in the upstream magnetic field, they orbit about parallel to the upstream convection electric field and gain the most energy. Here, the current density is highest due to the accumulation of reflected ions, retardation of ions from the inflow at this place, and due to the speeding-up of the reflected and retarded ions in the $y$-direction by the convection electric field $E_{y}$. All this maximises the current $j_{y}$ and the magnetic field $B_{z}$ close to the upstream edge of the foot. The electric potential exhibits its strongest drop in the foot region with a second smaller drop in the ramp. It is the electric field belonging to this potential drop that retards the plasma even before it arrives at the shock ramp. In contrast, for the short time when the shock ramp is well developed, the main potential drop is at the ramp and extends into the downstream region.

For realistic mass ratio, the foot- and ramp-transitions are both highly structured at $t \omega_{c i}=6.4$, exhibiting fluctuations in magnetic field and electric potential, but the electric potential drop extends all along the foot reserving a small drop only in the ramp. When the ramp has been reformed at $t \omega_{c i}=7.1$, the foot region still maintains a substantial potential drop, but $50 \%$ of the total drop is now in the ramp with the downstream potential recovering. This implies that lower energy electrons will become trapped in the overshoot region, an effect which is much stronger for realistic mass-ratios than for small mass-ratios and thus closer to reality.

\subsubsection{Ion dynamics}

Figure 14 gives a clear idea of the behaviour of ions in the reformation process. The corresponding ion phase space plots are shown in Fig. 15 for the two mass ratios $m_{i} / m_{e}=400$ (top) and 1,840 (bottom) respectively. Both plots show only the enlarged shock foot transition region over the same scale of $100 \mathrm{c} / \omega_{p e}$ for the same $\beta_{e}=0.2$, which has been kept constant in both simulations, while the $\beta_{i}$ has been changed. Only the normal component of the ion velocity is shown for the nearly perpendicular supercritical shock. In both plots the magnetic field $B_{z}$ is drawn as a thin continuous line showing the magnetic shock profile over the spatial distance $\Delta x$.

The upper (low-mass-ratio) low- $\beta_{i}$ panel shows the cold dense ion inflow at velocity $v_{i x} \sim 5$ (in units of the upstream Alfvén velocity) being retarded already to nearly Mach number 1 when entering the foot. This retardation is due to its interaction with the intense but cold (narrow in velocity space) reflected ion beam which is seen as the narrow negative $v_{x i}$-velocity beam originating from the shock ramp. This reflected 


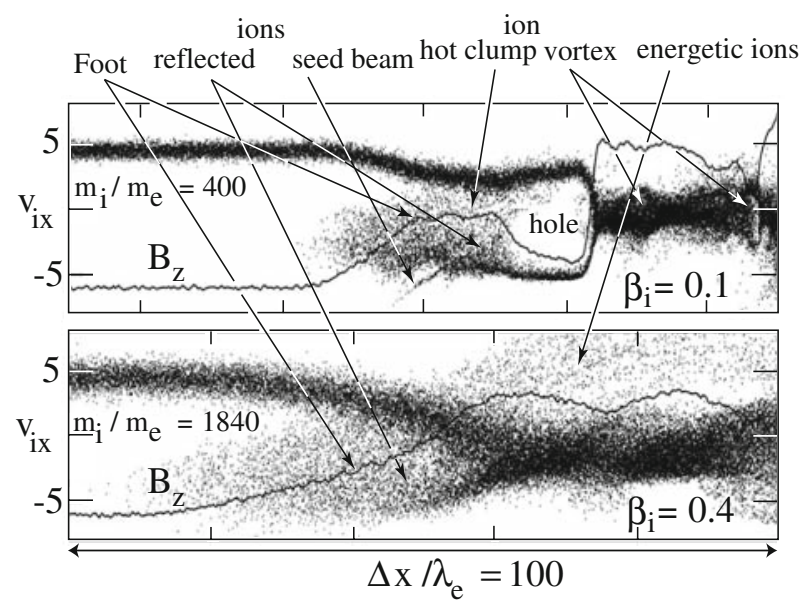

Fig. 15 Ion phase space for $\beta_{e}=0.2$ but different mass ratios and $\beta_{i}$ (after Scholer et al. 2003, courtesy American Geophysical Union) at same Mach number. Top: $\beta_{i}=0.1$ simulation. A low-field hot clumpvortex is formed which scatters reflected and upstream ions. Reformation cycles appear in the downstream distribution as ion vortices (holes). Bottom: Realistic mass ratio but $\beta_{i}=0.4$. Hot foot ions smear out the gap between inflowing and reflected ion beams. Large numbers of diffuse energetic ions appear in this case

ion beam needs a certain distance to interact with the upstream plasma inflow. This distance is the growth-length of the beam-beam excited waves. At this location the reflected ions are scattered into a hot ion clump in addition to being turned around by gyration. Both effects cause a reduction in velocity $v_{x}$ of the reflected ions which, being accelerated in the convection electric field, turn to flow in the $y$ direction, causing the magnetic bump that develops in this region of the foot. In the $\left(v_{x}, x\right)$-plane the reflected ions close with the upstream flow into a hot ion ring distribution (vortex) just in front of the ramp, of which the hot ion clump that brakes the inflow is the upstream boundary. Behind the ramp, which is the point of bifurcation of the ion distribution, i.e., the location where the reflection is at work, a broad hot ion distribution arises which at some locations shows rudimentary remains of ion vortices from former reformation cycles. Their magnetic signatures are the dips seen in the magnetic field. The next reformation cycle can be expected to completely close the ion vortex in the foot and to transform the ramp from its current position to the position of the foot. The first sign of this process is already seen in the foot ion distribution, which shows the birth of a faint new reflected ion beam at high negative speeds. This beam is not participating in the formation of the ring but serves as the seed of the newly reflected population. A similar behaviour is found in realistic mass-ratio simulations as long as $\beta_{i}$ is small, as is obvious from the realistic mass-ratio magnetic field shown in Fig. 14. As long as $\beta_{i}$ remains small, the shock also undergoes reformations for realistic mass ratios. In other words, as long as the plasma is relatively cool the real shocks found in nature should develop feet which at later times quasi-periodically become the shock ramp.

This changes completely, when $\beta_{i}$ increases, as is suggested by the lower panel in Fig. 15. There, a realistic mass ratio has been assumed, but $\beta_{i}=0.4$. No reformation is observed, at least not during the simulation time. Instead, the shock develops a very 
long foot region that extends upstream twice as far as for low- $\beta_{i}$. The high ion temperature smears out the reflected ion population over the entire gap region between upstream and reflected beam regions, and no vortex can develop. This implies that the foot remains smooth and does not evolve into a secondary ramp.

Reformation will thus be suppressed only when the thermal speed $v_{i}$ of the ions is large enough to bridge the gap between the reflected and incoming ion beams, i.e. large enough to fill the hole. Semi-empirically one can establish a condition for shock reformation as $v_{i}<\alpha V_{n 1}$ when taking into account that the normal speed of incoming ions is specularly turned negative. Since this is never exactly the case, the coefficient will roughly be in the interval $1.5<\alpha<2$. This condition for reformation to occur can be written as $\beta_{i}<\alpha \mathscr{M}_{A}^{2}$, where the Alfvénic Mach number is defined on $V_{1 n}$. The larger the Mach number the less suppression of reformation will play a role, and at high Mach numbers one expects that either reformation is a normal process or that other time-dependent processes set on which lead to a non-stationary chaotic and unpredictable state of shock reformation. As we have argued earlier this is quite normal as the shock is thermodynamically and thermally not in equilibrium: it is a region where electrons and ions have violently different temperatures; it is not in pressure equilibrium; upstream and downstream temperatures are different; and it hosts a number of non-Boltzmannian phase space distributions all concentrated in a small volume of real space. Under such conditions stationary states will occur only exceptionally. Recent hybrid simulations undertaken to determine the reflected ion density needed for reformation to occur completely neglect the electron mass effect and must therefore be taken with caution.

Shock reformation turns out to be an important fact in quasi-perpendicular shock physics. Its sensitivity to the mass ratio $m_{i} / m_{e}$ indicates its dependence on the dynamics of both populations, electrons and ions, i.e., it depends on the spectrum of waves that can be excited in the interaction between the inflowing and reflected plasma components. In the following we briefly discuss the relevant wave modes generated in this interaction before returning to their effect on shock formation, reformation and stability.

\subsubsection{Ion instabilities}

In quasi-perpendicular shocks the most important wave that is excited by the interaction between inflowing and reflected ions is the whistler mode. We already noted that below the critical whistler Mach number $\mathscr{M}_{\mathrm{wh}}=\frac{1}{2} \sqrt{m_{i} / m_{e}}\left|\cos \theta_{B n}\right|$ this is important only in producing waves upstream of the shock in the foot region. Since for realistic mass ratio $m_{i} / m_{e}=1,840$ and, for instance, $\theta_{B n}=87^{\circ}$ this is quite small, $\mathscr{M}_{\text {wh }} \simeq 1$.2. Otherwise no standing whistlers can be expected. Thus one is restricted to rather low Mach numbers for this effect, putting the problem into the subcritical box. However, when going to higher Mach numbers and taking realistic mass ratios, ion waves might still become excited. This has been checked in simulations (Scholer and Burgess 2006) who found the expected standing whistlers for $\mathscr{M}<\mathscr{M}_{\text {wh }}$, while for $\mathscr{M}>\mathscr{M}_{\text {wh }}$ ordinary shock reformation with some non-phase-standing whistlers have been seen, which near the ramp trap the ions and contribute to the ion-vortex formation discussed above. 
In order to see what kind of waves are excited during the whistler cycles, a separation of the magnetic wave spectrum $B_{y}$ into positive $B_{y}^{+}$and negative $B_{y}^{-}$helicity components has been performed for a $\mathscr{M}_{A}=9, \theta_{B n}=70^{\circ}$ simulation run. Figure 16 shows the result. The negative helicity waves $B_{y}^{-}$propagate toward the shock, i.e. to the right. After correcting for the convection velocity which is also to the right, these waves turn out to be left-hand polarised. The lower panel shows positive helicity $B_{Y}^{+}$waves propagating to the left, so they are upstream propagating waves and are also left-hand polarised. The positive helicity waves have longer wavelength than negative helicity waves. They propagate close to the shock speed upstream. They are thus almost standing in the shock frame. These are the upstream left-hand polarised (ion beam and not electron temperature anisotropy driven) whistlers. The downstream propagating negative helicity waves are no whistlers. They are caused in quite a different way which is related to the electromagnetic MTSI which we will discuss in relation to electron waves.

Stability of the ramp is a question that is not independent of the stability of the foot as both are closely connected by the reformation process of the quasi-perpendicular shock front. Waves that contribute to the stability of the shock ramp are related to the above mentioned waves that propagate downstream from the foot and reach the shock; on the other hand, the shock front may become unstable with respect to low frequency interface waves, which are excited in the shock ramp gradients and propagate along the shock surface. They may be responsible for the rippling of the shock front.

\subsubsection{Electron dynamics: two-stream effects}

Observations in situ of the quasi-perpendicular Earth's bow shock wave (plotted in Fig. 17) indicate that electrons are strongly heated when crossing the shock from upstream to downstream. Their reduced (field-parallel) distribution changes from Maxwellian to hot flat-top type with the intermediate distribution observed in the shock ramp showing signs of a shock-reflected electron beam. This heating and electron reflection is typical for supercritical shocks.

When talking about the dynamics of electrons, hybrid simulations cannot be used anymore. Instead, one must return to the more involved full particle PIC simulation codes (or to Vlasov codes), short time scales of the order of the electron gyro-period $\omega_{c e}^{-1}$ or electron plasma period $\omega_{p e}^{-1}$ must be resolved, and resolution of spatial scales of the order of the electron inertial $\lambda_{e}$ and Debye scales $\lambda_{D}$ is required. Reliable simulations of this kind became available only within the last decade with the improved computing capacities. Figure 18 shows two representative one-dimensional PIC simulations of the quasi-perpendicular magnetic shock profile and ion phase space with realistic mass ratio $m_{i} / m_{e}=1,840$ for medium Mach number $\mathscr{M}=4.5$. At an angle $\theta_{B n}=90^{\circ}$ (upper panels) we observe the formation of a foot with large ion holes, as described above. However, at a slightly inclined shock-normal angle $\theta_{B n}=87^{\circ}$ (lower panels) foot formation is completely different. There is no ion accumulation at the edge of the foot, and the foot is smooth and extended while being highly irregular in the magnetic field as well as in the ion behaviour in phase space. Drastic deceleration 


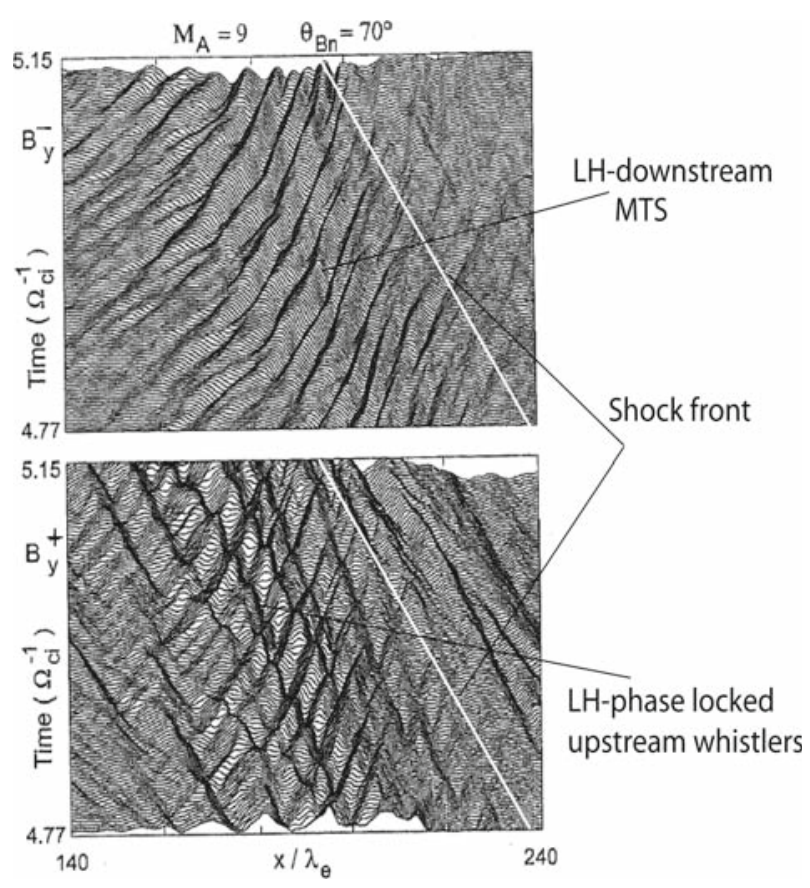

Fig. 16 1D-PIC simulations (realistic mass ratio, $\mathscr{M}_{A}=9, \theta_{B n}=70^{\circ}$ ) in non-reformation whistler regime (after Scholer and Burgess 2006). Top: Negative helicity waves $B_{y}^{-}$propagating to the right are left-hand polarised short wavelength waves moving downstream toward the shock being absorbed in shock transition. Bottom: Positive helicity waves $B_{y}^{+}$moving upstream (being left-hand circularly polarised), being upstream phase-locked whistler precursors of decaying amplitude and long wavelength. Some interference is seen on these waves. Their left-hand polarisation identifies them as ion-beam excited whistlers not due to electron temperature anisotropy

of the upstream ions is observed. The ions form many smaller scale holes, and the magnetic profile becomes broad with a long highly disturbed ion mixing zone. A most interesting observations relates to diffuse accelerated energetic ions, which in the perpendicular case are sparsely present. In the inclined case acceleration is quite strong, generating a broad diffuse energetic ion distribution all over the foot and shock transition region. This behaviour is due to instabilities in the shock foot and shock transition in which the electrons are deeply involved.

Papadopoulos (1988) proposed that in the foot region of a perpendicular highly supercritical shock the velocity differences between reflected ions and electrons from the upstream plasma inflow should be responsible for the excitation of the BTS instability, thus heating the electrons, generating anomalous conductivity and causing dissipation of flow energy which contributes to shock formation. Shimada and Hoshino (2000) and Schmitz et al. (2002) building on this idea performed full particle PIC simulations in strictly perpendicular shocks discovering that the BTS instability can indeed work in the foot region of the shock and can heat and accelerate the electrons. Shimada and Hoshino (2000) initiated their one-dimensional strictly perpendicular 


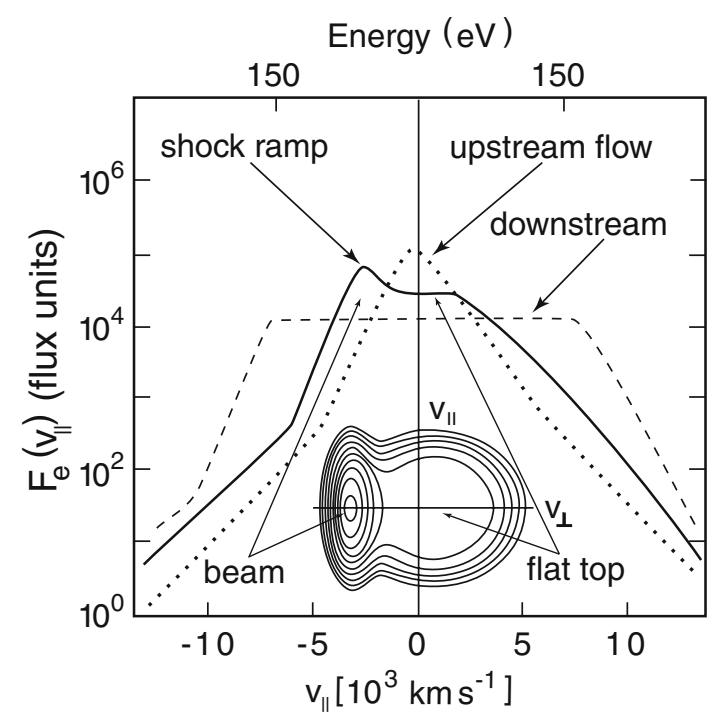

Fig. 17 Successive reduced parallel electron distributions $F_{e}\left(v_{\|}\right)$in the supercritical Earth's bow shock (ISEE 2 on December 13, 1977). The transition from the Maxwellian-plus-halo upstream flow distribution through the shock ramp distribution to the shock downstream distribution is monitored. The shock ramp distribution is intermediate in evolving from beam into a flat-top distribution. It contains in its upstream directed part a shock-reflected electron beam of velocity of a few $1,000 \mathrm{~km} \mathrm{~s}^{-1}$ which is sufficiently fast to excite electron plasma waves (after Gurnett 1985, courtesy American Geophysical Union)

$\left(\theta_{B n}=90^{\circ}\right)$ simulations for a small mass ratio of $m_{i} / m_{e}=20, \beta_{i}=\beta_{e}=0.15$, and Alfvénic Mach numbers $3.4 \leq \mathscr{M}_{A} \leq 10.5$.

Figure 19 shows their results on the right in expanded view. The electron phase space shows the development of electron holes which are generated by the Buneman two stream instability. The electrostatic field $E_{y}$ in the bottom panel shows the bipolar electric field structure caused by the holes with zero mean. In the hole, $E_{y}$ assumes large values. This is the behaviour expected for solitons and BGK modes. Such structures trap and heat electrons and accelerate passing electrons to high speeds. Both are seen in the simulations near the shock: three such holes are completely resolved, with amplitude decreasing closer to the shock ramp. They contain a small number of trapped electrons over a wide range of speeds which, on the gross scale in the left panel, fakes the high temperature of the electrons. In addition the electron velocity shows two accelerated populations, one with positive velocity about 2-3 times the initial electron speed, the other a reflected component with velocity almost as large as the positive component but in the opposite direction, suggesting that the electron current in the holes is almost compensated for by the electron distribution. Further heating of electrons in the ramp is caused by many overlapping holes as suggested by the structures in the inflowing and reflected ion distributions, which also strongly interact with the electric field of the holes. The incoming ion component in the first hole has a dip in the velocity due to retardation by the hole. Also the reflected ion component has strong distortions in its backward directed velocity when encountering a hole. Similar scattering of the incoming ion component characterises the 


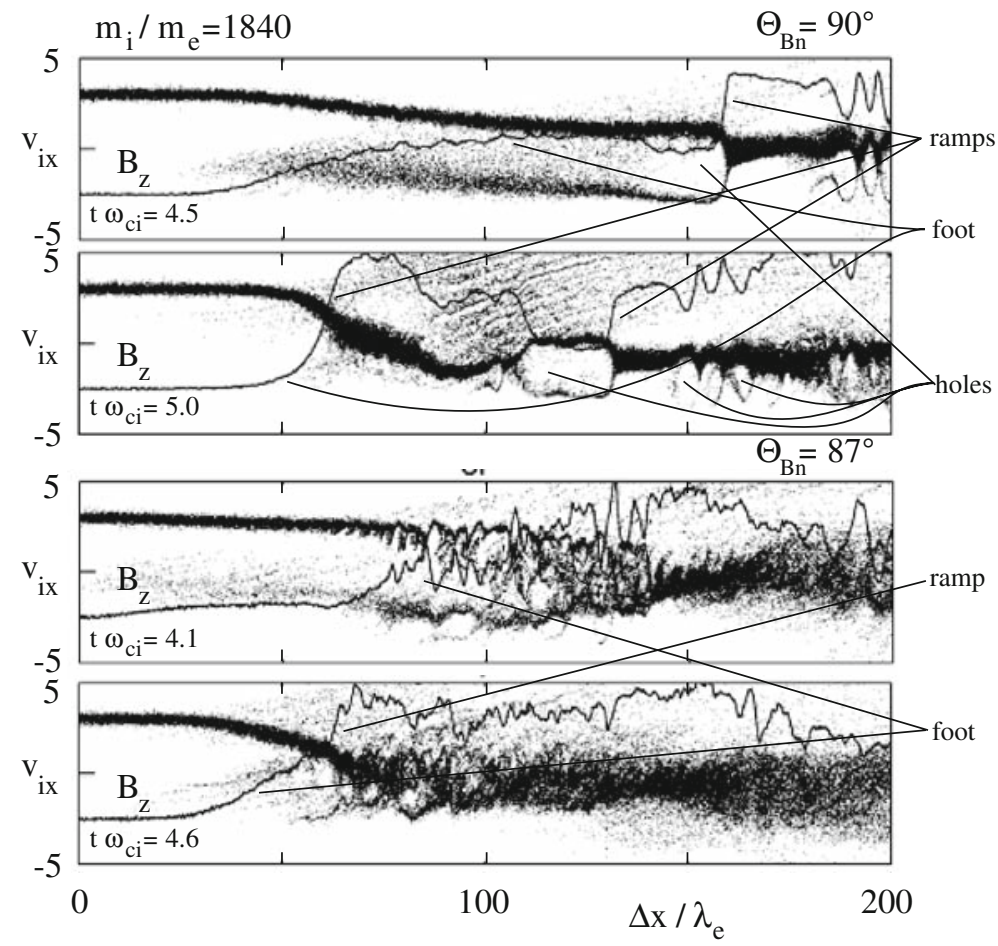

Fig. 18 The completely different reformation behaviour of shocks in one-dimensional PIC simulations with realistic mass ratio of 1,840 for strictly perpendicular and oblique quasi-perpendicular shocks at exactly the same parameter settings and scales. Shown is the magnetic field $B_{z}$ and the ion phase space at two subsequent simulation times for each of the respective simulations with $\mathscr{M}=4.5$. Since the evolution is different in both cases, the $x$-coordinate is given as a relative scale not in $x$ but for the same interval lengths in $\Delta x$ for the instance when reformation takes place in both cases (compiled from Matsukiyo and Scholer 2003, courtesy American Geophysical Union). Top: Reformation at $\theta_{B n}=90^{\circ}$ at two times showing the evolution of the foot in the magnetic field and the taking-over of the ramp by the foot while a new foot evolves. This process is governed by the BTS instability. Large holes evolve on the ion distribution. Note the correlation of the ion holes with depressions in the magnetic field, In the second panel the old ramp is still visible as the boundary of the large ion hole. Farther downstream many holes are seen, each of them corresponding to a magnetic depression, and the regions between characterised by magnetic overshoots. Bottom: The corresponding evolution at $\theta_{B n}=87^{\circ}$. High variability of the shock profile is observed which is identified as being due to the large amplitude MTS-waves travelling into the shock. The foot region is extended and very noisy both in the magnetic field and ion distributions, the latter being highly structured. The foot is extended much longer than in the perpendicular case. The two bottom panels might also show signatures of wave breaking in the ion velocities when groups of ions appear which overturn the main flow in forward downstream direction

ramp, suggesting the presence of a large number of electric field structures which are related to the highly fluctuating electric field component in the ramp in the bottom panel.

The magnetic signature confirms that the reflection of the main incoming ion beam takes place at the location of the magnetic overshoot and not in the shock ramp in the strictly perpendicular supercritical shock. The actual ramp region is narrow. Its width is only of the order of $\Delta \sim(1-2) c / \omega_{p e}$. 

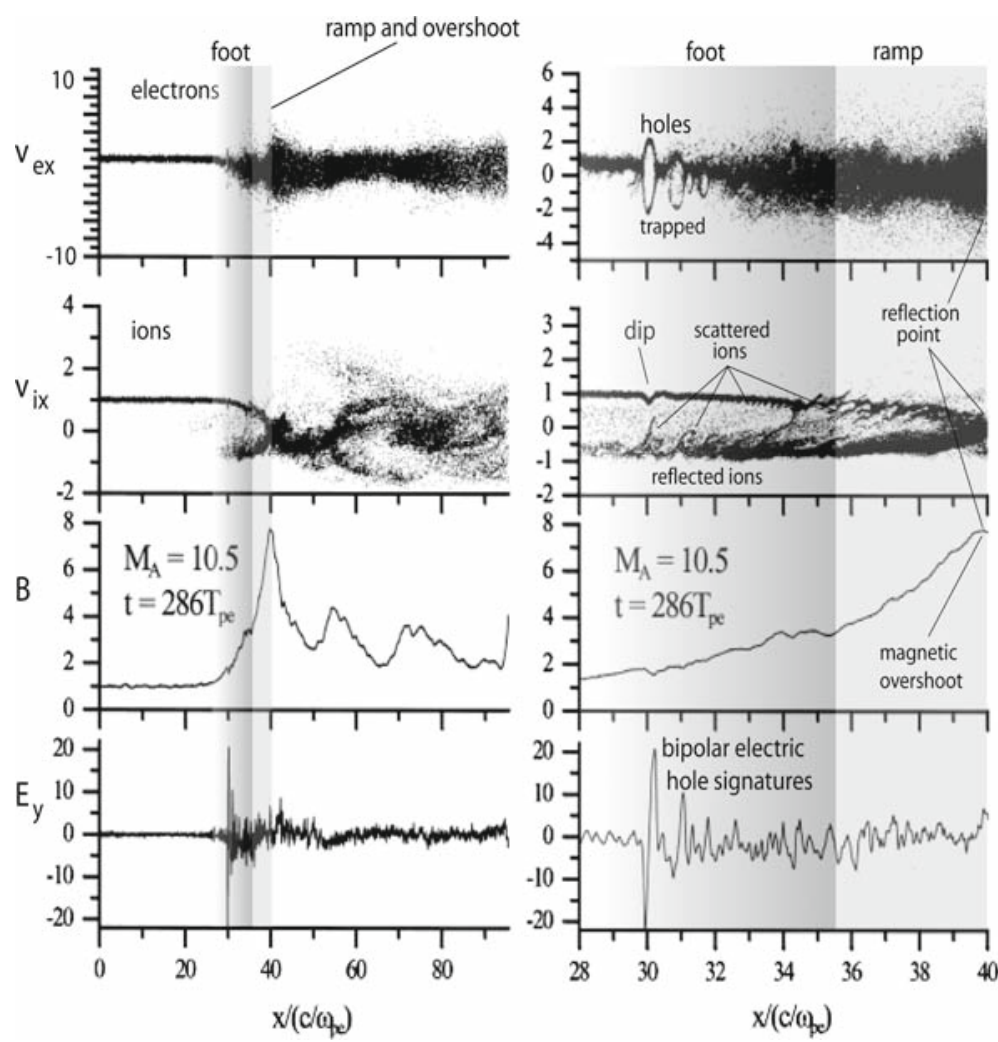

Fig. 191 D-PIC simulations $\left(m_{i} / m_{e}=20, \mathscr{M}_{A}=10.5, \theta_{B n}=90^{\circ}\right)$ resolving the electron scales (after Shimada and Hoshino 2000). Left: Electron/ion phase spaces, magnetic field, electric field. Second panel: ion reflection and foot formation. Bottom: Foot-electron heating in large electric field amplitudes. Right: Expanded view of shaded regions. Electron heating related to hole formation. Three Buneman holes form with trapped electrons. Second panel: Ion retardation in interaction with holes due to retarding electric potential (lowest panel) in the overshoot. Ion distribution is highly structured due to interaction with many small-scale electron holes

An electron hole, once evolved, distorts the ion and electron velocities in such a way that the velocity difference can increase nonlinearly and cause the generation of secondary vortices yielding excessive electron heating (Shimada and Hoshino 2000, 2005). The result is the generation of an extended electron tail on the electron distribution (Fig. 20 in a log-lin representation). When plotting the data on a log-log scale (not shown) one realises that the newly produced tail of the electron distribution has a power law slope $F(\epsilon) \propto \epsilon^{-\alpha}$, notably with power $\alpha \approx 1.7$, close to the marginally flattest power $\alpha=\frac{3}{2}$, below which an infinitely extended power law energy distribution can exist. The effect does not occur for small Mach numbers, too small for the BTS instability to be excited. However, once excited, the heating increases strongly with $\mathscr{M}_{A}$. Over the range $5<\mathscr{M}_{A}<20$ the increase in electron temperature (electron energy in the tail of the distribution) is a factor of 40-50, which demonstrates the strong non-collisional but anomalous transfer of flow energy into electron energy via the two-stream instability. 

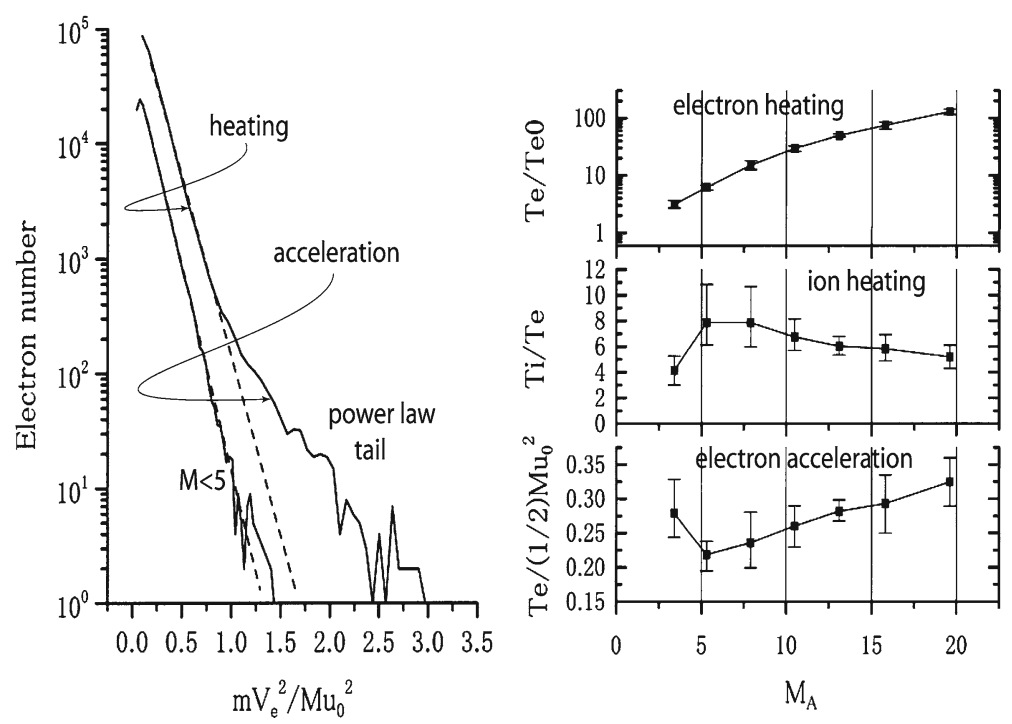

Fig. 20 Left: Heating and energetic tail formation in shock-electron distribution from Buneman electron hole interaction at $\mathscr{M}_{A}>5$. The tail has power law shape $F \epsilon \propto \epsilon^{-\alpha}$, with $\alpha \approx 1$.7. corresponding to marginal flatness $\alpha=\frac{3}{2}$. Right: Evolution of average temperatures electron temperature ratio as function of $\mathscr{M}_{A}$ (after Shimada and Hoshino 2000). All quantities are in computational units

Recently, the Polar satellite, when crossing the quasi-perpendicular Earth's Bow Shock, provided in situ measurements of very strong localised electric fields that exist on scales $\lesssim \lambda_{e}=c / \omega_{p e}$ and reach values of $\lesssim 100 \mathrm{mV} \mathrm{m}^{-1}$ parallel and $\lesssim$ $600 \mathrm{mV} \mathrm{m}^{-1}$ perpendicular to the magnetic field. These fields are related to the electron dynamics in the shock ramp. Presumably they play a substantial role in quasiperpendicular shock dynamics.

\subsubsection{Electron dynamics: modified two-stream effects}

The BTS instability works on scales $\lesssim \lambda_{e}=c / \omega_{p e}$. This condition is difficult to satisfy in quasi-perpendicular shocks. However, here other instabilities can evolve which are relatives of the BTS instability.

The condition in which there is no current flowing in the shock normal direction during foot formation and reflection of ions at the shock requires that the electron inflow from upstream be decelerated when entering the foot region. This causes a difference in the ion and electron inflow velocities. In a quasi-perpendicular shock the wave vector $\mathbf{k}=\left(k_{\|}, \mathbf{k}_{\perp}\right)$ is allowed to have a component $k_{\|}$along the magnetic field. The velocity difference between ions and electrons can then excite the MTSI, a modification of the Buneman instability acting in the direction perpendicular to the magnetic field. This instability is electromagnetic, coupling the BTS instability to the whistler mode. The waves generated propagate on the whistler mode branch with frequency $\omega_{\mathrm{mtsi}} \sim \omega_{\mathrm{lh}} \ll \omega_{c e}, \omega_{p e}$, close to the lower-hybrid frequency, but far below both the electron cyclotron and electron plasma frequencies. These waves are capable 


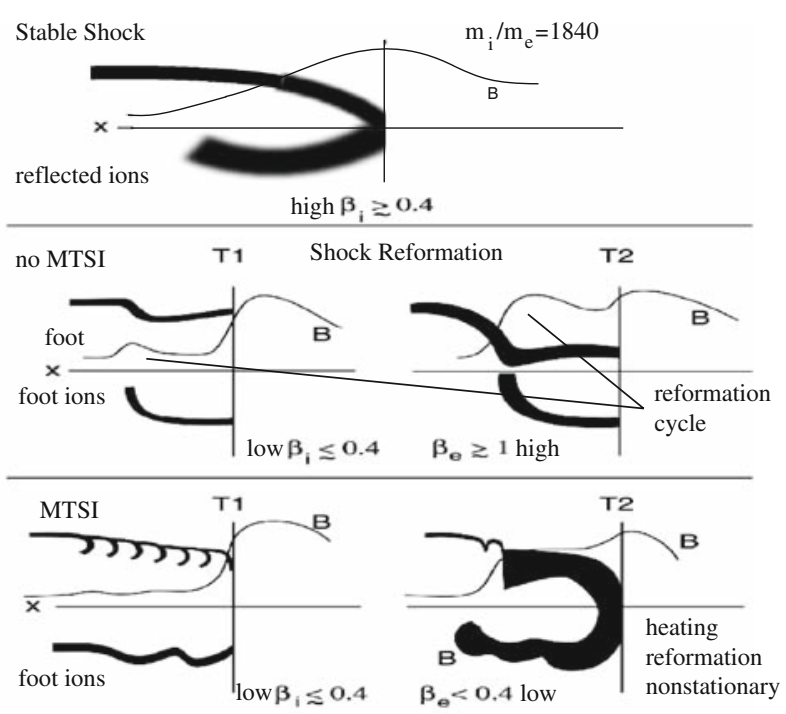

Fig. 21 Schematic of the dependence of the shock structure on the combinations of $\beta_{i}, \beta_{e}$ for quasi-perpendicular supercritical but non-whistler shocks. For large $\beta_{i}$ the shock is stable even though ions are reflected. At small $\beta_{i}$, large $\beta_{e}$ the shock reforms due to accumulation of ions at the edge of the foot forming in reformation cycle. For small $\beta$ the MTSI evolves in the foot, strong heating and complicated dynamics evolves due to nonlinear interaction, heating and hole-vortex formation (after Scholer and Matsukiyo 2004)

of modifying the shock profile when being swept downstream towards the shock ramp. Their obliqueness generates a magnetic field-aligned wave electric field component which accelerates, traps and eventually pre-heats the electrons in the shock foot along the magnetic field.

The transition from Buneman to MTSIs as a function of mass ratio $m_{i} / m_{e}$ and for various $\beta_{i}, \beta_{e}$ has been investigated (Scholer and Matsukiyo 2004) in the regime where no upstream standing whistlers exist, i.e. above the critical whistler Mach number $\mathscr{M}_{A}>\mathscr{M}_{\mathrm{wh}}$. This investigation is restricted to $\mathbf{k}$-vectors being strictly perpendicular to the shock along the shock normal and for one dimension only, excluding waves along the inclined magnetic field in the inclined direction. For mass ratios $m_{i} / m_{e} \lesssim 400$ no MTSIs occur in this case. The electron dynamics and the shock behaviour are determined exclusively by the two-stream instability unless the electron temperature is too large, inhibiting its growth, in which case an ion-acoustic instability could set in. For the realistic mass ratio $m_{i} / m_{e} \lesssim 1,840$ the Buneman two-stream instability disappears. Instead, the MTSI takes over which is strong enough to completely determine the behaviour of the electrons (Fig. 21).

The evolution of the MTS-waves for realistic mass ratio simulations is shown in Fig. 22 for three instants of time. The wave spectrum has been determined in the shaded area. Large amplitude waves of left-hand polarisation propagate toward the shock during this reformation cycle. These waves are related to the electron dynamics. They are excited by the MTSI in the foot (top panel) in interaction between the retarded electrons and the fast ions. The simulations also show the evolution of large amplitude electron holes and ion holes (right lower panel). Such structures have been observed 


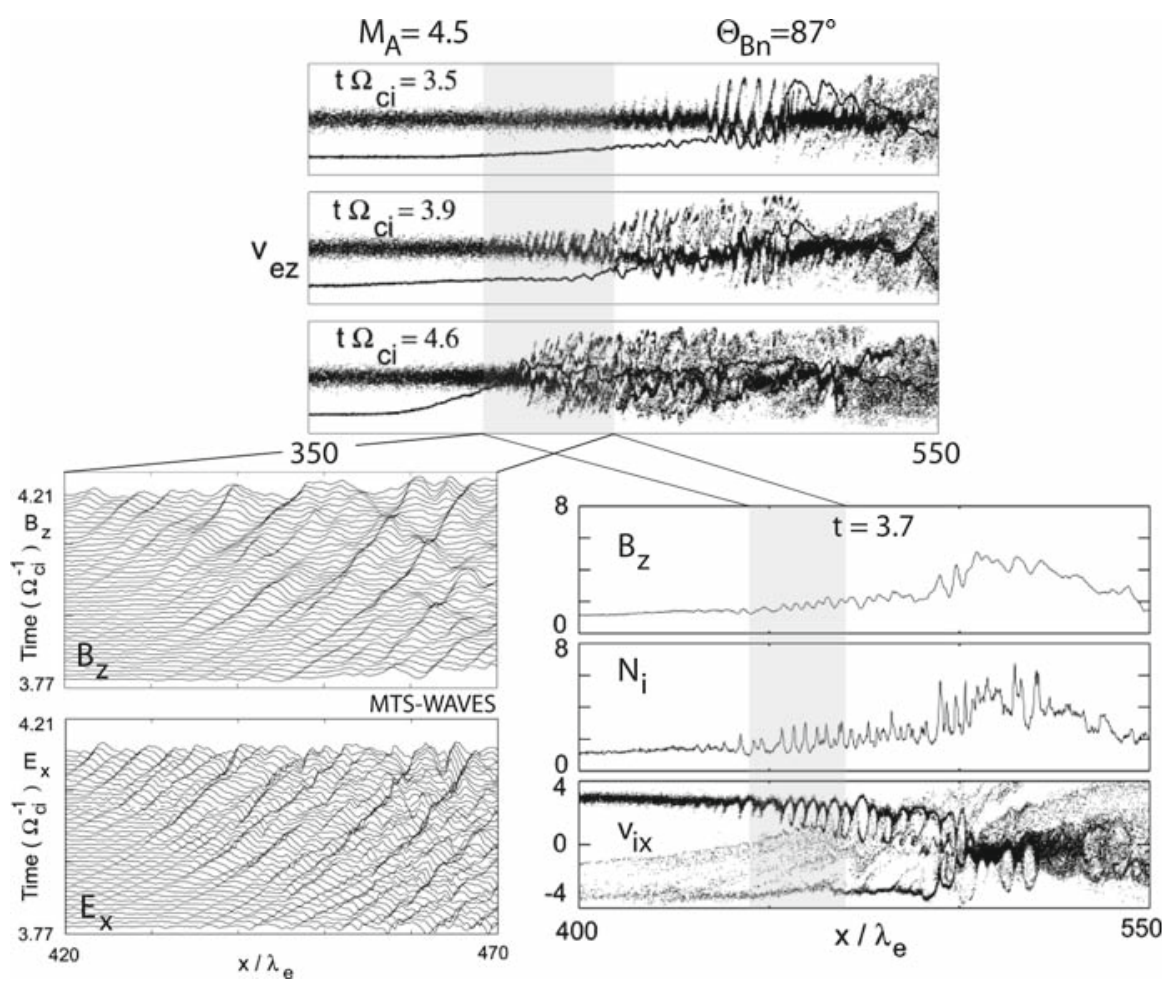

Fig. 22 Top: Electron phase space evolution showing the distortion of the electrons until thermalisation during the modified two-stream instability. The evolution of narrow electron holes can be seen of increasing amplitude (phase space width) the closer one comes to the shock ramp. Lower left: Magnetic and electric wave components of MTSI waves present in the grey shaded area in space during part of the time shown and are moving toward the shock ramp in the left-hand mode as discussed earlier. These waves steepen when reaching the shock front. Distortion of the ion distribution is the result as shown in Lower right. Large amplitude ion holes are formed as well (after Scholer and Matsukiyo 2004)

in the electric field in the quasi-perpendicular shock region (Bale et al. 2002; Pickett et al. 2004; Balikhin et al. 2005; Hull et al. 2006; Hobara et al. 2008) with differing interpretations. From the simulations it is concluded that both kinds of holes/solitary structures are excited near a quasi-perpendicular shock on similar scales while being related to the combined electron and ion dynamics. They cause reformation of the shock, but in a different way than it is caused for low mass ratio by the Bunemaninstability. There, reformation was the result of accumulation of ions at the upstream edge of the foot, while here it is caused by participation of the foot ions in the MTSI all over the foot and particularly close to the shock ramp, and presumably also at the ramp itself. Phase mixing of the ions leads to bulk thermalisation and formation of a hot retarded ion component in the foot region, which has similar properties to the downstream population and, when sufficiently compressed, takes over the role of the shock ramp. This can be seen from the lower right part of Fig. 22, which is a snapshot at time $t \omega_{c i}=3.7$ showing the magnetic profile, the density profile with its strong distortions, and the evolution of the ion distribution which evolves into large thermalised 

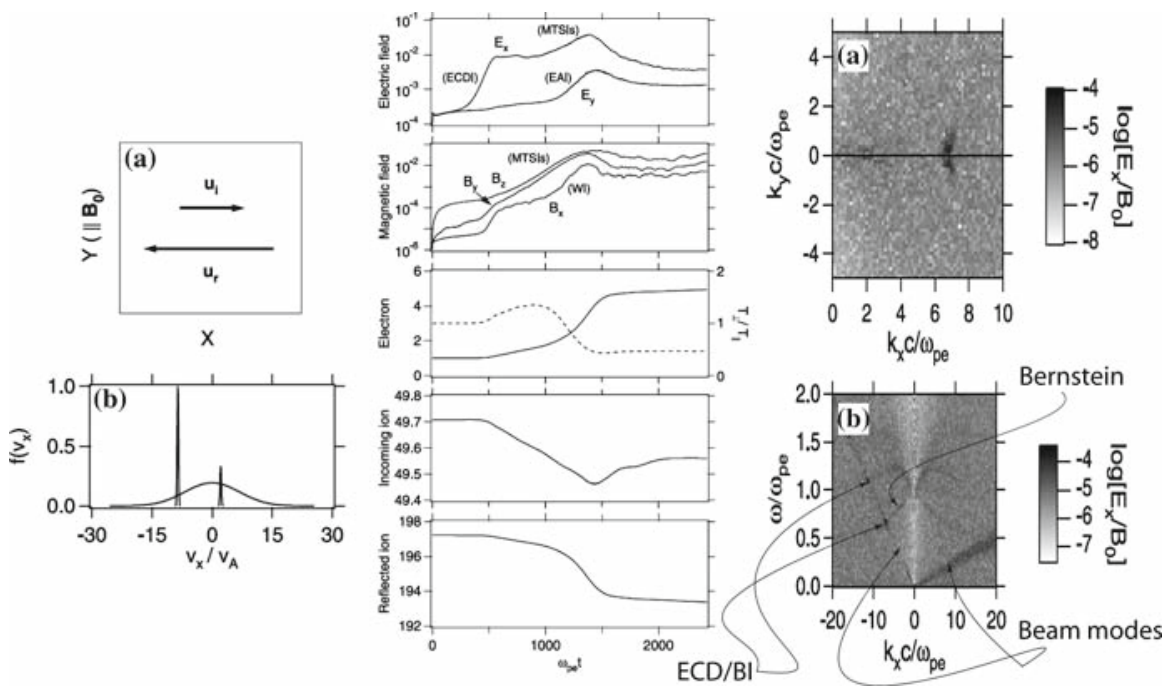

Fig. 23 Left: The phase space distribution set-up for the simulation. The original magnetic field is in $z$ direction. The upper panel shows the incoming and reflected ion beams. The lower panel shows the two cold ion distributions, incoming and reflected, and the hot electron distribution, shifted slightly in order to satisfy the zero-current condition in shock-normal direction. Centre: Time histories of the energy densities of the simulation quantities: electric and magnetic wave fields, electrons and the two ion components. Right: Wave power spectra in $\mathbf{k}$-space at early times $t \omega_{p e}<404.8$ showing the excited power in the Buneman mode in the upper panel. The lower panel shows the dispersion relation. The two straight lines correspond to the damped beam modes of the reflected (negative slope) and direct (positive slope) ion beams. The enhanced power in the two dark spots is due to the ECD-instability, which is the Buneman mode which excited under these early conditions in the simulation as the interaction between the reflected ion beam mode and the first and second Bernstein harmonic waves (after Matsukiyo and Scholer 2003, courtesy American Geophysical Union)

vortices towards the front of the shock (note that the shading indicates here also the spatial domain where the wave spectra have been taken).

\subsubsection{Modified two-stream instability: tailored simulations}

Figure 23 in its left-hand parts shows the set-up of the two-dimensional simulation and the resulting time histories of fields and particles. The incoming and reflected ion velocities are shown for time zero in the $(x, y)$-plane where the coordinate $y$ is about parallel to the magnetic field. The phase space at time zero contains the three distributions of inflow, reflected ions and hot incoming electrons. The slight displacement between the latter and the incoming ions accounts for zero normal current flow in the presence of reflected ions. Clearly this configuration is unstable, causing instabilities between the ion beams and electrons (in addition to the slowly growing ion-ion instabilities discussed earlier). The basic physics of the instability can be readily identified from the time histories of the fields and particles in the middle of Fig. 24. The first exponential growth phase of the $E_{x}$-component for times $\omega_{p e} t<500$ is due to the Electron-Cyclotron-Drift instability (ECDI). This instability is driven by the ion beam when it interacts with obliquely propagating electron-Bernstein waves 

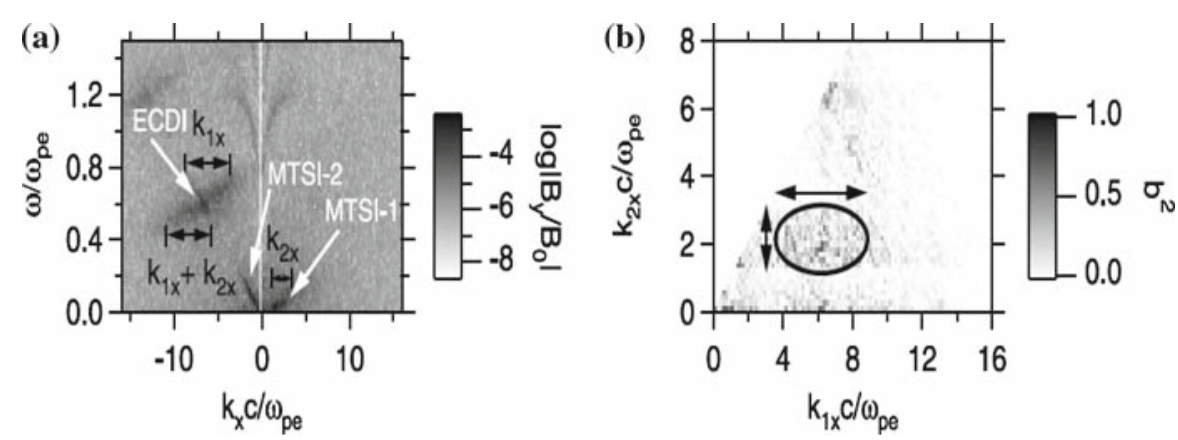

Fig. 24 Top: The dispersion relation for the time interval 607.2 $<t \omega_{p e}<1,011.9$ showing the ECDI (Buneman mode), the original MTI-1 and the secondary MTSI-2 which is generated by wave-wave interaction. The corresponding reaction in $k_{x}$ numbers is indicated for the waves which participate in the three-wave process. Bottom: The power spectral density in the $\left(k_{x}, k_{y}\right)$-plane. The ellipse indicates the wave numbers that contribute to the wave-wave interaction of the MTSI-1 and ECDI (after Matsukiyo and Scholer 2003, courtesy American Geophysical Union)

(electron-cyclotron waves). In the present case it is identical with the Buneman instability (BI), which for the given set-up is initially unstable (see the bulk velocity difference between ions and electrons on the left) due to the interaction of the ion beam mode with the lowest order electron-cyclotron mode. Initially there is also some growth in the magnetic field, which is strongest in $B_{z}$ and much weaker in $B_{y}$ and $B_{x}$. However, until the MTSI sets on the magnetic field energy does not grow substantially. This changes with onset of the MTSI when all components increase with $B_{y}, B_{z}$ dominating and being of equal intensity, showing that due to the magnetic wave field of the MTSI the instantaneous magnetic field develops a transverse out-of-coplanarity component.

\subsubsection{MTSI and Weibel effects}

Under the simulation conditions, the growth rate of the MTSI is initially small. The ECDI still dominates in the flat regime until the MTSI takes over, causing further growth of the already large amplitude electric field fluctuations. This stage after $\omega_{p e} t>10^{3}$ is characterised by a growth phase in $E_{y}$ (due to the electron acoustic instability EAI which can be excited in presence of a cold and a hot electron component) and, surprisingly, the normal component $B_{x}$. This latter component is caused by the Weibel instability (WI) when a substantial anisotropy has been generated. Such an anisotropy exists for the ions, in fact, in our case as they propagate solely in $\pm x$ direction at grossly different speeds. The growth rate of the instability for $\omega_{c e}=0$ is $\gamma_{\mathrm{WI}}=\left(V_{i} / \lambda_{i}\right)\left[1+\left(k \lambda_{e}\right)^{-2}\right]^{-\frac{1}{2}}$ (Weibel 1959). When the magnetic field is not neglected but the ions are taken as non-magnetised, as is the case in the shock foot, then $\gamma_{\text {WI }}=\left(V_{i} / \lambda_{i}\right)\left[1+\left(k \lambda_{i}\right)^{-2}\right]^{-\frac{1}{2}}$. At short wavelengths the growth rate of this instability can be quite large. Its maximum is assumed for $k \lambda_{i} \gg 1$ when it becomes the order of $\left(\gamma_{\mathrm{wI}} / \omega_{c i}\right)_{\max } \sim V_{i} / V_{A} \simeq \mathscr{M}_{A}$. At the expected wavenumber $k \lambda_{i} \sim 1$ is just a factor $\sqrt{2}$ smaller than its maximum value and decreases rapidly towards longer wavelengths. 
The simulations show the presence of $B_{x} \neq 0$, suggesting that the magnetic field becomes three-dimensional, since the Weibel instability has zero frequency and thus produces a steady normal field component. One may thus expect that, under astrophysical conditions of very large Mach numbers, magnetic fields will be self-consistently generated by the Weibel instability in the shock. In this case the field becomes necessarily non-coplanar, and small-scale stationary magnetic structures appear in the shock foot and ramp. Still, this is a little speculative. However, if the Weibel instability exists, it will generate many small-scale magnetic structures. Their presence leads to a wealth of secondary effects such as reconnection, dissipation, heating and particle acceleration.

The right outermost part of the figure shows two power spectra of the electric field in $\left(k_{x}, k_{y}\right)$-space at times $t \omega_{p e}=253$ (top) and $t \omega_{p e}<404.8$ (bottom). In the top panel, the power of the waves concentrates at $\left(k_{x} \lambda_{e}, k_{y} \lambda_{e}\right)=(6.8,0)$. These waves propagate nearly perpendicular to the ambient magnetic field. The lower panel shows the dispersion relation $\omega\left(k_{x}\right)$ for these waves in a grey scale representation. The two straight dark lines with negative and positive slopes belong to the damped ion beam modes for the reflected (negative slope) and incoming (positive slope) ion beams.

There are two dark specks on the reflected beam mode where the intensity of the electric field (which is shown here only) is enhanced. These specks are separated by about the electron cyclotron frequency. They belong to the crossings of the reflected ion beam mode and the two lowest harmonics of the electron Bernstein modes which, in this case, is the Buneman instability (BI). It is no surprise to find it in the early stage also in two dimensions, since the initial situation is still close to one-dimensional. However, here the two-stream instability results from the large difference in bulk speeds between electrons and reflected ions. In the later stages, as the existence of the electromagnetic left-hand polarised negative helicity waves in Fig. 22 confirms, the ECDI/Buneman mode is replaced by the MTS-instability which generates oblique, nearly perpendicularly propagating large amplitude electromagnetic waves, which form hole structures and heat the plasma.

Figure 24 shows the next time slot in the presentation of the dispersion relation (left). At this time the waves have reached large amplitudes, large enough to cause various interactions among the waves which react on the wave and particle distributions and, in addition, cause nonlinearity of the plasma state at wave saturation. The ECDI forms as a broad spot in the $(\omega, k)$-domain. The MTSI is the short nearly straight line at low frequencies and small positive $k_{x}$ (indicated as MTSI-1 in the figure). In the wave spectrogram these waves move towards the shock ramp. This means their slope is positive in the dispersiogram! (Fig. 25).

In addition to the MTS-instability a secondary MTS can be identified in the simulations which grows at later times and is caused by a three-wave-beating interaction between waves in a way similar to that predicted for magnetosonic waves by Sagdeev (1966). The magnetic field contains signatures of both the original MTS (MTS-1) and the secondary (MTS-2), thus exhibiting a more irregular structure than the electric field. Here, also the small-scale structures of the Weibel instability do contribute. We have noted their effect above, but it would be rather difficult to extract them from the figure as they should appear as stationary vortices, which are convected downstream towards the shock front with the speed of the average bulk flow. Their dynamics 

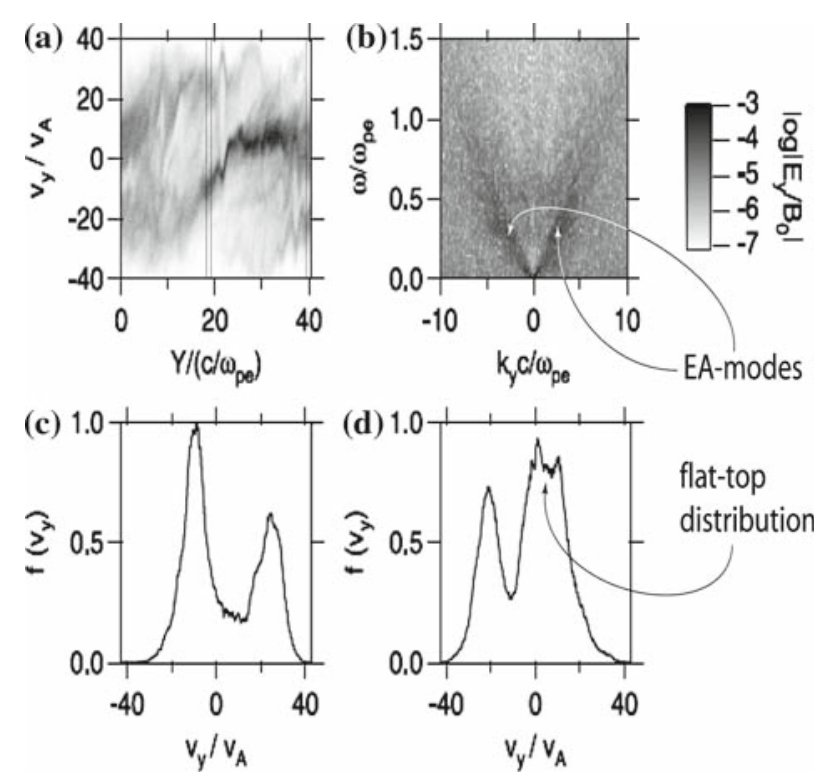

Fig. 25 Top: Electron phase space (left) at $19.4<x / \lambda_{e}<21$, dispersion relation (right) $\omega\left(k_{y}\right)$ for $910.7<t \omega_{p e}<1,315.5$. EA-waves with strictly linear dispersion and frequency below $\omega_{e a} \lesssim \omega_{p e}$ propagating in both directions generated in two-electron component structure in distribution function (below), being responsible for fine-structuring (upper left panel) in $v_{y}$ versus $y$. Electron trapping/scattering on small scales seen. Bottom: Distributions at $17.6<y / \lambda_{e}<19$ and $39.1<y / \lambda_{e}<40.5$ at $t=1,000$ (vertical lines in upper left), showing large electron hole distribution generated by MTSI and smaller substructures. (after Matsukiyo and Scholer 2003, courtesy American Geophysical Union)

remains unresolved, i.e. it is not clear what will happen to them when encountering the shock front. One possibility is that they accumulate there and contribute to a noncoplanar magnetic component. Nevertheless, the possibility for the Weibel instability to evolve in supercritical quasi-perpendicular shocks is of interest as Weibel vortices should cause an irregular fine structuring of the magnetic field in the shock ramp transition. This has important consequences for particle dynamics, trapping, scattering, reflecting and acceleration of particles from the shock front. It could, moreover, also lead to small scale reconnection in the shock front, which so far has not been believed to exist in shocks, including the various side-effects of reconnection. Weibel vortices could also pass across the shock into the downstream region where they contribute to the downstream magnetic turbulence and would occur as magnetic nulls or holes for which the shock is the source.

\subsection{Plasma waves and radiation in quasi-perpendicular shocks: observations}

From an astrophysical point of view the question arises, which of the above effects can be inferred from the remote observation of radiation? We will return to a brief discussion of this question in the next Summary section. However, in order to get some feeling of what kind of in situ signature the waves that are produced in shocks leave, 

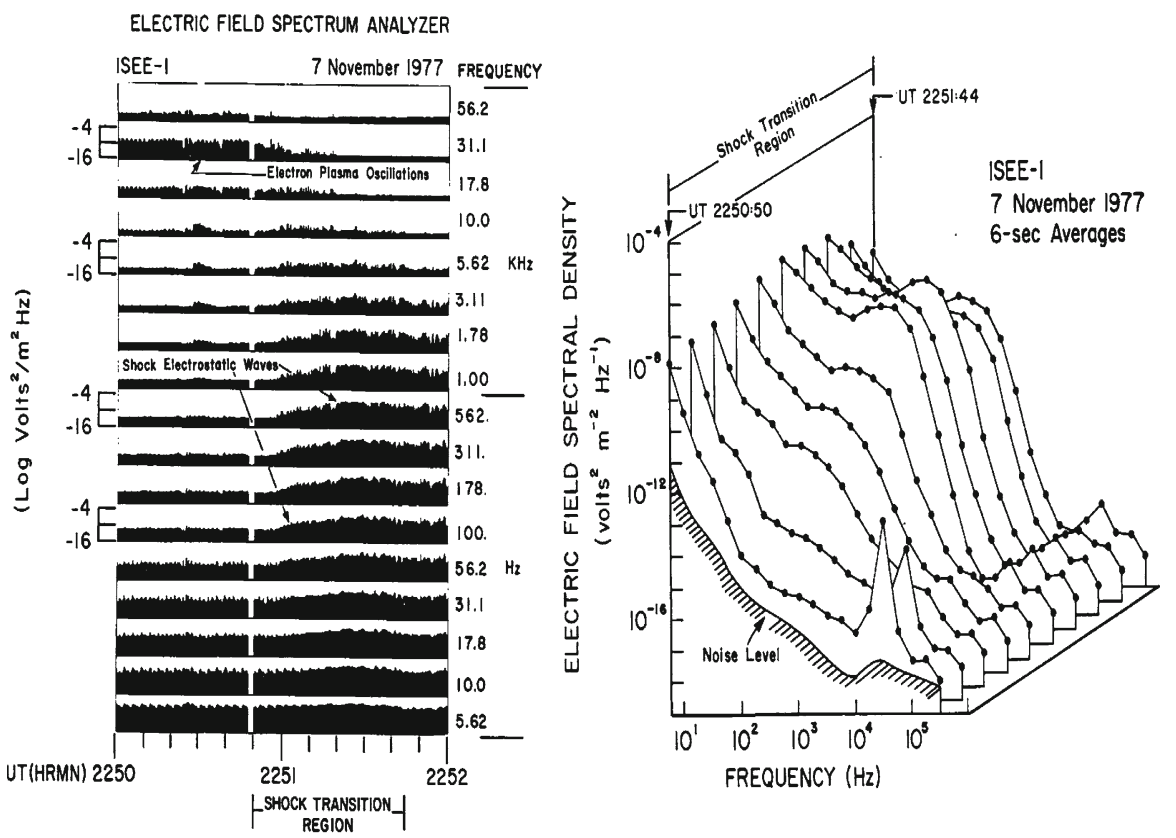

Fig. 26 Electric wave spectra measured during spacecraft crossing of an interplanetary shock (after Gurnett et al. 1979, courtesy American Geophysical Union). Left: Power spectra (in $\mathrm{V}^{2} / \mathrm{m}^{2} \mathrm{~Hz}$ ) with respect to time in a number of frequency channels. The spacecraft approaches the shock from left and crosses over it. The increase in power is well documented from low to high frequencies when coming into the shock transition region. Right: A sequence of shock electric spectra during this crossing given as power spectral density with respect to frequency. The dramatic increase of the low frequency wave power is seen when the spacecraft approaches and crosses over the shock. Behind the shock the power remains high but lower than in the transition region. The Bump around a few $100 \mathrm{~Hz}$ is the most interesting from the point of view of instability. These waves are excited by electron-ion instabilities discussed in the next section

Fig. 26 on its left shows two minutes of electric wave field spectra detected during a spacecraft crossing of Earth's bow shock. The spacecraft approaches the shock from upstream. In the upstream plasma it records general low frequency turbulence at frequency $\lesssim 1 \mathrm{kHz}$. Centred at $\simeq 31 \mathrm{kHz}$, the highly enhanced wave intensity shows the presence of intense electron plasma waves with frequency $\omega \simeq \omega_{p e}$. They are generated by the electron beam, which has been accelerated in the quasi-perpendicular shock and reaches the spacecraft along the tangential magnetic field line that connects the spacecraft with the shock. Some signatures of these highly fluctuating waves are also seen near $18 \mathrm{kHz}$, and due to nonlinear effects occasionally down to $1 \mathrm{kHz}$, as for instance at 2,250:30 UT. The electron plasma waves disappear when approaching the shock at 2,251 UT, when the shock turns up very intense broadband noise from $0-10 \mathrm{kHz}$. The sequence of spectra at the right shows the sharp plasma wave peak in the first few spectra and the gradual evolution of the broadband noise peaking around $0.5 \mathrm{kHz}$ related to the Buneman and MTSIs and the presence of localised electron holes in the shock. Radiation from the shock can be recognised only upstream in the $56 \mathrm{kHz}$ channel as second harmonic radiation at twice the electron plasma frequency 


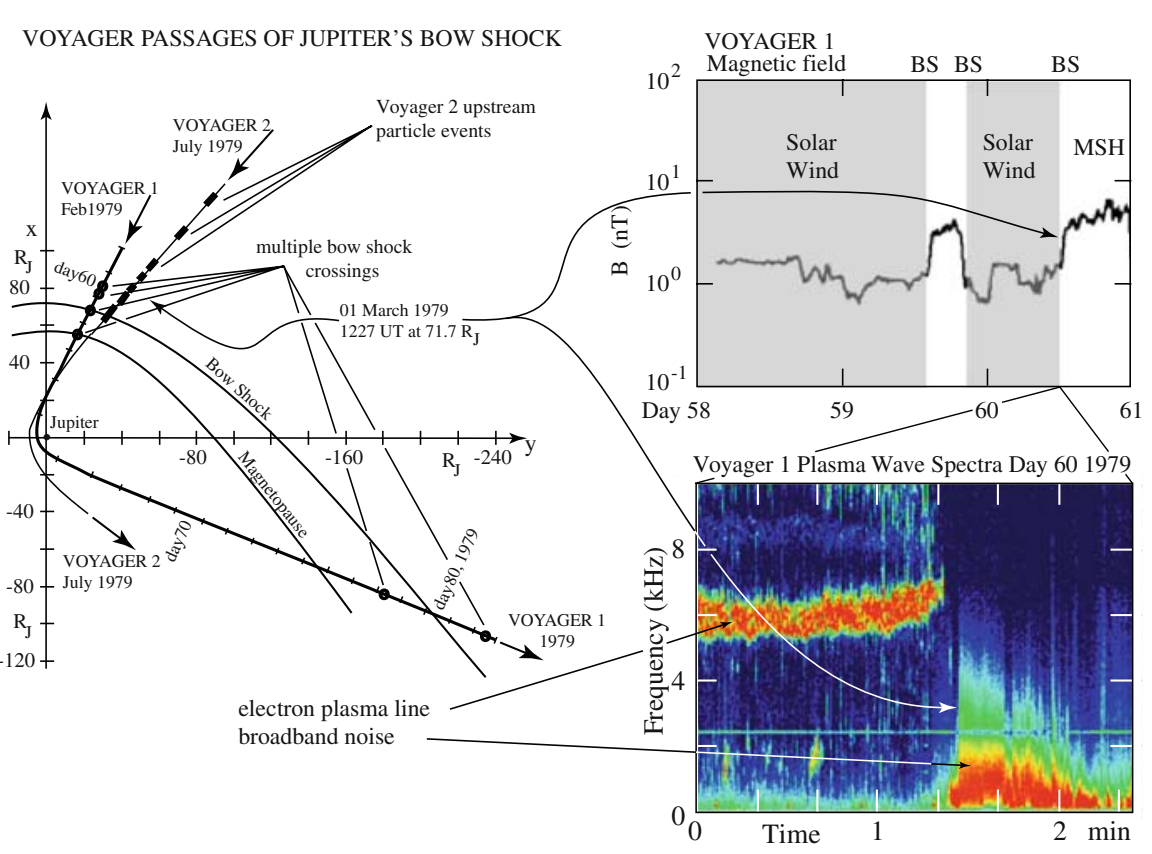

Fig. 27 Voyager 1 crossings of the Jovian bow shock. Left: Projected $(x, y)$-plane V-1/V-2 orbits and nominal bow shock and magnetopause ( $x$ towards sun). Numbers give days of 1979, dots indicate the multiple crossings (bow shock variability) (after Ness et al. 1979). Bars indicate upstream energetic particle events (after Krimigis et al. 1985). Right: Selected magnetic field modulus (top) and plasma wave spectra (bottom). $B$ increases (decreases) inbound (outbound) crossings. Plasma wave spectra are for the third crossing (red highest, black lowest intensity). Intense plasma frequency at $6 \mathrm{kHz}$ indicates electron foreshock. Passage of the shock occurs as a sharp cutoff of the plasma frequency and start of broadband low frequency noise (courtesy by D.A. Gurnett, U Iowa)

$\omega \simeq 2 \omega_{p e}$ that is generated locally and remotely by the beam-excited electron plasma waves. Fundamental radiation at $\omega_{p e}$ and radiation from the shock transition cannot be distinguished in these in situ observations from locally excited waves. Nevertheless these observations prove that shocks are indeed sources of radio emission at the plasma frequency and its low harmonics. Mechanisms for generating this kind of radiation in non-relativistic supercritical collisionless shocks are well known. The favoured mechanism is the three-wave interaction process which can be symbolically written as $L+L^{\prime} \rightarrow T$, where $L, L^{\prime}$ are two longitudinal waves and $T$ is the radiated transverse wave. Another example, now taken from the Voyager 1 crossing of the Jupiter bow shock, is shown in the wave spectrogram of Fig. 27. The broad intense and highly fluctuating emission band around $\sim 6 \mathrm{kHz}$ are the electron plasma waves upstream of Jupiter's bow shock again generated by the shock-accelerated electron beam. These waves disappear at shock approach, becoming replaced by the lower frequency broadband noise in the shock transition. Emitted radiation is detected in two narrow bands upstream of the shock, a weak emission at $\sim 9 \mathrm{kHz}$ of unidentified origin, and a stronger emission above $10 \mathrm{kHz}$, of which only the lower end is seen due to instrumental cut-off and which is the harmonic radiation centred at $12 \mathrm{kHz}$ coming from the plasma 


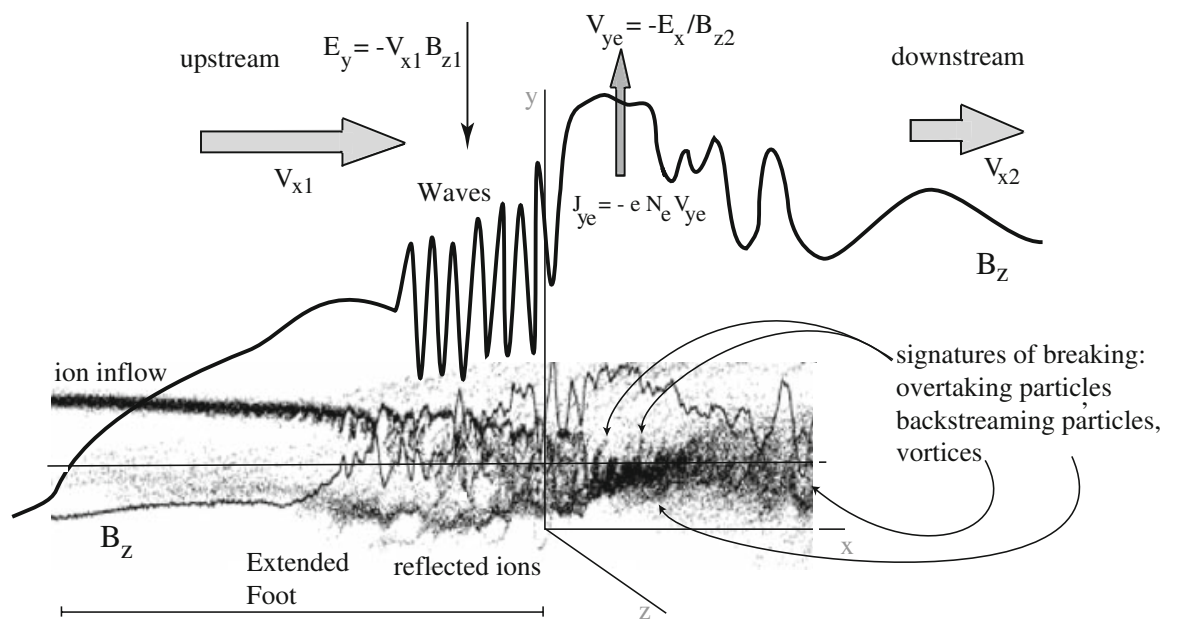

Fig. 28 Schematic of the profile of a highly supercritical quasi-perpendicular shock with waves just before shock reformation and signatures of beginning wave breaking. The sketch has been completed with a copy of the ion phase space from the simulations of Matsukiyo and Scholer (2006b) showing the structure of the ions in the ramp with the signatures of overtaking ions and backstreaming ions as well as ion vortices, all an indication of the onset of breaking in the particle component

waves at $6 \mathrm{kHz}$. Again, any fundamental emission at $\omega_{p e}$ from the plasma wave band is hidden and cannot be distinguished from the plasma waves. However, the $9 \mathrm{kHz}$ band is new and might be related to emission from the shock transition known from solar type II bursts as the backbone emission. It corresponds to fundamental emission from a shock plasma of density $N \sim 1 \mathrm{~cm}^{-3}$ from a region in the shock front roughly 2.2 times denser than upstream and being located right in the ramp. Such an emission should be caused by the electron holes (cf., e.g., Treumann 2006) which are expected to exist in the shock ramp.

\subsection{Summary of quasi-perpendicular shocks}

A graphical summary of a quasi-perpendicular supercritical shock is given in Fig. 28. The magnetic profile shows the extended shock foot which, close to the shock ramp, is quite irregular, being modulated by low frequency propagating waves. The ramp is the transition to a smoother shock overshoot profile and from there to the downstream region. The ion phase space exhibits the incoming and reflected ion beams. In the foot, the former is retarded, showing the signatures of the slowed down ions which have been scattered in the wave field inside the foot. When interacting with the reflected beam and the electron-generated waves, a sequence of phase-space structures is formed which, closer to the shock ramp, evolve into phase space holes. The shock ramp is the location of the ultimate ion reflection and heating of the bulk distribution. Signatures of wave breaking are seen in the overtaking particles. The entire transition region, including the foot and downstream region, contains a diffuse hot energetic ion component that has been accelerated in the shock. The plasma state just downstream 
of the shock is nothing else but the collection of the old shock ramps which have been left over from former reformation cycles and, relative to the shock frame, move in the direction downstream of the shock. The simulations show that, in more than one dimension, the shock front is far from being a plane surface. It exhibits a strong variability in time and space. Some of this variability can be explained as surface waves propagating along the shock front. These surface waves might be driven by the reflected ion current-flow along the surface in similarity to a Kelvin-Helmholtz instability. At the time of writing it remains unclear whether or not shock breaking can be realised at non-relativistic Mach numbers. Magnetic field lines cannot break-off; they can only kink and must remain connected. In order for the magnetic field to contribute to breaking, the field must evolve into vortices and thus undergo reconnection on a broad range of scales. This can happen when the Weibel instability generates transverse magnetic field vortices which cause tearing modes to evolve in the shock. At low Mach numbers neither of these effects is expected. However, when the shocks become relativistic, the Weibel instability becomes strong enough. It is thus possible that large Mach number relativistic shocks exhibit magnetic breaking as an additional mechanism of dissipation and thermalisation.

At the low Mach numbers considered in this paper, any breaking that is going on takes place only in the particle population and requires non-adiabatic effects. It will be related to vortices. In this sense the appearance of phase space vortices at high Mach numbers indicates a tendency for shock breaking. The lower two panels in Fig. 18 can be interpreted as breaking and overturning of the quasi-perpendicular $\left(\theta_{B n}=87^{\circ}\right)$ realistic mass-ratio supercritical shock. During the phase before reformation (third panel from top), the magnetic field behaves irregularly, and both the incoming and reflected beams indicate the emergence of vortices before reaching the reflection point (at $\Delta x \sim 140 \lambda_{e}$ ). Behind the reflection point, the ion velocity shows formation of bursts of ions which run away in forward direction, which is just what we expect when breaking occurs.

\section{Quasi-parallel supercritical shocks}

\subsection{Introduction}

Because of their large extension, astrophysical shocks are believed to be mostly quasiperpendicular or even strictly perpendicular. In the environment of very massive objects with their strong magnetic fields, only parallel plasma flows are possible. Shock normals are along the magnetic field, $\theta_{B n}=0$, and the shocks should be genuinely parallel. This might be true for shocks near massive objects and in the jets emitted from them. Still, in the much weaker magnetic field environments of SNRs, in clusters of galaxies, galaxies, stellar winds and the interstellar medium, the assumption that astrophysical shocks are genuinely perpendicular is highly questionable. While the shock transition might be very extended-in quasi-perpendicular shocks it is extended mainly in the direction downstream of the shock ramp and foot-we have seen that the width of the shock ramp is only of the order of the ion inertial length $\lambda_{i}$, microscopically thin against any astrophysically resolvable length. Even 


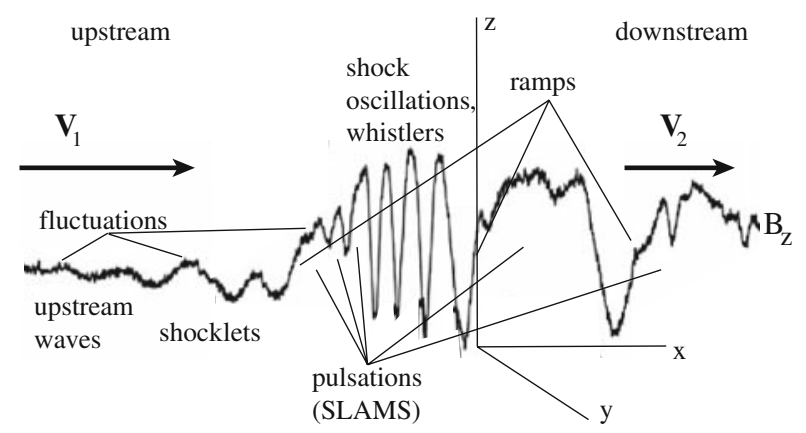

Fig. 29 Schematic one-dimensional profile taken along the nominal instantaneous shock normal of a supercritical quasi-parallel shock as seen in the magnetic field component $B_{z}$. This is the analogue to the quasi-perpendicular shock profile. It shows the main features in the vicinity of the quasi-parallel shock transition: the large amplitude upstream waves with the turbulent fluctuations on top of the waves, the formation of shocklets, i.e. steep flank formation on the waves exhibiting small-scale fluctuations on top of the wave, which act already like small shocks, very-large amplitude pulsations (magnetic pulsations or SLAMS) which turn out to be the building blocks of the shock, multiple shock-ramps at the leading edges of the pulsations belonging to diverse ramp-like steep transitions from upstream to downstream lacking a clear localisation of the shock transition and any attached phase-locked whistlers. (Note that the entire figure is, in fact, the shock transition, as on this scale no clear decision can be made where the shock ramp is located.) Not shown here are the out of plane oscillations of the magnetic field that accompany the waves. Also not shown is the particle phase space

when including the foot and shock, the scale is just of the order of (10-100) $\lambda_{i}$. The comparably enormous extension of the shock in a tangential direction is by no means plane and flat. It oscillates and is vulnerable to distortions in the flow speed, density and the magnetic field in the upstream shock environment. It is rippled on long scales $\sim\left(10^{2}-10^{3}\right) \lambda_{i}$. One thus expects that real astrophysical shocks are quasi-parallel at least as frequently as quasi-perpendicular. This conclusion is supported by the in situ observation of interplanetary shocks in the heliosphere and, in particular, by the Voyager crossings of the heliospheric termination shock. All these shocks are not of one kind of shock, but change along their surfaces according to the conditions in the interplanetary medium, the solar wind and the shock-driver flows.

Quasi-parallel shock physics is quite different from the physics of quasi-perpendicular shocks. The schematic of the magnetic profile of a quasi-parallel shock is shown in Fig. 29. In contrast to quasi-perpendicular shocks, the flow upstream of the shock is highly disturbed up to large distances from the shock. These disturbances grow in amplitude and shrink in length when approaching the shock-becoming pulsations (or SLAMS, which stands for Short Large Amplitude Magnetic Structures). Near the shock these pulsations are indistinguishable from the shock ramp. The magnetic shock transition is recognised from its broad width and the disappearance of the shorter scale fluctuations. Downstream, the shock the medium is highly disturbed. Structurally the main difference is that quasi-perpendicular shocks exhibit a sharp shock transition with a narrow foot region, while quasi-parallel shocks lack such a foot. Instead, the foot is replaced by a broad foreshock that is extended far into the upstream flow. While quasi-perpendicular shocks are narrow transitions, quasi-parallel shocks form broad extended and highly structured transition regions between the fast upstream and the 
slow downstream flow. The shock itself is found at the location of maximum thermalisation. Because of this spatial extension of the shock transition, from an astrophysical point of view one might expect that it is rather the quasi-parallel than quasi-perpendicular shocks which are subject to remote observation.

\subsection{The foreshock}

The physics of quasi-parallel shocks cannot be understood without reference to the foreshock. The foreshock is that part of the upstream shock region that is populated by shock-reflected particles. At a curved shock the foreshock starts on the shock surface at the location where the upstream magnetic field shock-normal angle exceeds $\theta_{B n} \gtrsim 45^{\circ} .{ }^{19}$ From that point on, reflected electrons and ions escape along the magnetic field in an upstream direction. Since electrons generally move at larger parallel velocity than ions, they are less vulnerable to convection of the upstream magnetic field line to which they are tied, and so there is generally a region closer to the foreshock-boundary magnetic field line where only upstream electrons are found. This region is approximately confined between the line that marks the electron foreshock boundary and the more inclined line that marks the ion foreshock boundary.

More schematically this is shown in a simplified version in Fig. 30 for the particular case in which the upstream magnetic field forms an angle of $45^{\circ}$ with the symmetry axis of the shock. In this case half of the shock is quasi-perpendicular and the other half is quasi-parallel. The figure also shows the directions of three shock normals, the narrow foot region in front of the quasi-perpendicular shock, and the two (electron and ion) foreshocks. Particles escape from the quasi-parallel shock along the upstream magnetic field. The magnetic field is convected toward the shock by the perpendicular upstream velocity component $\mathbf{V}_{\perp}$ as shown in the figure. This component adds to the velocity of the upstream particles leading to an inclined foreshock boundary. Since the ions have much smaller speed than the electrons, the ion foreshock boundary is more inclined than the electron foreshock boundary.

The properties of the ion foreshock are decisive in the process of formation of a quasi-parallel shock. On the other hand, it is the electron foreshock which provides the shock its visibility in radiation.

\subsubsection{Ion foreshock}

The shock-reflected ion component evolves across the ion foreshock from the ion foreshock boundary to the centre of the ion foreshock and from there towards the shock. The reflected ion component can be identified only along the ion foreshock boundary, where the reflected ions appear as a fast ion beam, the source of which can be traced back to the quasi-perpendicular part of the shock. Deeper in the foreshock the beam component becomes diffuse. In velocity space it forms a ring around the direction of

\footnotetext{
19 From this point of view the foreshock is the foot of the quasi-parallel supercritical shock. However, its large spatial extension provides it with new and completely different properties. It is therefore reasonable to consider it as a separate phenomenon that differs from the foot of a quasi-perpendicular shock, even though part of its particle population has its origin in the quasi-perpendicular section of the curved shock.
} 


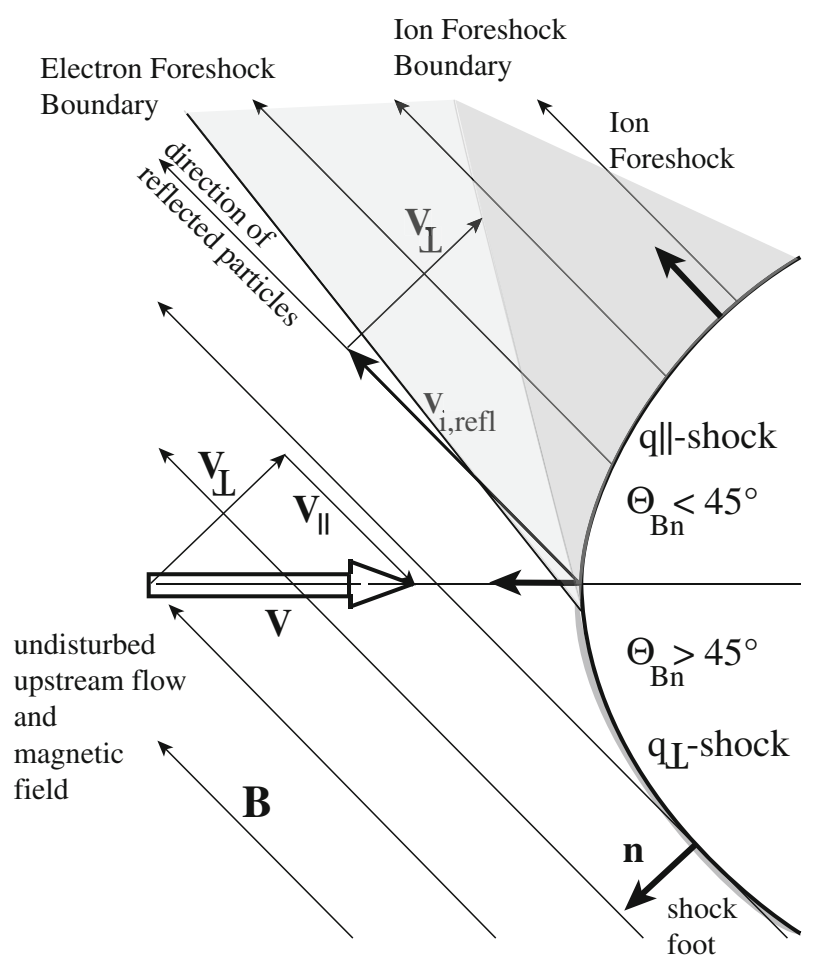

Fig. 30 Schematic of the relation between a curved shock and its foreshock in dependence on the direction of the upstream magnetic field $\mathbf{B}$, shock-normal $\mathbf{n}$, and shock-normal angle $\theta_{B n}$ for the special case when the magnetic field is inclined at $45^{\circ}$ with respect to the symmetry axis of the shock. In this case the upper half of the shock becomes quasi-parallel $\left(\theta_{B n}<45^{\circ}\right)$, the lower half is quasi-perpendicular $\left(\theta_{B n}>45^{\circ}\right)$. The velocity of reflected particles is along the magnetic field. However, seeing the flow the field-line to which they are attached displaces with perpendicular velocity. This velocity shifts the foreshock boundary toward the shock as shown for electrons (light shading) and ions (darker shading). The ion foreshock is closer to the shock because of the lower velocity of the ions than the electrons. For the electrons the displacement of the electron foreshock boundary is felt only at large distances from the shock

the solar wind. Extended quasi-parallel shocks are locally curved by surface waves and inhomogeneities in the upstream flow that are caused by the interaction of the flow with the shock particle component. It turns out that ions are reflected from the many quasi-perpendicular parts of the large-scale extended shock. In this case the many ion foreshocks overlap, and no reflected ion beam can be distinguished anymore. Instead, the entire extended average foreshock is filled with a diffuse reflected ion component.

Figure 31 gives an observational example of such a single reflected ion beam at Earth's bow shock that propagates upstream along the ion foreshock boundary away from the shock. The bulk flow is the narrow cold peak in the left central part of the figure displaced in a negative $v_{x}$-direction. The reflected beam is at higher energy, displaced in a $+v_{x}$-direction, streaming away from the shock and spreads in $-v_{y}$. It constitutes a gyrating bunch of ions. Deeper inside the foreshock (bottom panels) it forms a hot ring around the upstream flow. This ring is formed in the interaction 

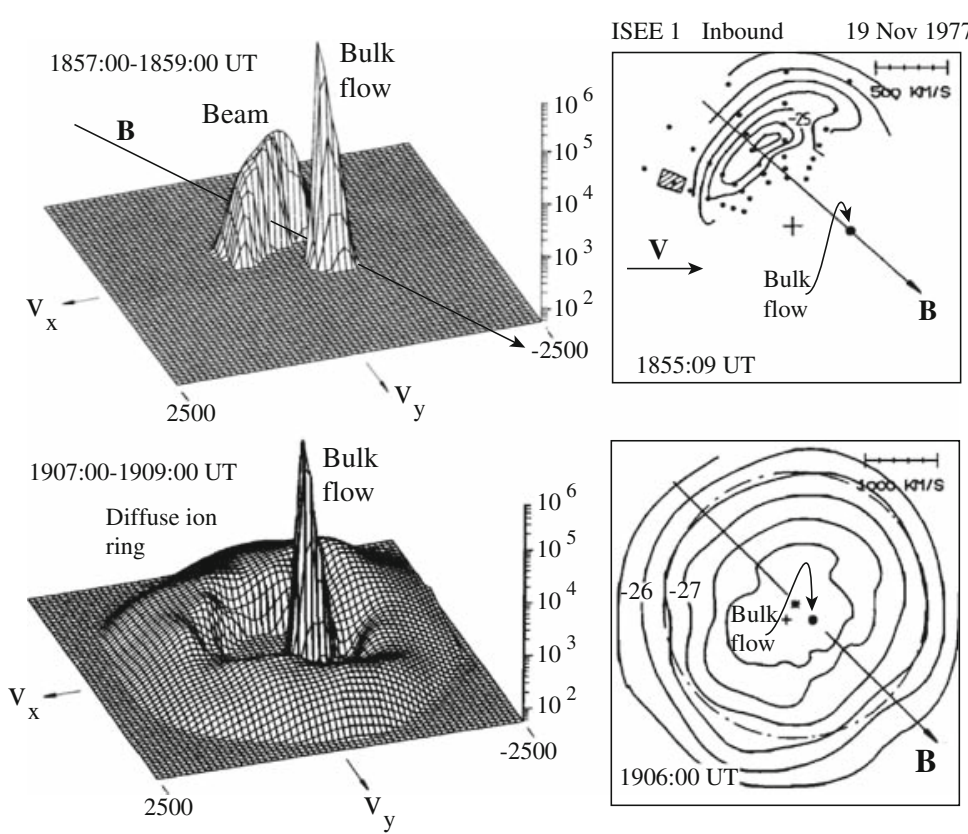

Fig. 31 Observation in situ a shock-reflected ion beam and its transformation into an ion ring. Left: Pseudothree-dimensional ion velocity-space profile in the $\left(v_{x}, v_{y}\right)$-plane showing the undisturbed cold (narrow) plasma inflow (negative $v_{x}$-direction), the fast warm (broad) reflected beam (positive $v_{x}$-direction) spreading in $v_{y}$ until forming the ring (Bottom). Velocities are in $\mathrm{km} \mathrm{s}^{-1}$. The scale on the right is count rates. Right: Contour plot of beam. The beam is centred on the magnetic field line that connects to the shock, is narrow along the field and 2-3 times as broad perpendicular to the field (data from Paschmann et al. 1981, courtesy American Geophysical Union). The cross is at zero velocity, the dot is the bulk flow centre. The $10^{-25} \mathrm{~s}^{3} \mathrm{~cm}^{-6}$ level flux contour has been marked

of the beam with self-generated upstream waves. The diffuse ion density decreases exponentially with distance from the shock as is shown in Fig. 32.

The spatial decay of the partial diffuse ion density $N_{i}(\mathscr{E}, z) \sim \exp [-z / L(\mathscr{E})]$ differs for particles of different energy $\mathscr{E}$. The e-folding distance $L(\mathscr{E}) \sim \mathscr{E}$ increases linearly with energy, suggesting that low energy particles are confined to the shock. The higher the ion energy, the deeper the ions penetrate into the upstream flow. In contrast to the ion beam, whose source is at the quasi-perpendicular/quasi-parallel shock boundary, the source of the diffuse ions is located at the quasi-parallel shock. These diffuse ions are thus different from the beam ions at the ion-foreshock boundary. In order to escape upstream, the diffuse ions must undergo a diffusion process along the magnetic field.

The e-folding distance for the diffuse ions is given by $L(\mathscr{E})=\kappa_{\|}(\mathscr{E}) / V_{1}$, with spatial diffusion coefficient $\kappa_{\|}(\mathscr{E})=\frac{1}{3} v \ell_{\|}(\mathscr{E})$, where $\ell_{\|}$is the diffusion length, and $v$ the particle velocity. From the balance between convective inflow and upstream diffusion, one writes

$$
\ell_{\|}(\mathscr{E})=3 L(\mathscr{E}) \sqrt{\mathscr{E} 1 / \mathscr{E}} \sim \sqrt{\mathscr{E} \cdot \mathscr{E}_{1}}
$$




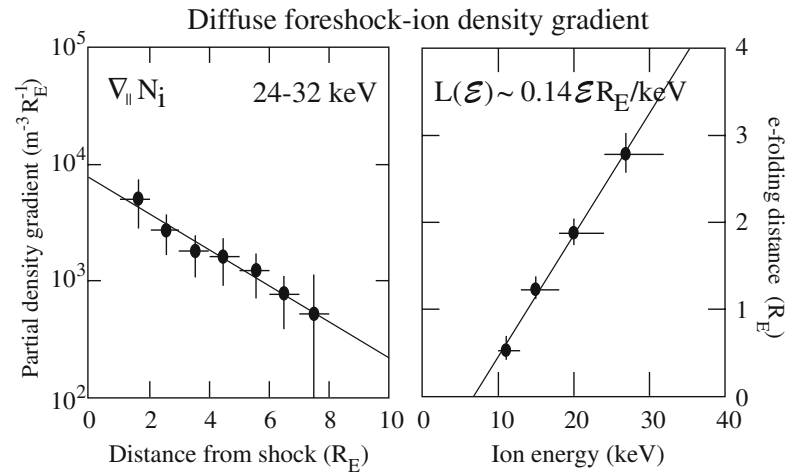

Fig. 32 Partial-density gradient and e-folding lengths of diffuse foreshock-ions component at tangential field line (data from Kis et al. 2004, courtesy American Geophysical Union). Left: Parallel diffuse-ion density as function of shock distance showing exponential decay suggesting energy dependent diffusion. Right: The e-folding distance of diffuse ions as function of energy being proportional to ion energy

with upstream flow energy $\mathscr{E}_{1}$. In the solar wind the flow energy is a few keV, and a 20 $\mathrm{keV}$ diffuse ion will have a typical parallel diffusion length (or mean free path) of $\ell_{\|} \sim$ $(1-2) \mathrm{R}_{\mathrm{E}}$, a rather short distance, orders of magnitude shorter than the collisional mean free path of an ion. Hence, the diffusion estimate suggests that strong wave-particle interactions in the shock transition are responsible for the scattering and acceleration of the diffuse particle component. The corresponding upstream-ion collision frequency $v_{c, u i} \simeq v / \ell_{\|}$for the $20 \mathrm{keV}$-upstream ions in the solar wind yields $v_{c, u i} \sim 0.2 \mathrm{~Hz}$, being comparable to the ion cyclotron frequency $\omega_{c i} / 2 \pi=(0.1-0.3) \mathrm{Hz}$ in the $B \simeq 8 \mathrm{nT}$ upstream to $B \simeq 30 \mathrm{nT}$ shock ramp magnetic field in the observation. The energy dependence of the diffusive acceleration thus rules out pitch-angle scattering as the responsible mechanism.

\subsection{Quasi-parallel shock reformation}

In quasi-parallel supercritical shocks there is not such a stringent distinction between the region upstream of the shock and the shock itself, as in quasi-perpendicular shocks. The foreshock, which we have discussed in some detail in the previous section, and the shock itself cannot be considered separately. This is due to the presence of the reflected and diffuse particle components in the foreshock. These, as we have seen, are the source of a large number of waves. The interaction of these waves with the shock is one of the main issues in quasi-parallel shock physics.

\subsubsection{The role of upstream pulsations}

Upstream waves excited by the diffuse ion component are shown in Fig. 33. The shock radiates energy away towards upstream in a strongly nonlinear interaction process that is modified by the steep ion-density gradient. The k-vector turns away from the magnetic field having comparable components parallel to $\mathbf{B}$ and parallel to the 

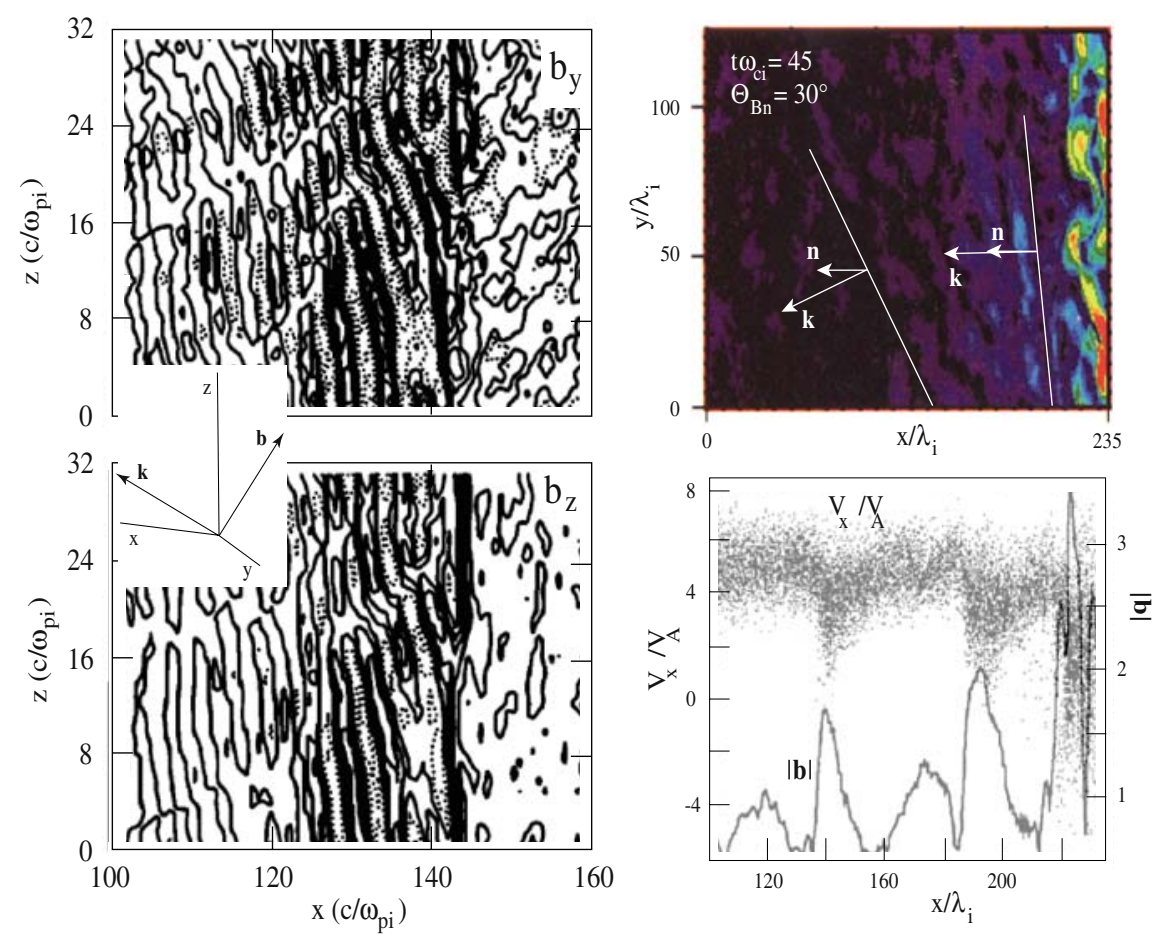

Fig. 33 Two-dimensional hybrid simulations of the evolution of upstream waves. Left column: Upstream wave in a $\mathscr{M}_{A}=2.2$ quasi-parallel shock. The shock is in the $(y, z)$-plane. Contour plot of the two (normalised to upstream magnetic field) components of magnetic fluctuations shown at $\omega_{c i} t=68$. Plane magnetic wave fronts inclined against the shock in direction $x$ of wavelengths $\sim 10 \lambda_{i}$ in $z$ and shorter in $x$. Near the shock the fronts turn parallel to the shock producing a non-coplanar magnetic component $\left|b_{y}\right|$ of same order as $\left|b_{z}\right|$. The shock is not stable, exhibiting structure in $z$ produced by the reflected upstream particles and waves. Right column: Evolution of giant magnetic pulsations (SLAMS) (after Dubouloz and Scholer 1995, courtesy American Geophysical Union). Top: The magnetic fluctuation field $|\mathbf{b}|$ at time $t \omega_{c i}=45$ and shock normal angle $\theta_{B n}=30^{\circ}$ at $\mathscr{M}_{A}=5$. Accumulation of the growing wave fronts at shock transition, their increasing amplitudes, and their turning towards becoming parallel to the shock become visible from the rotation of the two wave fronts and their $\mathbf{k}$ vectors (white). Away from the shock transition the angle between $\mathbf{k}$ and the shock normal $\mathbf{n}$ is large. Close to the shock the two vectors are about parallel. The magnetic field is in the wave front, so $\theta_{B n}$ is close to $90^{\circ}$ here. Bottom: Pulsation amplitude and ion phase space. The fluctuations evolve into large amplitude pulsations when approaching (and making up) the shock. The strong retardation of the upstream flow by the pulsations is visible in the shock-normal velocity component

shock normal n. Close to the shock, where the backstreaming ion density is high, the waves have short wavelengths, and $\mathbf{k}$ is almost parallel to $\mathbf{n}$. In the high Mach number simulations, reflected ions were artificially injected with same Mach number as the incoming flow but with much higher temperature $v_{i}=14.1 V_{A}$, forming a spatially uniform ion component. The intention was to investigate the effect of the hot reflected ions. This is shown on the right in the above figure. The result resembles the former one where a shock was generated by reflection at a wall, but the effect in the injected beam case is stronger because of the higher Mach number, proving that it is the hot diffuse ion component that is responsible for the wave and shock dynamics. The 
upstream waves have two components, $b_{z}, b_{y}$, are of low amplitude at large distance from the shock but reach very large amplitudes simultaneously in both components during shock approach while, at the same time, bending and assuming structure in $z$-direction that is different from the regular elongated shape at large distance. This deformation of the wave front implies that the shock has structure on the surface in both directions $x$ and $z$ and is no longer planar. The shock becomes locally curved on the scale of the shock-tangential wavelength. The waves deform the shock and are of same amplitude as the shock ramp. They become indistinguishable from the shock.

- Seen from the obstacle, the shock is, so to say, the first and largest-amplitude upstream magnetic pulsation.

The quasi-parallel shock-magnetic field is not coplanar, because the waves have contributed a substantial component $b_{y}$ that points out of the coplanarity plane.

Quasi-parallel shock reformation and much of its physics is predominantly due to the presence of the large-amplitude and spatially distinct upstream waves. Among these, the large-amplitude upstream pulsations are the physical generators of the shock. In addition, due to their presence, we will show below that the shock changes its local character. The shock becomes highly variable in time and position along the shock surface and is - on a smaller scale close to the shock transition- 'less quasi-parallel' (or 'more perpendicular', i.e. the shock-normal angle $\theta_{B n}$ has increased on the scale of the upstream waves). The latter is due to the out-of coplanarity-plane component that is introduced by the upstream waves. In spite of concluding this from the hybrid simulations referred to in Fig. 33, this conclusion also remains valid in full particle simulations.

The gradual evolution of the shock normal angle $\theta_{B n}$ from the quasi-parallel towards the quasi-perpendicular inclination of the magnetic field has been demonstrated by Dubouloz and Scholer (1995). These authors investigated the evolution of the shock normal angle in dependence on the distance from the shock. For two-dimensional hybrid simulations with initial shock-normal angles $\theta_{B n 0}=2^{\circ}$ and $\theta_{B n 0}=20^{\circ}$, their results are shown in Fig. 34. In both cases $\theta_{B n}$ evolves from quasi-parallel at large distance from the shock towards assuming quasi-perpendicular angles near the shock.

Qualitatively there is little difference between the two cases. When approaching close to the shock ramp, $\theta_{B n}$ is found deep in the domain of quasi-perpendicular shocks. Due to the presence of large-amplitude foreshock waves, the evolution of $\theta_{B n}$ is not smooth. Transition to quasi-perpendicular occurs for the initially nearly parallel case right at the nominal shock ramp, while for the initially more inclined quasi-parallel case it occurs at an upstream distance of about $\lesssim 100 \lambda_{i}$ from the shock. This is sufficiently far away for the upstream flow ions to feel the change in the shock normal. This transition, being on the ion scale, implies that in the region close to the shock the upstream-flow ions occasionally experience the shock as being quasi-perpendicular and become reflected.

It is most interesting that measurements of the electron distribution function by Feldman et al. (1983) at the shock do not show any noticeable differences in the electrons between quasi-perpendicular and quasi-parallel shocks. This suggests that, close to the shock transition, quasi-parallel shocks behave like quasi-perpendicular shocks as well on the electron scale. 


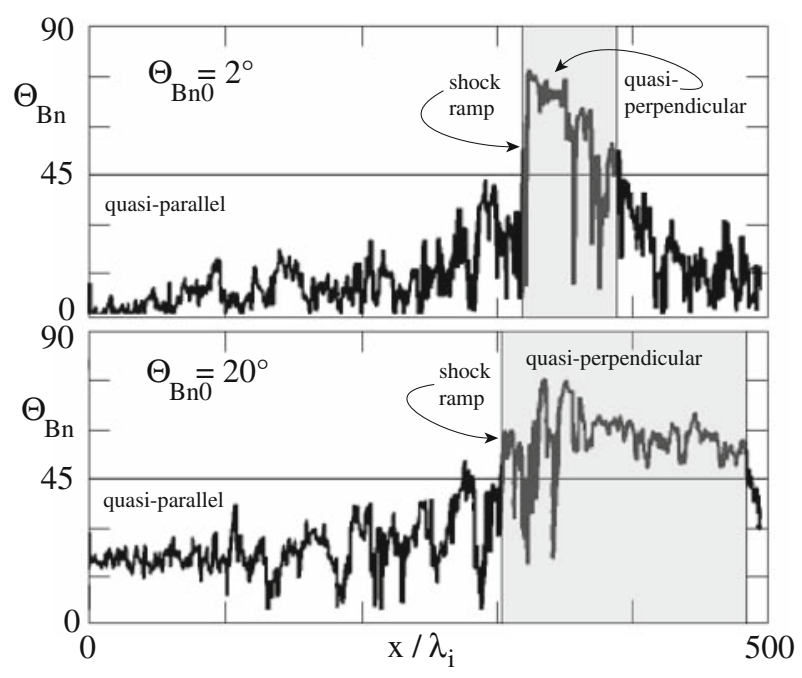

Fig. 34 The evolution of the shock normal angle $\theta_{B n}$ on distance from the shock in two-dimensional hybrid simulations for two initial quasi-parallel shock-normal angles $\theta_{B n 0}=2^{\circ}$ and $\theta_{B n 0}=20^{\circ}$, respectively (simulation results taken from Scholer 1993, courtesy American Geophysical Union). The horizontal line at $45^{\circ}$ is the division between quasi-perpendicular and quasi-parallel shock normal angles. In both cases $\theta_{B n}$ evolves from quasi-parallel direction into quasi-perpendicular direction. The shaded areas identify the quasi-perpendicular domains

The behaviour of the shock normal angle gives a rather clear identification of the location of the shock transition in the quasi-parallel case, as indicated in Fig. 34 by shading. Three distinctions can be noticed:

- at larger initial shock-normal angles the transition to quasi-perpendicular angles occurs earlier, i.e. farther upstream than for nearly parallel shocks. This is due to the strong effect of the large amplitude upstream waves;

- at larger initial shock normal angles the quasi-perpendicular shock transition is considerably broader than for nearly parallel shocks, i.e. it extends farther downstream before the main quasi-parallel direction of the magnetic field in the downstream region takes over again and dominates the direction of the magnetic field:

- at an initial shock-normal angle of $20^{\circ}$, this region is roughly $\sim 150 \lambda_{i}$ wide, implying that the magnetic field direction behind a quasi-parallel shock remains to be quasi-perpendicular over quite a long downstream distance measured from the shock ramp. For the nearly parallel shock this volume is only about $\sim 50 \lambda_{i}$ wide.

These findings have interesting implications for the downstream physics of quasiparallel shocks. For instance, applied to Earth's bow shock, where $\lambda_{i} \sim 10^{3} \mathrm{~km}$, both distances correspond to regions wider than the order of $>5 \mathrm{R}_{\mathrm{E}}$. This distance is larger than the nominal width of the magnetosheath! Thus, behind the bow shock, a substantial part of the magnetosheath plasma should behave as if the bow shock would have been a completely quasi-perpendicular shock.

Figure 35 shows a large section of the upstream foreshock region of a quasi-parallel shock. Large amplitude pulsations are formed as the result of growing ultra-low- 


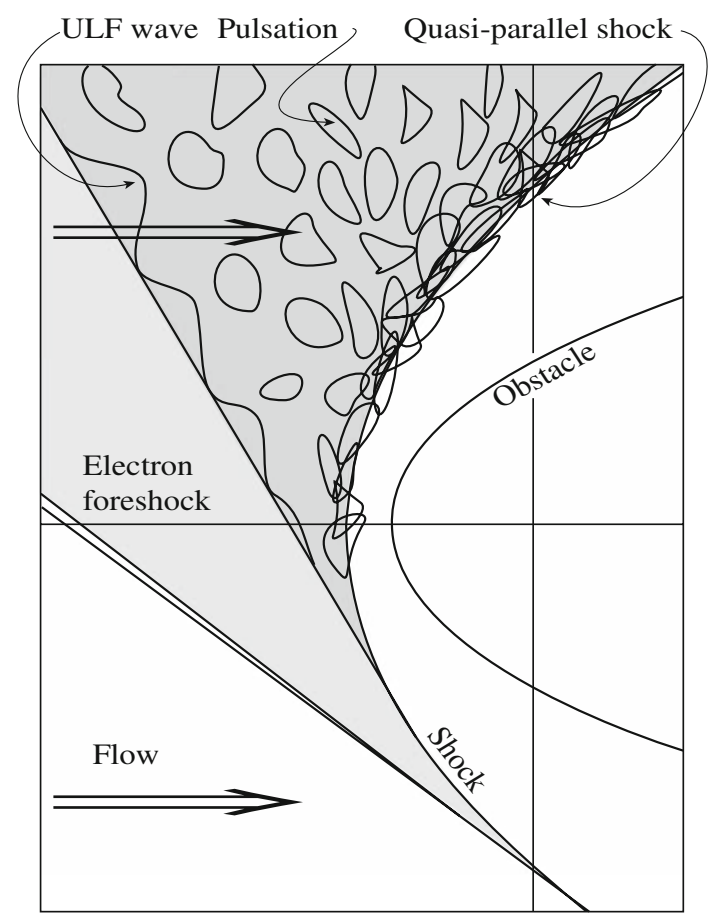

Fig. 35 Quasi-parallel supercritical shocks reform by accumulation of upstream magnetic pulsations (SLAMS) convected into the shock from the ion foreshock, thereby causing an irregular shock structure and contributing to the turning of the magnetic field into a direction to the shock normal that is more perpendicular, i.e. the magnetic field is more parallel to the shock surface with the shock surface itself becoming very irregular. It is shown schematically that the pulsations grow out of the small amplitude upstream waves which are generated in the broad ULF-wave-unstable region in greater proximity to the ion-foreshock boundary. When the ULF waves evolve to large amplitude and form localised structures and pulsation these are convected toward the shock, grow, steepen, overlap, accumulate and lead to the build up of the irregular quasi-parallel shock structure which overlaps into the downstream direction. Downstream turbulence behind quasi-parallel supercritical shocks is thus large due to pulsations that emerge from the shock and move downstream

frequency waves that are generated in the foreshock relatively close to the ion-foreshock boundary (Kis et al. 2004). These waves grow spatially until evolving into pulsations which the flow carries towards the shock. Growth, slowing down, and accumulation of the pulsations then lead to the pile-up of pulsations at the location of the quasi-parallel shock. This accumulation of pulsations at the shock position causes a quasi-parallel shock to exhibit a turbulent shock structure. Of the magnetic field, in this figure only the shock-tangential upstream field line is shown. The pulsations superpose on the background magnetic field and cause a bending of the field lines with the field turning ever more perpendicular the closer to the shock the pulsation is found. This bending is what can be called a quasi-parallel shock turning quasi-perpendicular close to the shock transition. Observations and simulations do indeed confirm the turning of the field as result of the presence of the large-amplitude magnetic pulsations.

Three representative examples of such upstream waves (Lucek et al. 2002) that support the above theoretical inference, have been plotted in Fig. 36. The shock region 


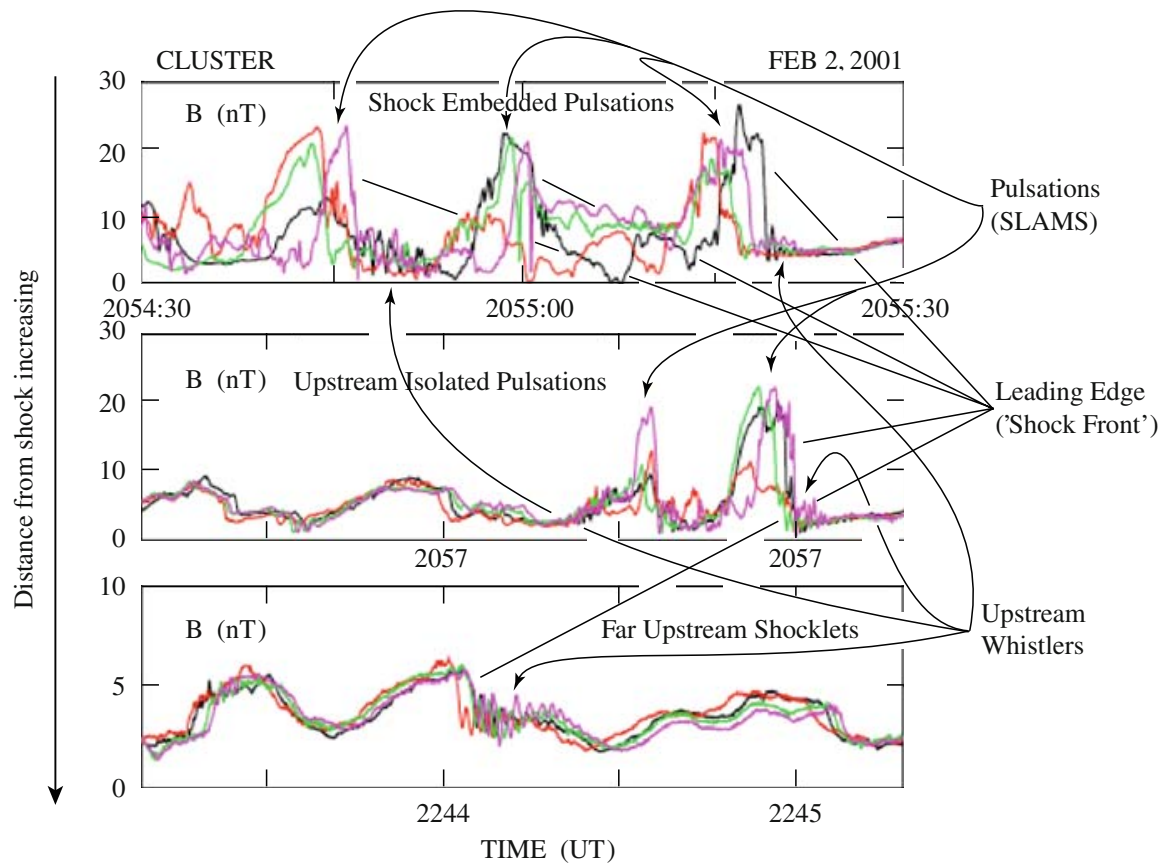

Fig. 36 Magnetic field of pulsations (SLAMS) in the quasi-parallel foreshock (data from Lucek et al. 2002) for the 4 CLUSTER (colour coded) spacecraft. Top: Accumulated pulsations in the shock transition being of small scale and large amplitude. Middle: Isolated pulsation at larger distance seen almost simultaneously at the 4 spacecraft being of larger size but lower amplitude. Bottom: Far upstream shocklet and ultra-low-frequency waves with attached upstream whistlers

consist of many embedded magnetic pulsations (SLAMS) of very large amplitudes, having steep flanks and irregular shapes. They exhibit higher frequency oscillations in the whistler mode sitting on their feet or shoulders. Pulsations in the shock are shortscale; the 4 different CLUSTER spacecraft—at spacecraft separation $<1,000 \mathrm{~km}$ - do not observe a coherent picture of a particular pulsation. The magnetic field directions differ from spacecraft to spacecraft and from pulsation to pulsation, and even for one pulsation at its front and trailing edges and the magnetic normals across a pulsation change on short spatial scales.

Full particle PIC simulations of quasi-parallel shock reformation shown in Fig. 37 strongly support this picture. On the left the reformation of the shock due to exchange of pulsations is shown. On the right this process is followed in time. The nominal shock front progresses stepwise in the upstream direction when an old magnetic pulsation is replaced by one that is newly arriving. In the phase space, the magnetic oscillations are well correlated with ion holes with the field maxima coinciding with the vertexes and the minima corresponding to the centres of the holes, i.e. to the locally largest spread in the ion distribution or, in other words, the locally highest "ion temperatures". The evolution of the corresponding velocity distributions for ions and electrons in this reformation process is given in Fig. 38. Heating of ions and electrons takes place in different phases, and there is indication of acceleration of an electron beam. 

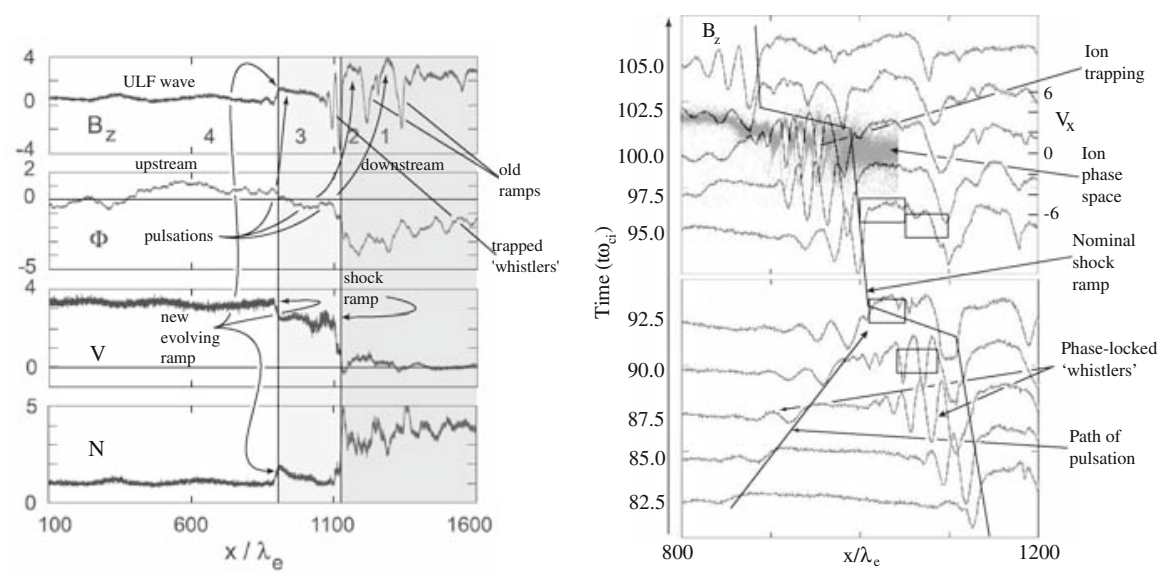

Fig. 37 1D full particle PIC simulations of quasi-parallel shock evolution (simulation data from Scholer et al. 2003, courtesy American Geophysical Union). Left from top: magnetic field $B_{z}$, electric potential $\Phi$, flow velocity $V$, density $N$ (in simulation units). Numbers indicate 3 pulsations (SLAMS). Pulsation 1 was the old shock. Pulsation 2 is the actual shock (coinciding with drop in $V$ to zero and steep increase in density and potential). Pulsation 3 is just evolving. Number 4 will become a pulsation. The actual shock ramp has some trapped whistlers. Right: $B_{z}$ (in simulation frame) for subsequent simulation times shifted upward by $\Delta t \omega_{c i}=2.5$. (The shock moves to the left into the upstream direction.) Reformation results from exchange with incoming pulsation (SLAMS). The magnetic field trace at time $t \omega_{c i}=100$ has been overlaid on the ion phase space at this time. Heavy steps show location of nominal shock ramp moving upstream until new pulsation arrives and it suddenly jumps forward by roughly $100 \lambda_{e}$. A pulsation arrives at the shock at $t \omega_{c i}=92.5$ to take over the role of the shock. In the minima of the 'whistler' field fluctuations $\left(t \omega_{c i}=100\right)$ ions are trapped, forming vortices in phase space centred around local minima of the electric potential $\Phi$ (not shown). The little boxes indicate where particle (ion or electron) phase space distributions have been determined
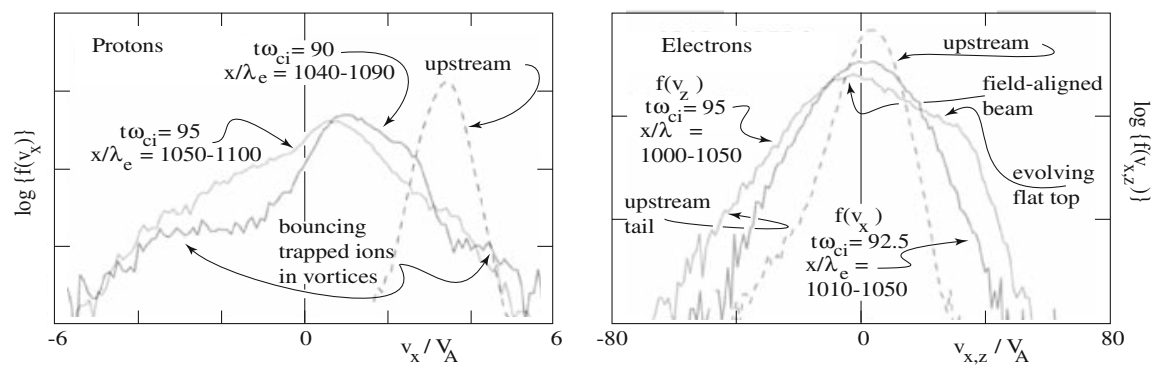

Fig. 38 Velocity distributions for different phases of shock evolution taken in the boxes of Fig. 37 (simulation data taken from Scholer et al. 2003, courtesy American Geophysical Union). Left: Proton distributions at $t \omega_{c i}=90, t \omega_{c i}=95$. The first period is in the arriving pulsation. The upstream distribution is heated with upstream tail due to trapped ions. At $t \omega_{c i}=95$ the trapped hot ion distribution damps the waves. The incident plasma is slowed down. Right: Electron distributions $f_{e}\left(v_{x}\right)$ at $t \omega_{c i}=92.5$ and $f_{e}\left(v_{z}\right)$ at $t \omega_{c i}=95$ in the new shock built up from a fresh pulsation and in the well-developed shock but along the main magnetic field, respectively. The parallel distribution is non-symmetric, heated, has an upstream tail, formation of a flat top and a remaining upstream-beam-like part similar to measured (Feldman et al. 1983) 


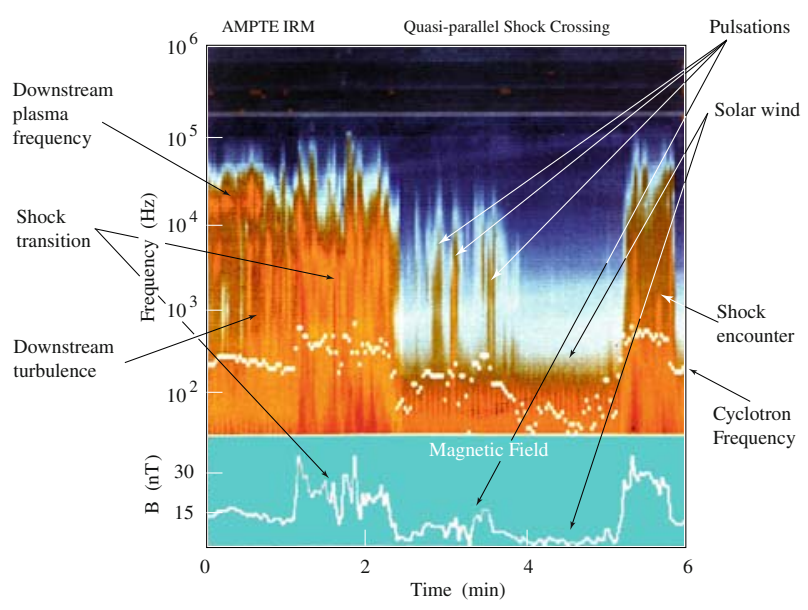

Fig. 39 An in situ quasi-parallel shock crossing in the plasma wave spectrum. The shock appears as broadband emission. The magnetic field variation in the lower part exhibits the presence of large amplitude pulsations SLAMS. In plasma waves these appear as short broadband signals on the background of moderate foreshock wave activity. Pulsations are thus very active in exciting plasma waves, resembling shock elements (from Treumann and LaBelle 1986, unpublished)

\subsection{High-frequency waves and radiation}

Quasi-parallel shocks form and reform in the course of an interplay between the shock-produced upstream diffuse energetic-ion component and the magnetic pulsations which grow out of the wave-excited upstream waves, and are amplified in the interaction with the diffuse ions during their approach of the shock. Quasiparallel supercritical (high Mach number) shocks do not form in the absence of diffuse upstream ions and the waves that are excited by them.

This important conclusion raises the question for the role of high-frequency plasma waves, since these are the waves which participate in the generation of radiation. Figure 39 shows that the plasma-wave signature of a quasi-parallel shock is a very broadband turbulent emission, and that the upstream pulsations show the same signatures as the shock. Another example of waves below $80 \mathrm{kHz}$ is shown in Fig. 40 obtained with the highly sensitive CLUSTER-quartet wave-receiver. Again, the shocks appear as very broadband signals, with the plasma frequency mapping the density. In addition one finds the indication of harmonic emission at twice the plasma frequency.

Shocks are frequently referred to as sources of radiation. Famous examples are supernova shocks, which are visible in almost all wavelengths, from radio through visible light up to x-rays (e.g., Dickel and Wang 2004). Supernova shocks are relativistic shocks which are not treated here. ${ }^{20}$ Other examples are solar type II shocks; these are non-relativistic shocks. Their main radiation signatures are in radio waves.

\footnotetext{
20 Their Mach numbers range from weakly relativistic to highly relativistic, but the energy per particle in them remains to less than the rest energy of an electron, $\lesssim m_{e} c^{2}=0.511 \mathrm{MeV}$, which allows us to treat them classically. This does not hold anymore for the central supernova engine which drives the flow and which in some cases results in the generation of ultra-relativistic jets. There the shocks become
} 


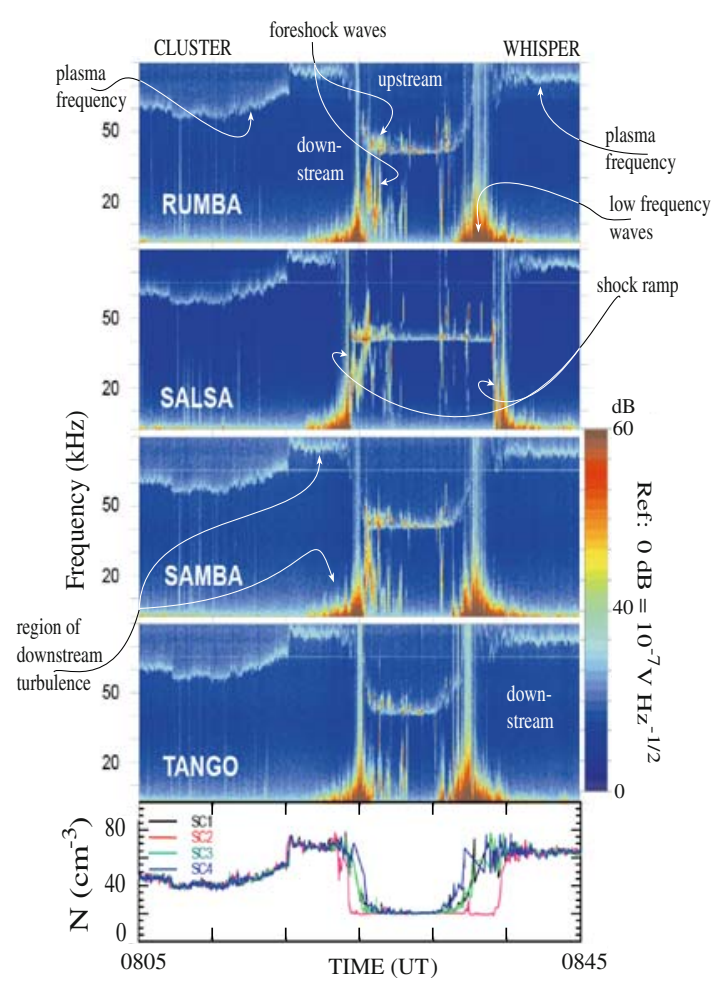

Fig. 40 A CLUSTER spacecraft passage across Earth's bow shock on December 22, 2000. The spacecraft cross the downstream magnetosheath region (left) and the shock, enter the upstream solar wind region and back downstream. The figure shows broadband $(\sim 0-80 \mathrm{kHz})$ plasma wave electric field spectra from all four CLUSTER spacecraft (with their somewhat childish names Rumba, Salsa, Samba, Tango). The uppermost light blue emissions are at the electron plasma frequency $f_{p e}=\omega_{p e} / 2 \pi$ which maps the local plasma density $N$ estimates of which are shown in the lowest panel. Passages of the shock are signalled by intense broadband waves starting at low frequencies and being correlated with steep drops in $N$. In the low density upstream solar wind the spots of strong intensification of $f_{p e}$ indicate contact with the electron foreshock boundary beam and excitation of Langmuir waves. These are highly structured, sometimes stretching above, sometimes hanging down below $f_{p e}$. Near the shock intermediate frequency emissions are also seen (data taken from Décréau et al. 2001)

The radiation that is occasionally emitted from nonrelativistic shocks is restricted to the radio wave range. Nonrelativistic shocks do not generate X-rays or visible emissions because, first, of their comparably low energy per particle, which is less than the rest energy of an electron $\ll m_{e} c^{2}$, second, because of their low energy transmission rate (particles are not retarded from flow speed to rest) and, third, because of the low 'emission measure'. ${ }^{21}$ Moreover, since magnetic fields are weak and the

\section{Footnote 20 continued}

non-classical, in this case not only radiation losses but also particle generation must be taken into account in their description.

21 The emission measure is defined as $E M=\int_{\operatorname{los}} \mathrm{d} s \Delta N_{e}^{2}(s)$, with $\Delta N_{e}$ the number density of radiating particles. In other words, the square of the radiating particle number in the volume that contributes to the emission of x-rays, integrated along the line-of-sight $s$, is low. 


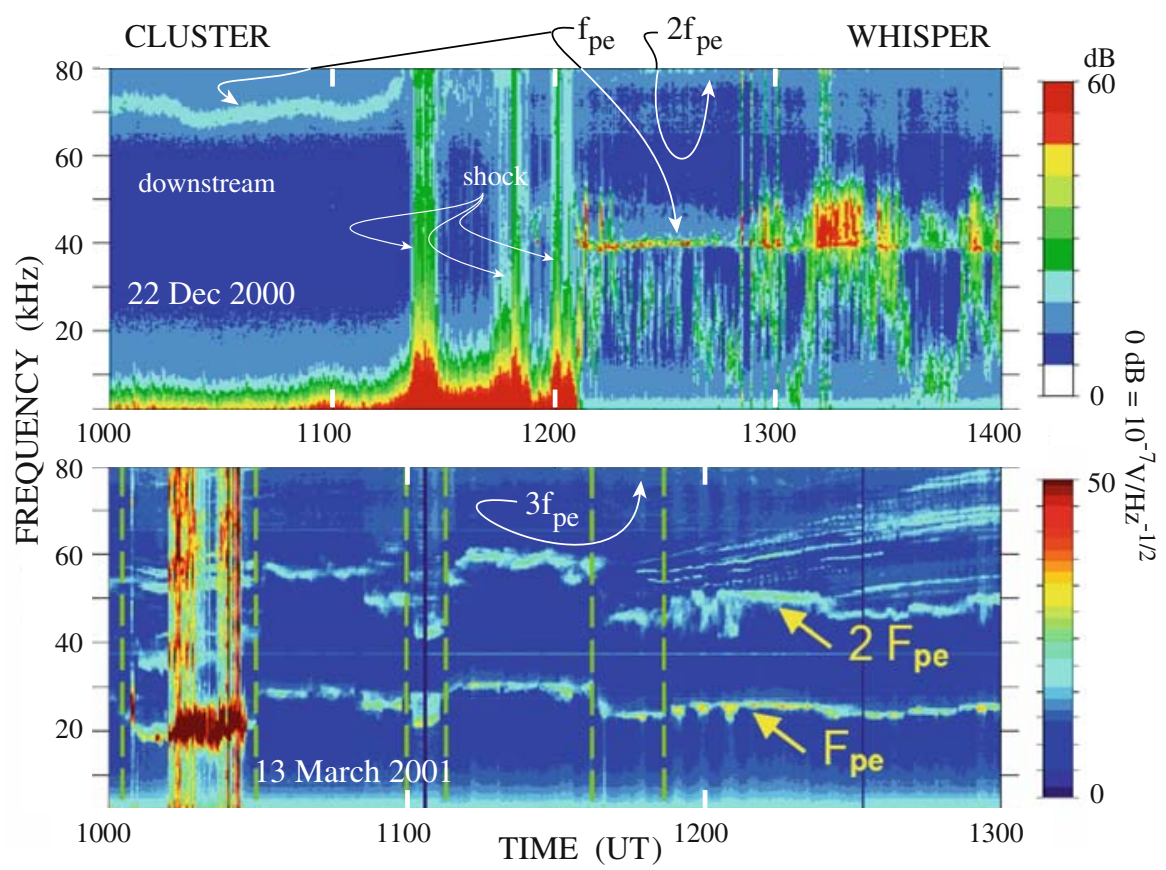

Fig. 41 Observations of electromagnetic radiation from the electron foreshock, following the multiple CLUSTER shock crossings on two different days. Top: Typical $f_{p e}$ and lower frequency emissions from 1,210 to $1,250 \mathrm{UT}$ at the electron foreshock boundary and $2 f_{p e}$ radiation near $80 \mathrm{kHz}$. Weak emission just above $f_{p e}$ is seen with upper cut-off decreasing in frequency. At later times deeper in the shock broadband electrostatic emissions at $f_{p e}$ occur. These correlate with broadening of the harmonic radiation to lower frequencies. Bottom: Three hours of low upstream density when the spacecraft was close to the foreshock boundary. $f_{p e}$ is accompanied by harmonic emission not much less intense than $f_{p e}$. During broadening of $f_{p e}$ the harmonic emission broadens downward. Near $80 \mathrm{kHz}$ a faint third harmonic band $\sim 3 f_{\text {pe }}$ can be identified. The band splitting in the harmonic radiation between 1,000 and 1,010 UT is of interest. Many narrow-band upward drifting equally spaced in frequency radiation bands after 1,140 UT can be identified. They have no counterpart in $f_{p e}$ and may come from a remote source related to sharp changes in density (drop in $f_{p e}$ ) at 1,140 UT. The dashed vertical lines mark changes in the magnetic field (not shown here), when its direction abruptly turned (after Trotignon et al. 2001, courtesy EGU, and Trotignon, private communication)

ratio of electron plasma to electron cyclotron frequency, $\omega_{p e} / \omega_{c e}>1$, is larger than one, gyro-synchrotron emission is unimportant. Thus, presumably the only means of how free-space radiation of frequency $\omega \gtrsim \omega_{p e}$ can be produced is via plasma wave emission.

In the heliosphere, radio emission from collisionless shocks is a widely studied field that includes type II solar radio bursts, interplanetary type II bursts, CMEdriven radio bursts and radio emissions from planetary bow shocks. While solar bursts can be observed from Earth, ${ }^{22}$ most of the other emissions have been discovered only from aboard spacecraft. Figure 41 shows two cases of CLUSTER observations

\footnotetext{
22 Because of the high electron density in the solar corona, their emission frequency $(f \gtrsim 100 \mathrm{MHz})$ is higher than the ionospheric reflection frequency $\left(f_{\text {ion }} \sim 10 \mathrm{MHz}\right)$.
} 
when the spacecraft crossed the bow shock and moved into the electron foreshock (Trotignon et al. 2001). Here the upstream density was high with plasma frequency near $40 \mathrm{kHz}$. The intense spots in $f_{p e}$ between 1,200 and 1,300 UT indicate that the spacecraft is in contact with the electron foreshock-boundary field line. Broadband wave modes around $\sim \frac{1}{2} f_{p e}$ indicate beam modes, though no frequency drift is detectable in the bursts. However, during this time interval the density is low enough for a faint free-space mode emission to occur at about $\sim 80 \mathrm{kHz}$. This frequency is just about two times the local $f_{p e}$, witnessing local generation of radio emission from the foreshock boundary. At later times when the spacecraft moves deeper into the foreshock -indicated by the widening of the plasma wave spectrum to both sides, up and down from $f_{p e}$ - the radio emission becomes diffuse and broadband shifting to frequencies lower than the second harmonic of the plasma frequency. Here, lower frequency modes close to $f_{p e}$ actively participate in the radiation which, however, could also arrive from the shock ramp, if it would have been generated there.

The lower panel in Fig. 41 is at much lower upstream density, showing the typical case of Langmuir waves generated near $f_{p e}$ and radio emission at almost precisely $2 f_{p e}$. Near $80 \mathrm{kHz}$ a faint indication of the presence of an emission at the third harmonic $\sim 3 f_{p e}$ can be recognised. The dashed vertical lines in this panel indicate those times when the magnetic field direction changed abruptly with the changes in magnetic field magnitude and the changes in density remained comparably small. The emissions in the plasma frequency and its harmonic radiation are well correlated during the entire event. Obviously CLUSTER was constantly close to the foreshock boundary as only intensification in $f_{p e}$ is seen but neither 'hair' nor 'beards' evolve except during a period shortly after 1,200 UT in the three yellow spots in $f_{p e}$, when the harmonic emission extends to lower frequencies together with the development of a 'beard'.

At an early time just before 1,020 UT, the harmonic emission splits into two narrow bands, which is an effect that had first been discovered long ago in solar type II bursts. ${ }^{23}$ Suddenly after 1,200 UT (following the drop in plasma density at 1,140 UT) drifting emissions with increasing frequencies evolve. Both features, the splitting of $2 f_{p e}$ and these drifting bursts are not understood yet. The latter might be related to the abrupt changes in density and plasma frequency in this panel. These density changes are accompanied by changes in the magnetic field. The banded drifting emissions cannot come from a remote source because the low frequencies arrive first. They come from a nearby source, most probably from the shock.

\subsubsection{Theory}

Theory of shock-emitted radiation is based on plasma processes. Under the prevailing collisionless conditions in the shock and foreshock plasmas, these processes refer to wave-wave coupling as the main generation mechanism. Direct emission from particles is unimportant, because the energy losses a particle experiences during retarding

\footnotetext{
23 This kind of band-splitting is typical for solar type II bursts. Surprisingly, its mechanism has not yet been understood so far (for a contemporary account of the proposed mechanism the reader is referred to the Chapter on solar coronal shocks in Balogh et al. (2009)).
} 
at or reflection from a shock are not transformed into radiation that would be intense enough for observation.

Expecting that - presumably — direct particle involvement in radiation is improbable (maybe with two exemptions ${ }^{24}$ ) we are left with a small number of possible mechanisms, which belong to the class of wave-wave interactions in plasma turbulence. In a perturbative approach, the most probable of these are three-wave processes, 'collisions' between three 'quasi-particles'. This kind of 'elastic' interaction conserves energy and momentum and can, symbolically, be written as

$$
\mathrm{L}+\mathrm{L}^{\prime} \rightarrow \mathrm{T}, \quad \mathrm{L} \equiv\left\{\omega_{\mathrm{L}}\left(\mathbf{k}_{\mathrm{L}}\right), \mathbf{k}_{\mathrm{L}}\right\}, \quad \mathrm{L}^{\prime} \equiv\left\{\omega_{\mathrm{L}}^{\prime}\left(\mathbf{k}_{\mathrm{L}}^{\prime}\right), \mathbf{k}_{\mathrm{L}}^{\prime}\right\}, \mathrm{T} \equiv\{\omega(\mathbf{k}), \mathbf{k}\}
$$

Here $L$ stands for longitudinal, and $T$ for transverse. The emitted radiation of frequency $\omega$ and wave number $\mathbf{k}$ propagates in the free-space mode and is thus a transverse electromagnetic wave. Its two intense 'mother waves' are assumed to be longitudinal (i.e. electrostatic) waves of high frequency and thus large energy $\hbar \omega_{\mathrm{L}}$ per plasmon. The frequencies $\omega_{\mathrm{L}}\left(\mathbf{k}_{\mathrm{L}}\right)$ etc. depend on wave number through the electrostatic dispersion relation $D_{\mathrm{L}}\left(\omega_{\mathrm{L}}, \mathbf{k}_{\mathrm{L}}\right)=0$ of plasma waves. Normally, this dispersion relation may become quite complicated.

There are two free space modes, the ordinary and the extraordinary mode which, in order to leave the plasma and escape as radiation, must exceed some lower cut-off frequency. For the ordinary mode

$$
\omega^{2}=\omega_{p e}^{2}+k^{2} c^{2}
$$

this cut-off is the plasma frequency at very long wavelengths $k=0$. Since the speed of light $c \gg v_{e}$ is large, the radiated wavelength is much longer than any of the other wavelengths involved. This is immediately recognised when comparing the above ordinary wave dispersion relation with the Langmuir wave relation, with $L \equiv \ell$,

$$
\omega_{\ell}^{2}=\omega_{p e}^{2}+3 k_{\ell}^{2} v_{e}^{2}
$$

which has the same structure. As long as the electron thermal speed $v_{e} \ll c / \sqrt{3}$, one has $k_{\ell} \gg k$, and the wave number of the radiated mode is practically zero. Momentum conservation

$$
\hbar \mathbf{k}_{\mathrm{L}}+\hbar \mathbf{k}_{\mathrm{L}}^{\prime}=\hbar \mathbf{k} \approx 0
$$

of the three interacting quasi-particles (longitudinal plasma waves or plasmons) becomes $\mathbf{k}_{\mathrm{L}} \approx-\mathbf{k}_{\mathrm{L}}^{\prime}$, implying that the interaction selects counter-streaming electrostatic waves. Any process that is capable of generating counterstreaming Langmuir waves, for instance, or other longitudinal waves that satisfy this condition, can contribute to the generation of radiation. From energy conservation

$$
\hbar \omega_{\mathrm{L}}+\hbar \omega_{\mathrm{L}}^{\prime}=\hbar \omega
$$

$\overline{24}$ See below at the end of this section. 
of the three 'quasi-particles' involved, one finds for merging counter-streaming Langmuir waves that $\omega \approx 2 \omega_{p e}$. This is the origin of and the simplest mechanism for the generation of the celebrated $f \approx 2 f_{p e}$-second plasma-harmonic radiation and was proposed more than half a century ago by Ginzburg and Zheleznyakov (1958) as an explanation for the observation of solar type II and type III radio bursts.

In this simple reasoning we have completely neglected not only the contribution of the Langmuir wave number (which turns out not to be important in magnitude, it just shifts the emitted frequency a tiny amount up in frequency) but also the fact that the electric field of Langmuir waves is polarised along $\mathbf{k}_{\ell}= \pm k_{\ell} \mathbf{B} / B$, while the electric field of the transverse emitted electromagnetic radiation must necessarily be polarised perpendicular to $\mathbf{k}$. Since the electric field after collision and annihilation of the two Langmuir waves involved remains oscillating along the magnetic field, the emission is preferably directed perpendicular to the magnetic field. It is easier to radiate in the extraordinary than in the ordinary free space mode. The extraordinary mode has a more complicated dispersion relation; in a dense plasma with $\omega_{p e} \gg \omega_{c e}$ it has a slightly higher cut-off frequency. But the argument about the smallness of $k \ll k_{\ell}$ holds also in this case. Radiation at the second harmonic $\omega \approx 2 \omega_{p e}$ should therefore be polarised preferentially perpendicular to the magnetic field in the extraordinary mode.

Foreshock emission has not been found to show any preference in polarisation, and emission is not only in the second harmonic but has also been detected close to $\gtrsim \omega_{p e}$ and at the third harmonic $\sim 3 \omega_{p e}$. Such emissions require different waves to be involved for which a number of mechanisms have been proposed, of which none could be ultimately verified so far. Since radiation is energetically negligible, as we have mentioned above, the whole problem could be put aside. However, since the assumptions made in every radiation mechanism contain important information about the source region, the problem of radiation production in collisionless shocks remains to be tantalisingly urgent and awaits resolution.

At the shock, electrons are accelerated along the shock-tangential field line into gentle beams which excite forward Langmuir waves only. This requires some mechanism that backscatters a substantial percentage of waves and inverts their direction. Three processes are capable of doing this: modulation instability respectively collapse, scattering of Langmuir waves off thermal ions, and scattering of Langmuir waves off ion-sound waves, (for an early review of the latter two mechanisms cf., e.g., Tsytovich 1970).

The modulational instability generates ion-sound waves via the ponderomotive pressure force $\mathbf{F}_{\mathrm{pmf}}=-\left(e^{2} / m_{e} \omega_{p e}^{2}\right) \nabla\left|\mathbf{e}_{\ell}\right|^{2}$ of high-frequency Langmuir waves, $\mathbf{e}_{\ell}(\mathbf{r}, t)$. When becoming locally large amplitude, the waves structure the plasma into a chain of cavities in which the Langmuir waves become trapped. This process generates long wavelengths. It is described by the Zakharov equations ${ }^{25}$ for the combined evolution of the Langmuir wave field and the density variation $\delta N$, respectively,

$$
\frac{\partial^{2} \delta N}{\partial t^{2}}-c_{\mathrm{ia}}^{2} \nabla^{2} \delta N=\frac{\epsilon_{0}}{m_{i} N} \nabla^{2}|\mathbf{e}|^{2}, \quad \frac{\partial \mathbf{e}}{\partial t}+\frac{3 \omega_{p e}}{2} \lambda_{\mathbf{e}}^{2} \nabla^{2} \mathbf{e}=\frac{\omega_{p e}}{2 N} \mathbf{e} \delta N
$$

\footnotetext{
25 See any book on nonlinear plasma theory, e.g. Treumann and Baumjohann (1997).
} 
The first equation is a driven wave equation for the density variation which, for slow time variations, when the derivative with respect to time is neglected, simply implies pressure balance between the ponderomotive pressure on the right and plasma pressure on the left, i.e. proportionality $\delta N \sim-|\mathbf{e}|^{2}$. The density variation anti-correlates with the field pressure, which corresponds to local caviton formation: high wave pressure expels plasma locally. The second equation is a nonlinar Schrödinger equation for the evolution of the wave amplitude in the presence of a thermal plasma.

The Langmuir waves trapped in the cavitons bounce back and forth, which naturally creates counter-streaming waves of equal intensity with opposite wave numbers. During collapse the cavities shrink in size, the wave numbers and momenta of the waves increase, and the wave energy density increases because of the shrinking volume. This yields both the counter streaming Langmuir waves being localised in the same region and, in addition, large radio emissivity.

This process - as beautiful as it might be — has not been confirmed experimentally, neither in observations nor in full particle simulations. Observed wave intensities are too low in the electron-foreshock boundary and electron foreshock, and the density variations did not indicate the presence of the expected cavities. Simulations, on the other hand support quasilinear evolution and wave scattering off thermal ions.

We note, however, that the most recent detection of very strong electric fields in the shock ramp (Bale and Mozer 2007) might indicate that it is not the electron foreshock where one should expect caviton and collapse to work and cause the most intense radiation, rather it might be the very shock transition where shock radiation is generated by similar processes. The observed band splitting and high intensities might have been caused by Langmuir-caviton collapse in the shock ramp/transition region (Treumann and LaBelle 1992). ${ }^{26}$

Scattering of Langmuir waves off thermal ions reads symbolically $L+i \rightarrow L^{\prime}+i^{*}$, where primed quantities are after the collision, and the asterisk indicates excitation of the ion. The ion is merely excited, while the scattered Langmuir wave has changed direction and lost momentum, i.e. it has attained a longer wave number and lower frequency. The same process also applies to scattering off ion-sound waves as $L+I S \rightarrow$ $L^{\prime}$. The scattered Langmuir waves in this case change direction by absorbing the sound. Radiation at the third plasma harmonic is generated by a four-wave process. This is also favoured by caviton formation and collapse since the waves in this case are all confined to one and the same volume. However, other mechanisms (like stochastic growth in a fluctuating density background plasma) have also been proposed. These processes are of the nature of wave-wave interactions, and thus their efficiencies are perturbatively low, being proportional to the product of the involved relative wave intensities. Since the latter are usually low, the efficiencies are very small.

Generation of radiation at the fundamental $\omega \gtrsim \omega_{p e}$ in a three-wave process requires the presence of a low frequency wave. Ion-acoustic waves are one candidate, other

\footnotetext{
26 Another possibility is that kinetic processes dominate the evolution of the waves. Then BGK modes may be produced. Their involvement in the generation of radiation has not yet been investigated in detail. It is intriguing to think of the generation of so-called 'backbone radiation' from solar type II shock waves in view of generation of BGK modes. Since, as has been noted at an earlier place in this review, BGK modes accelerated electrons into fast beams, one may imagine that such beams are produced in the shock ramp/transition region where, at the high plasma densities they may generate plasma waves and radiation.
} 
candidates are lower-hybrid waves, Buneman waves, the modified-two stream instability, various kinds of drift waves, and also electron acoustic waves or electron cyclotron waves. In particular, the latter are present in the foreshock region and thus can combine with Langmuir waves to generate fundamental radiation slightly above the plasma frequency.

The two exemptions, when particles become involved, are the above mentioned scattering of Langmuir waves off ions, and the so-called electron-cyclotron maser instability (for a contemporary review see, e.g., Treumann 2006).

The electron-cyclotron maser instability operates directly on the free space mode avoiding any intermediate step like three-wave processes or particle scattering. It is thus of vital interest in generation of radiation. However, it requires a particular form of the electron distribution with a velocity space gradient in the perpendicular direction $\partial F_{e}\left(v_{\|}, v_{\perp}\right) / \partial v_{\perp}>0$, a dilute hot electron distribution, and low cold electron density. It is barely known whether such distributions can be realised at a shock. However, if they are in some place, then the cyclotron maser instability will outrun all other mechanisms and directly feed the free-space electromagnetic radiation modes.

Radiation will then be at a harmonic of the electron cyclotron frequency, which is a severe restriction if the magnetic field is low and the density high as, for instance, near the Earth's bow shock or any interplanetary shock wave. Regions of very low density and much stronger magnetic fields, than so far observed in collisionless non-relativistic shocks, are the only candidates for this radiation source. At Earth's bow shock, the ratio of electron plasma-to-cyclotron frequency is $f_{p e} / f_{c e} \sim 50$, when taking into account the compression of plasma and magnetic fields in the shock. Escaping radiation thus requires excitation of high cyclotron harmonics. Such high harmonics have very low growth rates and are thus strongly suppressed.

One may expect that the electron-cyclotron maser instability works in relativistic collisionless shocks when these amplify the shock-magnetic field via the relativistic Weibel instability. Because $f_{p e} / f_{c e} \sim \gamma_{\text {rel }}$ increases with the relativistic $\gamma$-factor, this requires large magnetic amplification factors. On the other hand, in the strong magnetic fields of compact objects, the electron-cyclotron maser instability becomes a viable radiation mechanism as well for shocks.

\subsubsection{Electric fields}

Behlke et al. (2003) report another interesting property of magnetic pulsations in the shock transition region where they overlap to form the quasi-parallel shock. Measurements of the electric cross-SLAMS potential identify a substantial unipolar drop in the electric potential of the order of several $100 \mathrm{~V}$, corresponding to a potential ramp, when passing from upstream to downstream across the pulsation. Such a drop signifies the presence of an electric field in one direction across the pulsation. Taking the mean size of a pulsation to be roughly $1,000 \mathrm{~km}$, the mean electric field is $\langle E\rangle \simeq 400 \mathrm{mV} \mathrm{m}^{-1}$. However, this field drops mainly at the leading edge of the pulsation. Such a field presumably corresponds to a steep pressure gradient in the pulsation. It could, in principle, also be generated by an anomalous collision frequency, in which case it would require generation of dissipation. This remains to be tested by further observations and data analysis. 


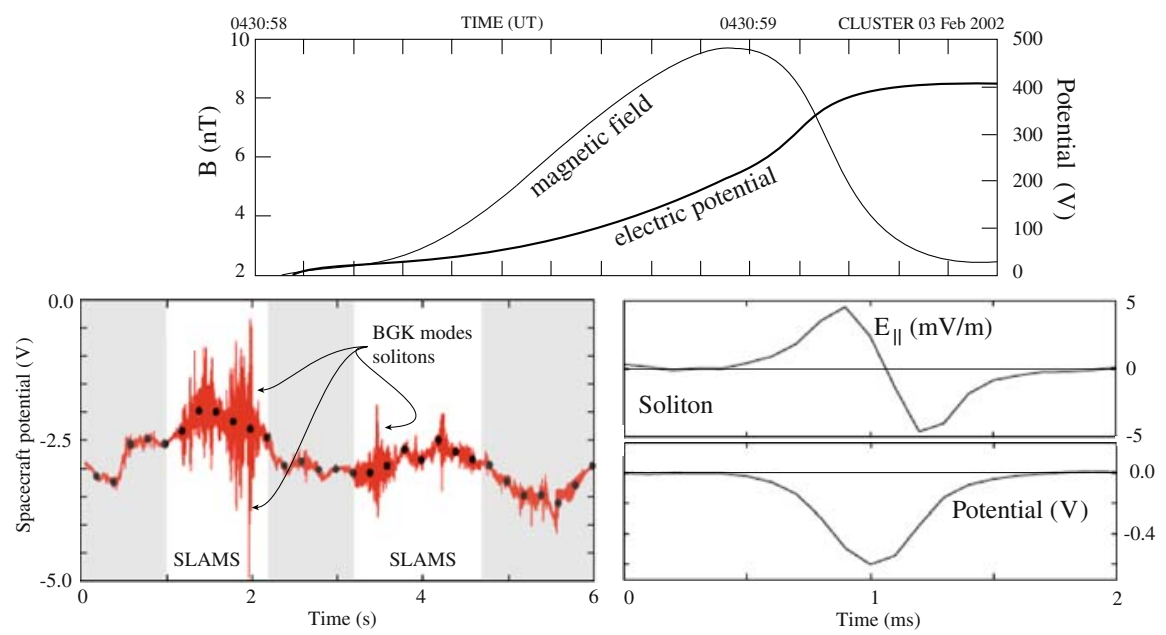

Fig. 42 Observation of electric field structures in large magnetic pulsations (SLAMS) in the quasi-parallel shock transition region (data taken from Behlke et al. 2003, courtesy American Geophysical Union). Structures on three different time scales are shown, corresponding also to three different spatial scales. Top: CLUSTER passage across on (moderately large amplitude) magnetic pulsation in the shock transition. The (smoothed) magnetic field structure is a slightly steepened magnetic bump. The stationary parallel electric potential field across this structure shows a potential ramp with steep gradient at the leading edge of the pulsation. The potential drop of $\sim 400 \mathrm{~V}$ corresponds to an electric field of $\sim 0.47, \mathrm{~V} \mathrm{~m}^{-1}$. Note that the time scale in this panel is $90 \mathrm{~s}$. Bottom left: Six seconds of a CLUSTER passage through the shock transition. The black dots show the spacecraft potential variation which maps the local density variation. Overlaid is the high frequency WHISPER trace of the plasma frequency. In the magnetic pulsation regions (white) the plasma frequency exhibits huge excursions to both sides similar to those seen in the overview Fig. 41 on wave observations. These excursions trace the BGK (nonsymmetric) modes and (symmetric) solitons. Bottom right: One example of one of the solitons on a $2 \mathrm{~ms}$ time scale. It is nicely seen how symmetric the parallel potential trough and the corresponding bipolar parallel electric field shape look like in the solitary wave structure

The measurements of Behlke et al. (2003) anticipated the more recent report of strong electric fields in the shock by Bale and Mozer (2007). In addition to this observation it was found that the single pulsations were subject to a fairly large number of high frequency/Debye scale structures in the electric field seen in the WHISPER recordings (bottom panel), which belong to electron holes (BGK modes) or solitons which form in the pulsation gradient-regions as shown in Fig. 42. The bipolar electric field and unipolar potential across one soliton is also shown there.

The occurrence of these intense nonlinear electrostatic electron plasma waves at the quasi-parallel shock transition is intriguing. It forces one to draw another very important main conclusion: that quasi-parallel shocks are sources of electron acceleration into electron beams, which are capable of moving upstream along the magnetic field and exciting electron plasma waves at intensity high enough for generating nonlinear solitons and electron holes (BGK modes). ${ }^{27}$ Presumably, this becomes possible only when the magnetic field in the supercritical quasi-parallel shock-transition region changes from quasi-parallel to quasi-perpendicular on the electron scale $\sim \lambda_{e}$.

$\overline{27}$ See also the previous footnote. 
This conclusion has important consequences for collisionless shock physics. Supercritical collisionless-nonrelativistic_-shocks are always quasi-perpendicular on the electron scale. This conjecture probably also holds for relativistic shocks though for other reasons (like the generation of transverse magnetic fields by the Weibel instability, which becomes dominant in relativistic shocks (see, e.g., Jaroschek et al. 2004)). Thus, the true quasi-parallel shock physics cannot be properly elucidated when ignoring electron effects as is done, for instance, in hybrid PIC simulations and is assumed to hold in the common application of MHD theory to astrophysical shocks.

On the other hand, since shocks are treated there as being predominantly perpendicular, MHD theory is accidentally close to reality even though the huge MHD cross-shock scales are many orders of magnitude apart from the actual scales of collisionless shock transitions. The signature of astrophysical shocks is the signature of the radiating shock-accelerated particle component which fills the extended shock environment of the foreshock and the downstream turbulent region. Thus it is related to the capability of shocks of accelerating particles, in particular electrons, high enough in particle number and energy in order to generate sufficiently intense radiation in various energy bands from radio into the UV and soft-X rays that can be observed remotely.

\section{Particle acceleration in supercritical shocks}

\subsection{Introduction}

The astrophysical interest in shock acceleration of charged particles is twofold. Firstly, shocks are believed to be involved in the generation of high energy cosmic ray particles. In the first place, this concerns the acceleration of ions and heavy nuclei. On the other hand, shock acceleration is also believed to be involved in acceleration of high energy electrons. While the former are manifest in particle radiation, generation of high-energy gamma-rays and gamma ray line emissions, the latter produce electromagnetic radiation from radio waves to the high-energy X-ray continuum via the plasma, synchrotron and bremsstrahlung radiation processes.

The physics of the processes involved in the production of cosmic rays is based mainly on the assumption of the diffusive first-order Fermi mechanism (of the many available reviews we just note Blandford and Eichler 1987; Diehl et al. 2002), schematically shown in Fig. 43. In this mechanism the shock appears as an infinitesimally thin boundary that separates two media of different flow velocities. The particles gain energy by bouncing back and forth many times across the shock between the two sides of the shock. Clearly, the interest in the very shock physics is meagre in this case. Diffusive shock acceleration, however, requires pre-acceleration of the particles to sufficiently high energy ${ }^{28}$ in order to enter into the diffuse acceleration process of the Fermi-cycle, and here the shock physics comes into the play.

\footnotetext{
28 This means pre-accelerating particles until they gain gyro-radii that are large enough for the shock to be considered as an infinitely thin boundary that separates two regions of different plasma properties.
} 


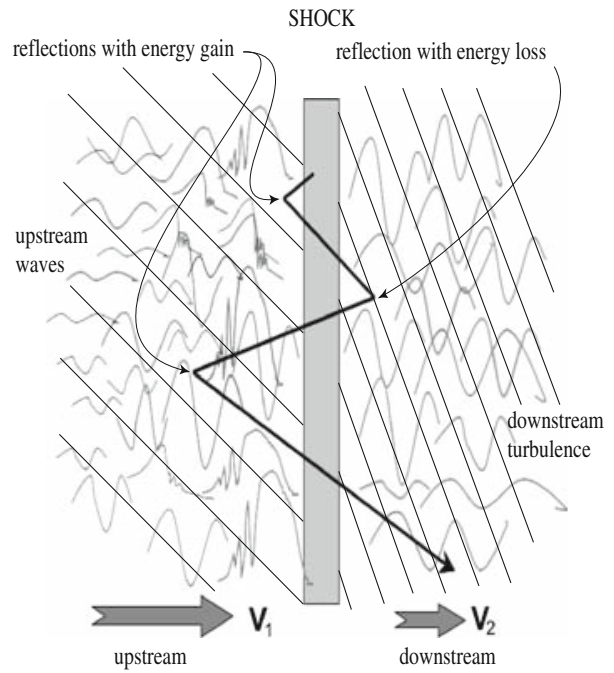

Fig. 43 Schematic of the acceleration mechanism of a charged particle in reflection at a quasi-parallel $\left(\theta_{B n}<45^{\circ}\right)$ supercritical shock. The upstream plasma flow (left, $\left.\mathbf{V}_{1} \gg \mathbf{V}_{2}\right)$ contains upstream waves: shocklets, whistlers, pulsations. The downstream plasma (right) is turbulent. An energetic particle injected from the shock to upstream is reflected in an energy gaining collision with upstream waves, moves downstream where it is reflected in an energy loosing collision back upstream. It loses energy because it overtakes the slow waves, but the energy loss is small. Returning to upstream, it is scattered a second time again, gaining energy. Its initially high energy increases successively until escape from the shock to free space as a Cosmic Ray particle. Energy gain is at the expense of the upstream flow. However, the number of energetic particles is small and the energy gain per collision is also small. Retardation of the upstream flow is much less than the retardation it experiences in the interaction with the shock-reflected low energy particles and the excitation of upstream turbulence

In many systems pre-acceleration is provided by different means than shocks with the particles that become accelerated having been produced somewhere else, and the shocks serving as the final agents of pushing their energies up into the cosmic ray range. In this case interest is mainly in the energy limit and spectrum of particles that can be obtained in shock acceleration. For the purposes of astrophysical application to many such problems, it suffices to simply assume upstream and downstream turbulent spectra and consider the particles as test particles, the interaction of which does not affect the physics of shock formation. This approach is justified as long as the number of particles is small and does not react on the shock to modify it. One may expect that a state of equilibrium is reached where the shock just accelerates as many particles as it can digest without becoming destroyed.

Such equilibria are known as cosmic ray-mediated shock states. They don't interest us here as, from the point of view of the real shock physics, two other problems are of greater importance. The first is the generation of the turbulent spectra upstream and downstream of the shock that are needed for the Fermi mechanism: the problem of selfconsistent shock turbulence. The second problem concerns the question of whether a supercritical shock by itself is capable of injecting particles out of the background flow into the Fermi cycle without requiring an additional (external) pre-acceleration mechanism: this is the shock-injection problem. In this review we restrict ourselves 
to a brief discussion of just these two problems. A more complete discussion can be found in Balogh et al. (2009).

\subsection{Self-consistent shock turbulence}

\subsubsection{Semi-analytical theory}

The self-consistent problem of shock-particle acceleration and generation of plasma turbulence was first treated semi-analytically by Lee (1982), who assumed that the fast shock-accelerated particles themselves generate the broad spectrum of Alfvén waves in which they become diffusively accelerated. The relevant instability for this process is the streaming instability of Alfvén waves. Its spectrum saturates quasi-linearly, with the particles experiencing pitch-angle diffusion in the waves. Diffusion in velocity space then translates into spatial diffusion. In this way wave excitation and saturation on the one hand and pitch-angle diffusion and spatial diffusion of energetic ions on the other hand form a closed cycle of acceleration and self-consistent excitation of Alfvénic turbulence.

In equilibrium, diffusive acceleration of ions is governed by the (stationary) convection-diffusion equation

$$
\nabla_{z}\left[\kappa_{\|}(v, z) \nabla_{z} F(v, z)\right]-(2.4 / a)^{2} \kappa_{\perp}(v, z) F(v, z)+V F(v, z)=0
$$

which describes the final shape of the ion distribution $F(v, z)$ in velocity space $v$ at location $z$ under the action of convection $V$, spatial diffusion with parallel and perpendicular coefficients $\kappa_{\|}, \kappa_{\perp}$, which are functions of both velocity and space, and escape from the shock at a prescribed distance $a$. The parallel diffusion coefficient

$$
\kappa_{\|}=\frac{1}{4} v^{2} \int_{-1}^{1} \mathrm{~d} \mu\left(1-\mu^{2}\right)^{2} / D_{\mu \mu}
$$

is related to the pitch-angle diffusion coefficient

$$
D_{\mu \mu}=\left(\pi \omega_{c i}^{2} / 2 B^{2}\right)\left[\left(1-\mu^{2}\right) /|\mu|\right] W_{k}\left(k_{\|}, z\right)
$$

where $W_{k}$ is the spectral energy density of the Alfvén turbulence $\left\langle\left|\mathbf{b}^{2}\right|\right\rangle=\int W_{k} \mathrm{~d} k$ that is in resonance with the ions, i.e. taken at $k_{\|} v=\omega_{c i} / \mu$. The spectral density evolves according to the quasilinear equation

$$
-\left(V \mp V_{A}\right) \nabla_{z} W_{k}=2 \gamma^{A} W_{k}
$$

where $\gamma^{A}$ is the linear growth rate of Alfvén waves. The \pm sign refers to the upstream or downstream wave directions. The time, an ion needs to be accelerated to a given momentum, can be expressed in terms of the shock compression ratio $\rho_{\text {sh }}=N_{2} / N_{1}$ as (Giacalone and Neugebauer 2008) 


$$
\frac{3 \rho_{\mathrm{sh}}}{\left(\rho_{\mathrm{sh}}-1\right)} \frac{\kappa(p)}{s V_{1}^{2}}<\tau_{\mathrm{acc}}(p)<\frac{3 \rho_{\mathrm{sh}}\left(1+\rho_{\mathrm{sh}}\right)}{\left(\rho_{\mathrm{sh}}-1\right)} \frac{\kappa(p)}{s V_{1}^{2}}
$$

on the assumption that $\kappa(p) \propto p^{s}$ in the upstream region is a power law function of the particle momentum $p$. The upper bound is obtained for constant $\kappa$, which corresponds to no additional downstream turbulence. The lower bound follows for zero downstream diffusion coefficient.

\subsubsection{Diffusion coefficient}

This acceleration time is very long, indeed. Except for the simplifications made in this theory, the assumption of stationarity is therefore crucial. In addition the further assumption about the perpendicular diffusion coefficient is that the ion moves just one gyroradius $r_{c i}$ in one diffusion time: $\kappa_{\perp}=r_{c i}^{2} / \tau_{d}$, with velocity $v=\lambda_{\mathrm{mfp}} / \tau_{d}$. Assuming that $\kappa_{\|}=\frac{1}{3} v \lambda_{\text {mfp }}$ is represented by the classical expression through the mean free path $\lambda_{\mathrm{mfp}}$, this yields that

$$
\kappa_{\perp}=v^{4} / 3 \kappa_{\|} \omega_{c i}^{2}
$$

can be expressed through the known parallel diffusion coefficient.

Clearly, there is little reason for the parallel diffusion time to coincide with the displacement in transverse direction by one gyroradius. Rather, in the collisionless state the transverse diffusion is due to non-classical effects which are not covered by quasilinear pitch-angle scatterings. Such a theory has been provided earlier (Shlesinger et al. 1993; Treumann 1997) and shows that under collisionless conditions the parallel diffusion coefficient becomes

$$
\kappa_{\|}(d, t ; v)=\kappa_{\| \mathrm{cl}}[v /(v-d / 2)]\left(v_{\mathrm{an}} t\right)^{\alpha}, \quad \alpha=(4 v-2 d-1)^{-1}
$$

Here $v>3 / 2$ the power of the non-classical interaction probability, and $d$ is the dimension of the problem which is usually $d=3$. This diffusion coefficient depends on time $t$, and the transverse diffusion coefficient becomes time-dependent as well. Again, expressing it through $\kappa_{\|}$yields

$$
\kappa_{\perp}=\left(\frac{v}{v-d / 2}\right)\left(\frac{v}{\omega_{c i}}\right)^{2} \frac{T_{i}}{m_{i} v}\left(v^{2} t\right)^{\alpha}, \quad 0<\alpha<\frac{1}{3}
$$

Only when classical diffusion takes over after a binary collision time does the diffusion coefficient become time independent and the mean square displacement increase linearly with time $t$. Simulations of the particle displacement in shock acceleration (Scholer et al. 2000) exhibit just this behaviour of non-stationary diffusion. In these simulations a power of $\alpha \sim \frac{1}{6}$ was found, which is in excellent agreement with collisionless diffusion theory (Treumann 1997). This diffusion is faster than classical. It consists of waiting periods, when the ion calmly gyrates around the magnetic field, and jumps that are caused by the unpredictable interaction with a passing wave. Such a kind of 'superdiffusion' starts from zero displacement until, at binary collision time, it reaches a value that serves as initial value for classical diffusion. 
Classical diffusion is the long-time average of these subsequent states of collisionless diffusion. Each time, when a binary collision time has passed, the particle experiences a slow down in its motion and starts a new round of collisionless diffusion. Diffusive shock theory in collisionless shocks should account for this kind of behaviour. However, for very long classical (binary) collision times the parallel (70) and perpendicular (74) diffusion coefficients do not hold yet but must be exchanged with the corresponding collisionless diffusion coefficients $(75,76)$.

\subsubsection{Turbulent wave spectra}

The dependence of the shock acceleration process on the wave spectral power density $W_{k}$ is important. It implies that the entire diffusive acceleration process depends crucially on the assumption of which waves are involved. By the nature of the diffusive approximation (the Fokker-Planck equation), these waves must be distributed isotropically in space, a condition that is barely satisfied in the vicinity of curved shock waves such as planetary bow shocks in a high Mach number stream such as the solar wind or stellar winds. If the waves are related to the shock then it is clear that the turbulence will be inhomogeneous. The turbulent power should maximise close to the shock and decay with distance from the shock. This implies that scattering probabilities decrease with increasing distance from the shock.

Upstream of quasi-perpendicular shocks, enhanced wave power is found only in the shock foot region. The transverse size of shock feet is of the order of one thermal upstream ion gyroradius $r_{c i}=v_{i} / \omega_{c i}$. Energetic ions, which enter the upstream region from downstream after having been scattered there into the upstream direction, completely ignore the foot and take it as part of the shock transition which, in shock acceleration theory, is assumed to be infinitesimally narrow. Farther away from the quasi-perpendicular shock, the upstream flow contains weak and undisturbed upstream turbulence. The low upstream turbulence level makes shock acceleration of shock-reflected particles at quasi-perpendicular shocks rather ineffective. Even for the energetic backscattered particles, it requires very large systems of stationary turbulence and very long acceleration times. In the case of the solar wind, the upstream solar wind turbulence is about weak Kolmogorov with spectrum $W_{k} \propto k^{-\frac{5}{3}}$ (Tu and Marsch 1995; Goldstein et al. 1995; Biskamp 2003; Narita et al. 2009) (see also the left part of Fig. 44).

Supercritical quasi-parallel shocks could work more efficiently as particle accelerator machines. They possess a substantial and spatially extended level of upstream wave activity, consisting of low-frequency upstream waves, Alfvén-ion cyclotron waves, whistlers, shocklets and pulsations (SLAMS). They together produce a comparably intense, though anisotropic and inhomogeneous, turbulence (Narita et al. $2006,2009)$ that, in addition, decays in intensity with increasing distance from the shock. The assumption of an isotropic and homogeneous wave distribution upstream of a quasi-parallel shock is, however, barely realistic. Theoretical wave spectra are not very reliable as they cannot account for the entire complexity of the interaction between the diffuse particle component in the wave generation process, the wave cascading and nonlinear interactions that take place, if the power is injected in a certain 


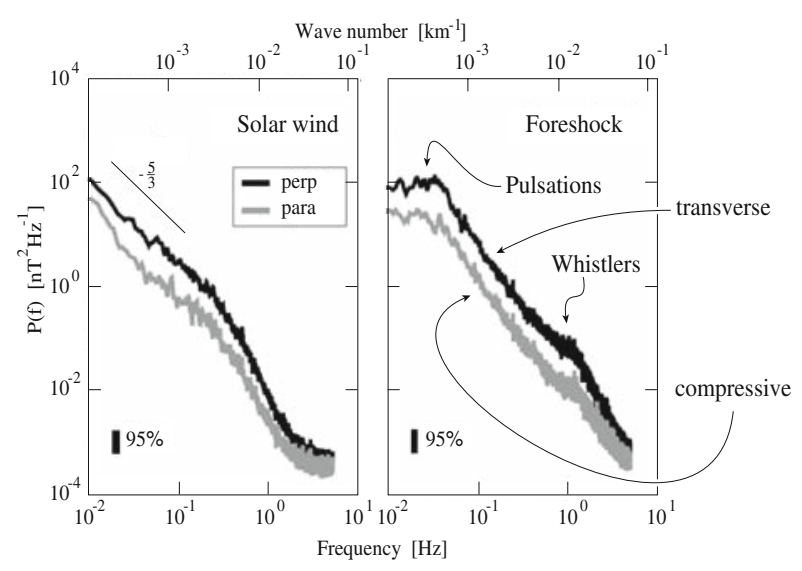

Fig. 44 Magnetic power spectral densities in the solar wind (left) and foreshock (right) versus time $t$ and wave number $k$ for transverse and compressive fluctuations. The solar wind spectra decay like Kolmogorov for low frequencies. Foreshock spectra are flat at low frequencies due to shocklets and pulsations (SLAMS) and have a maximum at $\sim 0.05 \mathrm{~Hz}$ which from pulsations at transverse wavelengths $\lesssim 10^{3} \mathrm{~km}$. At higher frequencies spectra are Kolmogorov though the range of power law is short and the uncertainty might hide a steeper decay (after Narita et al. 2006)

wave number band and reaches high enough values. The best possible way to account for this turbulence is to refer to in situ observations which again can be performed only in the vicinity of Earth. Otherwise, they must be inferred remotely by indirect methods.

Figure 44 shows an example of measurements of the low frequency wave power in the foreshock of Earth's bow shock in comparison to solar wind wave power measurements. In both cases the anisotropy is quite pronounced, with the solar wind at low frequencies/large wavelengths behaving approximately Kolmogorov-like. The foreshock spectrum shows the large contribution of pulsations as well as the contribution of magnetosonic whistler-mode waves. The range of power law is shorter than in the undisturbed solar wind and is also steeper.

From here we boldly conclude that the most efficient accelerators of particles will always be quasi-parallel shocks - unless quasi-perpendicular shocks are embedded into a plasma of high turbulence levels such that many backscattering events can occur in the available time the particle is located in the vicinity of the shock.

\subsection{The shock-injection problem}

The only way to treat the problem of whether or not a shock is capable of generating its own particles having energies high enough to start the Fermi cycle of shock acceleration is by numerical simulation.

\subsubsection{Ion shock surfing}

Since quasi-perpendicular shocks with their shock potential reflect low energy ions out of the upstream thermal ion population, one may expect that this ion reflection 
might be capable of starting the Fermi mechanism under the condition that the ions are not reflected by upstream turbulence but experience scattering in the shock foot waves, i.e. they surf along the shock front (Sagdeev 1966; Sagdeev and Shapiro 1973; Ohsawa 1985a,b; Terasawa et al. 1985; Sugiyama and Terasawa 1999). The reflecting shock electric potential field can be estimated from the electron momentum equation, neglecting the motional electric field, $e \phi(x) \approx \int_{-\infty}^{x} \mathrm{~d} x N^{-1} \nabla_{x}\left(B^{2} / 2 \mu_{0}+P_{e}\right)$. Making use of the constancy of the total pressure $\left[B_{2}^{2} / 2 \mu_{0}+P_{e 2}+m_{i} V_{2}^{2}\right]=0$ and neglecting the electron pressure to first approximation, this can be rewritten, yielding

$$
e \phi(x) \approx \frac{B_{1}}{\mu_{0} N_{1}} \int_{-\infty}^{x} \mathrm{~d} x \frac{\partial B}{\partial x}=\frac{1}{\mathscr{M}_{A}^{2}} \frac{\Delta B(x)}{B_{1}} m_{i} V_{1}^{2}
$$

where $\Delta B(x)=B_{2}(x)-B_{1}$ is the difference between the downstream and upstream magnetic fields taken at position $x$. For ions, this potential implies that ions with energy $\frac{1}{2} m_{i} v_{x}^{2}<e \phi(x)$ will be reflected at position $x$, because the potential is positive. As has been discussed in connection with quasi-perpendicular shocks, these reflected ions are partially responsible for the quasi-periodic reformation of quasi-perpendicular shocks. When gyrating back into the upstream plasma they form the shock foot, retard the inflow, cause a foot current, and experience the motional electric field to become accelerated along the shock surface until gaining sufficient energy to overcome the reflecting shock potential.

The maximum energy gain of the accelerated ions in this multiple upstream reflection before passing the shock, which we can call 'ion surfing', can be estimated from the balance between the Lorentz and electric forces on the ion, yielding a simple upper limit for the ion velocity tangential to the shock, $v_{y, \max } \sim E_{x} / B_{z}$, where $E_{x}$ is the cross-shock reflecting electric field based on the above potential. Combination with the above result then produces the maximum achievable energy gain

$$
\mathscr{E}_{i, \max } \sim \frac{m_{i}}{2} v_{y, \max }^{2} \sim \frac{m_{i}}{2}\left(\frac{1}{\mathscr{M}_{A}^{2}} \frac{m_{i} V_{1}^{2}}{e B_{1} \Delta_{s}} \frac{\Delta B}{B_{1}}\right)^{2}
$$

in this multiple shock-surfing reflection process, where $\Delta_{S}$ is the thickness of the cross shock ramp electric field region.

The maximum energy gain depends crucially on the narrowness of this region. Since the shock thickness is not well known and the effect of the overshoot field is difficult to estimate, one assumes that in the overshoot region the magnetic field can be described approximately by a solitary structure, yielding an estimate $\Delta B / B_{1} \sim 2\left(\mathscr{M}_{A}-1\right)$ for the maximum overshoot amplitude. With this estimate, the maximum energy gain becomes very large

$$
\mathscr{E}_{i, \max } \sim 2 m_{i} V_{A}^{2} \mathscr{M}_{A}^{2}\left(m_{i} / m_{e}\right)=2 m_{i} V_{1}^{2}\left(m_{i} / m_{e}\right)
$$

In fact, in the solar wind the streaming energy of ions is of the order of $1 \mathrm{keV}$, implying that the maximum energy an ion could gain in surfing along the Earth's bow shock 
amounts to the order of $\mathscr{E}_{i, \max } \sim 7.5 \mathrm{MeV}$, which is quite high in this case. This energy would indeed be sufficient for entering the Fermi acceleration cycle, while the observations suggest a steep cut-off of the proton spectrum at the bow shock above a few $100 \mathrm{keV}$. This cut-off could be due to the curvature of the shock that does not allow a proton to surf for a sufficiently long time. But the question remains unresolved whether or not the quasi-perpendicular bow shock — and thus any quasi-perpendicular shock - can by itself accelerate ions in shock-surfing to exceed the energy threshold of the Fermi cycle.

\subsubsection{Terasawa's mechanism of ion surfing}

An attempt of finding a mechanism of ion-shock surfing in order to extract ions from the thermal particle population Sugiyama and Terasawa (1999) suggests that ions could be trapped in a large amplitude upstream wave when the wave is crossing the shock. The mechanism exploits the fact that the flow velocity of an upstream (monochromatic) wave decreases when the wave passes the shock. The velocity of upstream waves is essentially the Alfvén speed. During the passage time of the wave across the narrow shock transition, the trapped particle bounces back and forth across the shock transition between a fast and a slow propagating wave, in this case, and has a chance to pick up the wave phase-velocity differences. The particle moves along lines of constant energy when jumping from one frame to the other. This process gradually increases the particle energy. For a high bounce frequency of the particle it experiences many reflections and can thus be speeded up to an energy, which might become high enough for injection into the Fermi mechanism. The action of the mechanism is schematically shown for a monochromatic wave in Fig. 45. This mechanism has been elaborated by Sugiyama and Terasawa (1999) and Sugiyama et al. (2001). The particle orbit and energy gain is shown on the right in the figure. The process is essentially diffusion in energy space, which for sufficiently large wave amplitudes becomes chaotic. The stepwise increase in particle energy is well illustrated.

A severe restriction is the assumption of monochromatic waves. Simulations have shown that quasi-parallel shocks are built of large-amplitude and irregularly-shaped pulsations. These pulsation propagate at fast speeds before becoming the shock, i.e. before arriving at the shock location. Ions are not only retarded but also trapped between the pulsations and the old shock front. Close to the shock, during the approach of the pulsation, they may bounce back and forth many times between the old and new shock, a process similar to Terasawa's acceleration, though different in the sense as no plane wave exists in this case and, in addition, the trapped ions do not cross the shock but experience the upstream convection electric field. The ions that are trapped in this way bounce between two approaching magnetic mirrors, thereby experiencing an adiabatic acceleration in the perpendicular direction. Since close to the shock transition, the shock normal has turned to become quasi-perpendicular, the ions in addition feel trapped in kind of a shock foot where they become accelerated along the convection electric field in the direction tangential to the shock ramp over the distance of the approaching new pulsation. Both these types of acceleration raise the ion energy and at the same time isotropise the ions. The result is that the group of trapped ions becomes isotropically accelerated. This mechanism has not yet been evaluated, but it 

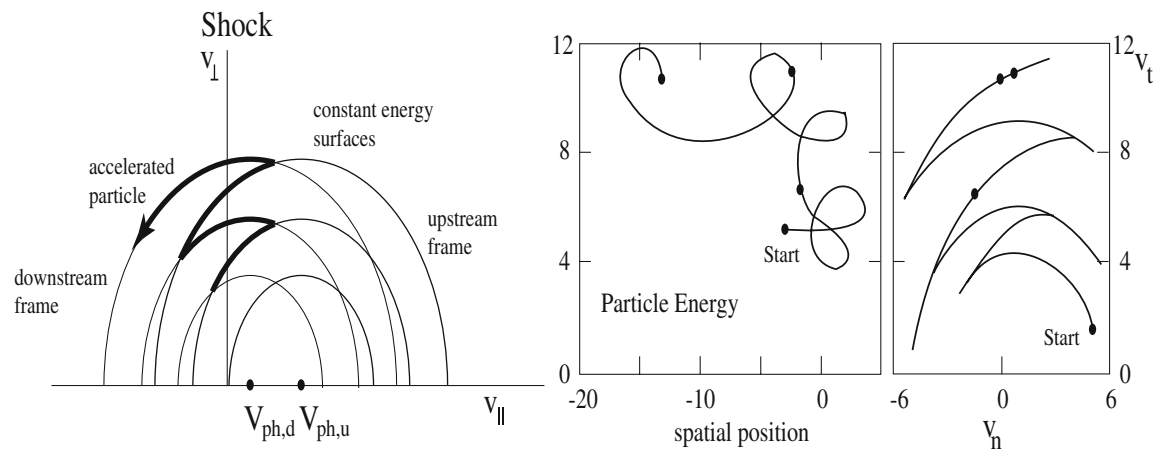

Fig. 45 Particle injection from a quasi-parallel shock by trapping acceleration across the shock (after Sugiyama et al. 2001). Left: Schematic of the mechanism. The upstream and downstream waves move at different phase velocities. The curves show circles of constant energy for a trapped particle. The ion moving back and forth across the shock along the different lines picks up the energy between the upstream and downstream wave frames exactly like in Fermi acceleration, until having sufficient energy to escape and enter the real Fermi process. Right: Particle orbits in this process. The energy increases while the particle is moving with the waves. The outer panel shows the bouncing in normal and tangential velocity coordinates

is suggestive of quasi-parallel shock acceleration of ions to build a diffuse energetic ion component which has its source at the quasi-parallel shock.

\subsubsection{Hybrid simulations of shock particle acceleration}

One-dimensional and two-dimensional hybrid simulations of self-consistent shock formation, with the focus on particle acceleration, have been performed for roughly the past two decades with increasing resolution (Burgess 1987; Kucharek and Scholer 1991; Scholer and Kucharek 1999a; Giacalone 2004, 2005; and others).

Reconstruction of observed AMPTE IRM ion energy spectra, using self-consistent 1D hybrid simulations of a quasi-parallel $\theta_{B n}=30^{\circ}$ shock (Scholer and Kucharek 1999b), yield information about the possible self-consistent ion-injection mechanism. Since the amount of self-consistently produced shock-accelerated ions in the simulations was very small, these authors applied the trick of splitting each reflected ion into 30 parts, thereby generating a factor of 30 more reflected ions than were produced in the simulation, while not changing the total charge and mass of the ion component, i.e. distributing only a fractional charge to each of the split ions. Splitting was applied only to reflected ions. The upstream boundary of particle escape was assumed fixed in space, causing the distance of the shock-escape boundary to shrink continuously during outward shock propagation. In addition, a finite resistivity had to be assumed making the simulations not fully collisionless. Figure 46 shows a pure proton-simulation run (left) and protons and helium nuclei (centre and right) compared to observations. The fits of the simulated and observed data are satisfactory for both the upstream and downstream particle energy fluxes. Exponential particle energy spectra are obtained (Kucharek and Scholer 1991; Scholer and Kucharek 1999a).

The two flux curves in the left panel belong to the total upstream proton flux, which consists of the Maxwellian flow and a shock-accelerated (by the splitting artificially enhanced in particle number) exponential bump on the tail of the distribution that is 


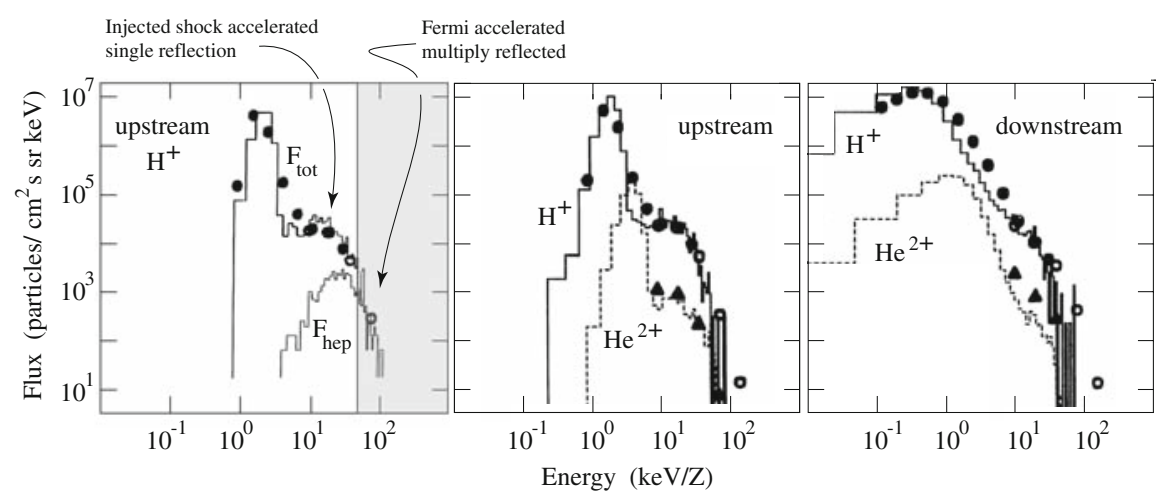

Fig. 46 1D-hybrid simulations with ion splitting showing the acceleration of ions across the injection boundary for multiple reflection (first order Fermi) acceleration (data from Scholer and Kucharek 1999b, courtesy American Geophysical Union). Left: The simulated upstream proton spectrum compared with the observations. The total proton flux $F_{\text {tot }}$ agrees satisfactorily well with the observations. The distribution exhibits the Maxwellian upstream flow and a broad shock-accelerated exponential bump at energies $>10$ $\mathrm{keV}$, typical for shock acceleration. The distribution $F_{\text {hep }}$ contains the population that has been accelerated by the shock but is scattered back towards the shock thus becoming multiply scattered. Above $\sim 50 \mathrm{keV}$ it makes up the total distribution identifying the shaded region as the Fermi acceleration domain. Centre: Similar upstream simulation for protons and Helium nuclei. Right: The downstream distribution for the two particle components fitted to the observations. The agreement between observation and simulation is reasonable

self-consistently produced by the shock. Plotted is the distribution $F_{\text {hep }}$ of those particles which had been scattered back towards the shock from upstream and will, after reaching the shock, have another chance to be reflected another time and accelerated further. In fact, above $\sim 50 \mathrm{keV}$ (in these simulations which have been tailored for bow shock conditions) the total high energy flux is completely built up of these multiply reflected particles. Hence this is the boundary of the domain of first order Fermi acceleration where the particles become accelerated. Thus it seems that quasi-parallel shocks are capable of generating a low-density seed population for first-order Fermi acceleration. ${ }^{29}$ Further simulations showed that the shock-generated ion spectrum extends into the energy range of a few ten times the upstream ram flow energy.

This is a conclusion of vital importance in application to astrophysics. However, the mechanism of how the parallel shock is able to accelerate the particles in order to inject them into the Fermi cycle still remains an open problem. It is not answered by these simulations except that they show that the energetic particle component is not a population that is leaking out from the downstream region into the upstream region. In similar simulations in one dimension but a much larger spatial simulation domain $x \geq 28,000 \lambda_{i}$ and simulation times $t \omega_{c i}=4,000$ (Giacalone 2004, 2005) for a parallel shock of Mach number $\mathscr{M}_{A}=6.4$ the differential energy flux spectrum exhibits a shoulder at high energies. Because of the large simulation domain and long

\footnotetext{
${ }^{29}$ It must be stressed that the extension of the bump distribution into the Fermi domain becomes detectable solely due to the particle splitting procedure, which artificially increases the number of accelerated particles and, in addition, allows for reasonable statistics. Clearly, even though the number of charges is not changed by this process, the increase in particle number may have had an uncontrollable effect on the shock.
} 

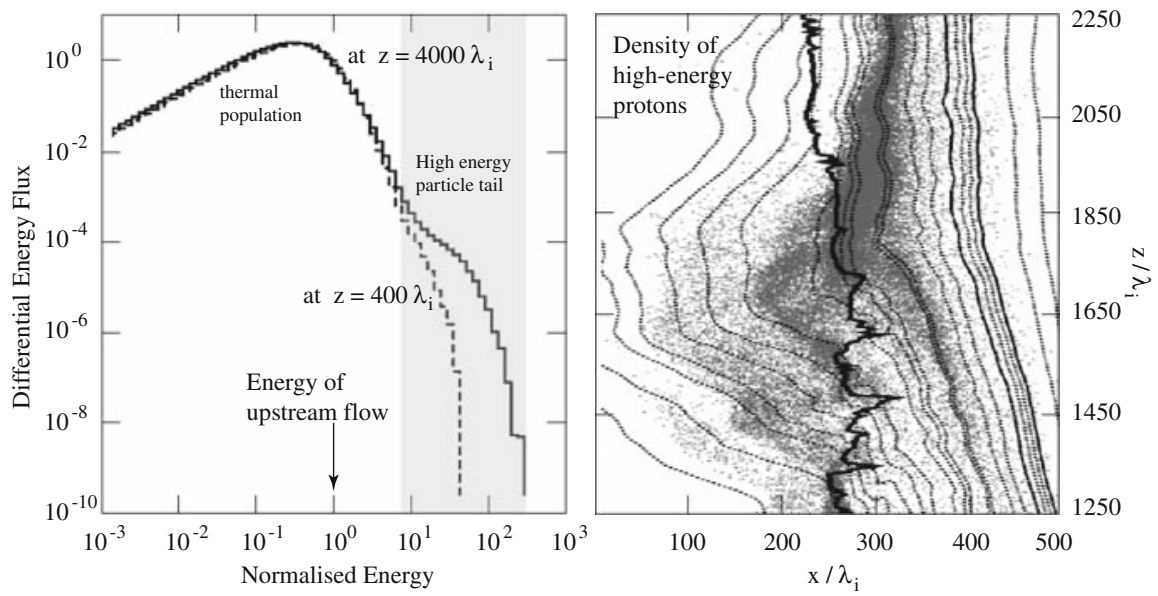

Fig. 47 2D-semi-self-consistent extended simulation box simulations of proton acceleration at a nearly perpendicular shock (after Giacalone 2005). Left: Spectra at two different locations. The high energy tail that has evolved at the distribution is more expressed as the distant location. Right: The energetic proton distribution in the $x, z$-plane and the field lines around the shock at time $t \omega_{c i}=150$. Plotted are only the protons in the tails of the distribution (shaded on the left at two locations). The shock ramp is the dark line, the field lines are the light lines. Energetic particles concentrate almost exclusively inside the looping magnetic field lines. It seems that here the strongest acceleration takes place

simulation times, these shoulders extend out to energies up to 200 times the initial upstream plasma ram energy (in application to the solar wind an acceleration up to $200 \mathrm{keV}$ ). Extrapolating to SNRs where the upstream flow energy may reach energies of $\sim 10 \mathrm{MeV}$, one may expect particle acceleration up to a few $\mathrm{GeV}$.

Semi-selfconsistent 2D simulations at Mach number $\mathscr{M}_{A}=4$ (Giacalone 2005) at a perpendicular shock are shown in Fig. 47. In this case a turbulent magnetic wave spectrum was superimposed on the magnetic field with spectral index $\varsigma=\frac{5}{3}$ simulating Kolmogorov wave turbulence. The spatial extension was $\Delta z=1,000 \lambda_{i}$ along the mean magnetic field, including long wavelength magnetic perturbations. Due to the presence of these waves the shock surface becomes wavy and rippled. It is located at the dark line that undulates between $x=200 \lambda_{i}$ and $x=300 \lambda_{i}$ along $z$. Energetic particles with energy $>10 \times \frac{1}{2} m_{i} V_{1}^{2}$, i.e. larger than ten times the upstream ram energy are plotted. Their upstream density follows the magnetic field, which forms a large loop whose both ends are tied to the shock at two different locations in $z$.

The flux of suprathermal ions is nonuniformly distributed over the shock surface. Specularly reflected ions become accelerated in these magnetic loops due to bouncing back and forth from the shock along the magnetic field not needing any upstream reflection. The particles are acceleration in the upstream motional electric field. This mechanism is independent of the presence of an upstream turbulence. It should work when the shock-foot magnetic field in two or three dimensions evolves an upstream magnetic-loop structure. In the case of high Mach numbers the pressure of the many reflected ions will self-consistently cause such large upstream magnetic loops. Such loops cannot be seen in one-dimensional simulations. 
The left part in Fig. 47 gives the particle differential energy flux as function of energy normalised to the upstream flow energy. Two spectra are shown that have been taken in the interval $335<x / \lambda_{i}<490$ but for different domain sizes $z_{\max }=\lambda_{\text {corr }}$ in $z$. A high energy population - up to energies of 100 times the upstream flow energy -is produced. The spectra are very steep, however, and it requires very large correlation lengths and extensions of the shock in the second dimension, in order to flatten them substantially. Nevertheless, this simulation suggests the possibility for accelerating particles to high energies at perpendicular shocks out of the thermal distribution, which is of considerable interest for astrophysical application. These ideas can directly be applied to the heliospheric termination shock ${ }^{30}$ where measurements became available only recently.

The theoretical prediction yields an injection threshold energy in the interval $9 \mathscr{E}_{u}<\mathscr{E}_{\mathrm{F}-\text { inj }}<18 \mathscr{E}_{u}$, where $\mathscr{E}_{u}=\frac{1}{2} m_{i} V_{1}^{2}$ is the upstream flow energy in the shock frame. In the solar wind in the Earth's bow shock frame we have $\mathscr{E}_{u} \approx 1 \mathrm{keV}$. Hence, in the solar wind the injection threshold should be somewhere between $10 \mathrm{keV}$ and 20 $\mathrm{keV}$, depending on the quasi-parallel or quasi-perpendicular parts of the shock. The simulations in Fig. 46 show that the threshold lies near an energy of $\sim 50 \mathrm{keV}$ which is not in disagreement with the prediction. The bow shock is capable of producing ions in this range. The more optimistic two-dimensional semi-selfconsistent simulations suggest a somewhat lower threshold that, however, should be taken with care.

\subsection{Shock acceleration of electrons}

Since electrons are responsible for the generation of radiation, the acceleration of electrons is of primary importance in astrophysical shocks. One may argue that for long enough times the energy exchange between electrons and ions ultimately leads to isothermalisation of the plasma causing heating of the electrons until they become visible in radiation. However, under truly collisionless conditions the distances of isothermalisation are huge compared with the scales of the shock. Hence, direct acceleration of electrons poses a problem. Because of the direction of the cross-shock electric field which accelerates electrons downstream, electron reflection from a shock is not electrostatic and different from ion reflection. Moreover, Fermi acceleration of electrons is highly reduced due to the low electron mass which implies that electrons behave adiabatically in the upstream and downstream large amplitude low frequency turbulence.

Electron acceleration in non-relativistic shocks meets with two severe problems. The first problem is that a super-critical shock, which must reflect ions in order to get rid of the excess energy, develops a positive shock potential. A simple explanation for its generation in a high Mach number flow at the quasi-perpendicular shock transition can be based on the large discrepancy between the convective electron and ion gyroradii: at same perpendicular speed we have $r_{c e} / r_{c i}=m_{e} / m_{i}$, with the consequence that ions penetrate considerably deeper into the shock transition than electrons. The difference in penetration depth generates a positive space charge over a fraction of the

\footnotetext{
$\overline{30}$ See the Conclusions section below.
} 
distance $r_{c i}$ (or $\lambda_{i}$ ) and gives rise to an upstream directed electric field, which accelerates electrons downstream and inhibits electron reflection. For the inertial lengths we have $\lambda_{i} / \lambda_{e}=\sqrt{m_{i} / m_{e}}$.

The second problem is related to the small electron gyroradius (or inertial length) because it restricts the shock normal angle of electron reflection to near perpendicular. Another difficulty is that for electrons in a stream such as the solar wind, the kinetic energy of the streaming motion $\frac{1}{2} m_{e} V^{2} \ll T_{e}$ is usually much less than the electron thermal energy. At a streaming velocity of $V=1,000 \mathrm{~km} \mathrm{~s}^{-1}$ the kinetic energy is just $\sim 5 \mathrm{eV}$, while the solar wind electron temperature is $T_{e} \simeq 100 \mathrm{eV}$. Even though the shock is supercritical, the dominant electron speed is the electron thermal velocity. Writing $r_{c e}^{2} / r_{c i}^{2}=\left(m_{e} / m_{i}\right)\left(T_{e} / K_{i}\right)$, with $K_{i}=m_{i} V_{i}^{2} / 2$ the ion kinetic energy, we have $r_{c e} / r_{c i}=\sqrt{T_{e} / K_{i}}\left(\lambda_{e} / \lambda_{i}\right)$. In the solar wind $K_{i} \sim 1 \mathrm{keV}$. Hence, due to the large electron temperature, $r_{c e} / r_{c i} \sim \sqrt{m_{e} / 10 m_{i}}$ is just a factor of 3 smaller than the ratio of the inertial lengths. However, the high electron temperature means that the electrons are about isotropic with negligible flow speed, and flow-dependent electron reflection per se as considered in the next section does practically not depend on the flow but only on the electron temperature.

\subsubsection{The Sonnerup-Wu electron reflection mechanism}

In a purely kinematic picture, the most simple reasoning (Sonnerup 1969; Wu 1984) to explain electron reflection from a nearly perpendicular shock makes use of the energy gain of a shock-reflected particle in the de Hoffmann-Teller frame which moves at velocity

$$
\mathbf{V}_{\mathrm{dHT}}=\left(\mathbf{n} \times \mathbf{V}_{1} \times \mathbf{B}_{1}\right) / B_{1 n}, B_{1 n} \equiv \mathbf{n} \cdot \mathbf{B}_{1}
$$

where $\mathbf{n}$ is the shock normal. In this frame the shock velocity along the upstream magnetic field is

$$
V_{s \|}=V_{\mathrm{dHT}} \sin \theta_{B n}+V_{1 \|}
$$

At a nearly perpendicular supercritical shock, the parallel flow speed $V_{1 \|}$ is small. Particle reflection is then a pure magnetic mirror effect in the converging magnetic field lines at the shock. It can be treated in close similarity to particle mirroring in a magnetic mirror configuration, where particles are mirror-reflected, when their pitchangles against the magnetic field exceed the loss-cone angle.

The particle distribution in the de Hoffmann-Teller frame follows by subtracting $V_{s \|}$ from the parallel upstream particle speed $v_{\|}$. Assuming that a particle can be mirror-reflected at the shock under conservation of the particle magnetic moment, implies that the particle velocity measured in the de Hoffmann-Teller frame has a pitch-angle larger than the loss-cone angle $\alpha>\alpha_{\mathrm{lc}}$, defined through $\sin ^{2} \alpha_{\mathrm{lc}}=B_{1} / B_{o s}$, where $B_{o s}$ is the overshoot (or maximum) magnetic field in the quasi-perpendicular shock transition (Fig. 48). The loss-cone angle is determined through the compression ratio of the shock. For a compression ratio of 3 it is $\alpha_{\mathrm{lc}}=35^{\circ}$. The narrowest loss cone based on the Rankine-Hugoniot MHD relations would be obtained for the largest 

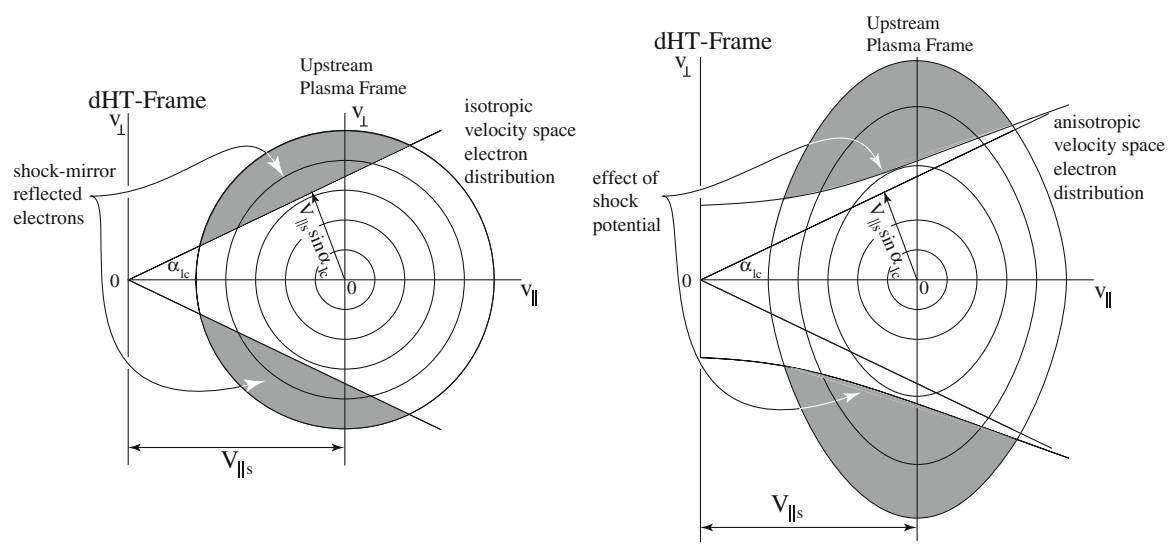

Fig. 48 Electron phase space distribution in velocity space seen from two frames, the upstream plasma frame and the de Hoffmann-Teller frame, on the left the isotropic distribution (after Wu 1984), on the right the anisotropic distribution with $T_{\perp}>T_{\|}$. The dHT-frame is obtained translating the parallel flow velocity in the direction along the upstream magnetic field by the parallel shock velocity $V_{\| s}$. The loss-cone angle seen from the dHT-frame cuts out the particles of low speed which pass the shock. The higher energy particles (shaded) are 'trapped' in the upstream flow magnetic field and can become reflected. On the right the enlarging effect of the attractive ambipolar electric shock potential on the loss cone is shown schematically. For being mirror reflected, electrons require quite a large temperature or perpendicular anisotropy

(fluid) compression ratio 4 , just yielding $\alpha_{\mathrm{lc}}=30^{\circ}$. The number density of reflected particles follows from

$$
N_{\text {refl }}=2 \pi \int_{0}^{\infty} \mathrm{d} v_{\|} \int_{v_{\|}}^{\infty} v_{\perp} \mathrm{d} v_{\perp} F_{e}\left(v_{\|}, v_{\perp}\right)
$$

with the distribution taken in the de Hoffmann-Teller frame. It is clear that electron reflection takes place only if the distribution is hot.

As long as sufficiently high energy electrons are present in the upstream plasma, some of them will become reflected due to the action of the 'shock mirror force' and will return into the upstream flow where they experience the upstream electric induction field and become accelerated in the direction opposite to the reflected ions. This is shown by the shaded regions of the distributions outside the loss-cone ${ }^{31}$ in Fig. 48. thereby increasing the current in the foot of the quasi-perpendicular shock. This perpendicular acceleration further increases the electron energy and thus increases the chance of the electrons to be reflected a second time. In addition it introduces an anisotropy (see the right part of the figure) into the accelerated electron distribution

\footnotetext{
31 One should note that this reasoning holds for both kinds of particles, ions and electrons. It is completely independent of any electric shock potential. For ion reflection it increases the probability of an ion of being reflected and adds to the reflecting electrostatic field. For electrons, the mirror effect acts against the shock-electric field, which wants to accelerate electrons downstream. Hence, including a shock potential, effectively widens the loss cone. This effect is, however, not strong for electrons, because of the large electron temperature (in the solar wind $T_{e} \sim 100 \mathrm{eV}$ ).
} 
Fig. 49 Sketch of electron shock surfing (after Hoshino 2001). The electron after arriving at the shock becomes trapped for a while inside the shock potential which in this case is assumed to be a solitary wave. After having become accelerated the electron leaves the shock downstream

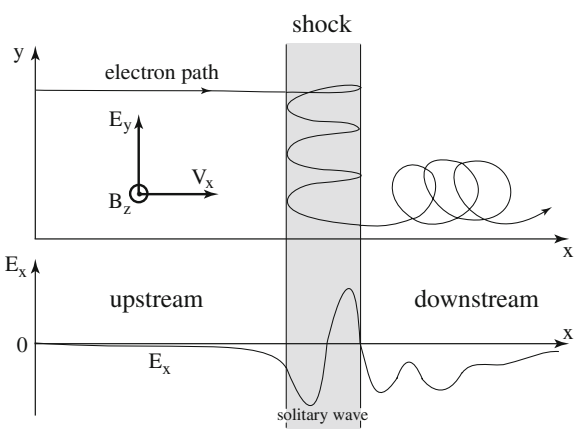

with higher perpendicular than parallel energy, elongating the electron distribution into the perpendicular direction, which is in favour of the reflectivity.

\subsubsection{Electron shock surfing mechanism}

The magnetic mirror-reflection mechanism neglects any electric potential drop in the shock transition. There are several contributions to such a potential drop. The first is due to the inertia difference between ions and electrons, which induces the general ambipolar large-scale shock electric field which we have discussed earlier in relation to ion shock surfing. Electrons are attracted by this potential. However, the intensification of the current in the shock front by the mirror effect can cause several instabilities to grow and can substantially modify the structure of the electric field such that electrons can, under certain conditions, also become reflected in electric wave fields, thereby intensifying the mirror reflection process, ${ }^{32}$ an idea that has been made use of in electron shock acceleration simulations (Hoshino 2001; Hoshino and Shimada 2002; Amano and Hoshino 2007). The passing electron population is heated and thermalized by the action of micro-instabilities in the shock transition layer (Fig. 49), since electrons are most vulnerable to micro-instabilities.

Recent observations of large amplitude highly localised electric fields in shock transitions in space (Bale et al. 2002; Behlke et al. 2003, 2004; Hull et al. 2006; Oka et al. 2006; Bale and Mozer 2007) support this claim. According to these observation, the shock transition is a region where large amplitude solitary structures (or BGK modes) are generated. The most recent measurements by the Polar spacecraft (Bale and Mozer 2007) in Earth's bow shock suggest parallel electric fields $E_{\|} \lesssim 100 \mathrm{mV} / \mathrm{m}$, and perpendicular electric fields $E_{\perp} \lesssim 600 \mathrm{mV} / \mathrm{m}$ on parallel scales comparable to the electron inertial length $\lambda_{e}$. Such fields correspond to localised electrostatic potentials of $\Delta \phi \sim$ several $100 \mathrm{~V}$ along the magnetic field and $\lesssim 1 \mathrm{kV}$ perpendicular to the ambient shock magnetic field.

Production of these structures requires the inclusion of electron dynamics and refers to nonlinear kinetic plasma theory. It is thus quite natural to assume that such structures are accompanied by electron acceleration and heating, in particular as, on these

\footnotetext{
32 If this happens, not only reflection back upstream becomes possible, but also energetic electron injection downstream, as electron acceleration by wave processes is not necessarily a directional effect.
} 
short scales, the electron magnetic moment is not conserved anymore and the electrons become effectively non-magnetic and vulnerable to prompt acceleration in the parallel and also in the perpendicular electric fields. The corresponding potential drops are proportional to the square of the Mach number. Thus, in application to astrophysical shocks, one expects that

$$
\Delta \phi \sim 10\left(\mathscr{M} / \mathscr{M}_{\mathrm{SW}}\right)^{2} \mathrm{~V}
$$

where $\mathscr{M}_{S W} \sim 8$ is the canonical solar wind Mach number. An astrophysical Mach number of, say, $\mathscr{M} \sim 10^{3}$ could thus cause potential drops the order of $\Delta \phi \sim$ several $10^{2} \mathrm{kV}$.

Considering the presence of the small-scale electric field structures, which are solitary structures of the family of BGK modes of spatial scales $\lambda_{e} \sim(10-100) \lambda_{D}$, several Debye lengths long, has two additional interesting aspects. Firstly, these solitary structures expel electrons from their interiors, i.e. they represent localised negative potentials which act repulsing on the electrons. Hence, while the charge separation in the shock front - that previously has been mentioned as causing a problem in electron reflection-accelerates electrons downstream, the localised solitary structures compensate and overcompensate for this effect. Electrons can in this way become effectively reflected from the shock in spite of the shock ramp bearing a larger scale electron-attracting potential. On the other hand, passing electrons experience an additional downstream acceleration.

Secondly, electrons have a chance of being reflected only when impacting on one of these solitary structures and feeling the solitary wave field. It is just the fraction of upstream electrons that collide with the solitary structures that is reflected. This explains why not all particles of smaller than maximum reflection energy will be reflected from the shock ramp. The particle should, in addition, collide with the solitary structure. For efficient acceleration, it should also become trapped. This is possible, in principle, in two ways: by entering the solitary structure in which it can become trapped, because these BGK modes are positively charged and lack electrons inside. For this to happen, the electron must overcome the negative wall around the BGK mode. ${ }^{33}$ It can also be done by jumping from one solitary BGK structure to the next in coherent interaction with the BGK modes for some limited time.

Figure 49 suggests that both types of reflected/trapped electrons experience the upstream motional electric field and are accelerated along the shock front. This increases the reflected particle current along the shock front. This current becomes striated consisting of a distributed shock foot current which contains much stronger current filaments in those narrow regions where the reflected electrons flow along the shock. The energy gained by an electron follows from

$$
\dot{p}_{x}=-e E_{x}-e v_{y} B_{z}, \quad \text { with } p_{x}=m_{e} \gamma v_{x}
$$

\footnotetext{
33 The BGK mode is a quadrupolar structure that is built of the bipolar interior electric field and the reflecting negative electron wall around the BGK electron phase-space hole; often only the bipolar structure is seen when the wall electrons are convected away.
} 
and $E_{x}$ the amplitude of the BGK mode. Acceleration works as long as $e E_{x}>e v_{y} B_{z}$. During the nonadiabatic phase feeling $E_{y}$, the velocity $v_{y}$ increases until this inequality inverts and the electron escapes from the BGK mode soliton. The energy it can reach in this case follows from the condition that the two forces have equal magnitude, or $v_{y}=E_{x} / B_{z}$. Estimating this quantity requires knowledge of the solitary wave amplitude. Since in the BTS instability the BGK modes take their energy from the electron-ion current,

$$
\epsilon_{0} E_{x}^{2} \sim \zeta m_{e} N v_{d}^{2}
$$

where $v_{d} \sim 2 V_{1}$ is the current drift velocity, ${ }^{34}$ which is assumed to be responsible for the Buneman instability. The factor $\zeta$ is the conversion efficiency which, from Buneman instability theory, is taken as $\zeta \sim \frac{1}{4} \approx\left(m_{e} / m_{i}\right)^{\frac{1}{3}}$. Using $E_{1}=V_{1} B_{1}$ the above expression yields a BGK soliton amplitude

$$
\frac{E_{x}}{E_{1}}=\frac{2 c}{V_{1 A}}\left(\frac{\alpha m_{e}}{m_{i}}\right)^{\frac{1}{2}} \simeq \frac{2 c}{V_{1 A}}\left(\frac{m_{e}}{m_{i}}\right)^{\frac{2}{3}}
$$

In the solar wind the Alfvén velocity is between $50<V_{1 A}<150 \mathrm{~km} / \mathrm{s}$ yielding $15<E_{x} / E_{1}<45$, which corresponds to amplitudes of $E_{x}<500 \mathrm{mV} / \mathrm{m}$ well in the range of observations in near Earth space.

The maximum energy the electron can attain is obtained from the equilibrium between the electric and Lorentz forces, yielding $v_{y}^{\max } / c \lesssim 2 \mathscr{M}_{A}\left(m_{e} / m_{i}\right)^{\frac{2}{3}}$ and

$$
\frac{\mathscr{E}_{e} \max }{m_{e} c^{2}} \lesssim 2 \mathscr{M}_{A}^{2}\left(\frac{m_{e}}{m_{i}}\right)^{\frac{4}{3}} \sim 10^{-4} \mathscr{M}_{A}^{2}
$$

For medium Mach numbers of the order of, say, $\mathscr{M}_{A} \sim 10$, electrons can gain a maximum energy of a few per cent of their rest energy, which is in the $\sim 10 \mathrm{keV}$ range. This should, in principle, be sufficient for entering the electron-Fermi cycle in the solar wind.

On the other hand, for very large Mach numbers the possibility arises that the electrons remain trapped for very long time in the BGK solitary structure, in which case the inequality is inverted, and the electron may gain energy quite far in excess of its rest energy. This will happen for Mach numbers $\mathscr{M}_{A}>100$, cases that are realised in astrophysical systems.

\subsubsection{Detailed electron dynamics}

Several increasingly sophisticated numerical simulations have been performed in order to check the proposal of the electron shock surfing mechanism. The question arises which instability is responsible for the generation of the solitary structures which could

\footnotetext{
34 The current drift is the difference between the reflected ions and the inflowing electrons, thus for specular reflection of ions and passing electrons it is just twice the upstream flow velocity.
} 
reflect and trap electrons. There are a number of instability candidates which we have discussed in the section on quasi-perpendicular shocks. These are the BTS instability, the MTSI, and the ion sound instability.

The Buneman instability requires electron thermal speeds smaller than the electronion current drift speed $v_{d}=v_{d i}-v_{d e} \approx-v_{d e}>v_{e t h}$. Taking the reflected ions and incoming electrons, it can marginally grow with growth rate $\sim 0.03 \omega_{p e}$. The Buneman instability immediately heats the electrons until the increase in electron temperature interrupts its growth by letting the drift velocity drop below thermal speed. It, however, quickly forms the required BGK modes which reflect low energy electrons, and also traps some of them. The heated electrons lie outside the de Hoffmann-Teller loss cone and can become mirror reflected. ${ }^{35}$ The reflected electrons are further accelerated in the foot, thereby increasing the foot current until this current drives the MTSI.

However, the electron current-driven MTSI is a stronger instability (Matsukiyo and Scholer 2003, 2006a,b), growing faster than the ion cyclotron frequency, and in addition has a lower threshold than the BTS and ion acoustic instabilities. Because it has a parallel component of the wave electric field, It heats electrons parallel to the magnetic field while also producing BGK phase space holes. The situation is quite complex, switching between the different reflected and inflowing species, their densities and temperatures and the angles to the magnetic and electric fields. Only simulations can help understanding the acceleration of electrons.

Figure 20 shows the final electron distribution function (differential energy flux) in a high Mach number $\left(\mathscr{M}_{A}=32\right)$ PIC simulation for a quasi-perpendicular shock. This high Mach number simulation required that the mass ratio was unrealistically set to $m_{i} / m_{e}=20$. The distribution shows the evolution of a long nonthermal tail. The dotted line is the corresponding Maxwellian. At energies $\gamma / \gamma_{0}<2$ the distribution is exponential ( $\gamma_{0}$ is the relativistic energy of the incident flow electrons). At higher energy $\gamma$ it deviates, becoming non-exponential. In a log-log representation the high energy tail is power law $\propto \gamma^{-\alpha}$ with power $\alpha \sim 2$ or larger. It confirms the acceleration of electrons by the action of the Buneman instability, in this case.

In the interaction with a single BGK mode the accelerated electrons cannot gain more energy than $\gamma / \gamma_{0}=1.26$. Acceleration to higher energies requires shock surfing. The BGK modes serve the reflection of electrons including some pre-acceleration. The path of an electron along the shock is shown in Fig. 50. On the left of this figure, the time evolution of the shock normal electric field component $E_{x}$ is given. This field exhibits a quasi-periodic structure in the foot of the shock. The field concentrates in narrow spatial regions which move in upstream direction, turn around and propagate downstream until becoming damped. Two selected particle orbits have been superimposed on the field, one of the particles not being affected by the presence of the electric field (electron 1), and another particle that interacts with the wave electric field (electron 2). Both electrons enter the shock with the upstream convective flow velocity along the straight line at the bottom of the figure. Electron 1 does not feel the wave electric field because of some obscure reason when encountering the shock. It passes over the crest of $E_{x}$. In the back of the electric field it starts performing an oscillatory

35 This was discussed in the section on the Sonnerup-Wu mechanism. 


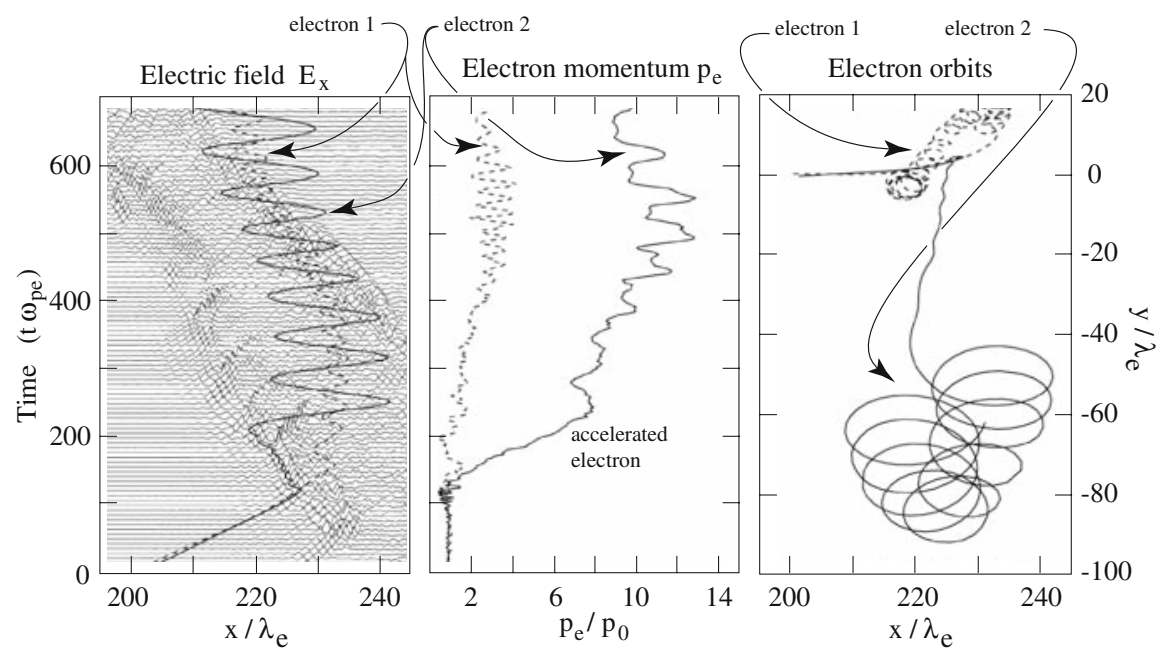

Fig. 50 High Mach number $\left(\mathscr{M}_{A}=32\right)$ electron shock surfing PIC simulation for quasi-perpendicular shocks, showing Left: the time-stacked evolution of the electric wave field $E_{x}$ in the shock transition with two electron orbits overlaid. The dashed orbit belongs to an electron not in resonance with the wave, the solid line is the resonant electron. In contrast to electron 1, electron 2 performs large excursions around the shock position before leaving the shock. Electron 1 also moves with the shock but crosses it without gaining energy. Center: Evolution of the total momentum of the two electrons in time. Electron 1 gains very little momentum/energy after entering the shock, while electron 2 Initially gains very much momentum and then enters a nonlinear state where the gain is slower. Right: The two electron orbits in the $(x, y)$-plane. Electron 1 moves only a small distance in both $x$ and $y$, while electron 2 performs a long initial jump in $y$ at constant $x$ after which it becomes trapped in the wave and bounces back and force with its enlarged gyroradius (after Hoshino and Shimada 2002)

motion as it is now stopped, becoming a slowly moving member of the downstream flow. The central panel shows that its energy gain is small due to adiabatic heating only, while the right panel shows that it has been moderately heated in perpendicular direction when performing its gyrational motion.

Unlike electron 1, electron 2 when encountering the repelling electric field around about time $t \omega_{p e} \sim 100$ is stopped becoming reverted into $-x$ direction. For roughly 100 plasma periods it stays trapped in the electric field following its upstream path. The central panel shows that during this time it becomes violently accelerated by about eightfold times. Having gained that much energy it manages to escape the field and pass downstream now performing large oscillation in $x$ there. From the 2D-representation of its orbit it is taken that, similar to electron 1, it has escaped the electric field but has been shifted a long distance along the shock in the $y$ direction. The oscillations in $x$ seen in the left panel unmask as being projections of its gyro-orbit. Electron 2 has been accelerated nonadiabatically perpendicular to the magnetic field during its contact with the localised electric field. The lack in acceleration of electron 1 can thus be interpreted such that electron 2 has encountered the shock at a position $y$ lacking a wave electric field. The electric field must hence have been highly localised. In fact, from the right panel one realises that electron 1 encountered the shock at $y \sim 10 \lambda_{e}$ only. It is also interesting to see from the right panel that both electrons have not moved far downstream during the time interval of the simulation shown. They are hanging 


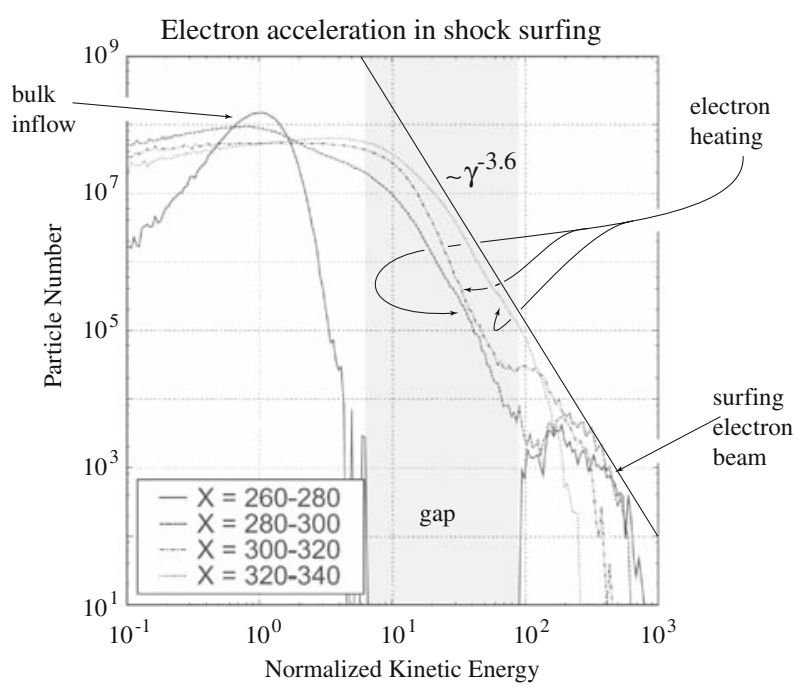

Fig. 51 Spatial evolution of electron energy spectra in electron acceleration by shock surfing (after Amano and Hoshino 2007). Progressing from the upstream foot boundary into the shock the electron spectrum widens with increasing electron temperature and in the centre of the shock evolves from an exponential into a distribution that over a certain energy interval exhibits a clear power law shape. Note the gap between the surfing beam and the bulk inflow distribution which is gradually filled by heating

around at a fixed location in $x$ while the shock moves upstream. It is only in the shock frame that they have become displaced downstream of the shock.

Considerable progress has been achieved recently in understanding the electron shock surfing process through high resolution one-dimensional fully relativistic full particle PIC simulations (Amano and Hoshino 2007) of a quasi-perpendicular $\left(\theta_{B n}=\right.$ $\left.80^{\circ}\right)$ high Mach number shock $\left(\mathscr{M}_{A}=15\right.$, Alfvén velocity $\left.V_{A}=0.05 c\right)$ and for an ion-to-electron mass ratio of $m_{i} / m_{e}=100$, upstream frequency ratio $\omega_{p e} / \omega_{c e}=$ 20 fixing the plasma density for a given upstream magnetic field, and plasma beta $\beta_{i}=\beta_{e}=0.08$. Note that for resolution of accelerated electrons a simulation should have to be relativistic. The mass ratio is still unsatisfactory (Matsukiyo and Scholer 2006b), as the Buneman instability ceases to be important at realistic high mass ratios $m_{i} / m_{e}=1,836$ when it is replaced by the MTSI. In addition, one-dimensionality of the simulation misses obliquely propagating waves and electrostatic structures and can thus be only approximate. It also misses the possibility of electrons becoming trapped in shock-rooted magnetic loops. Nevertheless, these simulations confirm shock surfing as a viable mechanism of electron acceleration and reveal a number of additional properties of shock surfing.

The electron energy distribution functions in these four regions are shown in Fig. 51. The interesting property of these distributions is that the field aligned surfing beam seen most pronounced at the foot edge does survive through all the four upstream regions. It is forming a bump on the distribution function, while the bulk distribution is heated ever more with approaching the shock. The height and width of this bump varies slightly, but the beam electrons are still identifiable until close to the shock, and neither their energy nor their intensity varies strongly. 
This suggests a very fast acceleration mechanism. Moreover, the bumps occur only on the upstream distribution and are thus identified as shock reflected electrons. Since electrons of such energies have not been present initially in the original inflowing electron distribution, these bumps and the corresponding electrons cannot be the result of mirror reflection by the Sonnerup-Wu mechanism until the distribution has become heated as there have not been any particles outside the loss cone. For the Sonnerup-Wu mirror-reflection mechanism the heating has to come first, and then the electrons can be reflected and accelerated in the upstream motional electric field. This acceleration is in the direction perpendicular to the magnetic field, however, while the electrons leave along the magnetic field. Note, however, that close to the shock transition the surfing electrons are heated almost isotropically.

Heating of the bulk distribution is clearly seen in Fig. 51. The distribution widens and, between the beam electrons and the bulk maximum, evolves into a region that exhibits an approximate power law shape with variable power. The final shape in this simulation is found to have power $F_{e}\left(\gamma_{e}\right) \propto \gamma^{-3.6}$. However, it is clearly the surfing beam electrons that possess high energy of several $10^{2}\left(\gamma_{1}-1\right) m_{e} c^{2}$. For instance, in the solar wind with its bulk energy of $\mathscr{E}_{e} \sim\left(\gamma_{1}-1\right) m_{e} c^{2} \sim 100 \mathrm{eV}$, this corresponds to an energy $\mathscr{E}_{e} \sim$ of several $10 \mathrm{keV}$, presumably sufficient for entering into the Fermi cycle for electrons. This can be concluded from a comparison of scales. For the Fermi mechanism the shock must be an infinitely thin surface. Electrons should thus have gyroradii $r_{c e} \gtrsim \Delta_{s}$. Since the shock width is of the order of $\Delta_{s} \sim 10^{3} \mathrm{~km}$, this implies electron energies of $\mathscr{E}_{e} \gtrsim 50 \mathrm{keV}$ in a $B_{1} \approx 5 \mathrm{nT}$ magnetic field. In addition sufficient scattering of these electrons off upstream and downstream turbulence is required for further acceleration.

Further insight into the surfing mechanism is obtained by following a selected surfing electron path. This is done in Fig. 52. The left panel shows the particle trajectory superimposed on the stacked magnetic field instead of the wave electric field. This has the advantage of directly seeing the motion of the shock ramp and formation of shock foot during quasi-perpendicular shock reformation. The physics, however, is similar to what was concluded by Hoshino and Shimada (2002). The particle moves into the shock at the upstream convective flow velocity, indicated by the straight line at the bottom. When encountering the shock foot edge, where the dense group of reflected gyrating ions accumulates, the inflow motion of the electron is suddenly truncated, and the electron starts oscillating in $x$ for $\sim 100 \omega_{c e}^{-1}$ electron cyclotron periods around an almost stationary position. During this time the shock ramp approaches the electron at the speed at which the shock jumps ahead quasi-periodically during quasi-perpendicular shock reformation while the electron remains in the foot region not being able to cross the shock ramp. On the contrary, it surfs along the shock ramp and is taken over by the next cycle of reflected ions, until the end of the simulation, turning together with the shock reflected foot ions into the upstream direction away from the ramp in the formation of the next reformation cycle.

We know of course that the electron is not at rest at position $x$. In fact, the electron moves in the $-y$-direction along the shock, being subject to acceleration by the upstream motional electric field. The excursions in its path in $x$ seen are the projections of the gyrations of the particle into the $(x, t)$-plane. Note that the injected electron had such a low temperature that the gyrations remained hidden in the convective straight 


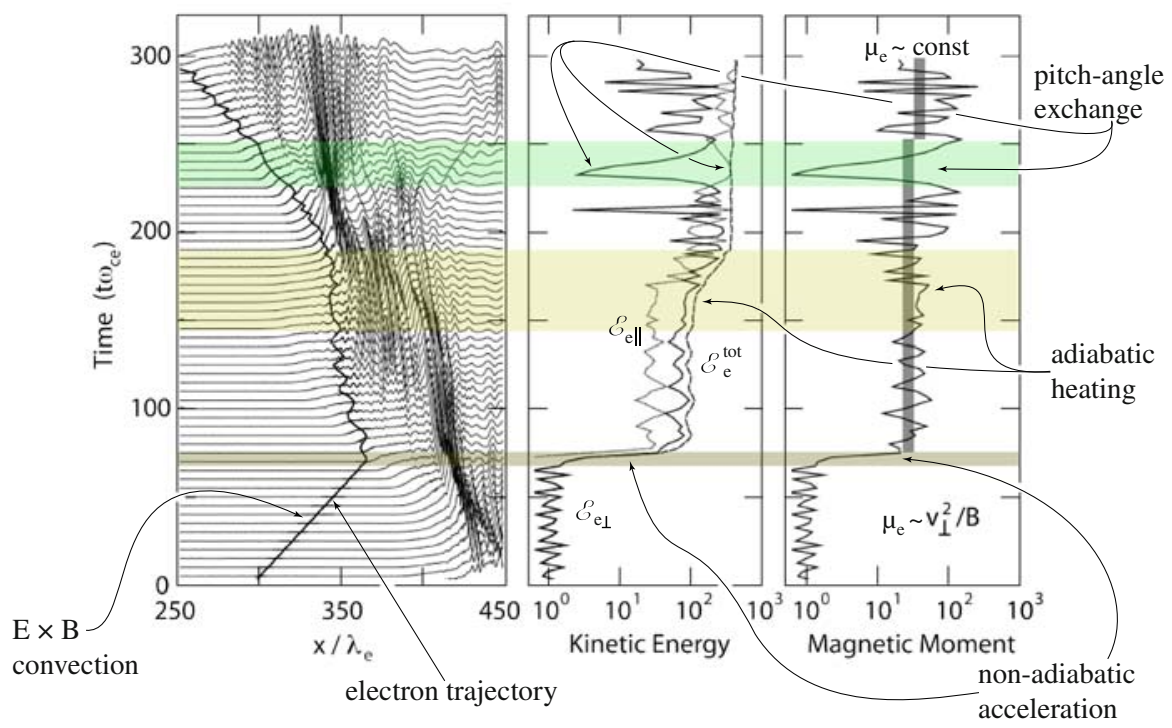

Fig. 52 Quasi-perpendicular shock electron acceleration by shock surfing (after Amano and Hoshino 2007) Left: The time-stacked evolution of the magnetic field in the shock simulation plotted after $t \omega_{c e}=$ $300\left(t \omega_{p e}=6,000\right)$. Two cycles of quasi-perpendicular shock reformation (with period $\sim 2 \omega_{c i}^{-1}$ ) are seen with the shock ramp jumping forward (to the left) against the upstream flow. The surfing electron trajectory (solid line) runs along the outer boundary of the shock foot. The electron is practically enslaved by the group of gyrating foot ions which are in excess of the flow and need to be charge neutralised. Center: Evolution of the electron energy in perpendicular and parallel components and also the total electron energy. The strongest acceleration happens in the first encounter of the electron with the foot ion group. It experiences further acceleration each time the reformation cycle ends. Note the stronger perpendicular acceleration of the electron causing an electron anisotropy $T_{e \perp}>T_{e \|}$. Right: The same for the electron magnetic moment $\mu_{e}$ showing that the initial energy gain within a time interval of $\Delta t \omega_{c e} \sim 5$ is highly non-adiabatic with the magnetic moment changing drastically. Afterwards the average magnetic moment (grey bar) is constant. Note also the phase of adiabatic heating in the increasing magnetic field which is mainly in the perpendicular energy

line electron path. The gyration becomes visible now because the electron has been violently accelerated at the encounter with the edge of the shock foot.

This acceleration is seen from the central panel, where the kinetic energy $\mathscr{E}_{e}=$ $\left(\gamma_{e}-1\right) m_{e} c^{2}$ of the electron has been plotted as a function of the simulation time. At the instant of the electron impact on the shock-foot edge, the electron energy suddenly increases by a factor $\sim 10^{2}$. This acceleration is non-adiabatic, which is indicated by the sudden change of the magnetic moment of the electron in the outer right panel at this time.

The non-conservation of the first electron adiabatic invariant $\mu_{e}=\mathscr{E}_{e \perp} / B$ signals that the interaction takes place on a scale that is shorter than the electron gyro-radius. The interaction is thus effectively non-magnetic. This can happen only when either the magnetic gradient is extraordinarily steep, or when the interaction becomes electrostatic on a scale shorter than the electron gyro-radius. As is seen from the left outer panel, the gradient is not steep enough for demagnetisation of the electron. Hence, the second case is realised here, and the interaction can be attributed to large amplitude solitary BGK modes which are excited at the edge of the shock foot. There they form 
small-scale localised electric potentials which act accelerating on the electrons. This process is the same as in the simulations by Hoshino and Shimada (2002).

From the central panel in Fig. 52 one finds that the acceleration is predominantly in the perpendicular direction, which is due to the trapping of the electron in the BGK mode at the location, where it comes to rest and experiences the motional electric field for a time of roughly $\sim 5 \omega_{c e}^{-1}$ gyroperiods. After this time the magnetic moment does not change anymore until the next reformation cycle starts at time $t \omega_{c e} \sim 250$.

The further increase in energy the electron experiences between $140<t \omega_{c e}<190$ is purely adiabatic, caused by the electron moving up into the stronger ramp magnetic field that acts as a mirror and reflects the electron by the mirror force-as proposed in the Sonnerup-Wu mechanism. However, since thermal upstream electrons cannot be reflected by no means in the Sonnerup-Wu mechanism, one realises that the preacceleration of the electron by the upstream convection electric field (which is made possible by trapping electrons in the electrostatic BGK wave field) is a necessary condition for shock reflection of electrons and their further acceleration.

In the time between the two acceleration phases, the non-adiabatic and the adiabatic mirror reflection, some reversible variations can be recognised in the magnetic moment. They do not change the particle energy. These variations are accompanied by reversible exchanges between the parallel and perpendicular energies of the electron. Variations of this kind are induced by changes in the direction of the magnetic field (that are probably caused by whistler waves attached to the shock ramp) and related pitch angle variations which do not interest us in the context of electron acceleration.

Applied to the solar wind, the electron energy gained in the non-adiabatic phase is roughly $\sim 10 \mathrm{keV}$, corresponding to a $\Delta \phi \sim 10 \mathrm{kV}$ electric potential drop. In a $B_{1} \sim 5 \mathrm{nT}$ field this yields a large acceleration rate of $\mathrm{d} \mathscr{E}_{e} / \mathrm{d} t \sim 1.5 \mathrm{MeV} / \mathrm{s}$. If the acceleration is due to the motional solar wind-electric field of $E_{\mathrm{sw}} \sim 2.5 \mathrm{mV} / \mathrm{m}$, the acceleration length is of the order of $\ell_{\text {acc }} \sim 4 \times 10^{3} \mathrm{~km}$ along the shock. Over this distance the electron remains to be in close contact with the BGK solitary wave. After leaving the BGK mode the accelerated electron has a large pitch angle against the magnetic field. It can now enter the Sonnerup-Wu mechanism for reflection at the shock, since it will be found outside the loss cone which, for a shock compression ratio of $B_{2} / B_{1} \sim 3$, is $\alpha_{\mathrm{lc}} \approx 35^{\circ}$.

\subsubsection{Quasi-parallel shock surfing}

From the above discussion of theory and simulation of electron surfing and acceleration at quasi-perpendicular shocks we learn that it is the combined effect of the interaction between the reflected ion and incoming electron components, the generation of localised electrostatic solitary structures of the BGK mode family, electron heating and acceleration in the motional electric field, and the shock mirror effect that are responsible for the generation of high energy electrons at quasi-perpendicular shocks. Even though this combination is very complicated, it works only for quasi-perpendicular shocks, and it works better the closer the shock is to being perpendicular, as then the number of reflected ions at a given supercritical Mach number is highest and the mirror effect on the electrons is largest. 
This raises the question as to whether or not quasi-parallel shocks could as well reflect and/or accelerate electrons. The question is difficult to answer. It has, however, been realised from hybrid and full particle simulations that quasi-parallel shocks reform due to the interaction of large amplitude magnetic pulsation. These pulsations give rise to the excitation of high frequency electrostatic waves and broadband electric noise, which can be understood only as the signature of many solitary like structures of the BGK-type family. Observations by CLUSTER have demonstrated the presence of such waves (see Sect. 4 on quasi-parallel shocks). Structures of this kind, as we know, are generated when the electron dynamics is taken into account. Quasi-parallel shocks therefore seem to host a highly active electron dynamics.

Moreover, during the reformation of quasi-parallel shocks the direction of the shock normal angle fluctuates considerably, identifying the quasi-parallel shock in many cases as a wave that locally is quasi-perpendicular on scales of the order of the tangential extension of the large amplitude pulsations or SLAMS. Locally, quasi-parallel shocks behave like quasi-perpendicular shocks. Ion reflection and foot formation happen to be present along the front of a pulsation when the pulsation (SLAMS) approaches the shock, though on a smaller scale than in the extended flat quasi-perpendicular case. Electrons do in this case surf along the pulsation and experience acceleration in the pulsation foot.

In a shock that is bent on a large global scale, the whole effect is extended over a much larger spatial region than the scale of the small genuinely quasi-perpendicular speck on the shock surface, where it is restricted solely to the shock foot region. In the quasi-parallel shock case, the entire region of presence of large amplitude pulsations in front of and at the ramp of the quasi-parallel foreshock will thus act as a region of electron surfing. Thus, at quasi-parallel shocks, electron reflection and acceleration is mediated by pulsations over the entire shock surface. So far PIC simulations could not prove this conjecture because of the difficulties involved in simulating three-dimensional quasi-parallel shocks with realistic mass-ratio. The related problems are open for future investigation.

\section{Conclusions}

There are two classes of collisionless shocks, a small class of subcritical shocks and a broad class of supercritical shocks.

We have argued that subcritical shocks will, in most cases, barely be observable in astrophysical systems. Their radiative signatures will most likely be immersed in the radiation caused by the general turbulent state of the medium. This may, for instance, be the case in clusters of galaxies where the magnetosonic velocity in the virialised intracluster gas is approximately of the same order of magnitude as the average velocity of the individual cluster galaxies. If this can be taken as a realistic picture of a cluster, only the fastest galaxies will exceed this velocity and possess extended bow shocks and wakes, but their bow shocks will probably be subcritical. The lack of observation of strong shock waves in clusters of galaxies might be partially due to this fact.

However, generally, all observed nonrelativistic astrophysical collisionless shocks belong to the second class of supercritical shocks. Their properties match-at least 
to some extent - the properties of collisionless supercritical shocks that have been described in the present review. Unfortunately, the extent to which they match these properties is uncertain. So far even the range of nonrelativistic Mach numbers has not been exhausted by simulations. This is due to computational reasons only. Mach numbers up to $\mathscr{M} \sim 30$ have occasionally been simulated, but it is not known whether shocks of much higher Mach number behave the same way. Nevertheless, in the nonrelativistic regime we are on comparably safe ground when applying the results of simulations to astrophysics. ${ }^{36}$

\subsection{Stellar wind termination shocks}

The first application we have in mind is to the properties of stellar wind termination shocks ${ }^{37}$ of which we have one accessible shock at hand, the heliospheric termination shock (for a recent review cf. Balogh et al. 2009). It is generated by the necessity of braking the solar wind when it interacts with the interstellar gas. In its course, the heliopause is formed as the outer boundary of the solar 'asterosphere', the heliosphere, at a radial distance of $R_{\mathrm{HP}} \sim 130 \mathrm{AU} \sim 1.3 \times 10^{6} \mathrm{R}_{\odot}$, the location, where the solar wind ram pressure

$$
\mathscr{E}_{\mathrm{SW}}(r)=\frac{1}{2} m_{i} V_{1}^{2} N(r) \approx 10^{-9}\left(\frac{r}{1 \mathrm{AU}}\right)^{2} \mathrm{~J} \mathrm{~m}^{-3}
$$

is balanced by the pressure of the interstellar medium

$$
P_{\mathrm{ISM}} \approx 2 \times 10^{-13} \mathrm{~J} \mathrm{~m}^{-3}
$$

The contribution of the heliospheric magnetic field can be neglected, because the solar wind Parker-spiral magnetic field decays with radial distance like $B \propto$ $B_{r=1}(r / 1 \mathrm{AU})^{-1}$. It is also believed that the interstellar magnetic field outside the heliosphere is not strong enough to substantially affect the pressure balance. ${ }^{38}$

These relations should also hold in other stellar-wind systems, which the interstellar pressure and solar wind energy being replaced by the local interstellar-gas pressure and the stellar-wind kinetic energy, and the reference distance of $1 \mathrm{AU} \approx 100 \mathrm{R}_{\odot}$ is scaled to the stellar reference distance. The location of the termination shock is the stand-off distance of the bow shock at a blunt concave obstacle. In this case the obstacle is the concave heliopause (asteropause). It yields a concave inward moving (in the solar or stellar wind frame) shock wave that is separated from the obstacle by the distance

\footnotetext{
36 We do not refer to relativistic shock simulations here, as this is another subtle problem of computational simulations. Numerical simulations are severely limited to low macro-particle numbers, small simulation boxes and short simulation times. Much of the results obtained by them are probably highly overestimated and exaggerated in their applicability to the astrophysical reality.

37 An early account, based on hydrodynamic calculations, was given by Bisnovatyi-Kogan and Silich (1995) in their Sections III.F and IV.E.

38 Magnetic-fields contribute to both the asymmetry and dynamics of the heliospheric boundary, however. Their role in reconnection, plasma transport, heating, and radiation is unknown. In addition, they are crucial in the dynamics of the termination shock.
} 


$$
\Delta_{\mathrm{TS}} / R_{\mathrm{HP}} \approx 1.1\left(N_{1} / N_{2}\right)
$$

Here $N_{1}$ is the solar (stellar) wind density inside the heliosphere (asterosphere), and $\mathrm{N}_{2}$ is the plasma density outside the termination shock in the turbulent heliosheath (asterosheath) ${ }^{39}$ The distance of the termination shock from the heliopause (asteropause) is thus inversely proportional to the shock compression ratio $\rho_{\mathrm{sh}}=N_{2} / N_{1}$. In the heliosphere this distance amounts to $R_{\mathrm{TS}} \approx 94 \mathrm{AU}$ which, incidentally, is just the distance where Voyager 1 crossed the termination shock. Under similar conditions the stand-off distance $R_{\text {STS }}$ of a stellar termination shock will be found at

$$
R_{\mathrm{STS}}=R_{\mathrm{AP}}\left[1-1.1 \rho_{\mathrm{STS}}^{-1}\right]
$$

expressed in terms of the astropause distance $R_{\mathrm{AP}}$ and the expected compression ratio $\rho_{\text {STS }}$ of the stellar termination shock. The astropause distance is determined from the dynamic pressure balance between the stellar wind ram pressure $\mathscr{E}_{\mathrm{STW}}\left(R_{\mathrm{AP}}\right)=$ $\frac{1}{2} m_{i} V_{\mathrm{STW}}^{2} N_{\mathrm{STW}}\left(r_{\mathrm{AP}}\right)$ and the about constant pressure $P_{\mathrm{ISM}}$ of the interstellar medium. In terms of the radius $R$ of the star it becomes

$$
R_{\mathrm{AP}}=R\left[\mathscr{E}_{\mathrm{STW}}(R) / P_{\mathrm{ISM}}\right]^{\frac{1}{2}}
$$

In a stellar wind of solar wind density but relative velocity $V \gtrsim 10 V_{\mathrm{SW}}$ with respect to the interstellar medium, the stand-off distance of the stellar-wind termination shock would be of the order of $R_{\mathrm{STS}} \sim 10^{3}\left(R / \mathrm{R}_{\odot}\right)$ AU, if it is assumed that the interstellar medium has the same pressure as the Local Interstellar Matter, and the shock-compression ratio has the canonical value $\rho_{\text {STS }} \sim 2$. Outflow velocities of "normal hot" (O type) stars (Kudritzki and Puls 2000) as function of effective temperature vary between $500 \lesssim V_{\mathrm{OS}} \lesssim 3,000 \mathrm{~km} / \mathrm{s}$, yielding $V \lesssim 6 V_{\mathrm{SW}}$, while the outflow velocity in Wolf-Rayet stars or other hot young stars may considerably exceed this number, reaching values of $V_{\mathrm{WRS}} \sim\left(10^{2}-10^{3}\right) V_{\mathrm{SW}}$.

Figure 53 shows the magnetic field trace of the farther inside (at $\sim 84 \mathrm{AU}$ ) heliospheric termination shock passage by Voyager 2. The heliospheric termination shock is identified as a highly supercritical shock possessing an extended foot/foreshock region and a surprisingly flat shock ramp. Also surprisingly is that the observed compression ratio is only $\rho_{\mathrm{sh}} \sim 2$, which suggests that the termination shock is not a very strong shock. Rather it is a moderately strong shock, in grave contrast to what had been expected. The shaded part in the figure is the ramp/overshoot transition. It has a rather gradual slope exhibiting a number of large-amplitude magnetic oscillations. Here the intense broadband noise was detected that is typical for shocks.

Similar conditions may be expected at stellar wind asterospheric termination shocks. The relative weakness of the shock is due to mass loading of the stellar wind with interstellar pick-up particles and, possibly, although to a much lesser degree, to the mediation by the cosmic-ray particles that have been accelerated by the shock (see Fig. 54). The presence of the broadband noise and the reflected particles in the

\footnotetext{
39 Seen from the interstellar gas, the termination shock can also be considered to be the bow shock of the interstellar gas in the solar (stellar) wind.
} 


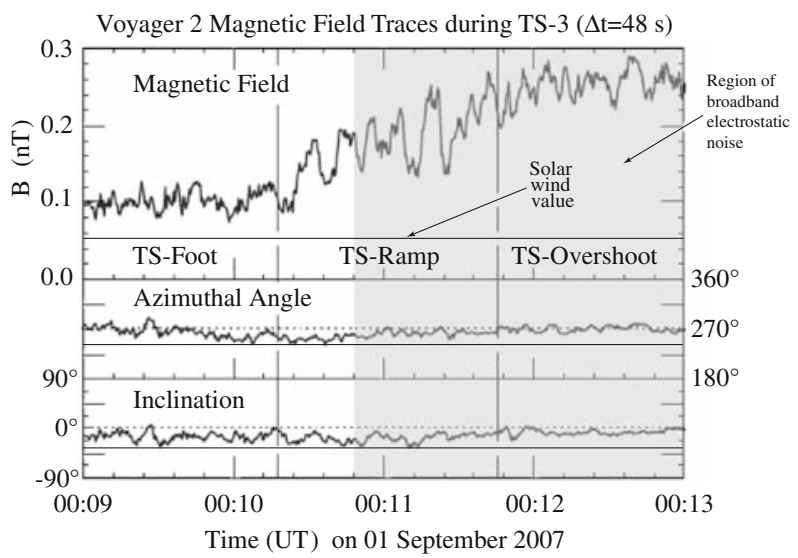

Fig. 53 The Voyager 2 termination shock (TS) crossing in the magnetic field trace (after Burlaga et al. 2008). The foot magnetic field is about twice the solar wind field. The ramp exhibits large amplitude coherent oscillations that are probably caused by ion trapping (field maxima coinciding with the vertexes of ion phase space rings). Incoherent fluctuations occur in the transition to the overshoot. Broadband electrostatic noise (Gurnett and Kurth 2008) is observed in the shaded region

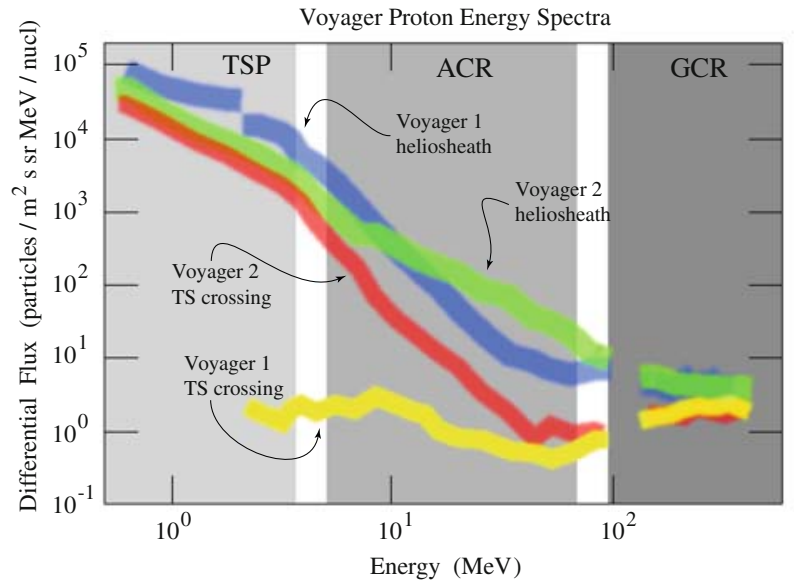

Fig. 54 Voyager high energy proton differential energy fluxes per nucleon (in the case of the protons this is per particle) as function of energy at heliospheric termination shock (TS) crossing and in the heliosheath (data taken from Stone et al. 2008). The width of the lines corresponds about to the error of the measurement. The energy range is divided into the three different populations: Termination Shock protons (TSP) $<(3-5)$ $\mathrm{MeV}$, Anomalous Cosmic Rays (ACR) (6-60) MeV, Galactic Cosmic Rays (GCR) > $90 \mathrm{MeV}$

foot region will cause the shock to radiate in radio frequencies. Larger stellar shocks caused by much stronger and faster outflows in stellar winds should accelerate much larger numbers of particles. If the number density in the accelerated particles and the astrospheric anomalous cosmic ray (ACR) component become large, such astrospheric termination shocks might emit visible radiation from radio to free-free radiation in the UV or soft X-rays.

The observed particle spectra which are reproduced in Fig. 54 and their possible origin have been discussed in detail elsewhere (see Balogh et al. 2009, Chap. 10). 
While shock acceleration seems to be responsible to push the spectra up in energy into the ACR range, the main acceleration of particles obviously takes place in the heliosheath proper and is not located at the termination shock. Apparently, the termination shock serves as the source of particle injection, while the main acceleration is due to trapping of energetic pre-accelerated shock particles in the compressed 'magnetic bottle' configuration of the Parker-spiral magnetic field in the heliosheath. These trapped particles are accelerated in a combination of their bounce motion inside the bottles with turbulent acceleration in the turbulent heliosheath plasma. Extrapolating these findings to stellar asterospheric termination shocks provides important insight into shock acceleration of particles in the extended asterospheres.

\subsection{Intra-stellar wind shocks}

Solar experience tells that all active stars eject matter into their stellar environment in the form of winds, flares and CMEs. Since active stars are magnetised, one may expect that the behaviour of this ejection of matter (plasma) resembles those forms observed in the solar environment.

Similar to the solar wind, stellar winds probably obey a distinct magnetic sector structure because of the multipolar properties of the mother-star magnetic field. This sector structure implies the presence of a current sheet and a Parker-spiral general magnetic-field geometry. Production of the stellar wind remains to be an unresolved problem. Micro-shocks that may be generated at the bottom of the stellar coronae are among the candidate mechanisms for accelerating the winds. Such shocks are presumably subcritical and thus dissipative, however.

The interesting class of shocks in the stellar wind are flare shocks and mass ejection shocks, the former produced by blast waves, the latter are piston driven shocks. Both corotate with the star and thus cause corotating interaction shocks, forward and reverse shocks of similar kind as observed in the heliosphere. Forward shocks are mostly quasi-perpendicular, reverse shocks are of high Mach number and because of their inclination tend to be magnetically quasi-parallel. These shocks are known to accelerate particles to high, though mildly relativistic energies.

Typical Mach numbers in the solar wind range around $2<\mathscr{M}<12$. In stellar winds the Mach numbers depend on the stellar wind conditions. In very fast winds, forward shocks can be low Mach number, while reverse shocks may reach very high Mach numbers, thus being of primary importance in the production of radiation and the acceleration of charged particles. The best candidates for such shocks are variable stars, flare stars and young hot stars with their strong wind outflows. Time dependences of the outflow velocities from ordinary stars (Kudritzki and Puls 2000) indicate the high temporal variability of stellar wind flows also in these objects. Under such conditions, shocks are expected to evolve in the outer asterospheres (Hillier et al. 1993, 1998; Owocki and Puls 1999). Here, they result from interacting wind streams of different speeds which may overtake each other and cause internal shocks. Generation of such shocks is strongly affected by the corotation of the stellar wind magnetic field out to radial stellar distances where the corotation is not destroyed by the centrifugal forces 


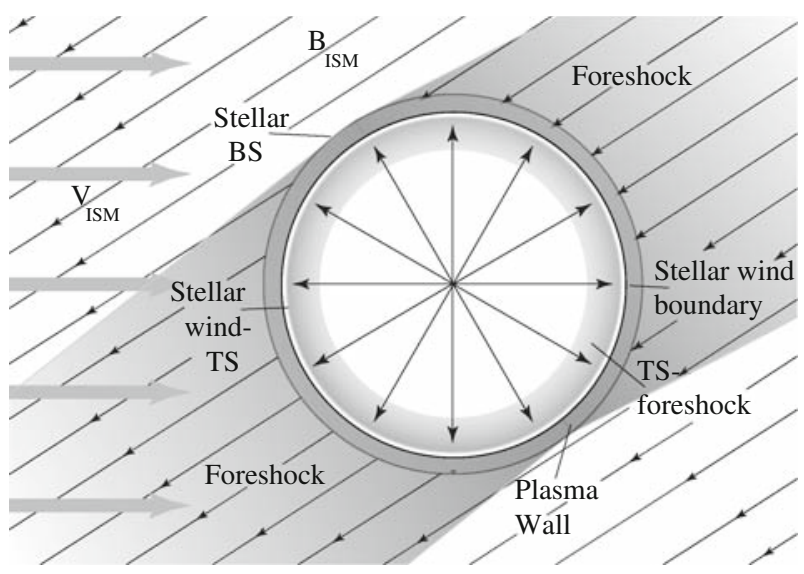

Fig. 55 Sketch of a stellar shock system generated in analogy to the heliospheric shock system in the interaction of the stellar wind with the interstellar medium (velocity $V_{\text {ISM }}$, magnetic field $B_{\text {ISM }}$ ). It consists of a bow shock, plasma wall and termination shock. Foreshock wings of the BS have provisionally been included. Depending on the geometry of the (possibly Parker spiral) stellar wind magnetic field the latter may possess a foreshock which also contains interstellar pick-up ions

that act on the frozen-in plasma. In similarity to CMEs, the shocks may sometimes also result from the passage of stellar ejecta across the outflowing stellar wind.

Interaction of the fast stellar winds with the surrounding interstellar medium will, in addition to causing the asterospheric termination shock, generate asterospheric bow shocks in the interstellar medium. Such bow shocks are the consequence of the super-magnetosonic velocity difference between the asterosphere and the interstellar medium, which is in complete similarity to the heliosphere. The heliosphere, playing the role of a compact obstacle in the interstellar flow, is believed to be responsible for the production of a heliospheric bow shock in the Local Interstellar Medium (cf. Fig. 55).

\subsection{Supernova remnant shocks}

SNR shocks are favoured as the main agent in the acceleration of high-energy cosmic ray particles (cf., e.g. Blandford and Eichler 1987). Presumably, they are indeed most important in producing energetic cosmic rays, in particular in the process of injection and distribution of the heavy elements that have been produced in the Supernova explosions. When dealing with nonrelativistic shocks in SNRs, we are probably dealing with a small subclass of SNR shocks only, because it is assumed that most of them are at least mildly relativistic if not relativistic, even those shocks that are believed to exist in the outermost shells of the SNR. Nevertheless, shocks in the region of contact with the interstellar matter may be non-relativistic.

To get an impression on the initial conditions, we note that the interstellar matter densities are of the order of $N_{\text {ISM }} \lesssim 10^{5} \mathrm{~m}^{-3}$, while the outer shell expansion speeds of the remnant are in the velocity interval $1,500<V<3,000 \mathrm{~km} / \mathrm{s} \lesssim 0.01 c$. Temperatures of the ionised component are around $T_{i} \sim 1 \mathrm{eV}$ for protons, corresponding to 


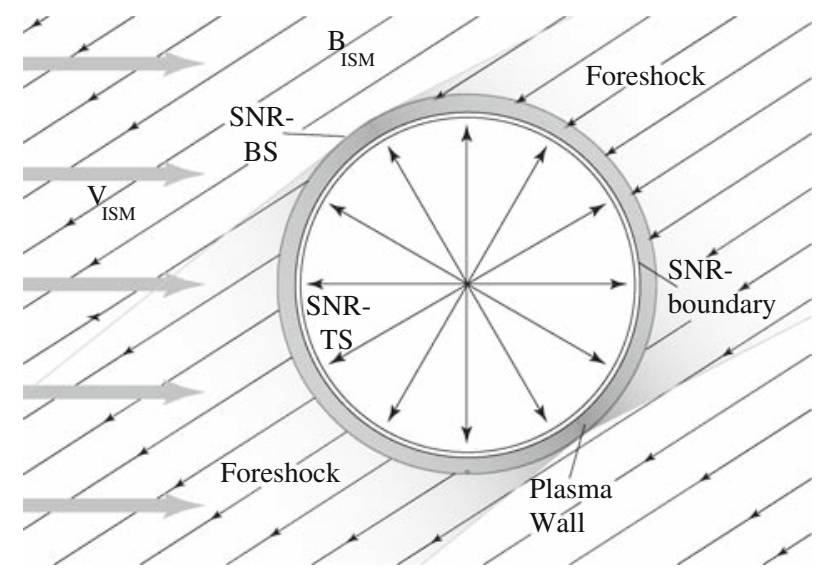

Fig. 56 A sketch of a possible supernova remnant shock system in the interstellar medium (velocity $V_{\text {ISM }}$ and magnetic field $B_{\text {ISM }}$ ) which, similar to stellar termination shocks, consists of a bow shock, plasma wall (generated by the snow-plough effect of the fast expanding SNR) and SNR terminations shock. The latter probably lacks an inner foreshock. The foreshock wings of the BS are narrow and do not extend far upstream because of the fast expansion of the SNR. the wings are populated only by high energy particles that may have been accelerated by the SNR shock system

thermal speeds $v_{i} \sim 10 \mathrm{~km} / \mathrm{s}$. Magnetic fields are of the order of $B \sim 0.5 \mathrm{nT}$ yielding $V_{A} \sim(5-10) \mathrm{km} / \mathrm{s}$ or a magnetosonic speed of $c_{m s} \sim 10 \mathrm{~km} / \mathrm{s} .{ }^{40}$ This suggests a Mach number of $80<\mathscr{M}<200$ which is large but still within our allowed parameter range. A reasonable estimate of the electron temperature in the outermost remnant shells is of the order of $1 \lesssim T_{e} \lesssim 10 \mathrm{keV} \sim 0.02 m_{e} c^{2}$. At Mach numbers this high but still moderate (which so far have not yet been accessible to numerical shock simulation), theory predicts that the ion-Weibel instability assumes a large enough growth rate to generate magnetic field barriers, which increase the reflection capability of the shock while, at the same time, causing localised structures in the shock transition.

Under such conditions non-relativistic collisionless shock theory may apply. Therefore, we may conclude that the approximately circular shape of the outer bow shock of the SNR identifies this shock as a non-relativistic collisionless, supercritical, mostly quasi-parallel and high Mach number shock. It propagates in the (approximately) straight interstellar magnetic field. Such a bow shock possesses a extended foreshocks. In the small areas in front of its quasi-perpendicular parts, it also possesses shock-foot regions, a shock transition and a broad turbulent downstream region which separates the shock from what comes behind, forming the SNR obstacle. However, since the SNR expands at very high speed, the tilt angle of the foreshock is small, and the reflected ions have no time to escape far before being caught again by the expanding supernova shell. This causes low populated foreshock wings on the supernova bow shock (a sketch is given in Fig. 56). This fast expansion and re-trapping, particularly in the quasi-parallel parts of the supernova bow shock, should indeed cause multiple reflection and therefore strong acceleration of particles.

\footnotetext{
40 One should, however, note that these values are rather uncertain. It is not excluded that even the outermost shocks in the SNR are all relativistic, in which case the theory developed in this review can serve only as a rough guide line.
} 
The shock ramp/transition of this bow shock is not wider than a few ion inertial lengths. With the parameters given, this is not more than at most $\Delta_{\text {sh }} \lesssim$ few $\left(10^{3}-10^{4}\right)$ $\mathrm{km}$, far below any observational resolution.

Behind the bow shock comes a ring of high density gas/plasma, the plasma wall which is a result of the pile-up of plasma due to the snow-plough action of the expanding SNR. This region is bounded from the inside by a discontinuity, the nature of which is not clear in the magnetised case. It might be a tangential discontinuity, however, similar to the heliopause ${ }^{41}$ or, possibly, similar to the magnetopauses of magnetised planets. At this location, reconnection between the stellar wind and interstellar magnetic fields might also take place. Reconnection would connect the SNR and interstellar magnetic fields and generate particle beams and flows.

Inside of this plasma wall and discontinuity one will find the supernova termination shock. Its structure depends on the imprecisely known structure of the SNR magnetic field. This termination shock will not possess any interior foreshock region inside of the termination shock because, probably, the magnetic field in the fast SNR flow is completely azimuthal. This is in substantial contrast to stellar wind termination shocks.

The question arises, which of these structures are seen in the observations. According to the properties of supercritical quasi-parallel shocks, neither of the shocks exhibits a smooth spherical shape (as would be suggested by the sketch in Fig. 56). The bow shock, due to the quasi-parallel turbulent reformation process, breaks off into many small-scale substructures that are distributed along the spherical bow-shock surface. The high resolution optical pictures of outer SNR shocks show just such a broken shock structure that consists of many shock patches. Each of these patches is, however, still orders of magnitude larger than any of the transition scales that are inferred from theory and simulation when applied to the SNR parameters. The achievable resolution is still completely insufficient to resolve the shock on these fine scales.

From the heliospheric paradigm we also learned that the SNR not only generates its outer bow shock but snowploughs the interstellar material up into the dense plasmawall that surrounds the expanding remnant cloud. The diameter of this wall in the case of the heliosphere is a fraction of the diameter of the heliosphere, and the matter is of comparably high density. Hence, it lies in between the SNR termination shock and the bow shock which both accelerate large numbers of particles to high energies which are filling the space in between. Both SNR shocks are high Mach-number shocks and should be very efficient in the generation of high-energy particles. Indeed, collisionless shock-simulations suggest that these particles hang around the shock upstream as well as downstream, filling the large parts of the region between the shocks including the wall. After thermalisation, radiation may be emitted by the electrons from these regions that can be detected from Earth. Resolution of the puzzle of the nature of the observed SNR shocks is a very important field of research.

\footnotetext{
41 It should be noted that the nature of the heliopause is not known either. It could be a kind of tangential discontinuity, as is assumed in application of pure pressure balance. But since one also believes that plasma penetrates across the heliopause into both directions, it may rather be a more complex transition layer.
} 


\subsection{Galactic wind shocks?}

It is not only stars which possess outflows that bear the name of stellar winds. Occasionally, galaxies also blow strong winds into the intergalactic space. This happens if the ionised gas in the galaxy overcomes the galactic binding velocity.

These galactic winds are the primary mechanism by which energy and metals are recycled in the galaxy and deposited into the intergalactic medium, in particular into the intracluster gas (a recent review can be found in Veilleux et al. 2005). Some galactic winds are driven by stellar winds (if they do not reach equilibrium with the interstellar environment thereby forming expanding shells which may by themselves be intragalactic shocks). They are caused by supernovae, starbursts, and possibly also by intragalactic black holes. Galactic winds are also emitted from AGNs.

Terminal velocities of the winds are presumably of the order of $V_{\mathrm{GW}} \approx 3,000 \sqrt{\alpha / \Lambda}$ $\mathrm{km} / \mathrm{s}$, where $\Lambda$ is the mass loading factor, and $\alpha$ is the efficiency coefficient for thermalisation of the mechanical energy, i.e. the rate at which flow energy is transferred into heat. Clearly such winds escape easiest in the direction perpendicular to the galactic disk and are thus improbable in elliptical galaxies (cf., the review by Mathews and Brighenti 2003). They are seen in $\mathrm{H} \alpha$ and, if sufficiently hot, show also signatures in X-rays out to distances of $\sim 20 \mathrm{kpc}$. Such emissions are believed to come from electrons that have been accelerated in wind shocks.

From the detection of galactic radio emission it has been found that the winds are magnetised, containing fields up to $B \approx$ a few $0.1 \mathrm{nT}$. It is believed that these winds may indeed escape into the intergalactic medium from galaxies with rotation velocities $\lesssim 300 \mathrm{~km} / \mathrm{s}$. Active galaxies may, however, have much stronger winds and thus produce fast outflows. It is, however, not known whether galactic termination shocks form in the intracluster medium. For this one would need to know the ram pressures in both media. Intracluster media have temperatures of a few keV but low densities, which are mainly the result of the contribution from the galactic winds. Densities in escaping galactic winds are barely known yet. Thus it remains to be unclear, whether or not galactic termination shocks can form at all. In any case they need the galaxy to be a member of a cluster, being embedded into the intergalactic medium.

\subsection{Summary of results}

This review has collected our current knowledge of the formation and properties of non-relativistic supercritical collisionless shocks. We may summarise the main points as follows:

1. Non-relativistic collisionless shocks have Mach numbers $\ll 10^{4}$. They are mostly supercritical. Subcritical shocks which might be numerous are 'dark' and thus invisible. Since supercritical shocks cannot digest the energy density of the inflow they reflect a substantial part of the upstream ions back upstream.

2. Quasi-perpendicular shocks form a narrow foot upstream in front of the shock ramp where the reflected ions are accelerated into a current that flows tangentially 
to the shock and which together with the reflected ions serves as reformator of the shock.

3. Quasi-parallel shocks reflect particles upstream in a turbulent reflection process which is a combination of the reformation of the shock from upstream turbulence and particle energisation.

4. Reformation in quasi-parallel shocks is due to the arrival of large amplitude upstream pulsations. It makes the shock highly variable in time and, in addition, inhomogeneous and structured in a tangential direction along the shock surface.

5. Shock transitions have a width of the order of at most a few ion inertial lengths $\lambda_{i}=c / \omega_{p i}$. From an astrophysical point of view, non-relativistic collisionless shocks are thus microscopically narrow transitions which are not resolved by observations, unless they accelerate particles in sufficiently high number to large energies.

6. Due to the generation of upstream foreshock turbulence all quasi-parallel shocks are locally quasi-perpendicular. This property makes them very good particle reflectors which are capable of accelerating a fraction of particles to energies high enough for starting Fermi acceleration. The number of accelerated particles increases with Mach number.

7. One therefore expects that high Mach number astrophysical shocks reflect and pre-accelerate a substantial fraction of the background ions to high energies. The reflectivity might be increased by the excitation of the ion-Weibel instability in the shock foot and ramp which is favoured at high Mach numbers. It generates and adds magnetic fields to the shock ramp at the expense of the energy of the upstream flow.

8. Electrons are accelerated in a different way by a complicated shock surfing mechanism in which the microphysics of the shock plasma is involved.

9. These electrons are capable of radiating in radio waves by wave-wave interaction processes which produce shock radiation at the local plasma frequency and its harmonics. Under favourable conditions for electron hole (BGK-mode) formation in combination with strong magnetic fields evolving from the Weibel instability, the electron-cyclotron maser mechanism might locally cause direct excitation of free-space radio waves in the shock transition layer.

10. Radiation at higher energies (e.g. in X-rays) requires the thermalisation of the plasma and transfer of energy from the ions to electrons in a large volume in order to reach sufficiently high emissivities that it can be observed remotely. This kind of thermalisation can possibly be due to electron heating in the course of electron hole formation (BGK-modes) in the shock transition layer.

Acknowledgements The invitation to this review by the Editors, M.C.E. Huber and T. Courvoisier is deeply acknowledged, as are their continuous encouragements. I thank A. Balogh (ISSI), M. Hoshino and C. H. Jaroschek (both Tokyo University), S. Matsukiyo (Kyushu University), R. Pottelette (CETP St Maur, Paris), and M. Scholer (MPE) for information and discussion. Part of this review has been prepared during a Visiting Scientist period at ISSI Bern. The support of the librarians, Irmela Schweizer and Andrea Fischer, was crucial for its preparation. This research has also benefitted from a Gay-Lussac award of the French Government. 


\section{References}

Amano T, Hoshino M (2007) Electron injection at high Mach number quasi-perpendicular shocks: surfing and drift acceleration. Astrophys J 661:190-202

Bale SD, Mozer FS (2007) Measurement of large parallel and perpendicular electric fields on electron spatial scales in the terrestrial bow shock. Phys Rev Lett 98:205001. doi:10.1103/PhysRevLett.98. 205001

Bale SD et al (2002) Electrostatic turbulence and Debye-scale structures associated with electron thermalization at collisionless shocks. Astrophys J 575:L25-L28

Bale SD, Mozer FS, Horbury TS (2003) Density-transition scale at quasiperpendicular collisionless shocks. Phys Rev Lett 91:265004

Balikhin MA, Krasnoselskikh V, Gedalin MA (1995) The scales in quasiperpendicular shocks. Adv Space Res 15:247

Balikhin MA et al (2005) Ion sound wave packets at the quasiperpendicular shock front. Geophys Res Lett 32:L24106. doi:10.1029/2005GL024660

Balogh A, Riley P (2005) Overview of heliospheric shocks. In: Jokipii JR, Sonett CP, Giampapa MS (eds) Cosmic winds and the heliosphere. The University of Arizona Press, Tucson, pp 359-388

Balogh A, Klein KL, Treumann RA (2009) The physics of shock waves in collisionless space plasmas. ISSI SR-10, ESA-ESTEC, Noordwijk, The Netherlands, pp 1-500

Baumjohann W, Treumann RA (1996) Basic space plasma physics. Imperial College Press, London

Behlke R et al (2003) Multi-point electric field measurements of short large-amplitude magnetic structures (SLAMS) at the Earth's quasi-parallel bow shock. Geophys Res Lett 30:1177. doi:10.1029/ 2002GL015871

Behlke R et al (2004) Solitary structures associated with short large-amplitude magnetic structures (SLAMS) upstream of the Earth's quasiparallel bow shock. Geophys Res Lett 31:L16805. doi:10. 1029/2004GL019524

Biskamp D (1973) Collisionless shock waves in plasmas. Nucl Fusion 13:719-740

Biskamp D (2003) Magnetohydrodynamic turbulence. Cambridge University Press, Cambridge

Bisnovatyi-Kogan GS, Silich SA (1995) Shock-wave propagation in the nonuniform interstellar medium. Rev Mod Phys 67:661-712

Blandford R, Eichler D (1987) Particle acceleration at astrophysical shocks: a theory of cosmic ray origin. Phys Rep 154:1-75

Burgess D (1987) Shock drift acceleration at low energies. J Geophys Res 92:1119-1130

Burgess D (1995) Collisinless shocks. In: Kivelson MG, Russell CT (eds) Introduction to space physics. Cambridge University Press,, Cambridge pp 129-163

Burlaga LF et al (2008) Magnetic fields at the solar wind termination shock. Nature 454:75-77. doi:10. 1038/nature07029

Coroniti FV (1970) Dissipation discontinuities in hydromagnetic shock waves. J Plasma Phys 4:265

Décréau PME et al (2001) Early results from the Whisper instrument on cluster: an overview. Ann Geophysicae 19:1241-1258

De Hoffman F, Teller E (1950) Magneto-hydrodynamic shocks. Phys Rev 80:692-703

Dickel JR, Wang S (2004) Non-thermal X-ray and radio emission from the SNR N 157B. Adv Space Res 33: 446-449. doi:10.1016/j.asr.2003.08.023

Diehl R (ed) et al (2002) The astrophysics of galactic cosmic rays. Space Science Series of ISSI, vol 13. Springer, New York

Dubouloz N, Scholer M (1995) Two-dimensional simulations of magnetic pulsations upstream of the Earth's bow shock. J Geophys Res 100:9461-9474

Edmiston JP, Kennel CF (1984) A parametric survey of the first critical Mach number for a fast MHD shock. J Plasma Phys 32:429-441

Eselevich VG (1982) Shock-wave structure in collisionless plasmas from results of laboratory experiments. Space Sci Rev 32:65-81

Feldman WC et al (1983) Electron velocity distributions near the earth's bow shock. J Geophys Res 87: 96-110

Formisano V, Torbert R (1982) Ion acoustic wave forms generated by ion-ion streams at the earth's bow shock. Geophys Res Lett 9:207-210

Galeev AA, Krasnosel'skikh VV, Lobzin VV (1988) Fine structure of the front of a quasi-perpendicular supercritical collisionless shock wave. Sov J Plasma Phys 14:697-702 
Gedalin MA (1997) Ion heating in oblique low-Mach number shocks. Geophys Res Lett 24:2511-2514

Giacalone J (2004) Large-scale hybrid simulations of particle acceleration at a parallel shock. Astrophys J 609:452-458

Giacalone J (2005) The efficient acceleration of thermal protons by perpendicular shocks. Astrophys J 628:L37-L40

Giacalone J, Neugebauer M (2008) The energy spectrum of energetic particles downstream of turbulent collisionless shocks. Astrophys J 673:629-636

Ginzburg VL, Zheleznyakov VV (1958) On the possible mechanisms of sporadic solar radio emission (radiation in an isotropic plasma). Sov Astron 2:653

Goldstein ML, Roberts DA, Matthaeus WM (1995) Magnetohydrodynamic turbulence in the solar wind. Ann Rev Astron Astrophys 33:283-325

Goodrich CC, Scudder JD (1984) The adiabatic energy change of plasma electrons and the frame dependence of the cross-shock potential at collisionless magnetosonic shock waves. J Geophys Res 89: 6654-6662

Gurnett DA (1985) Plasma waves and instabilities. In: Tsurutani BT, Stone RG (eds) Collisionless shocks in the heliosphere: reviews of current research. Amer Geophys Union, Washington DC pp 207-224

Gurnett DA, Kurth WS (2008) Intense plasma waves at and near the solar wind termination shock. Nature 454: 78-80. doi:10.1038/nature07023

Gurnett DA, Neubauer FM, Schwenn R (1979) Plasma wave turbulence associated with an interplanetary shock. J Geophys Res 84:541-552

Hada T, Oonishi M, Lembège B, Savoini P (2003) Shock front nonstationarity of supercritical perpendicular shocks. J Geophys Res 108:1233

Hillier DJ et al (1993) The 0.1-2.5-KEV X-ray spectrum of the O4F-STAR Zeta-Puppis. Astron Astrophys 276:117

Hillier DJ et al (1998) An optical and near-IR spectroscopic study of the extreme P Cygni-type supergiant HDE 316285. Astron Astrophys 340:438

Hobara Y et al (2008) Cluster observations of electrostatic solitary waves near the Earth's bow shock. J Geophys Res 113:A05211. doi:10.1029/2007JA012789

Hoshino M (2001) Nonthermal particle acceleration in shock front region: "Shock Surfing Accelerations". Progr Theor Phys Suppl 143:149-181

Hoshino M, Shimada N (2002) Nonthermal electrons at high Mach number shocks: electron shock surfing acceleration. Astrophys J 572:880-887

Hull AJ et al (2006) Large-amplitude electrostatic waves associated with magnetic ramp substructure at Earth's bow shock. Geophys Res Lett 33:L15104. doi:10.1029/2005GL025564

Jaroschek CH, Lesch H, Treumann RA (2004) Self-consistent diffusive lifetimes of Weibel magnetic fields in gamma-ray bursts. Astrophys J 616:1065-1071

Jaroschek CH, Lesch H, Treumann RA (2005) Ultrarelativistic plasma shell collisions in $\gamma$-ray burst sources: dimensional effects on the final steady state magnetic field. Astrophys J 618:822-831

Jeffrey A, Taniuti T (1964) Nonlinear wave propagation. Academic Press, New York

Kantrowitz A, Petschek HE (1966) MHD characteristics and shock waves. In: Kunkel WB (ed) Plasma physics in theory and application. McGraw-Hill, New York, pp 148-207

Karpman VI (1964) Structure of shock front propagating at an angle to a magnetic field in a low-density plasma. Sov Phys-Tech Phys 8:715-719

Karpman VI, Sagdeev RZ (1964) The stability of a shock front moving across a magnetic field in a rarefied plasma. Sov Phys Tech Phys 8:606-611

Kennel CF, Sagdeev RZ (1967) Collisionless shock waves in high beta plasma 1 \& 2. J Geophys Res 72:3303-3326; 3327-3341

Kennel CF, Edmiston JP, Hada T (1985) A quarter century of collisionless shock research. In: Stone RG, Tsurutani BT (eds) Collisionless shocks in the heliosphere: a tutorial review. Amer Geophys Union, Washington DC, pp 1-36

Kis A et al (2004) Multi-spacecraft observations of diffuse ions upstream of Earth's bow shock. Geophys Res Lett 31:L20801. doi:10.1029/2004GL020759

Kis A et al (2007) Scattering of field-aligned beam ions upstream of Earth's bow shock. Ann Geophysicae 25:785-799

Krimigis SM, Zwickl RD, Baker DN (1985) Energetic ions upstream of Jupiter's bow shock. J Geophys Res 90(A5):3947-3960 
Kucharek H, Scholer M (1991) Origin of diffuse superthermal ions at quasi-parallel supercritical collisionless shocks. J Geophys Res 96:21195-21205

Kudritzki RP, Puls J (2000) Winds from hot stars. Ann Rev Astron Astrophys 38:613-666

Landau LD, Lifshitz EM (1959) Fluid mechanics. Addison-Wesley, Reading

Lax PD (1957) Hyperbolic systems of conservation laws II. Commun Pure Appl Math 10:537

Lee MA (1982) Coupled hydromagnetic wave excitation and ion acceleration upstream of the earth's bow shock. J Geophys Res 87:5063-5080

Lembège B, Savoini P (2002) Formation of reflected electron bursts by the nonstationarity and nonuniformity of a collisionless shock front. J Geophys Res 107(A3):1037

Lembège B et al (1999) The spatial sizes of electric and magnetic field gradients in a simulated shock. Adv Space Res 24:109-112

Liberman MA, Velikhovich L (1986) Physics of shock waves in gases and plasmas. Springer Verlag, Berlin

Lucek EA et al (2002) Cluster magnetic field observations at a quasi-parallel bow shock. Ann Geophysicae 20:1699-1710

Marshall W (1955) The structure of magneto-hydrodynamic shock waves. Proc R Soc Lond A 233:367

Mathews WG, Brighenti F (2003) Hot gas in and around elliptical galaxies. Ann Rev Astron Astrophys 41: 191-239. doi:10.1146/annurev.astro.41.090401.0945542

Matsukiyo S, Scholer M (2003) Modified two-stream instability in the foot of high Mach number quasiperpendicular shocks. J Geophys Res 108:1459

Matsukiyo S, Scholer M (2006a) On microinstabilities in the foot of high Mach number perpendicular shocks J Geophys Res 111:A06104

Matsukiyo S, Scholer M (2006b) On reformation of quasi-perpendicular collisionless shocks. Adv Space Res 38:57-63

Narita Y, Glassmeier K-H, Treumann RA (2006) Wave-number spectra and intermittency in the terrestrial foreshock region. Phys Rev Lett 97:191101

Narita Y, Glassmeier K-H, Gary SP, Goldstein ML, Treumann RA (2009) Analysis of wave number spectra through the terrestrial bow shock. J Geophys Res 114 (in press)

Ness NF et al (1979) Magnetic field studies at Jupiter by Voyager 1-preliminary results. Science 204: 982-986

Ohsawa Y (1985a) Strong ion acceleration by a collisionless magnetosonic shock wave propagating perpendicularly to a magnetic field. Phys Fluids 28:2130-2136

Ohsawa Y (1985b) Nonlinear magnetosonic fast and slow waves in finite beta plasmas and associated resonant ion acceleration. J Phys Soc Jpn 54:4073-4076

Oka M et al (2006) Whistler critical Mach number and electron acceleration at the bow shock: geotail observation. Geophys Res Lett 33:L24104

Owocki SP, Puls J (1999) Line-driven Stellar winds: the dynamical role of diffuse radiation gradients and limitations to the Sobolev approach. Astrophys J 510:355

Papadopoulos K (1988) Electron heating in superhigh Mach number shocks. Astrophys Space Sci 144: 535-547

Paschmann G et al (1981) Characteristics of reflected and diffuse ions upstream from the earth's bow shock. J Geophys Res 86:4355-4364

Pickett JS et al (2004) On the generation of solitary waves observed by Cluster in the near-Earth magnetosheath. Nonlinear Process Geophys 12:181-193

Sagdeev RZ (1966) Cooperative phenomena and shock waves in collisionless plasmas. Rev Plasma Phys 4:23-91. Leontovich MA (ed) Consultants Bureau, New York

Sagdeev RZ, Shapiro VD (1973) Influence of transverse magnetic field on Landau damping. JETP Lett (Engl Transl) 17:279-283

Schmitz H, Chapman SC, Dendy RO (2002) Electron preacceleration mechanisms in the foot region of high Alfvénic Mach number shocks. Astrophys J 570:637-646

Scholer M (1993) Upstream waves, shocklets, short large-amplitude magnetic structures and the cyclic behavior of oblique quasi-parallel collisionless shocks. J Geophys Res 98:47-57

Scholer M, Burgess D (2006) Transition scale at quasiperpendicular collisionless shocks: full particle electromagnetic simulations. Phys Plasmas 13:062101

Scholer M, Burgess D (2007) Whistler waves, core ion heating, and nonstationarity in oblique collisionless shocks. Phys Plasmas 14:072103

Scholer M, Kucharek H (1999a) Interaction of pickup ions with quasi-parallel shocks. Geophys Res Lett 26:29-32 
Scholer M, Kucharek H (1999b) Dissipation, ion injection, and acceleration in collisionless quasi-parallel shocks. Astrophys Space Sci 264:527-543

Scholer M, Matsukiyo S (2004) Nonstationarity of quasi-perpendicular shocks: a comparison of full particle simulations with different ion to electron mass ratio. Ann Geophysicae 22:2345-2353

Scholer M, Kucharek H, Giacalone J (2000) Cross-field diffusion of charged particles and the problem of ion injection and acceleration at quasi-perpendicular shocks . J Geophys Res 105:18285-18293

Scholer M, Shinohara I, Matsukiyo S (2003) Quasi-perpendicular shocks: length scale of the cross-shock potential, shock reformation, and implication for shock surfing. J Geophys Res 108:1014. doi:10. 1029/2002JA009515

Schwartz SJ, Thomsen MF, Gosling JT (1983) Ions upstream of the earth's bow shock-a theoretical comparison of alternative source populations. J Geophys Res 88:2039-2047

Sckopke N et al (1983) Evolution of ion distributions across the nearly perpendicular bow shock-specularly and non-specularly reflected-gyrating ions. J Geophys Res 88:6121-6136

Scudder JD (1995) A review of the physics of electron heating at collisionless shocks. Adv Space Res 15:181-223

Scudder JD et al (1986) The resolved layer of a collisionless, high beta, supercritical, quasi-perpendicular shock wave. J Geophys Res 91:11019-11097

Shimada N, Hoshino M (2000) Strong electron acceleration at high mach number shock waves: simulation study of electron dynamics. Astrophys J 543:L67-L71

Shimada N, Hoshino M (2005) Effect of strong thermalization on shock dynamical behavior. J Geophys Res 110:A02105. doi:10.1029/2004JA010596

Shlesinger MF, Zaslavsky GM, Klafter J (1993) Strange kinetics. Nature 363:31-37

Sonnerup BUÖ (1969) Acceleration of particles reflected at a shock front. J Geophys Res 74:1301-1304

Stone EC et al (2008) An asymmetric solar wind termination shock. Nature 454:71-74. doi:10.1038/ nature 07022

Sugiyama T, Terasawa T (1999) A scatter-free ion acceleration process in the parallel shock. Adv Space Res 24:73-77

Sugiyama T, Fujimoto M, Mukai T (2001) Quick ion injection and acceleration at quasi-parallel shocks. J Geophys Res 106:21657-21673

Terasawa T, Scholer M, Ipavich FM (1985) Anisotropy observation of diffuse ions (greater than $30 \mathrm{keV} / \mathrm{e}$ ) upstream of the earth's bow shock. J Geophys Res 90:249-260

Tidman DA, Krall NA (1971) Shock waves in collisionless plasmas. Wiley-Interscience, New York

Treumann RA (2006) The electron cyclotron maser for astrophysical application. Astron Astrophys Rev 13:229-315

Treumann RA (2008) A note on the theory of transverse diffusion in shock particle acceleration. Theory of super-diffusion for the magnetopause. Geophys Res Lett 24:1727-1730; arXiv: 0811.3938v1 [astro-ph]

Treumann RA, Baumjohann W (1997) Advanced space plasma physics. Imperial College Press, London

Treumann RA, LaBelle J (1992) Band splitting in solar type II radio bursts. Astrophys J 399:L167-L170

Trotignon JG et al (2001) How to determine the thermal electron density and the magnetic field strength from the Cluster/Whisper observations around the Earth. Ann Geophysicae 19:1711-1720

Tsytovich VN (1970) Nonlinear effects in plasmas. Plenum Press, New York

Tu C-Y, Marsch E (1995) MHD structures, waves and turbulence in the solar wind: observations and theories. Space Sci Rev 73:1-210

Veilleux S, Cecil G, Bland-Hawthorn J (2005) Galactic winds. Ann Rev Astron Astrophys 43: 769-826. doi:10.11146/annurev.astro.43.072193.150610

Weibel ES (1959) Spontaneously growing transverse waves in a plasma due to an anisotropic velocity distribution. Phys Rev Lett 2:83-84

Wu CS (1984) A fast Fermi process—energetic electrons accelerated by a nearly perpendicular bow shock. J Geophys Res 89:8857-8862

Wu CC, Kennel CF (1992) Structural relations for time-dependent intermediate shocks. Geophys Res Lett 19:2087-2090

Zahibo N et al (2007) Strongly nonlinear steepening of long interfacial waves. Nonlinear Process Geophys 14:247-256

Zeldovich YB, Raizer YP (1966/1967) Physics of shock waves and high-temperature hydrodynamic phenomena, vols $1 \& 2$. Academic Press, New York

Ziegler HJ, Schindler K (1988) Structure of subcritical perpendicular shock waves. Phys Fluids 31:570-576 